\title{
CONTROL OF STATIONARY CROSS-FLOW MODES \\ IN A MACH 3.5 BOUNDARY LAYER USING PATTERNED PASSIVE AND ACTIVE ROUGHNESS
}

\author{
A Dissertation \\ Submitted to the Graduate School \\ of the University of Notre Dame \\ in Partial Fulfillment of the Requirements \\ for the Degree of
}

Doctor of Philosophy

by

Chan Yong Schuele

Thomas C. Corke, Director

Graduate Program in Aerospace and Mechanical Engineering

Notre Dame, Indiana

December 2011 
(c) Copyright by

Chan Yong Schuele

2011

All Rights Reserved 


\title{
CONTROL OF STATIONARY CROSS-FLOW MODES
}

\section{IN A MACH 3.5 BOUNDARY LAYER USING PATTERNED PASSIVE AND ACTIVE ROUGHNESS}

\author{
Abstract \\ by \\ Chan Yong Schuele
}

Spanwise-periodic roughness designed to excite selected wavelengths of stationary cross-flow modes was investigated in a 3-D boundary layer at Mach 3.5. The test model was a sharp-tipped $14^{\circ}$ right-circular cone. The model and integrated sensor traversing system were placed in the Mach 3.5 Supersonic Low Disturbance Tunnel (SLDT) equipped with a "quiet design" nozzle at the NASA Langley Research Center. The model was oriented at a $4.2^{\circ}$ angle of attack to produce a mean cross-flow velocity component in the boundary layer over the cone. Five removable cone tips have been investigated. One has a smooth surface that is used to document the baseline ("natural") conditions. Two had minute $(20-40 \mu \mathrm{m})$ "dimples" that are equally spaced around the circumference, at a streamwise location that is just upstream of the linear stability neutral growth branch for cross-flow modes. The azimuthal mode numbers of the dimpled tips were selected to either enhance the most amplified wave numbers, or to suppress the growth of the most amplified wave numbers. Two of the cone tips had an array of plasma streamwise vortex generators that were designed to simulate the disturbances produced by the passive patterned roughness. The results indicate that the stationary cross-flow 
Chan Yong Schuele modes were highly receptive to the patterned roughness of both passive and active types. The patterned passive roughness that was designed to suppress the growth of the most amplified modes had an azimuthal wavelength that was $66 \%$ smaller that that of the most amplified stationary cross-flow mode. This had the effect to increase the transition Reynolds number from $25 \%$ to $50 \%$ depending on the measurement technique. The application of the research is on turbulent transition control on swept wings of supersonic aircraft. The plasma-based roughness has the advantage over the passive roughness of being able to be adaptable to different conditions that would occur during a flight mission. 
The dissertation is dedicated to my family. 


\section{CONTENTS}

FIGURES . . . . . . . . . . . . . . . . . . . . . . . vi

ACKNOWLEDGMENTS ................... . . $\mathrm{XV}$

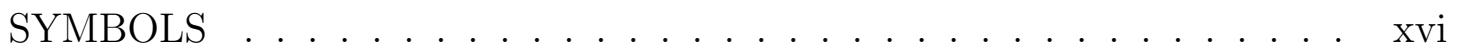

CHAPTER 1: INTRODUCTION . . . . . . . . . . . . . 1

1.1 Motivation .................... 1

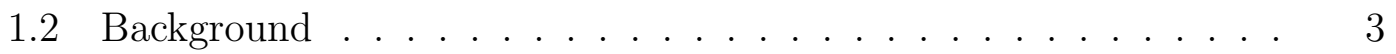

1.2.1 Overview Laminar-Turbulent Transition . . . . . . . . . 3

1.2.2 The Cross-flow Dominated Laminar-Turbulent Transition in Detail ..................... 4

1.2.2.1 Cross-flow Basics . . . . . . . . . . . . . . 4

1.2.2.2 Excitation of Defined Cross-flow Wavenumbers . . . . 6

1.2.2.3 Distributed Roughness Element Cross-flow Experiments in Supersonic Boundary Layers . . . . . . . . . . . 8

1.2.3 Swept Wing vs. Cone . . . . . . . . . . . . . . . 11

1.2.4 Transition Experiments with Cones . . . . . . . . . 11

1.2.5 Choice of Wind Tunnel . . . . . . . . . . . . 15

1.2 .6 DBD Plasma Bumps ................. 15

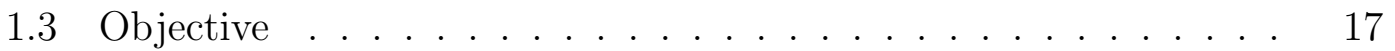

CHAPTER 2: EXPERIMENTAL SETUP . . . . . . . . . . . . 19

2.1 Wind Tunnel ..................... . . . 19

2.2 Cone Model . . . . . . . . . . . . . . . . . . . . . . 22

2.3 Probe Traversing Mechanism . . . . . . . . . . . . . 22

2.3.1 Calibration Procedure and Estimation of Uncertainty for the LVDT Sensor . . . . . . . . . . . . . 26

2.3.2 Offset Estimation Procedure . . . . . . . . . . 27

2.3 .3 Pitot Probe . . . . . . . . . . . . . . . 29

2.4 Data Acquisition . . . . . . . . . . . . . 30 
2.5 Numerical Stability Analysis . . . . . . . . . . . . . . . 30

2.6 Disturbance Generators . . . . . . . . . . . . . . . 31

2.6.1 Passive Mechanical Dimples . . . . . . . . . . . . . 33

2.6.2 Plasma Disturbance Generators . . . . . . . . . . . . 33

2.6.2.1 Individual Circular Plasma Dots . . . . . . . . . . . 35

2.6.2.2 Serrated Edge / Comb actuator . . . . . . . . . . . . 37

2.6.2.3 Vapor Deposition . . . . . . . . . . . . . . . . 37

2.6.2.4 Kapton Film Actuator . . . . . . . . . . . . . . . . 39

2.6.2.5 Dielectric Cone Tip Actuator . . . . . . . . . . . 42

2.6.3 Surface Roughness Characterization of the Cone Tips . . . 47

CHAPTER 3: MICRO DBD PLASMA ROUGHNESS ARRAY EVALUA-

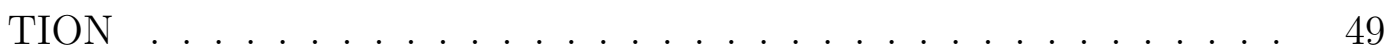

3.1 Circular Plasma Dots . . . . . . . . . . . . . . . . . . . . 49

3.2 Serrated edge actuator . . . . . . . . . . . . . . 553

CHAPTER 4: RESULTS WITH PASSIVE DISTRIBUTED ROUGHNESS 63

4.1 Surface Flow Visualization . . . . . . . . . . . . . 63

4.2 Pitot Tube Surveys . . . . . . . . . . . . . . . . . . . . 69

4.2.1 Receptivity to Forcing Wavenumber . . . . . . . . . . . . 69

4.2 .2 Cross Flow Vortex Path . . . . . . . . . . . . . . . 75

4.2.3 Detailed Wavenumber Analysis . . . . . . . . . . . 79

4.2 .4 Repeatability ... . . . . . . . . . . . . . 81

4.3 Transition Front Detection . . . . . . . . . . . . . 83

CHAPTER 5: RESULTS WITH DBD PLASMA ROUGHNESS . . . . . 100

5.1 Pitot Tube Surveys: Receptivity to Excitation Wavenumber . . . 100

5.2 Repeatability . . . . . . . . . . . . . . . . . . . 108

5.3 Effect of Initial Amplitudes _. . . . . . . . . . . . . . 109

CHAPTER 6: DISCUSSIONS AND CONCLUSIONS . . . . . . . . . 112

6.1 Patterned Roughness . . . . . . . . . . . . . . . . 113

6.2 Receptivity to the Patterned Roughness . . . . . . . . . . . 114

6.2.1 Flow Visualization . . . . . . . . . . . . . . . . . . 114

6.2 .2 Pitot Probe Measurements . . . . . . . . . . . . . . . 114

6.3 Laminar-Turbulent Transition Effect . . . . . . . . . . . . . 117

CHAPTER 7: RECOMMENDATIONS FOR FUTURE WORK . . . . . 120

7.1 Experimental Work . . . . . . . . . . . . . . . 120

7.2 Numerical Work . . . . . . . . . . . . . . . . . . . . . . 123 
APPENDIX A: UNCERTAINTY CALCULATIONS . . . . . . . . . . 124

A.1 LVDT Wall Normal Motion . . . . . . . . . . . . . . . . 124

APPENDIX B: PRESSURE TRACE WAVENUMBER ANALYSIS . . . . 126

APPENDIX C: FAST RESPONSE PITOT PROBE AND HOT WIRE

MEASUREMENTS . . . . . . . . . . . . . . . . . . . . . 138

C.1 Pitot Probe Analysis . . . . . . . . . . . . . . . . . . . 138

C.1.1 Sinclair and Robins . . . . . . . . . . . . . . . . 138

C.1.2 Helmhotz Resonator . . . . . . . . . . . . . . . . . . . . 142

C.1.3 Damped Wave Equation . . . . . . . . . . . . . . . 146

C.1.4 Pitot Probe Conclusions . . . . . . . . . . . . . . . . 149

C.2 Hot Wire Measurements . . . . . . . . . . . . . . . . 151

APPENDIX D: DBD THRUST MEASUREMENTS . . . . . . . . . 152

D.1 Experimental DBD Plasma Body Force Study in Sub-Atmospheric

Pressure . . . . . . . . . . . . . . . . 152

D.1.1 Experimental Setup . . . . . . . . . . . . . . 153

D.1.2 Actuator Measurements . . . . . . . . . . . . 154

BIBLIOGRAPHY . . . . . . . . . . . . . . . . . . 162 


\section{FIGURES}

1.1 Schematic of cross-flow, adapted from White et al. [60]. . . . . .

1.2 Streamwise velocity contours of regularly forced cross-flow vortices with $12 \mathrm{~mm}$ in low speed flow are shown on the left hand side. The right hand side shows the equivalent location with natural roughness. Picture taken from Saric and Reed [46]. . . . . . . . .

1.3 Naphthalene flow visualization on a $45^{\circ}$ sweep wing at a chord Reynolds number of $R e_{c}=2 \times 10^{6}$. Left hand side shows natural roughness case and left hand side shows subcritical forcing with $8 \mathrm{~mm}$ spacing and $6 \mu \mathrm{m}$ height bumps. Flow is from left to right. Well visible is the delayed transition to turbulence and a regular $8 \mathrm{~mm}$ pattern of cross-flow vortices on the right hand side picture [47]. . . . . . . . . . . . . . . . . . . . . . . . .

1.4 Infrared thermographic images of a $30^{\circ}$ swept wing at $M=1.85$ under a F15 jet comparing subcritical forcing to natural roughness as found in [48]. The flow is moving from right to left. Dark regions show lower temperature than brighter regions. . . . . . . . .

1.5 Cross-flow Reynolds number contours on the $7^{\circ}$ half angle cone at $4.2^{\circ}$ angle of attack. The black lines represent inviscid surface streamlines. Picture is adapted from Balakumar [3]. . . . . . .

1.6 Boundary layer velocity profiles at constant axial location $20.3 \mathrm{~cm}$ (8 in) downstream from the cone tip at different azimuthal angles from $0^{\circ}$ to $180^{\circ}$. Picture is taken from Balakumar [3]. . . . . . .

2.1 NASA Langley SLDT wind tunnel. View on test section and the bleed system (2D rectangular nozzle shown). . . . . . . . . . . 20 20

2.2 Cross section of the NASA Langley Mach 3.5 SLDT axisymmetric nozzle. The picture is showing the quiet test core and its constraints due to radiated noise. . . . . . . . . . . . . . .

2.3 Schlieren image of the cone model inside the quiet core of the supersonic nozzle at Mach 3.5. Bleed valves are open and Görtler vortices are present. The quiet core is free of shocks. . . . . . . . 21 
2.4 Cross section of the $7^{\circ}$ half angle cone with dimensions including the mounted removable cone tip. The drawing does not include the axial mounting sting. Units are in inches. . . . . . . . . . . 23

2.5 Traverse seen from the side. . . . . . . . . . . . . . 23

2.6 The tip of the traverse arm: Removable wedge with Pitot tube and LVDT sensor for positioning. . . . . . . . . . . . . . 25

2.7 Schematic of the optical rotary encoder RIK 2C 64/2048 L1-W3 from Numerik Jena. . . . . . . . . . . . . . . . . . . 25

2.8 LVDT displacement sensor for the wall normal motion of the wedge and Pitot tube. . . . . . . . . . . . . . 26

2.9 Calibration curve for the LVDT sensor. . . . . . . . . . . . 28

2.10 Rays of constant azimuthal angle on the cone. . . . . . . . . . 31

2.11 Growth rates for axial rays at constant azimuthal angle $\theta$ obtained from Balakumar's linear stability calculations [3] at $R e_{\infty}=9.8 \times$

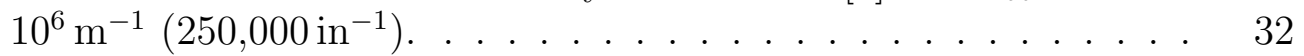

2.12 Photographs of the $m=45$ cone tip, and magnified views of the $m=45$ and $m=68$ dimples, including the topography across the dimples. . . . . . . . . . . . . . . . 34

2.13 Schematic drawing of a single plasma dot actuator arrangement including the induced flow direction (not to scale). . . . . . . .

2.14 Left hand side: Schematic of the streamwise vorticity generator as used by Saric and Corke in 2001. Right hand side: Drawing of the comb actuator geometry as used in the current supersonic experiments. $\lambda$ is the wavelength of the desired stationary mode. $h=\lambda$ and $w=\lambda / 2 \ldots \ldots \ldots$

2.15 Enlarged image of the exposed electrode after lift off. Each dot has a diameter of $76 \mu \mathrm{m}(0.003 \mathrm{in})$ and is spaced approximately $152 \mu \mathrm{m}$ (0.006 in) apart. The substrate is a $12.7 \mu \mathrm{m}(0.5$ mil) thick Kapton film. ...................... 41

$2.1612 .7 \mu \mathrm{m}$ (0.5 mil) thick Kapton actuator glued to a Torlon carrier cone. Right hand side picture shows the operating plasma dots at $10.2 \mathrm{kPa}(3 \mathrm{inHg})$ ambient pressure with $2.2 \mathrm{kVpp}$ and $3 \mathrm{kHz}$ sinwave. ......................... 41

2.17 Drawing of the Macor/aluminum cone tip showing the cross section. 44

$2.18127 \mu \mathrm{m}$ (5 mil) diameter Plasma Dots with $m=45$ spacing on a Macor dielectric cone tip . . . . . . . . . . . . 46 46 
2.19 (a) shows the $m=45$ serrated edge design on the Macor cone tip. (b) is the magnified view of a $m=68$ plasma roughness tip with a Keyence Model VHX-600 3-D Imaging Microscope, and (c) is a high resolution optical image of four single "fingers" of image (b). Photograph (b) and (c) credit: Stephen Wilkinson (NASA LaRC)

2.20 Time averaged images show the plasma pattern that forms on the Macor cone tip. $5 \mathrm{kVpp}, 10.2 \mathrm{kPa}(3 \mathrm{inHg})$ ambient pressure and $5 \mathrm{kHz}$ sine waves are applied. Concentrations of plasma are formed around the tips of the "fingers" of the serrated edge. Additionally a two dimensional plasma sheet can be seen. . . . . . . . . . . .

48

3.1 Modeling of the circular plasma actuator in Comsol. $\mathrm{D}=$ dielectric thickness, $\mathrm{r}=$ radius of the circular dot and $\mathrm{t}=$ thickness of the electrode. $\frac{t}{D}$ stays constant and is $4 \times 10^{-4}$. Dielectric Constant $\mathrm{k}=6$ for Macor. . . . . . . . . . . . . . . . . . . .

3.2 Surfaces of the normalized potential $U / U_{0}$ for $\mathrm{r} / \mathrm{D}=0.56, \mathrm{r} / \mathrm{D}=1.56$ and the standard straight edge arrangement $(r \rightarrow \infty)$ for mesh A.

3.3 Comparison of the electrical field $E^{*}$ with the unit $\frac{V / V}{i n / i n}$ along the dielectric surface for mesh A. . . . . . . . . . . . . . . .

3.4 Schematic drawing of the $8 \mathrm{x}$ magnified $m=45$ equivalent serrated edge actuator with plasma-on at atmospheric pressure, $24 \mathrm{kVpp}$ and $5 \mathrm{kHz}$ sine waves. The right hand side shows a photograph of the $m=45$ Macor cone tip, $10.2 \mathrm{kPa}$ (3inHg) ambient pressure, $5 \mathrm{kVpp}, 5 \mathrm{kHz}$ sine waves . . . . . . . . . . . .

3.5 Drawing of the PIV light sheet and camera arrangement relative to the exposed serrated edge electrode . . . . . . . . . . . . .

3.6 Velocity magnitude of the first plane at $\mathrm{x}=0 \mathrm{~cm}$ of $\mathrm{v}$ and $\mathrm{w}$ including streamlines. . . . . . . . . . . . . . .

3.7 Velocity magnitude of $\mathrm{v}$ and $\mathrm{w}$ in the $\mathrm{z}$-plane at $\mathrm{z}=0.85 \mathrm{~cm}$. This plane was interpolated from a series of $26 \mathrm{x}$-planes (see Figure 3.6 ) starting at $\mathrm{x}=0 \mathrm{~cm}$ and ending at $\mathrm{x}=0.35 \mathrm{~cm} \ldots \ldots$

3.8 Drawing of the PIV light sheet and camera arrangement relative to the exposed serrated edge electrode . . . . . . . . . .

$3.9 \mathrm{z}$-Vorticity of the serrated edge arrangement. The z-plane is positioned about $0.2 \mathrm{~cm}$ downstream of the actuator edge. . . . . . 
4.1 Surface flow visualization at different unit Reynolds numbers and flow conditions at Mach 3.5. $\mathrm{P}_{0}=172.4 \mathrm{kPa}(25 \mathrm{psi}) \rightarrow R e=9.84 \times$ $10^{6} \mathrm{~m}^{-1}, \mathrm{P}_{0}=241.3 \mathrm{kPa}(35 \mathrm{psi}) \rightarrow R e=1.37 \times 10^{7} \mathrm{~m}^{-1}, \mathrm{P}_{0}=289.6 \mathrm{kPa}$ $(42 \mathrm{psi}) \rightarrow R e=1.65 \times 10^{7} \mathrm{~m}^{-1}$ and $\mathrm{P}_{0}=344.7 \mathrm{kPa}(50 \mathrm{psi}) \rightarrow R e=1.96 \times$ $10^{7} \mathrm{~m}^{-1}$....................... 65

4.2 Schematic of the unwrapping of the conical surface onto the observer plane of the camera. . . . . . . . . . . . 66 66

4.3 Example showing the original and the projected image used for further analysis. . . . . . . . . . . . . . . 67 67

4.4 Surface flow visualization and wavenumber spectra for the smooth baseline case, the critical wavenumber baseline case, and the subcritical case at different axial locations. . . . . . . . . . 68 68

4.5 Pitot probe azimuthal scans (a), amplitude spectra (b), and wavelets (c) at $38 \mu \mathrm{m}(0.0015 \mathrm{in})$ above the surface and $\mathrm{x}=28.45 \mathrm{~cm}$ (11.2 in), $m=0$ cone tip, $\mathrm{P}_{0}=172.4 \mathrm{kPa}(25 \mathrm{psi}) \ldots \ldots$. . . . . . . 70

4.6 Pitot Probe azimuthal scans (a), amplitude spectra (b), and wavelets (c) at $38 \mu \mathrm{m}(0.0015 \mathrm{in})$ above the surface and $\mathrm{x}=28.45 \mathrm{~cm}$ (11.2 in)

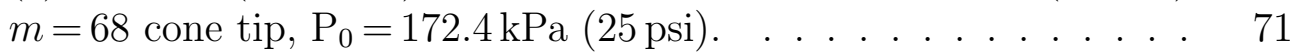

4.7 Pitot probe azimuthal scans (a), amplitude spectra (b), and wavelets (c) at $38 \mu \mathrm{m}(0.0015 \mathrm{in})$ above the surface and $\mathrm{x}=28.45 \mathrm{~cm}(11.2 \mathrm{in})$ with the $m=45$ cone tip, $\mathrm{P}_{0}=172.4 \mathrm{kPa}(25 \mathrm{psi}) . \ldots 72$

4.8 Pitot probe azimuthal scans (a), amplitude spectra (b), and wavelets (c) at $38 \mu \mathrm{m}(0.0015 \mathrm{in})$ above the surface and $\mathrm{x}=25.9 \mathrm{~cm}(10.2 \mathrm{in})$, $m=68$ cone tip, $\mathrm{P}_{0}=172.4 \mathrm{kPa}(25 \mathrm{psi}) . \ldots$. . . . . . .

4.9 Pitot probe azimuthal scans (a), amplitude spectra (b), and wavelets (c) at $38 \mu \mathrm{m}(0.0015 \mathrm{in})$ above the surface and $\mathrm{x}=25.9 \mathrm{~cm}(10.2 \mathrm{in})$, $m=45$ cone tip, $\mathrm{P}_{0}=172.4 \mathrm{kPa}(25 \mathrm{psi}) \ldots \ldots \ldots$

4.10 Pitot tube scans at different axial and azimuthal locations at $38 \mu \mathrm{m}$

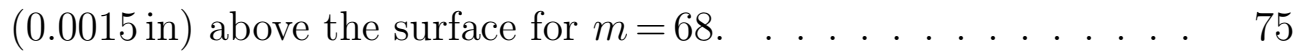

4.11 Cross-flow vortex paths with the $m=68$ roughness tip in comparison with the surface streamlines of the baseline flow and vortex paths of Balakumar's DNS calculations [3] with a $m=40$ distributed disturbance at $\mathrm{x}=1.27 \mathrm{~cm}(0.5 \mathrm{in})$. (a) shows the $\mathrm{x}-\theta$ plane and (b) corresponds to the projection on the observer $\mathrm{x}-\mathrm{y}$ plane. .

$4.12 m=68$ roughness tip, azimuthal scan at $\mathrm{x}=12.7 \mathrm{~cm}(5 \mathrm{in}), 0.05 \mathrm{~mm}$ (0.002 in) Pitot probe height. Wavenumber spectrum (center) and Wavelet analysis (bottom). $\mathrm{P}_{0}=172.4 \mathrm{kPa}(25 \mathrm{psi}) \ldots . .$. . . 
4.13 Comparison of the dominant wavenumbers with the $m=68$ dimple tip at $172.4 \mathrm{kPa}(25 \mathrm{psia})$ for different Reynolds numbers at $0.05 \mathrm{~mm}$ (0.002 in) height above surface. (a) and (b) correspond to the zones wind- and leeward of the parting vortex trace. . . . . . . . .

4.14 Repeated measurements of azimuthal pressure distributions at constant axial position. The left hand side shows the non-repeatability for the smooth tip and a well repeatable pattern for the $m=68$ cone tip. $\mathrm{P}_{0}=172.4 \mathrm{kPa}(25 \mathrm{psi}) \ldots \ldots \ldots \ldots$

4.15 Wavenumber analysis of the repeated Pitot probe measurements with the $m=0$ and $m=68$ cone tip at $\mathrm{x}=25.4 \mathrm{~cm}(10 \mathrm{in})$. The total pressure is $\mathrm{P}_{0}=172.4 \mathrm{kPa}(25 \mathrm{psi}) \ldots . . . . . .$.

4.16 Pitot probe measurements at constant azimuthal angle $\left(\theta=120^{\circ}\right)$ and three different free stream unit Reynolds numbers: $R e_{25 p s i}=$ $0.25 \times 10^{6} \mathrm{in}^{-1}, R e_{35 p s i}=0.35 \times 10^{6} \mathrm{in}^{-1}$ and $R e_{45 p s i}=0.45 \times 10^{6} \mathrm{in}^{-1} .85$

4.17 Pitot probe measurements for $m=45$ (top) and $m=68$ (bottom) patterned roughness. $\mathrm{P}_{0}=310.3 \mathrm{kPa}(45 \mathrm{psi}) \ldots \ldots$. . . . .

4.18 Preston probe measurements for $m=45$ (top) and $m=68$ (bottom) forcing at $\mathrm{Ma}=3.5$ and a freestream unit Reynolds number of $1.77 \times$ $10^{7} \mathrm{~m}^{-1}\left(4.5 \times 10^{5} \mathrm{in}^{-1}\right)$ and $\mathrm{P}_{0}=310.3 \mathrm{kPa}(45 \mathrm{psi})$. The heights correspond to the normalized total pressure at the probe tip. . . .

4.19 Axial development of the RMS of the pressure fluctuations in the azimuthal direction that is associated with the cross-flow vortices.

4.20 Preston probe method showing sharp rise in measured total pressure with increasing free stream Reynolds number at the location of transition onset. Figure was taken from [28]. . . . . . . . . .

4.21 Straightened Preston probe scans showing the normalized total pressures and paths of the analyzed pressure traces for the detection of laminar-turbulent transition. $\mathrm{P}_{0}=310.3 \mathrm{kPa}(45 \mathrm{psi}) \ldots . .$.

$4.22 m=45$ pressure traces parallel to the cross-flow vortex structure centered between the two visible pressure maxima. $0.5^{\circ}$ azimuthal spacing between each trace, covering approximately $6^{\circ}$ from $105^{\circ}$ to $111^{\circ}$ in azimuthal direction. The dashed red line is a linear curve fitted to the slope of the pressure signal. The intersection with $p_{t} / p_{0}=0.022$ is defined as laminar turbulent transition onset. . .

$4.23 m=68$ normalized pressure traces parallel to the cross-flow vortex structure. There are two low pressure regions (a) and (b) for which the transition Reynolds number $R e_{t r}$ is estimated. The angles correspond to the initial angle where the measurement of the traces was started. 
4.24 Shown are the laminar-turbulent transition locations for the $m=45$ and $m=68$ distributed roughness tips including error bars. The black circular dots (a) and (b) correspond to the traces in Figure 4.21 with $m=68$ forcing. The blue dot corresponds to the location identified in Figure 4.22 with $m=45$ forcing. . . . . . . . . . . .

4.25 The top figure is adapted from King [28] and shows the qualitative shape of the transition front. Added is the shape of the transition front based on Balakumar's linear stability analysis [3]. The bottom figure shows the Preston probe and coherent structure based turbulent transition locations including curves of constant turbulent transition $\mathrm{N}$-factors. These correspond to $N \approx 6$ for $m=45$ and $N \approx 9$ for $m=68$. . . . . . . . . . . . . . . . 96

4.26 Wavenumber amplitude spectrum of the normalized total pressures from Figure 4.17 with the $m=45$ cone tip . . . . . . . . . . . . .

4.27 Wavenumber amplitude spectrum of the normalized total pressures from Figure 4.17 with the $m=68$ cone tip . . . . . . . . .

5.1 Pitot probe traces at $0.027 \mathrm{~mm}$ (0.0011 in) height above the surface, $\mathrm{P}_{0}=172.4 \mathrm{kPa}(25 \mathrm{psia}), T_{0}=311^{\circ} \mathrm{K}\left(100^{\circ} \mathrm{F}\right) . m=45 \mathrm{DBD}$ plasma cone tip. Vortex traces are outlined with dashed lines. The blue area corresponds to the zone where forcing at $1.27 \mathrm{~cm}(0.5 \mathrm{in})$ has no influence (from Figure 4.11.) . . . . . . . . . . . . . 101

5.2 Pitot probe traces at $0.04 \mathrm{~mm}$ (0.0016 in) height above the surface, $\mathrm{P}_{0}=172.4 \mathrm{kPa}\left(25\right.$ psia), $T_{0}=100^{\circ} \mathrm{F} . m=68 \mathrm{DBD}$ plasma cone tip. Vortex traces are outlined with dashed lines. The blue area corresponds to the zone where forcing at $1.27 \mathrm{~cm}(0.5 \mathrm{in})$ has no influence (from figure 4.11.) . . . . . . . . . . . . . 103

5.3 Azimuthal Pitot probe scans with the $m=45$ plasma cone tip at $\mathrm{x}=15.2 \mathrm{~cm}(6 \mathrm{in})$ and $R e_{x}=1.5 \times 10^{6}$. The FFT is applied to the white area of the Pitot probe data series. $\mathrm{P}_{0}=172.4 \mathrm{kPa}(25 \mathrm{psi})$.

5.4 Azimuthal Pitot probe scans with the $m=45$ plasma cone tip at $\mathrm{x}=17.8 \mathrm{~cm}(7 \mathrm{in})$ and $R e_{x}=1.75 \times 10^{6}$. The FFT is applied to the white area of the Pitot probe data series. $\mathrm{P}_{0}=172.4 \mathrm{kPa}(25 \mathrm{psi})$. 105

5.5 Azimuthal Pitot probe scans with the $m=45$ plasma cone tip at $\mathrm{x}=20.3 \mathrm{~cm}(8 \mathrm{in})$ and $R e_{x}=2 \times 10^{6}$. The FFT is applied to the white area of the Pitot probe data series. $\mathrm{P}_{0}=172.4 \mathrm{kPa}(25 \mathrm{psi})$. 106

5.6 Azimuthal Pitot probe scans with the $m=68$ plasma cone tip at $\mathrm{x}=15.2 \mathrm{~cm}(6 \mathrm{in})$ and $R e_{x}=1.5 \times 10^{6}$. The FFT is applied to the white area of the Pitot probe data series. $\mathrm{P}_{0}=172.4 \mathrm{kPa}(25 \mathrm{psi})$. 106 
5.7 Azimuthal Pitot probe scans with the $m=68$ plasma cone tip at $\mathrm{x}=17.8 \mathrm{~cm}(7 \mathrm{in})$ and $R e_{x}=1.75 \times 10^{6}$. The FFT is applied to the white area of the Pitot probe data series. $\mathrm{P}_{0}=172.4 \mathrm{kPa}(25 \mathrm{psi})$. 107

5.8 Azimuthal Pitot probe scans with the $m=68$ plasma cone tip at $\mathrm{x}=20.3 \mathrm{~cm}(8 \mathrm{in})$ and $R e_{x}=2 \times 10^{6}$. The FFT is applied to the white area of the Pitot probe data series. $\mathrm{P}_{0}=172.4 \mathrm{kPa}(25 \mathrm{psi})$. 108

5.9 Repeated azimuthal pressure scans with plasma on before and after a wind tunnel restart. $m=68, \mathrm{P}_{0}=172.4 \mathrm{kPa}(25 \mathrm{psi}), T_{0}=311 \mathrm{~K}$

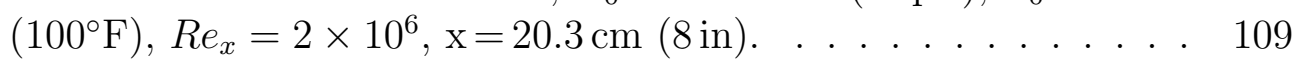

5.10 Repeated azimuthal pressure scans with AC driving frequencies from $1 \mathrm{kHz}$ to $6 \mathrm{kHz}$ and different voltages. $m=68, R e_{x}=2 \times 10^{6}$, $\mathrm{x}=20.3 \mathrm{~cm}(8 \mathrm{in}), \mathrm{P}_{0}=172.4 \mathrm{kPa}(25 \mathrm{psi}), T_{0}=311 \mathrm{~K}\left(100^{\circ} \mathrm{F}\right) \ldots$

A.1 Drawing of the Pitot tube, Balsa wood calibration piece and mounting wedge.

B.1 FFTs of a representative sinusoidal test signal with varying sample lengths.

B.2 The original data series from Figure B.1 was mirrored two times and then analyzed with a FFT.

B.3 Each mirrored data series was phase shifted in order to remove step changes at the end of the original signal. . . . . . . . . . . . 131

B.4 $m=45, R e_{x}=1.5 \times 10^{6}, \mathrm{x}=12.7 \mathrm{~cm}$ (6 in). Summary of the original data series (a), the high pass filtered signal (b), the extended data series (b), and the respective FFTs. The blue regions mark the areas windward of the parting vortex trace. . . . . . . . . . . 132

B.5 $m=45, R e_{x}=1.75 \times 10^{6}, \mathrm{x}=17.8 \mathrm{~cm}(7 \mathrm{in}), \mathrm{P}_{0}=172.4 \mathrm{kPa}(25 \mathrm{psi})$. Summary of the original data series (a), the high pass filtered signal (b), the extended data series (b), and the respective FFTs. The blue regions mark the areas windward of the parting vortex trace.

B.6 $m=45, R e_{x}=2 \times 10^{6}, \mathrm{x}=20.3 \mathrm{~cm}(8 \mathrm{in}), \mathrm{P}_{0}=172.4 \mathrm{kPa}(25 \mathrm{psi})$. Summary of the original data series (a), the high pass filtered signal (b), the extended data series (b), and the respective FFTs. The blue regions mark the areas windward of the parting vortex trace. 134

B.7 $m=68, R e_{x}=1.5 \times 10^{6}, \mathrm{x}=12.7 \mathrm{~cm}(6 \mathrm{in}), \mathrm{P}_{0}=172.4 \mathrm{kPa}(25 \mathrm{psi})$. Summary of the original data series (a), the high pass filtered signal (b), the extended data series (b), and the respective FFTs. The blue regions mark the areas windward of the parting vortex trace. 
B.8 $m=68, R e_{x}=1.75 \times 10^{6}, \mathrm{x}=17.8 \mathrm{~cm}(7 \mathrm{in}), \mathrm{P}_{0}=172.4 \mathrm{kPa}(25 \mathrm{psi})$. Summary of the original data series (a), the high pass filtered signal (b), the extended data series (b), and the respective FFTs. The blue regions mark the areas windward of the parting vortex trace.

B.9 $m=68, R e_{x}=2 \times 10^{6}, \mathrm{x}=20.3 \mathrm{~cm}(8 \mathrm{in}), \mathrm{P}_{0}=172.4 \mathrm{kPa}(25 \mathrm{psi})$. Summary of the original data series (a), the high pass filtered signal (b), the extended data series (b), and the respective FFTs. The blue regions mark the areas windward of the parting vortex trace.

C.1 Knudsen number for different total pressures and Pitot probe diameters .................. 140

C.2 Bandwidth of the Pitot tube in dependence of the length to diameter ratio and for different ambient total pressures. Data is corrected with Knudsen number as shown in equation C.9. Each line shows the bandwidth for different ambient pressures in $10000 \mathrm{kPa}$ steps . 143

C.3 Transfer function for $0.1 \mathrm{~mm}$ and $0.25 \mathrm{~mm}$ diameter Pitot tubes at different Knudsen numbers for $T_{0}=300 \mathrm{~K}$. . . . . . . . . . . . 145

C.4 Displacement of an infinitesimally thin disc of fluid inside the Pitot tube.

D.1 Teflon plasma actuator on the shielded force balance inside the vacuum vessel. . . . . . . . . . . . . . . . . . 153

D.2 Comparison of thrust measurements inside and outside the vacuum vessel. . . . . . . . . . . . . . . . 154

D.3 Influence of different ambient pressures on the voltage vs. thrust curves. $2 \mathrm{kHz}$ ramps. . . . . . . . . . . . . . . . 155

D.4 Influence of different ambient pressures on the voltage vs. thrust curves. $2 \mathrm{kHz}$ ramps. . . . . . . . . . . . . . . . . . 156

D.5 Maximum thrust values in dependence of ambient pressure for different AC driving frequencies. . . . . . . . . . . . . 157

D.6 Dependence of thrust at constant applied voltage on ambient pressure.157

D.7 Necessary applied voltage to maintain constant body force for different ambient pressures. . . . . . . . . . . . . 158

D.8 Necessary applied voltage to maintain constant body force for different ambient pressures. . . . . . . . . . . . . . . 159

D.9 Thrust measurements for a $1 / 4$ in thick Teflon dielectric with 1 in and 2 in wide covered electrodes at different ambient pressures. . . 160 
D.10 Thrust measurements for a $1 / 8$ in thick Macor dielectric with 1 in and 2 in wide covered electrodes at constant pressures and different AC driving frequencies. . . . . . . . . . . . . . . 161 


\section{ACKNOWLEDGMENTS}

I would like to thank my advisor, Dr. Thomas Corke, who made it possible for me to work on this very interesting research project, that was supported under the NASA NRA/Research Opportunities in Aeronautics Program Cooperative Agreement NNX08AB22A. I am very grateful for his advice and guidance during the years of my $\mathrm{PhD}$ work. My special thanks goes to Dr. Eric Matlis at Notre Dame and Stephen Wilkinson at NASA LaRC who supported this work with their invaluable experience and advice. I would also like to thank Hugh Pinkston, Rhonda, Lewis Owens, and George Beeler at NASA for their support of our experiments at the NASA Langley Research Center. Thank you also to Dr. Ponnampalam Balakumar at NASA LaRC who did the numerical stability analysis of our experiment. Mike Swadener at the Hessert machine shop and Leon Hluchota at the AME Fitzpatrick machine shop supported me with their valuable help in manufacturing my cone tips. I would also like to thank Dr. Patrick Fay and Dave Kopp at the EE Department at Notre Dame for their support in making the plasma cone tips. Without them, learning the necessary techniques of vapor deposition and photo-lithography would have been nearly impossible. 


\section{SYMBOLS}

$A C$ Alternating current
$D$ Dielectric thickness

$D B D \quad$ Dielectric Barrier Discharge

FFT Fast Fourier Transform

$M \quad$ Mach number

$M_{\infty} \quad$ Freestream unit Mach number

$P_{0} \quad$ Free Stream Total Pressure

$P_{t} \quad$ Measured Pitot tube total pressure

Re Reynolds number or unit Reynolds number

$R e_{c}$ Reynolds numbers based on the chord length

TS Tollmien-Schlichting

$m$ Wave number based on $1 / 360^{\circ}$

$k V p p \quad$ Kilovolt peak to peak

mil 1/1000 of an inch

$r$ Radius of the circular DBD actuator

rms Root mean square

$t \quad$ Electrode thickness

$u \quad$ Velocity in $\mathrm{x}$-direction

$x \quad$ Axial coordinate $[\mathrm{cm}]$ 
$\alpha$ Angle of attack

$\nabla$ Gradient

$\theta$ Cone half angle or azimuthal angle

$\Delta x \quad$ Quiet core length 


\section{CHAPTER 1}

\section{INTRODUCTION}

\subsection{Motivation}

The development of commercial supersonic aircraft has been pursued for several decades. After the start of the Concorde program in 1969 and its end in 2003, no other newly developed commercial supersonic airplane became operational although planes like the Tu144 and Boeing's B2707 were developed [22]. For future supersonic aircraft, there are multiple challenges that still need to be overcome. As summarized by Coen et al. [11], these include improving supersonic cruise efficiency, and the need for light weight materials with better durability at high temperatures. Besides purely increasing the efficiency, environmental aspects also have to be considered. These include airport noise, sonic boom, and high altitude

emissions. In the case of the Concorde, the sonic boom prevented supersonic flight over land.

Different approaches can be taken to improve supersonic flight efficiency as well as to address the acoustic emissions. The sonic boom can be mitigated by modifying the shape of the aircraft's nose ("boom shaping" [19]), or adding a nose spike that generates a series of small shocks instead of one large shock ("quiet spike" [23]). However, methods like this for mitigating the sonic boom increase the aerodynamic drag on the aircraft and thus decrease its flight efficiency. Thus, 
in order to maintain flight efficiency while simultaneously addressing the sonic boom, it requires new approaches for lowering the aerodynamic drag elsewhere on the aircraft. A principle area for drag reduction is on the lifting surfaces, which are the main contributor to the overall supersonic aircraft drag.

As first recognized by Adolf Busemann in 1935 [7], wing sweep minimizes wave drag on an aircraft. High sweep angles generate a mean cross-flow over the wing. The cross-flow undergoes a cross-flow instability that is the principle mechanism leading to laminar-turbulent transition on a swept wing. It is desirable to maintain a laminar boundary layer over an as large an area of the wing as possible because laminar-viscous drag is considerably lower than turbulent drag [2]. Other boundary layer instabilities such as Tollmien-Schlichting waves, attachment line instabilities, and a Görtler instability can be minimized by an appropriate shaping of the wing [43]. However, the cross-flow instability is linked to the mean crossflow which exists for larger wing sweep angles that are used in high-supersonic aircraft, and therefore unavoidable.

An active method to limit the occurrence of cross-flow instabilities is wall suction [5]. This method, however, requires complicated hardware that also requires high maintenance. Passive methods on the other hand such as developed by Saric et al. [49] have proven to work efficiently on swept wings and swept flat plates. As shown in different experiments at subsonic speeds, the application of micron sized distributed roughness elements on swept wings [45] can be utilized to control cross-flow dominated laminar-turbulent transition. Depending on the wave number that is excited, the extent of laminar flow can be increased or reduced. Since this method does not require any complicated modification to the wing, it has the potential to be a practical solution for lowering viscous drag on aircraft that 
is commercially viable. As mentioned by Bushnell [8], reliability and maintainability are the key factors for the commercial application of laminar flow control technology.

\subsection{Background}

\subsubsection{Overview Laminar-Turbulent Transition}

Generally, the transition from laminar to turbulent flow undergoes several stages. It starts with external disturbances such as freestream vorticity, freestream sound, or entropy spots, or boundary effects such as roughness or vibrations that are internalized by a process called receptivity. A few descriptions of the term receptivity are given by Bippes [5]: “... the mechanisms leading to the entrainment of external disturbances into the boundary layer and to the generation of instabilities with initial amplitudes dependent on the external disturbances" or Airiau [1]: "Receptivity describes the birth of a disturbance, whereas sensitivity is a concept of larger breath, describing the modification incurred by the state of a system as a response to parametric variations." and by Kachanov [25]: "Receptivity describes processes by means of which instability waves appear in boundary layers under the action of some external perturbation of various physical sources.".

Disturbances are initially damped until they reach the point of neutral amplification. Growth of the disturbances takes place downstream of the point of the first neutral growth Branch I instability. Depending on the initial amplitudes, either linear theory can be used to describe instability growth, or in case of high amplitude initial disturbances, the whole linear regime can be bypassed and only the nonlinear stage including secondary and tertiary modes will develop before transition to turbulence. If the initial amplitudes are somewhere in between, so 
called transient growth can occur.

Modes of linear amplification in a boundary layer include viscous instabilities like the Tollmien-Schlichting waves, inflectional or inviscid instabilities like crossflow instabilities which will be the main interest of this work, adverse pressure gradient or oblique first-mode waves that occur at supersonic Mach numbers, Second TS or Mack modes for hypersonic flows $\left(M_{\infty}>5\right)$, and Görtler vortices for curved streamlines. The nonlinear stage generally develops over a much shorter region than the linear growth regime.

\subsubsection{The Cross-flow Dominated Laminar-Turbulent Transition in Detail}

\subsubsection{Cross-flow Basics}

Cross-flow instability is a property of three-dimensional boundary layer flows. It appears wherever in-plane curved streamlines at a boundary layer edge occur. A schematic of the cross-flow mean velocity profile in a boundary layer is shown in Figure 1.1. Curved streamlines generate centrifugal forces that are balanced by radial pressure gradients. Since static pressure is assumed to be constant in the wall-normal direction within a boundary layer, the excess pressure due to its radial gradient at the boundary layer edge will generate a mean cross-flow. The no-slip condition at the wall leads to zero cross-flow velocity at the wall. At the same time, the cross-flow component is also zero at the edge of the boundary layer, which leads to an inflectional velocity profile that fulfills the necessary condition for an instability according to Rayleigh [51].

The first stage of cross-flow instability dominated transition starts with the process of receptivity. The free-stream turbulence level determines whether travelling or stationary modes will dominate the transition process. Even though 


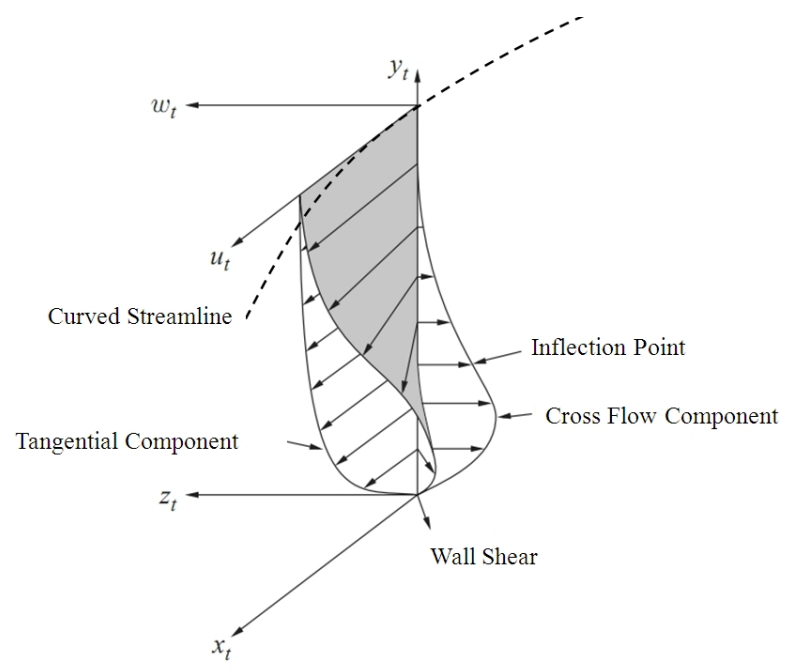

Figure 1.1. Schematic of cross-flow, adapted from White et al. [60].

travelling cross-flow waves are more amplified than stationary cross-flow modes according to linear theory, the stationary modes are sensitive to wall roughness that can provide them with a higher initial amplitude. As a result of their approximate alignment with the inviscid streamlines of the baseline flow, the stationary modes have a strong integrated effect on the boundary layer and can therefore dominate the laminar-turbulent transition process. Deyhle and Bippes [15] estimated background turbulence levels between $0.15 \%$ and $0.3 \%$ were necessary before which travelling modes were the leading mechanism of laminar-turbulent transition.

Stationary cross-flow modes form co-rotating vortices. These grow to an amplitude between $10 \%$ and $30 \%$ [10, 60] at which point nonlinear saturation of the vortices sets in. The growth of the stationary cross-flow vortices leads to the formation of inflected mean profiles. At some point in the nonlinear saturation stage, the inflectional profile leads to an inviscid instability that generates high 
frequency velocity fluctuations that quickly break down to turbulence.

Cross-flow instability experiments were done on subsonic [5, 14, 15, 38] and supersonic [24] swept wings or swept plates, swept cylinders [37], supersonic cones at angles of attack [28], rotating axisymmetric bodies [29], and rotating disks [58]. A comprehensive review has been given by Saric et al. [49] and Reed et al. [39].

\subsubsection{Excitation of Defined Cross-flow Wavenumbers}

Wilkinson and Malik [63] showed in a rotating disk cross-flow experiment that a single isolated roughness element on an otherwise smooth disk surface, fixed the origin of the formation of the emerging stationary cross-flow vortices. Saric et al. [45] exploited this property for his swept wing experiments to excite fixed wavenumber stationary cross-flow modes with arrays of circular micron-sized distributed roughness elements. He showed that stationary cross-flow modes with the forced wavenumber, and its superharmonics, exclusively appeared in the boundary layer. All other wavenumbers of cross-flow modes were completely suppressed. This led to the idea of exciting a weakly amplified stationary cross-flow mode that grows at first and then decays completely. If the wavenumber of this forced mode is larger than the naturally most amplified mode, no super-harmonic modes feed the growth of the most amplified mode, and laminar-turbulent transition is delayed. The initial amplitude of the cross-flow disturbance originating from surface roughness determines the dominant stationary cross-flow wavenumber. Saric called forcing with a wavenumber that is larger than the most amplified "subcritical forcing". Forcing with a wavenumber equal to the naturally most amplified wavenumber is referred to as "critical forcing". U-velocity Pitot probe scans of the stationary cross-flow instability in the boundary layer of a swept wing at 

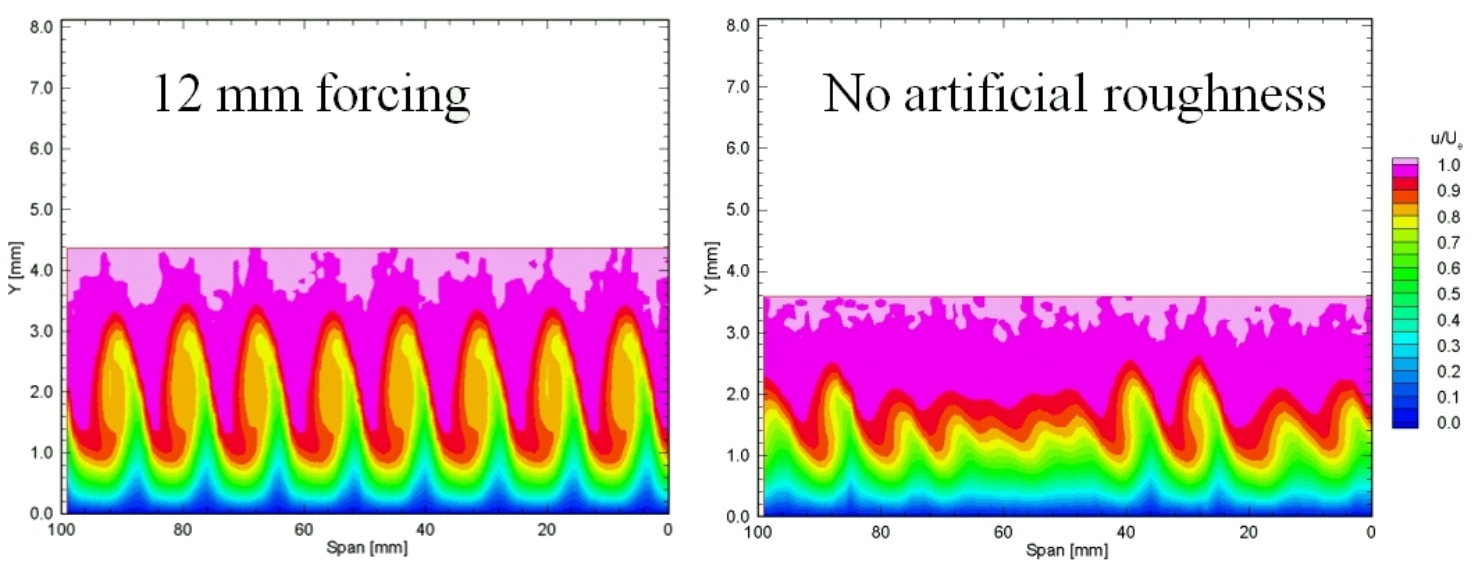

Figure 1.2. Streamwise velocity contours of regularly forced cross-flow vortices with $12 \mathrm{~mm}$ in low speed flow are shown on the left hand side.

The right hand side shows the equivalent location with natural roughness. Picture taken from Saric and Reed [46].

$R e_{c}=3 \times 10^{6}$ without and with patterned roughness forcing that are from Saric et al. [46], are shown in Figure 1.2. The colors indicate mean velocity levels in the boundary layer. The peaks and valleys in the distribution are the result of the mean flow distortion caused by the stationary cross-flow modes. It can be clearly seen that the initially random distribution of stationary pattern without the patterned roughness is replaced by equally spaced stationary pattern when the regular roughness pattern is introduced.

Saric's [42] experiments on swept wings in flight, and low speed wind tunnel tests, showed that the ratio of wavelength to roughness element diameter needs to be at least 0.5 to successfully excite the cross-flow mode. Saric et al. [44] were able to delay laminar-turbulent transition from the $60 \%$ chord location to $80 \%$ chord location with subcritical forcing on a swept wing with a $R e_{c}=2.4 \times 10^{6}$. The transition location moved upstream to $45 \%$ chord length with critical wavenum- 
ber excitation. An example from Saric et al. [47] is shown in Figure 1.3. The laminar-turbulent transition location in this experiment was visualized with the Naphthalene surface flow visualization technique. Naphthalene sublimates faster in regions of higher surface shear stress that will occur in the presence of the stationary cross-flow vortices. These locations will appear as a pattern that is visible in the images. The transition to turbulence is marked by the abrupt end of the white Naphthalene layer due to the higher turbulent shear stress. Contrasting the two images in Figure 1.3, the location of the turbulent transition front has moved significantly downstream as a result of the subcritical forcing.

Saric et al. [45] found that roughness element heights from 6 to $48 \mu \mathrm{m}$ had only a weak effect on the onset of laminar-turbulent transition in Saric's swept wing experiments. This means that effective delay of transition beyond the transition location of exceptionally smooth surfaces can be achieved on surfaces with natural rms-roughness up to $30 \mu \mathrm{m}$ [43], which corresponds to a typical commercial aircraft finish. Additionally, there was no effect of free stream acoustics or 2-D roughness on the formation of stationary cross-flow vortices [38].

\subsubsection{Distributed Roughness Element Cross-flow Experiments in Supersonic Boundary Layers}

Most of the experiments with detailed boundary layer measurements in crossflow dominated laminar-turbulent transition were done at subsonic low Mach number flows. Only two authors, Saric et al. [43, 48] and Semionov et al. [52, 53], investigated the effect of micron sized distributed roughness elements on the location of laminar-turbulent transition under supersonic free stream conditions on swept surfaces. 

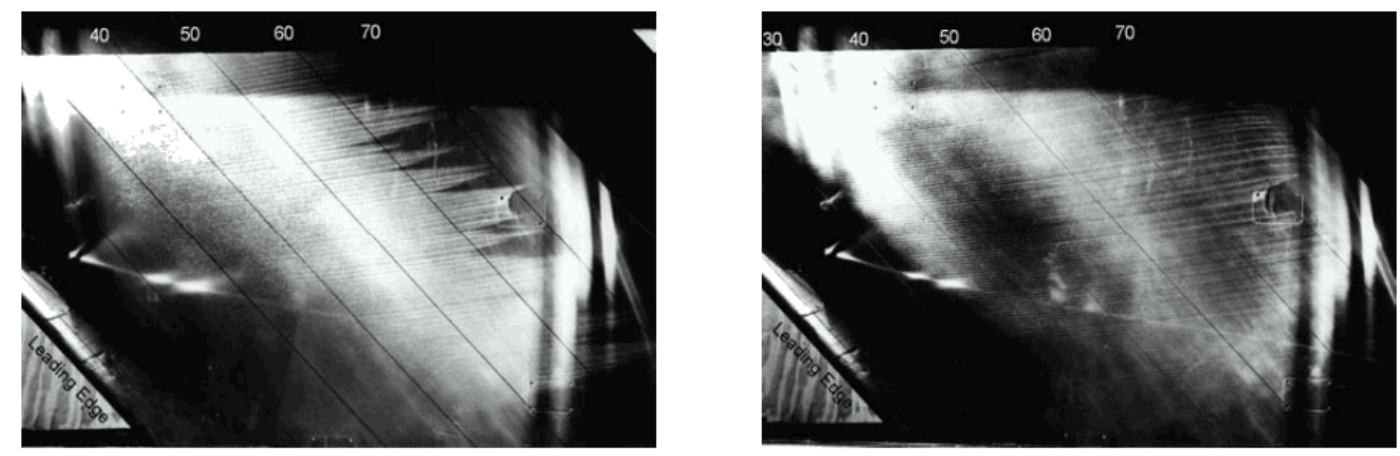

Figure 1.3. Naphthalene flow visualization on a $45^{\circ}$ sweep wing at a chord Reynolds number of $R e_{c}=2 \times 10^{6}$. Left hand side shows natural roughness case and left hand side shows subcritical forcing with $8 \mathrm{~mm}$ spacing and $6 \mu \mathrm{m}$ height bumps. Flow is from left to right. Well visible is the delayed transition to turbulence and a regular $8 \mathrm{~mm}$ pattern of cross-flow vortices on the right hand side picture [47].

Saric and Reed [43] investigated a cooled swept wing in a Mach 2.4 flow. The leading edge sweep angle was large enough to have a subsonic leading edge. They investigated micron-sized bumps and dimples for their roughness array. Infrared thermographic images were used to indicate the onset of turbulent transition by an increase of the wall temperature due to enhanced heat transfer in a turbulent boundary layer. Using a subcritical wavenumber, they could delay transition compared to regions with critical forcing.

In 2004, Saric and Reed [48] performed an experiment on a swept wing with applied subcritically spaced roughness under the fuselage of a F15 jet. Infrared thermography was used to visualize the location of turbulent transition, which showed up as region of enhanced heat transfer. The results indicated that transition delay occurred over a very limited span, towards the tip of the test wing. This is shown in the infrared images from Saric and Reed [48] in Figure 1.4. They mentioned that the limited spanwise effect on the laminar-turbulent transition 


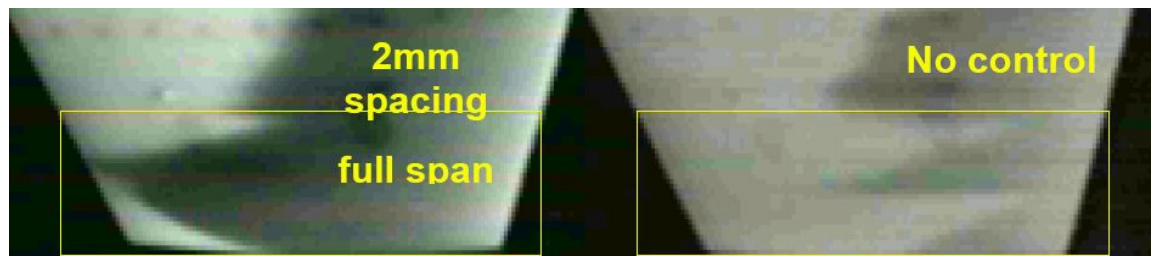

Figure 1.4. Infrared thermographic images of a $30^{\circ}$ swept wing at $M=1.85$ under a F15 jet comparing subcritical forcing to natural roughness as found in [48]. The flow is moving from right to left. Dark regions show lower temperature than brighter regions.

location might have been due to flow interference from the under-body of the aircraft.

Semionov et al. in 2006 [53] and 2008 [52] conducted measurements on a $40^{\circ}$ swept wing in combination with an array of $6 \mu \mathrm{m}$ height distributed roughness in a Mach 2.0 flow. The transition location showed some response to the distributed roughness. However in contrast to the observations of Saric et al. [49], transition seemed to be highly sensitive to the shape of the roughness, and was slightly delayed when the roughness elements were spaced with the most amplified ("critical") wavenumber. The measured mean flow distortion also did not show the excitation wavenumber. In addition, there was no information given about the background disturbance levels in the wind tunnel, which is crucial in estimating the type of instability that might be excited on the swept wing model.

The experiments performed to date on turbulent transition delay with micronsized roughness in supersonic flows have therefore not been completely conclusive. Therefore, additional experiments are needed to demonstrate the ability of subcritical forcing to delay laminar-turbulent transition in supersonic 3-D boundary layers. 


\subsubsection{Swept Wing vs. Cone}

Although the target application is a swept wing, the flow over a sharp cone at an angle of attack has also been the subject of cross-flow experiments. The conical flow at incidence generates a streamline-normal pressure gradient which, if properly chosen, can lead to cross-flow dominated transition. The basic transition mechanism is therefore identical between the swept wing and a cone at an angle of attack.

Cone models have a distinct advantage over finite swept wing models. In order to experimentally establish cross-flow induced transition on a swept wing, either displacement bodies [21] or contoured walls [60] have to be added to eliminate end-effects. In a supersonic flow, additional bodies will cause Mach waves that radiate into the test section. This would lead to free-stream disturbances that could cause premature transition to turbulence. A cone on the other hand, being a body of revolution, has no end effects. This makes it easier to place it within a finite sized test section with a minimum of added disturbance to the free stream flow.

\subsubsection{Transition Experiments with Cones}

Transition experiments on smooth surface cones at an angle of attack in supersonic and hypersonic flows have been performed for the last several decades. The measurements were performed in different wind tunnels with varying background disturbance levels, and in free-flight experiments. Some of the earlier wind tunnel experiments studying the effect of angle of attack, wall cooling and nose bluntness include Stetson and Rushton in 1967 at Mach 5.5 [57] and Stainback in 1969 at Mach 8 [55]. Other wind tunnel experiments investigating the effect 
of angle of attack on the transition location were done by DiCristina [16] in 1970 at Mach 10, and Mateer [33] in 1972 at Mach 7.4. In 1972, Beckwith et al. [4] reviewed and compared the existing transition measurements of cones at angle of attack at NASA Langley, showing the necessity for well controlled freestream disturbance levels for comparability with different facilities, and applicability to results at free flight conditions. Another study of the effect of nose Bluntness and angle of attack in Hypersonic wind tunnels was presented by Stetson in 1980 [56]. Free flight experiments with cones at angles of attack under adiabatic conditions were performed by Fisher et al. in 1982 [18] at low supersonic speeds from $\mathrm{M}=0.5$ to 2.0. Special low disturbance wind tunnels were used by King in 1992 [28] in the NASA Langley Mach 3.5 SLDT, and by Wolf and Laub in 1997 in the NASA Ames Mach 1.6 LFSWT [65] to obtain transition data with free flight relevance. Interestingly, the cross-flow instability as a transition mechanism was first mentioned in King's 1992 paper [28], although cross-flow as factor in the location of turbulent transition was mentioned earlier [55]. The general observations in the supersonic cone experiments were that nose bluntness and wall cooling increases the transition Reynolds number, whereas angle of attack decreases the transition Reynolds number on the leeward side, and increases it on the windward side. Low disturbance environments seemed to increase the transition Reynolds number relative to higher disturbance free stream conditions.

The detailed description of the laminar-turbulent transition mechanism on the supersonic cone at an angle of attack is as follows. In general, the cross-flow growth rate is a maximum where the cross-flow Reynolds number is a maximum. For a cone at an angle of attack, the cross-flow Reynolds number increases and then decreases from the windward side of the cone to the leeward side. The max- 


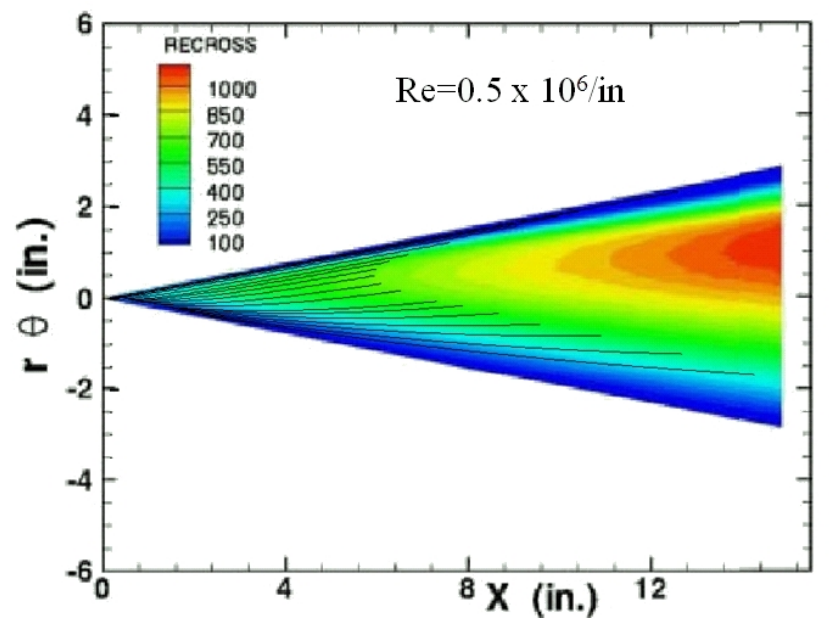

Figure 1.5. Cross-flow Reynolds number contours on the $7^{\circ}$ half angle cone at $4.2^{\circ}$ angle of attack. The black lines represent inviscid surface streamlines. Picture is adapted from Balakumar [3].

imum occurs towards an azimuthal location of about $130^{\circ}$, as measured from the bottom center of the cone. Cross-flow Reynolds numbers from Balakumar [3] for a $7^{\circ}$ half angle cone at a $4.2^{\circ}$ angle of attack are shown in Figure 1.5. Boundary layer profiles of the $\mathrm{u}$-velocity at different locations on the $7^{\circ}$ cone at a $4.2^{\circ}$ angle of attack are shown in Figure 1.6. An azimuthal angle of $\theta=0^{\circ}$ corresponds to the windward side, whereas the leeward side is designated as $\theta=180^{\circ}$. A thickening of the boundary layer from the windward side towards the leeward side can be seen. The boundary layer profiles are observed to become inflectional as the the leeward side at $\theta=180^{\circ}$ is approached. This is where the flow from both sides meet, and results in a relatively thick boundary layer.

Three different types of instability mechanisms are possible on a cone at an angle of attack in a supersonic flow: a viscous/TS-instability in the windward region, a three-dimensional cross-flow instability in the regions between the windward and leeward ray, and an inviscid/inflectional instability in the leeward region of the 


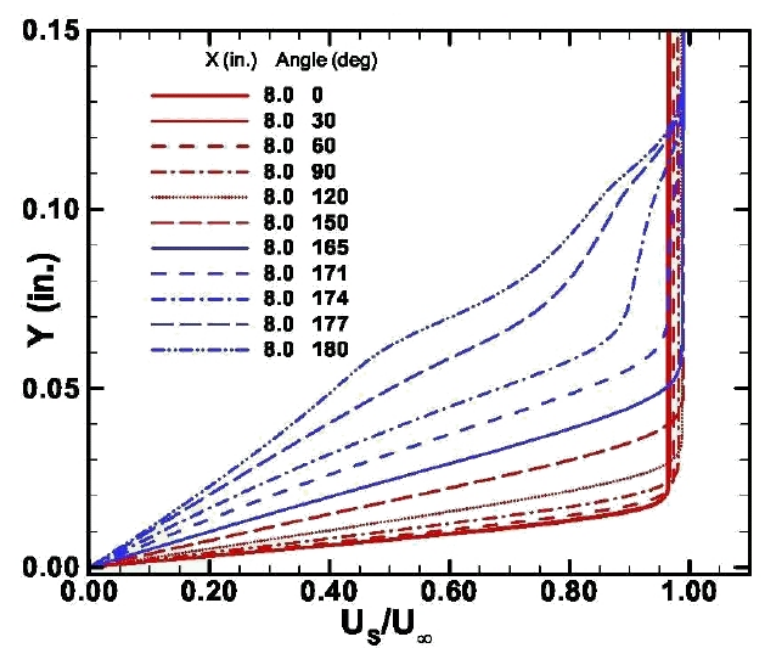

Figure 1.6. Boundary layer velocity profiles at constant axial location $20.3 \mathrm{~cm}$ ( 8 in) downstream from the cone tip at different azimuthal angles from $0^{\circ}$ to $180^{\circ}$. Picture is taken from Balakumar [3].

cone. The amplification rate of the TS-mode is usually much smaller than that of the cross-flow instability. The inflectional inviscid instability has the largest amplification rate. This has been shown in transition experiments by King [28] as well as N-factor calculations by Balakumar [3].

If the ratio of angle of attack $(\alpha)$ and cone half angle $(\theta)$ are chosen properly, the cross-flow mode will be the dominant transition mechanism over most of the cone surface. King [28] demonstrated that stationary cross-flow vortices were visible in the range between $\alpha / \theta=0.4$ to 0.8 .

There are no experiments where patterned distributed roughness has been used on a cone at supersonic Mach numbers to control cross-flow dominated transition to turbulence. 


\subsubsection{Choice of Wind Tunnel}

As explained in the previous sections, the free stream disturbance level dictates if stationary or travelling cross-flow modes will dominate in the laminar-turbulent transition process. It is understood that on aircraft at cruise altitude and Mach numbers, the free-stream disturbance conditions are relatively low in amplitude, between $\mathrm{u}^{\prime} / \mathrm{u}_{\infty}=0.03 \%$ and $0.005 \%$ according to measurements by Fisher et al. [18]. The NASA Mach 3.5 SLDT wind tunnel was designed to produce low disturbance conditions, particularly low acoustic disturbances. It is equipped with boundary-layer bleed slots upstream of the nozzle throat that remove the settling chamber boundary layer and its disturbances to help increase the boundary layer transition Reynolds number at the nozzle wall. The shape of the nozzle wall is a slow expansion design to minimize the growth of Görtler modes. The throat region is highly polished to remove roughness induced transition. The result of these steps is a low disturbance core in which a model can be located. More detailed information is given in Chapter 2, Other supersonic wind tunnels without these special design precautions have significantly higher background disturbance levels that are not as relevant to free flight conditions.

\subsubsection{DBD Plasma Bumps}

As part of the experiments, an array of Dielectric Barrier Discharge (DBD) plasma actuators was used as an equivalent replacement for a micron-sized roughness array. DBD plasma actuators are active flow control devices that use a non-thermal, weakly ionized plasma to assert a body force through electrohydrodynamical effects on neutral air molecules. The great advantage of DBD plasma actuators is that they have a high frequency response, they do not require any 
mechanically movable parts, and they have a very low power consumption in the range of several Watts per meter actuator [35]. Additionally they can be mounted flush with the surface, which is especially important for an application that is highly sensitive to 3-D surface roughness.

DBD Plasma actuators have been used in several active flow control scenarios over a wide range of Reynolds numbers. These range from separation control on airfoils and bluff bodies, to the excitation of viscous instabilities such as TS waves [20]. Reviews of the DBD plasma actuator and its applications were done by Corke et al. [13] and Moreau [35].

An isolated roughness element generates a horseshoe vortex that wraps around the base of the roughness element and extends downstream. The two arms of the horse-shoe vortex are counter rotating. They generate a local thinning and thickening of the boundary layer due to the entrainment of fluid in the wall-normal direction. If an array of roughness elements is used, a periodic thinning and thickening of the boundary layer can be generated which corresponds to the formation of positive and negative vorticity. The same effect can be achieved with a DBD plasma actuator array.

Some electrode geometries that have been used to generate streamwise vorticity are plasma jets [41] and streamwise vortex generator arrangements [12]. In 2001, Corke and Saric first demonstrated the excitation of cross-flow vortices on a swept wing using plasma streamwise vortex generators. The plasma actuation was designed to excite the critical wave number as well as the subcritical wavenumber. The boundary layer responded to both, which was documented by a measured periodic mean flow distortion.

In contrast to the DBD Plasma actuator as used in this work, there are several 
other plasma based techniques that use thermal effects to either generate expansion/compression waves, these include DBD Plasma actuator with nanopulses [36, 40], and filamentary arc discharges [30]. These techniques mainly work on producing localized heating, whereas the AC driven DBD Plasma actuator produces an electrohydrodynamic vector body force.

\subsection{Objective}

The delay of transition to turbulence on the highly swept wings of a supersonic aircraft will increase the supersonic cruise efficiency. This would help to achieve economically and environmentally feasible civil supersonic aircraft. The overall objective of this work is therefore to demonstrate that cross-flow dominated laminar-turbulent transition on highly swept surfaces in a Mach 3.5 flow can be delayed by means of subcritical forcing with an array of DBD plasma actuators. DBD plasma actuators will make it possible to quickly adapt to changing environmental conditions such as are expected to occur during a flight plan including take off, landing, and variations in cruising altitude.

The experiments will be conducted on a right-circular cone at angle of attack. The angle of attack will be chosen so that the dominant transition to turbulence of the boundary layer will be through a cross-flow instability. This will make it relevant to turbulent transition on a highly swept wing. The experiments will be conducted in the NASA Mach 3.5 SLDT using a slow expansion nozzle in order to have a low disturbance environment that will favor stationary cross-flow modes over the travelling cross-flow modes.

The following steps will be performed in order to achieve the overall objectives of this work: 
1. Establish that stationary cross-flow instabilities are responsible for laminarturbulent transition on the $7^{\circ}$ half-angle cone at a $4.2^{\circ}$ angle of attack at Mach 3.5.

2. Demonstrate using traditional micron-sized roughness arrays, that the stationary cross-flow instability is receptive to the forcing wavenumber at Mach 3.5 .

3. Demonstrate that subcritical forcing delays turbulent transition relative to the critical forcing condition at Mach 3.5.

4. Demonstrate that the stationary cross-flow instability is receptive to DBD plasma actuator arrays that simulate patterned roughness at Mach 3.5.

5. Demonstrate that subcritical forcing using a DBD plasma actuator array will delay turbulent transition relative to the critical forcing condition. 


\section{CHAPTER 2}

\section{EXPERIMENTAL SETUP}

\subsection{Wind Tunnel}

There are currently three low disturbance "quiet" tunnels in the U.S. which have different capabilities in terms of Mach number and run time. The only quiet tunnel that has the capability of extended run times is the NASA Langley Mach 3.5 Supersonic Low Disturbance Tunnel (SLDT). It is designed as a blow down facility and uses dry air that is filtered for particles larger than $1 \mu \mathrm{m}$. The tunnel exhausts to vacuum spheres, providing typical run times between 30 and 45 minutes. The stagnation pressures can be set to be from $34.5 \mathrm{kPa}$ to $1206 \mathrm{kPa}$ ( 5 to 175 psia) at a free stream Mach number of 3.5. The settling chamber includes seven turbulence management screens and five sintered mesh porous plates that are designed to attenuate incoming pressure fluctuations (acoustics). A specially designed axisymmetric nozzle is used to obtain a test zone with low free-stream disturbance levels. It has a highly polished, slow expansion contour to limit Görtler vortex formation on the nozzle wall. Additionally, boundary layer suction bleed slots are used at the nozzle throat to remove the turbulent boundary-layer which originates in the settling chamber and subsonic contraction. The boundary layer bleed slots can be opened or closed by valves which are connected through a circular concentric manifold to a vacuum reservoir [64]. A photograph of the bleed system 


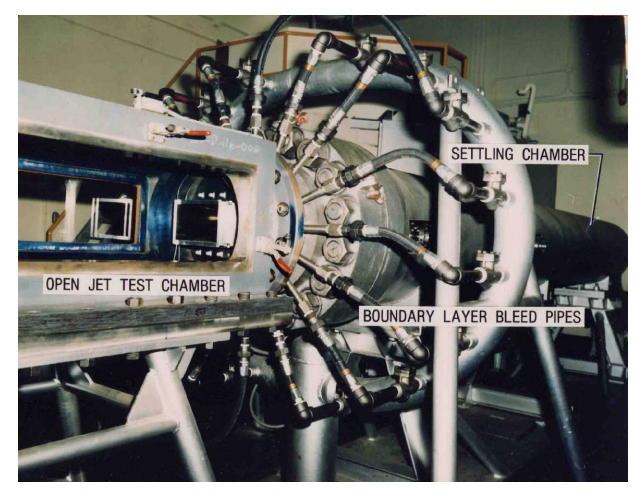

Figure 2.1. NASA Langley SLDT wind tunnel. View on test section and the bleed system (2D rectangular nozzle shown).

and test section is shown in Figure 2.1. The resulting constant Mach number quiet test core length, $\Delta x$, along the centerline of the nozzle depends on the total pressure $\mathrm{P}_{0}$. The cross section of the 3-D axisymmetric nozzle is shown in Figure 2.2. Hot wire measurements along the centerline of the nozzle at $\mathrm{P}_{0}=172 \mathrm{kPa}$ (25 psia) and $\mathrm{P}_{0}=345 \mathrm{kPa}(50 \mathrm{psia})$, and a total temperature of $\mathrm{T}_{0}=311^{\circ} \mathrm{K}$ show that the extent of the quiet core is $\Delta x=35.6 \mathrm{~cm}(14 \mathrm{in})$ and $\Delta x=27.9 \mathrm{~cm}(11 \mathrm{in})$, respectively. The quiet core length becomes smaller as a result of the transition to turbulence of the nozzle wall boundary layer at higher free stream Reynolds numbers. Typical pressure fluctuations in the quiet zone are lower than $0.08 \%$ of the free stream static pressure [26]. With the bleed valves open, the existence of Görtler vortices originating from the inside nozzle wall can be seen in Figure 2.3 . Providing that the boundary layer on the expanding nozzle is laminar, the quiet core is free of shocks. 


\section{Mach lines}

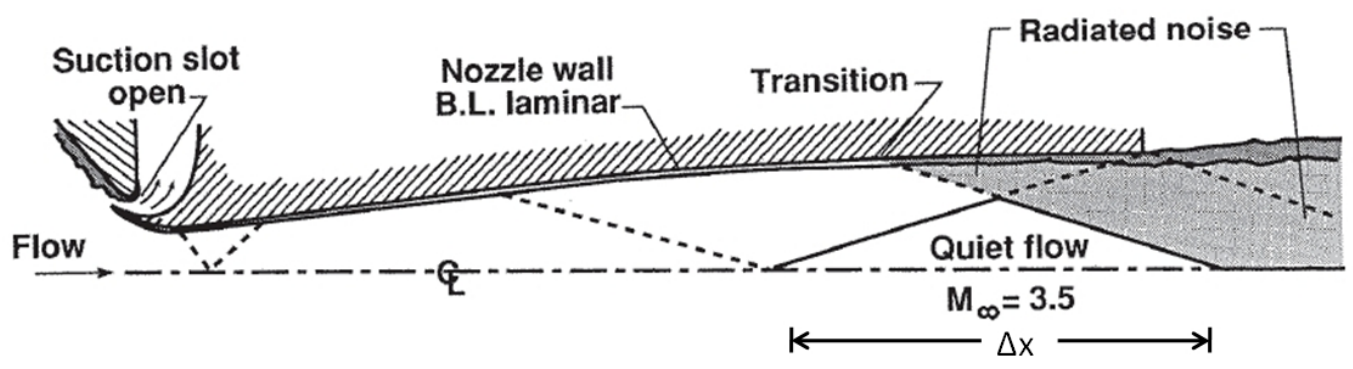

Figure 2.2. Cross section of the NASA Langley Mach 3.5 SLDT axisymmetric nozzle. The picture is showing the quiet test core and its constraints due to radiated noise.

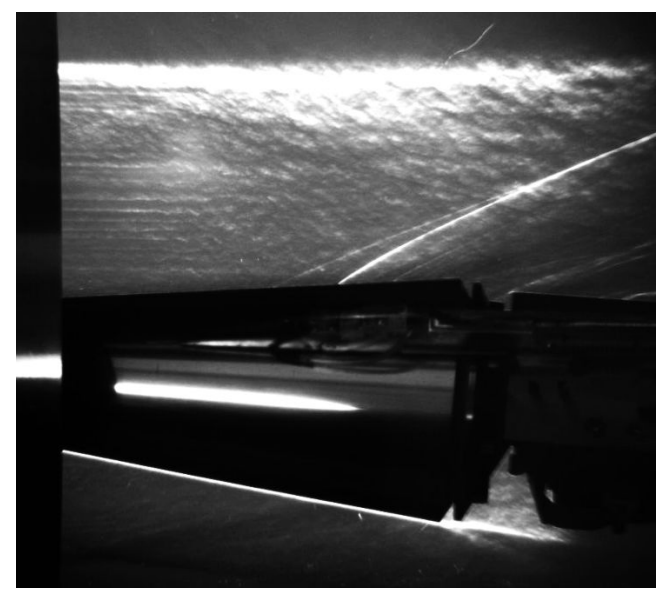

Figure 2.3. Schlieren image of the cone model inside the quiet core of the supersonic nozzle at Mach 3.5. Bleed valves are open and Görtler vortices are present. The quiet core is free of shocks. 


\subsection{Cone Model}

The cone-model used in the experiments is a hollow, thin-walled right circular cone with a $7^{\circ}$ half angle. It has a total length of 14 in or $35.56 \mathrm{~cm}$. It is made from 15-5ph stainless steel which was precipitation hardened to a H-1050 surface hardness, and polished to a mirror finish. The wall thickness over $85 \%$ of the cone surface is $2.03 \mathrm{~mm}(0.08 \mathrm{in})$. The other $15 \%$ of the cone model has a wall thickness of $0.76 \mathrm{~mm}(0.03 \mathrm{in})$ along a strip aligned in the flow direction. This strip contains thermocouples that were spot welded to the inner wall. 20 pressure taps with a diameter of $0.76 \mathrm{~mm}$ (0.03 in) were installed on the opposite side of the thermocouples. These are spaced $1.397 \mathrm{~cm}(0.55 \mathrm{in})$ apart and start $5.46 \mathrm{~cm}$ (2.15 in) downstream of the cone tip [9]. The maximum downstream variation in the cone radius per inch is 0.0002 in or $0.005 \mathrm{~mm}$, which corresponds to $1 \%$ of the minimum boundary layer thickness [34].

The base of the cone is mounted to a sting that is connected to a vertical strut on a base plate. The sharp tip of the cone is designed to be removable and threads into the front end of the cone body. This is shown in Figure 2.4. In order to generate a sufficient mean cross-flow, the cone was mounted at a $4.2^{\circ}$ angle of attack. The $\alpha / \theta$ ratio is 0.6 , which is proven to produce cross-flow Reynolds numbers that are large enough for the occurrence of cross-flow dominated transition [28].

\subsection{Probe Traversing Mechanism}

The probe traversing mechanism moves in wall-normal, axial and azimuthal directions. The whole mechanism was coaxially mounted to the sting of the cone with brass sleeve bearings. A stepper motor with a gear box rotates the traversing mechanism around the sting. It is indicated as "Azimuthal Motion" in Figure 2.5. 


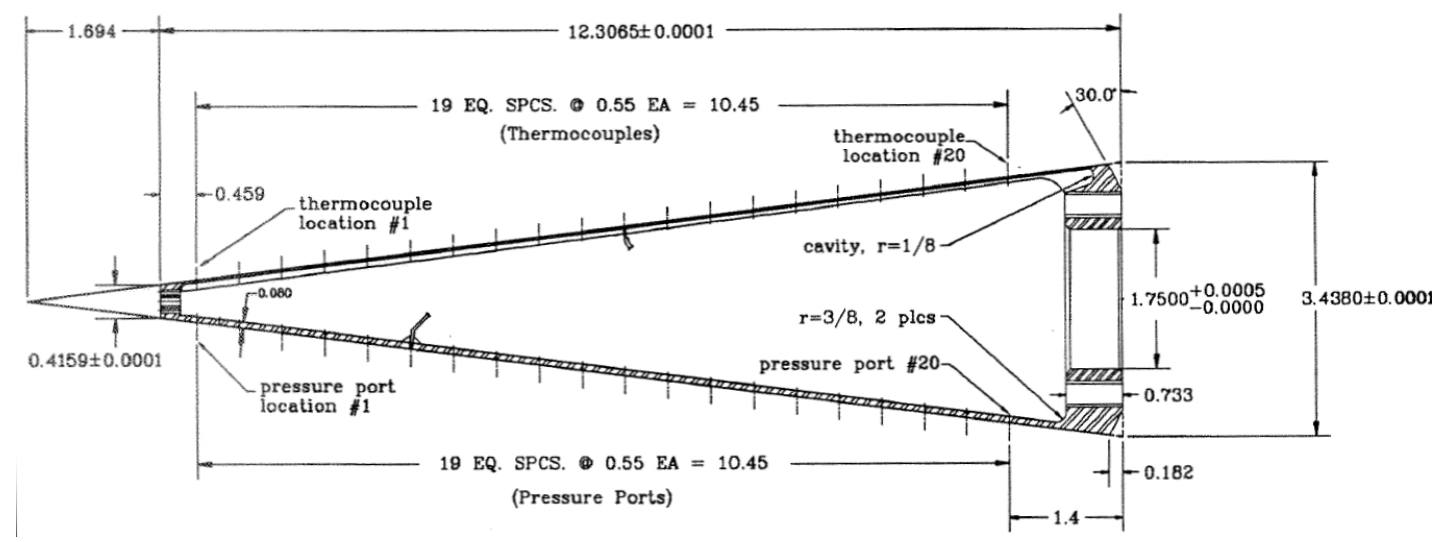

Figure 2.4. Cross section of the $7^{\circ}$ half angle cone with dimensions including the mounted removable cone tip. The drawing does not include the axial mounting sting. Units are in inches.

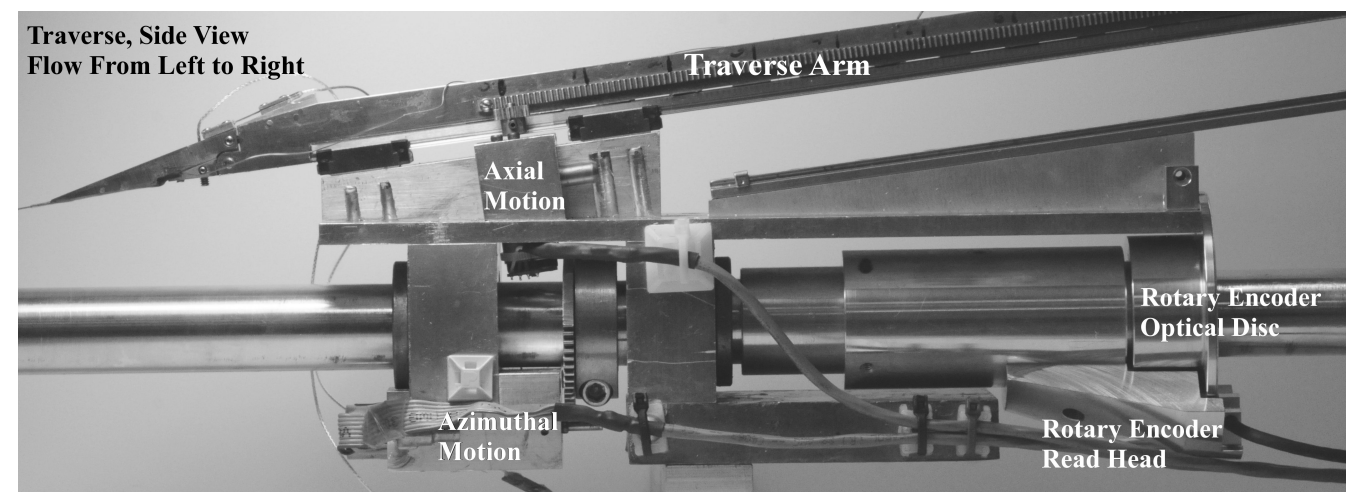

Figure 2.5. Traverse seen from the side. 
The arm that carries the Pitot tube is mounted on a linear slide. It is moved with another stepper motor in the axial direction. This is again indicated in Figure 2.5. The tip of the probe arm is connected with a hinge pin to a steel connector piece that holds a sensor for the estimation of its wall-normal position. The Pitot tube is integrated into a steel wedge that can be mounted with a screw on the connector piece. This is shown in the photograph in Figure 2.6. Because the tip of the traverse arm can rotate around the hinge pin, an internal lead screw is used to push or pull it in the axial direction, above the center of rotation. By doing so, the height of the probe above the cone surface can be controlled. The lead screw is connected to another stepper motor with a gear box that is mounted at the rear end of the traverse arm. It moves fore and aft with the arm.

The traverse is also equipped with a Numerik Jena ${ }^{1}$ rotary encoder to detect the azimuthal position. This is shown on the traverse in Figure 2.5 and in the schematic in Figure 2.7. It utilizes an optical disk with 2056 lines that are read by an encoder that includes a $1 / 5$ line interpolation electronics that generates a RS255 output signal. The resolution of the encoder is $0.035^{\circ}$. The wall normal position of the Pitot tube was measured with a differential variable reluctance sensor $^{2}$ (DVRT), or also known as half bridge linear variable displacement sensor (LVDT), that detects the relative rotation between the traverse arm and the pivoting wedge. The LVDT consists of a $1.8 \mathrm{~mm}$ outer diameter cylindrical sensor body and a $0.8 \mathrm{~mm}$ outer diameter titanium sliding core. A photograph of the LVDT sensor is shown in Figure 2.8. The sliding core is attached to the pivoting wedge, and the sensor body is mounted on the traverse arm. It then detects the relative motion between the traverse arm and the pivoting wedge.

\footnotetext{
${ }^{1}$ Numerik Jena GmbH, D-07743 Jena, Germany

${ }^{2}$ Microminiature DVRT ${ }^{\circledR}$ by Microstrain Inc, Williston, VT, USA
} 


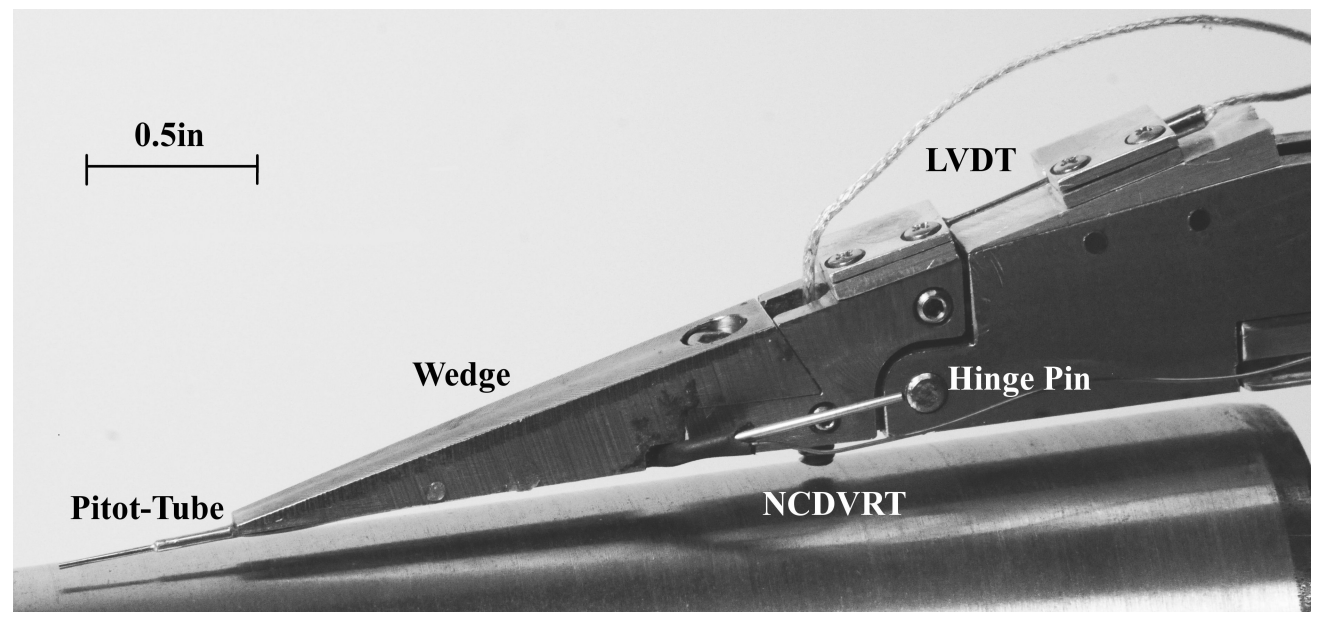

Figure 2.6. The tip of the traverse arm: Removable wedge with Pitot tube and LVDT sensor for positioning.

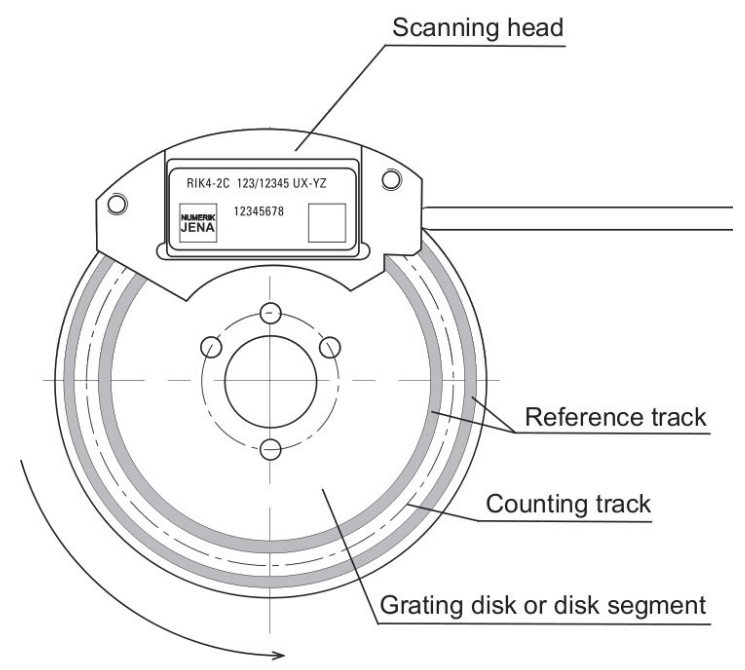

Figure 2.7. Schematic of the optical rotary encoder RIK 2C 64/2048 L1-W3 from Numerik Jena. 


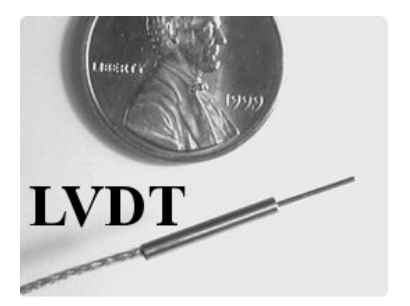

Figure 2.8. LVDT displacement sensor for the wall normal motion of the wedge and Pitot tube.

The azimuthal and wall-normal position sensors are used as proportional feedback sensors for the two respective motions. The axial motion stepper motor on the other hand, is operated in an open loop, without position feedback. The axial stepper motor is equipped with a zero backlash gear box. The pinion gear which moves the axial arm however, has a small amount of backlash. Because the axial motion is facing a constant drag force by the supersonic flow during tunnel operation, the axial motion arm is constantly being pushed against the teeth of the axial motion pinion gear. This effectively removes the backlash in the axial motion.

\subsubsection{Calibration Procedure and Estimation of Uncertainty for the LVDT Sensor}

A Keyence ${ }^{3}$ LS optical micrometer was used to calibrate the LVDT sensor. A $249 \mathrm{~mm}$ long Balsa wood rod was connected to the steel connector piece at the tip of the traverse arm. The up and down motion of the tip piece was amplified by the wood rod. The tip of the Balsa wood rod generated a shadow in the measurement field of the optical micrometer that was directly translated into an absolute position. The repeatability of the micrometer was given with $\pm 0.2 \mu \mathrm{m}$. The ac-

\footnotetext{
${ }^{3}$ Keyence Corp of America, Woodcliff Lake, NJ, USA
} 
curacy was $\pm 3 \mu \mathrm{m}$. The calibration curves for the LVDT are shown in Figure 2.9. A second-order polynomial fit of the LVDT calibration showed a $95 \%$ confidence interval of $\pm 36 \mu \mathrm{m}$ or $0.26 \%$ of the range. The combination of the first order uncertainty from the micrometer and the calibration curve is approximately $36.1 \mu \mathrm{m}$ at the tip of the Balsa calibration rod. The resolution of the LVDT itself as given by the manufacturer, is $\pm 0.3 \mu \mathrm{m}$ with a repeatability of $\pm 1 \mu \mathrm{m}$. The manufacturer accuracy is $\pm 0.1 \%$ of the measurement range. With this information, the first order uncertainty of the LVDT sensor was calculated for the displacement measurement of the calibration arm tip. Knowing the radial distance from the hinge pin to the LVDT, the error in displacement at the calibration arm tip including the manufacturer given values is then $87 \mu \mathrm{m}$. The calculation of this value is shown in Appendix A.

To derive the uncertainty at the Pitot probe tip location, the ratio between the Pitot probe length relative to the hinge pin, and the Balsa wood length was used. The uncertainty of the Pitot probe location was then $23 \mu \mathrm{m}$ or $1.8 \%$ of the wall-normal motion range of 0.05 in or $1.27 \mathrm{~mm}$.

\subsubsection{Offset Estimation Procedure}

In order to estimate the offset of the LVDT calibration curves, a so called "touch" circuit was used. Because the Pitot tube was electrically insulated from the traverse, it could be used as a contact switch by triggering an electronic circuit when it touched the cone surface. The cone surface could then be used as a natural zero reference point for the Pitot probe positioning. 


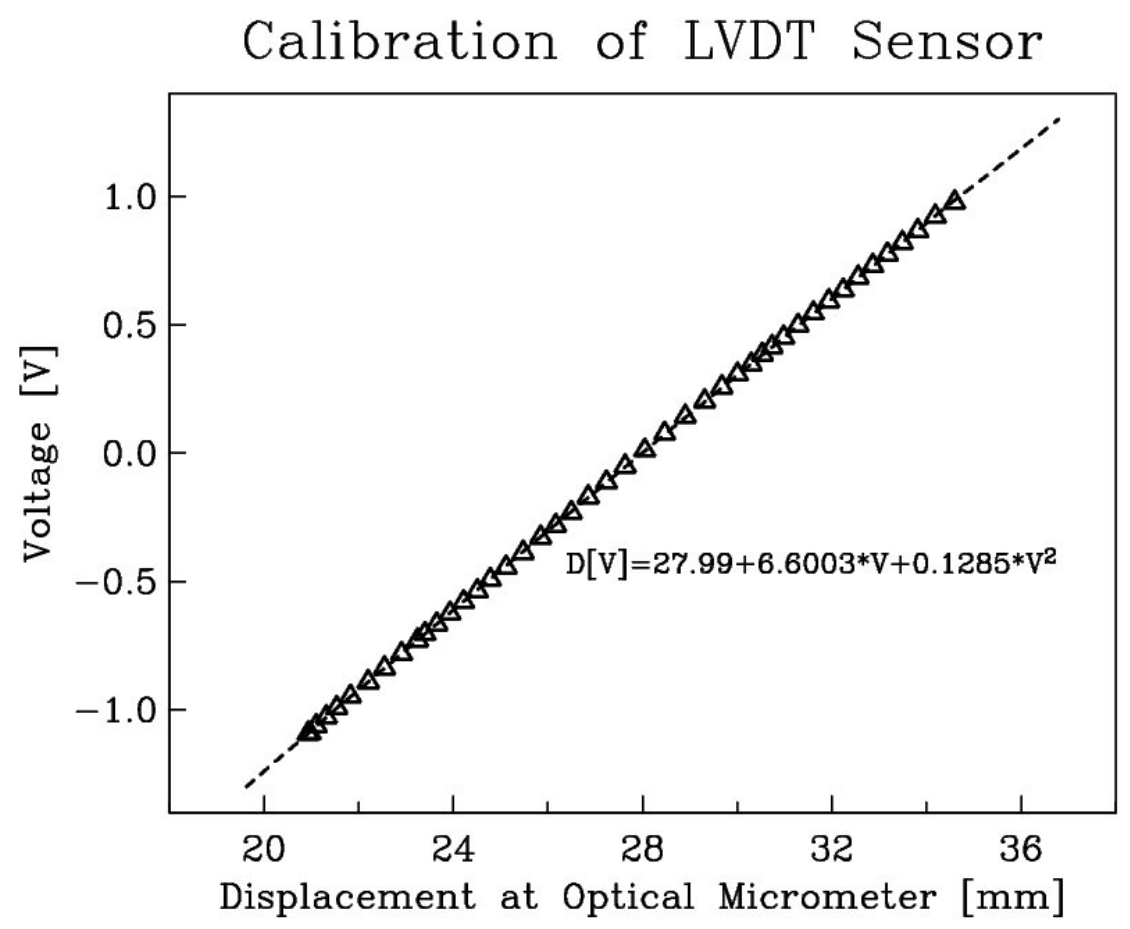

Figure 2.9. Calibration curve for the LVDT sensor. 


\subsubsection{Pitot Probe}

Two different Pitot probes were used for the measurements. The first Pitot probe was made of a hypodermic steel tube with $0.89 \mathrm{~mm}$ outer diameter. The tip was flattened to an oval shape to increase the vertical resolution in the thin boundary layer of the cone. The oval height was $0.46 \mathrm{~mm}$ and its width was $1.2 \mathrm{~mm}$. The length of the Pitot tube was $12.5 \mathrm{~mm}$.

The second Pitot probe had the same length but an inner diameter of $0.25 \mathrm{~mm}$ and an outer diameter of $0.5 \mathrm{~mm}$. This allowed for a better spatial resolution and less flow interference, as shown by Matlis [34]. The chosen Pitot probe dimension was a compromise between minimized flow interference, maximized spacial resolution, and pressure lag time. Using smaller probe diameters than the chosen one, increased the pressure lag time of the measurement system and became therefore unpractical for taking data series with limited wind tunnel run times. The length of the tubing that connected the Pitot tube to the pressure transducer was approximately $1 \mathrm{~m}(3 \mathrm{ft})$ and had an inner diameter of $1.2 \mathrm{~mm}(0.05 \mathrm{in})$. At the most upstream position of the Pitot probe tip, the minimum wavelengths of the stationary cross-flow modes were expected to be approximately $0.9 \mathrm{~mm}$ based on the local cone circumference at $\mathrm{x}=8.89 \mathrm{~cm}(3.5 \mathrm{in})$ and an azimuthal wavenumber of $m=68$. An inner diameter of the Pitot probe of $0.25 \mathrm{~mm}$ was about one quarter of the shortest expected azimuthal wavelength, and should therefore be sufficient to resolve the wavenumber content of the expected stationary cross-flow modes.

The static pressure ports on the cone, stagnation pressure, and the Pitot probe pressure were measured with a Baratron ${ }^{4} 690$ pressure transducer. This was used with a MKS Type 670 signal conditioner. The transducer and signal conditioner

\footnotetext{
${ }^{4} \mathrm{MKS}$ Instruments, Androver, MA
} 
had current calibrations to factory specifications of $0.08 \%$ of the reading.

\subsection{Data Acquisition}

The data acquisition was done with a PMCCorp ${ }^{5}$ motion control card that included 8 analog input channels. This was used to monitor the total pressure of the Pitot tube, the static pressure at the surface of the cone, the surface contact circuit, the LVDT-Demodulator output, and the temperature in the test section. The software to control the traverse motion and data acquisition was written in C.

\subsection{Numerical Stability Analysis}

As described in the introduction, the choice of the wave number and location of the initial disturbance was crucial to the successful application of subcritical forcing. Dr. P. Balakumar from the NASA Langley Research Center, Flow Physics and Control Branch provided a stability analysis of the cone model at the experimental conditions. He solved the full compressible Navier Stokes equations with a 5th order weighted essentially non-oscillatory (WENO) scheme for spatial discretization, and a third order total-variation-diminishing (TVD) scheme for temporal integration. The linear stability analysis was then performed assuming local parallel theory. Further details are given in [3].

Linear stability analysis predicts the location of Branch I and the wavenumbers of the most amplified stationary cross-flow modes. Growth rates for the most amplified stationary cross-flow modes along rays of constant azimuthal angle at $45^{\circ}, 90^{\circ}$ and $120^{\circ}$, as depicted in Figure 2.10, are shown in Figure 2.11 as solid

\footnotetext{
${ }^{5}$ Precision MicroControl Corp., Carlsbad, CA 92011
} 


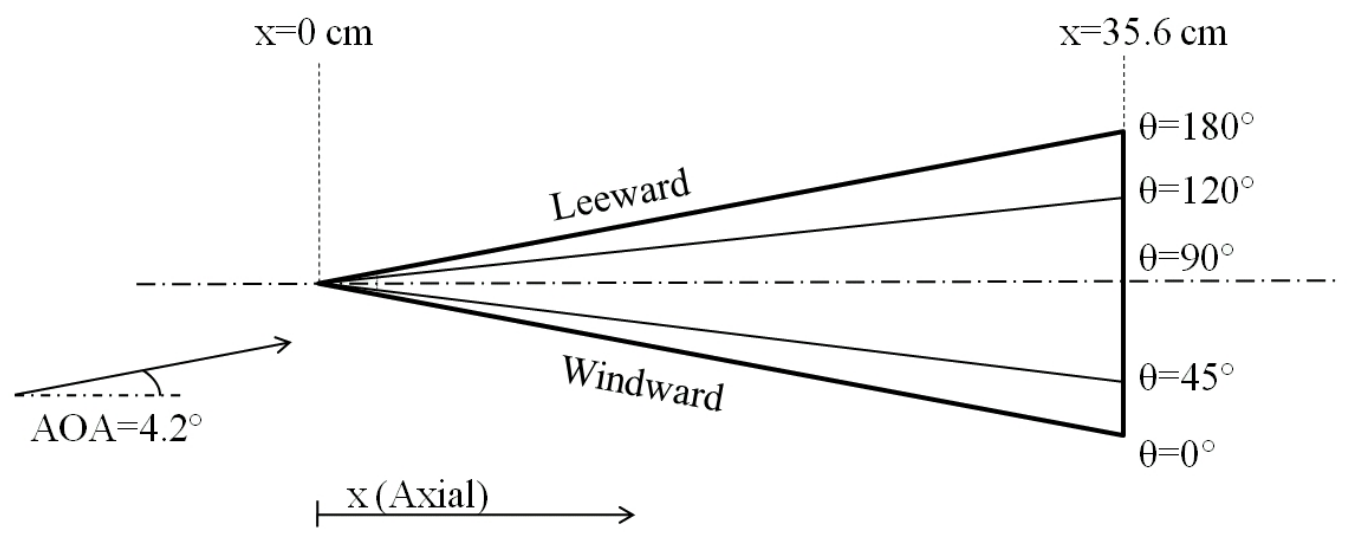

Figure 2.10. Rays of constant azimuthal angle on the cone.

and dashed blue lines with square markers. The stationary cross-flow modes begin to be amplified near the axial location of $1.27 \mathrm{~cm}(0.5 \mathrm{in})$. Therefore a location of $\mathrm{x}=1.27 \mathrm{~cm}(0.5 \mathrm{in})$ was chosen for the patterned roughness. This ensured that the roughness forcing occurred, upstream of the axial location of Branch I.

The azimuthal wavenumber of the most amplified stationary cross-flow mode based on the analysis was close to 50 for each Reynolds number case. Based on this, $m=45$ was chosen as the controlled critical wavenumber. The subcritical wavenumber was defined as a wavenumber that was 1.5 times larger that the critical wavenumber. Therefore the subcritical wave number for the experiment was $m=68$.

\subsection{Disturbance Generators}

The exchangeable cone tips were made of Polyamide-imide or Torlon ${ }^{6} 4301$ resin, which is electrically insulating and possesses a high tensile strength of

\footnotetext{
${ }^{6}$ Trademark of Amoco Performance Products, Inc., Alpharetta, GA
} 

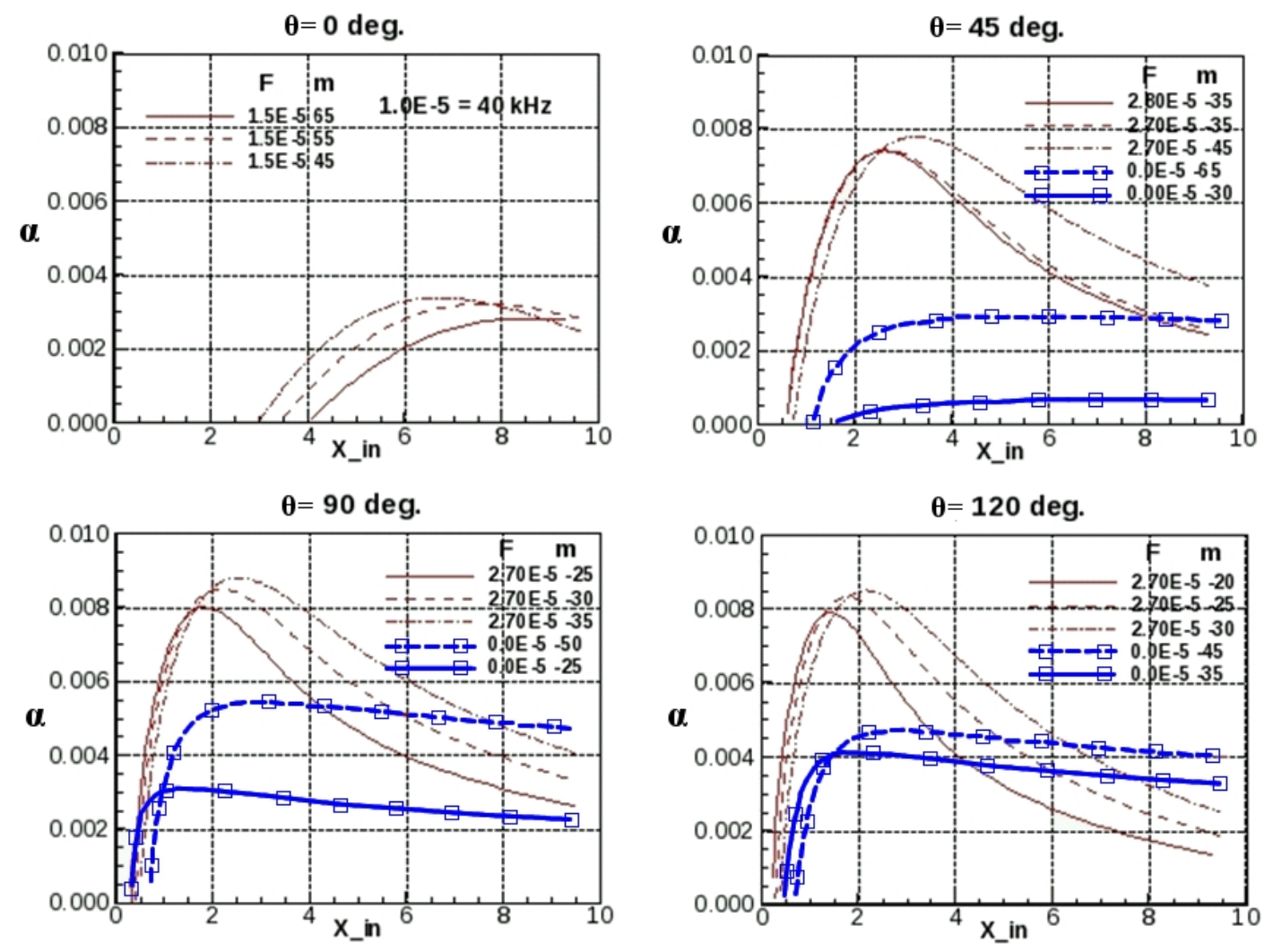

Figure 2.11. Growth rates for axial rays at constant azimuthal angle $\theta$ obtained from Balakumar's linear stability calculations 3 at

$$
R e_{\infty}=9.8 \times 10^{6} \mathrm{~m}^{-1}\left(250,000 \mathrm{in}^{-1}\right) .
$$


$82 \mathrm{MPa}$. A non-conductive material was chosen to allow for the future use of the tips with plasma generators on the surface. The nose tip radius was between $0.2 \mathrm{~mm}$ and $0.25 \mathrm{~mm}(0.008 \mathrm{in}$ and $0.01 \mathrm{in})$.

\subsubsection{Passive Mechanical Dimples}

Two different passive roughness cone tips were made. These had 45 and 68 equidistant dimples around the circumference that were located $1.27 \mathrm{~cm}(0.5 \mathrm{in})$ downstream of the cone tip. The dimples were produced by pressing a sharp needle into the Torlon surface. The number of dimples corresponded to the wavenumber, m. A Keyence Model VHX-600 3-D Imaging microscope was used to quantify the depth and diameter of the dimples. The dimples on the $m=45$ tip were slightly oval, with an axial length of $180 \mu \mathrm{m}$ and an azimuthal width of approximately $145 \mu \mathrm{m}$. The depth of the dimples was $39 \mu \mathrm{m}$. The wavelength to azimuthal width ratio was 0.65 . The diameter of the $m=68$ dimples was approximately $80 \mu \mathrm{m}$. This gave a diameter to wavelength ratio of approximately 0.6. In both cases, the minimum diameter to wavelength ratio of 0.5 for effective forcing recommended by Saric and Radeztsky [38, 42] was exceeded.

A third cone tip made of polished steel with a mirror finish, was used as the baseline reference case. It was denoted as $m=0$.

\subsubsection{Plasma Disturbance Generators}

Two different approaches were used to fabricate a controlled wavenumber plasma roughness disturbance generator for the tip of the cone. One used individual circular plasma dots, that were designed to act like miniature wall jets. The other design consisted of a serrated edge that was designed to generate streamwise 

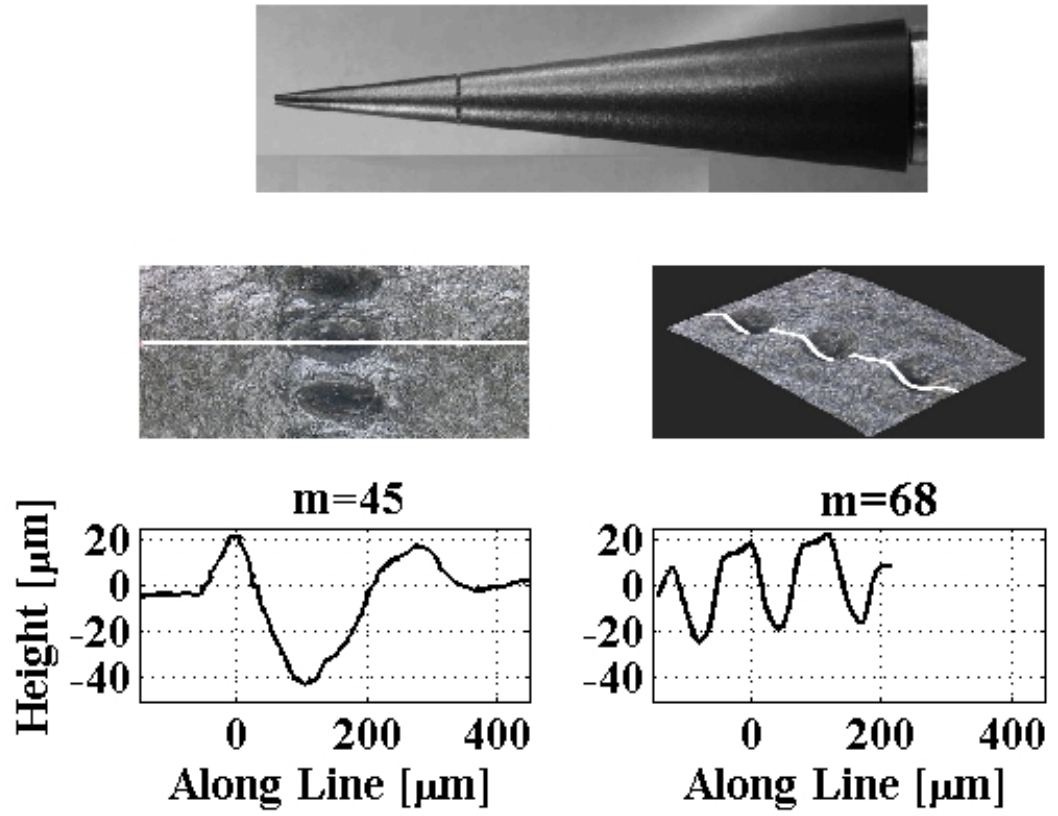

Figure 2.12. Photographs of the $m=45$ cone tip, and magnified views of the $m=45$ and $m=68$ dimples, including the topography across the dimples. 
vorticity. There were two main challenges in the design of these plasma actuators. The first was the need to not introduce any passive roughness effects. The second was that there be sufficient space between the electrode to generate the plasma. The design and fabrication of the actuators under these challenging constraints, on a curved surface, is described in the next sub-sections.

\subsubsection{Individual Circular Plasma Dots}

The fundamental idea of the plasma dots was to generate plasma in localized spots that would simulate the effects of the roughness dimples. The standard DBD plasma actuator arrangement consists of two asymmetrically arranged electrodes separated by a dielectric layer. A schematic view of a typical plasma actuator is shown in the left or right half of cut $\mathrm{A}-\mathrm{B}$ in Figure 2.13. If an $\mathrm{AC}$ voltage with sufficient amplitude is applied, a weakly ionized gas forms at the edge of the exposed electrode. The charged particles exchange momentum with the neutral ambient air. This can be modeled as a body force vector field directed away from the exposed electrode edge towards the covered electrode. The momentum flux produces a tangential wall jet.

To generate a pattern of individual circular plasma zones, circular holes were removed from the exposed electrode. Plasma formed at the inside edges of the hole when an AC high voltage signal was supplied to the electrodes. The body force in this geometry was then directed towards the center of each hole. This produced a stagnation point at the center of the holes that caused the flow to jet away from the wall at that point. This is illustrated in the schematic shown in Figure 2.13. 

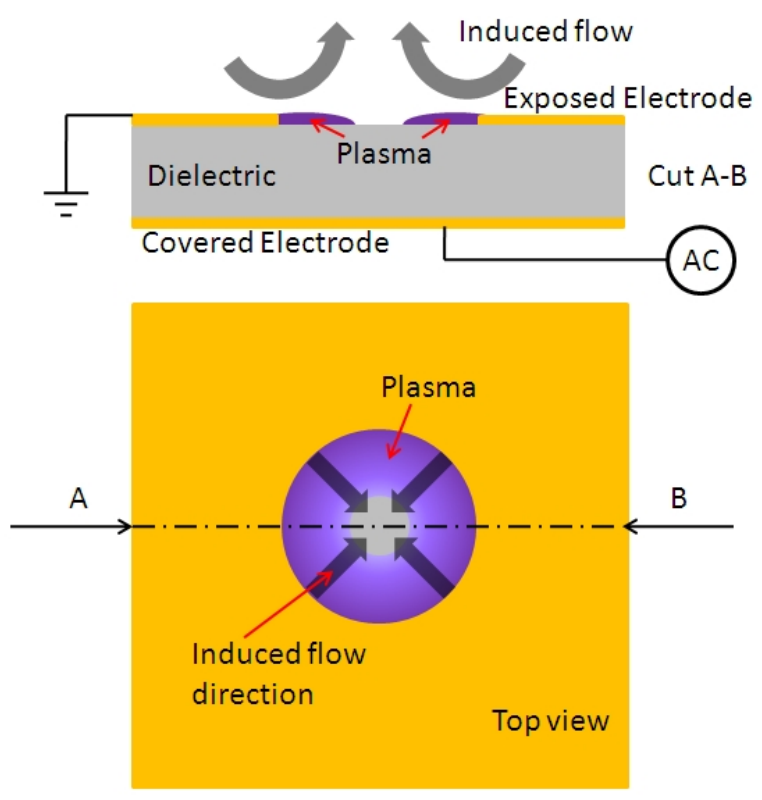

Figure 2.13. Schematic drawing of a single plasma dot actuator arrangement including the induced flow direction (not to scale). 


\subsubsection{Serrated Edge / Comb actuator}

The second electrode pattern was designed as a comb structure that was similar to the streamwise vortex generators used by Corke and Saric in 2001 for excitation of stationary cross-flow modes on a swept wing model. That design used by Corke and Saric is shown in the left part of Figure 2.14. The present design is shown on the right hand side of the figure. The body force is normal to the flow direction. The induced flow stagnates along a line half-way between the inside edges of two neighbor electrodes. This results in a jetting of the fluid away from the wall that rolls up into a streamwise vortex. The symmetry of the electrodes results in pairs of counter-rotating vortices that are centered on each electrode. This is indicated in the schematic on the left part of Figure 2.14. A detailed analysis of this flow field is presented in Chapter 3 .

\subsubsection{Vapor Deposition}

The challenge of flow actuators used in cross-flow experiments is the very high sensitivity of the cross-flow instability to surface roughness. The patterned surface electrodes had to be smooth enough to disappear within the natural random surface roughness. Otherwise the targeted cross-flow modes would be excited no matter if the plasma was turned on or off.

One method of generating a smooth surface, in spite of the holes in the exposed electrode, was by using a very thin electrode material. The electrode material that was chosen was copper. Besides being a good electrical conductor, it could be etched off easily. Copper has a resistivity of $\rho=1.68 \times 10^{-8} \Omega \mathrm{m}$. Given a thickness $t$, width $W$, and length $L$ of the electrode, the resistance of the copper electrode is $R=\rho L / t W$. Assuming that $L=2.79 \mathrm{~cm}(1.1 \mathrm{in})$ and $W=0.74 \mathrm{~cm}$ 

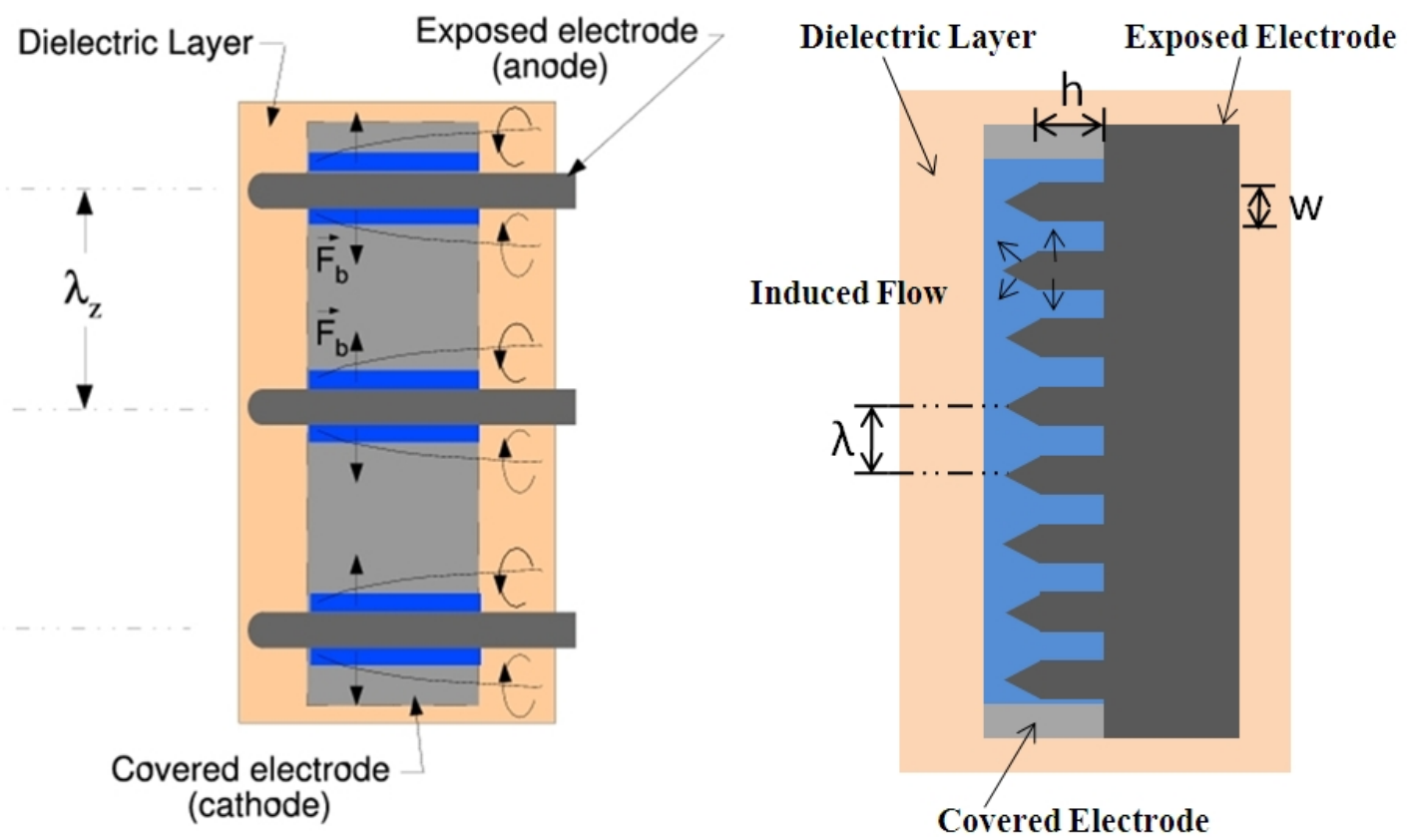

Figure 2.14. Left hand side: Schematic of the streamwise vorticity generator as used by Saric and Corke in 2001. Right hand side: Drawing of the comb actuator geometry as used in the current supersonic experiments. $\lambda$ is the wavelength of the desired stationary mode. $h=\lambda$ and $w=\lambda / 2$ 
(0.39 in) (circumference at the plasma array at $\mathrm{x}=1.27 \mathrm{~cm}(0.5 \mathrm{in}))$ a copper sheet with a thickness of only $47 \mathrm{~nm}$ has a resistance of less than $1 \Omega$.

One of the most common methods of generating a thin layer of a copper material is by vapor deposition. The sample to be coated was placed inside an ultra-high vacuum chamber of an electron beam evaporator, facing a small crucible filled with solid copper. An electron beam was focused onto the copper, which heated it up so that it liquefied. The copper slowly evaporated and condensed on all the surfaces that were in direct line of sight of the vaporized copper. The deposition rate and final thickness could be accurately monitored with a thin film deposition controller.

\subsubsection{Kapton Film Actuator}

A few test actuators were made on $12.7 \mu \mathrm{m}(0.5 \mathrm{mil})$ and $25.4 \mu \mathrm{m}$ (1 mil) thick Kapton $^{7}$ film. The process of fabrication consisted of multiple steps: mask making, photo-lithography, vapor deposition, and lift-off.

The mask was designed with a CAD software. It was then translated into a set of equivalent squares that were loaded into the mask making machine control software. The machine then exposed the pattern into a chromium emulsion on a glass carrier using UV light.

In the first step of the process, Kapton film was attached to a $120 \mathrm{~mm}$ diameter Silicon wafer with thermal release tape $\left(\right.$ Revalpha $\left.^{8}\right)$. Thermal release tape loses its adhesion when heated up above a certain temperature. If the tape was just standard pressure sensitive glue, there would be a good chance that the deposited copper would peel off when being removed from the Silicon wafer carrier. The

${ }^{7}$ Trademark of E.I. DuPont de Nemours and Co, Wilmington, DE

${ }^{8}$ Nitto Americas, Inc., Teaneck, NJ 07666, U.S.A. 
wafer itself was used to hold the Kapton film for further processing in standard machines for micro-electronic fabrication. In the second step, the surface of the Kapton film was coated with a thin layer of $\mathrm{AZ}^{9} 5214$ negative photoresist in a spin coater. The resist was then exposed to UV light using a mask that was made in the previous step.

After developing the photo-resist, there was a series of circular islands left on the surface of the Kapton. Copper was next deposited on the surface with the photo-resist islands. After deposition, the copper layer was cleaned with acetone, which dissolved the photo-resist under the copper and removed the copper on top of the photo-resist residues. The result is shown in the photograph in Figure 2.15. This technique is also known as "lift off" patterning.

In the last step, the wafer was heated to release the Kapton film. The Kapton film was then glued again onto a silicon wafer with thermal release tape. This time, the copper was facing the wafer. The "covered" electrode was then vapor deposited on the upward facing side of the Kapton film. Afterwards it was released from the Silicon wafer using heat.

The Kapton film with the exposed and covered electrodes was cut into a pielike shape and then wrapped around the cone tip. A two component epoxy was used to bond the Kapton film to the cone tip surface.

The cone tip itself was made of Torlon. It was designed with a hollow center. A hole was drilled through to the surface to make an opening for a thin wire. The wire was soldered to the covered electrode with low temperature solder. The low temperature solder (TIX solder $\left.{ }^{10}\right)$ melts at $408^{\circ} \mathrm{K}\left(275^{\circ} \mathrm{F}\right)$. When higher temperatures were applied to the deposited copper film, the copper immediately peeled

\footnotetext{
${ }^{9}$ Clariant Corporation, Somerville NJ 08876

${ }^{10}$ Allied MFG. Corp., Bozeman, MT
} 

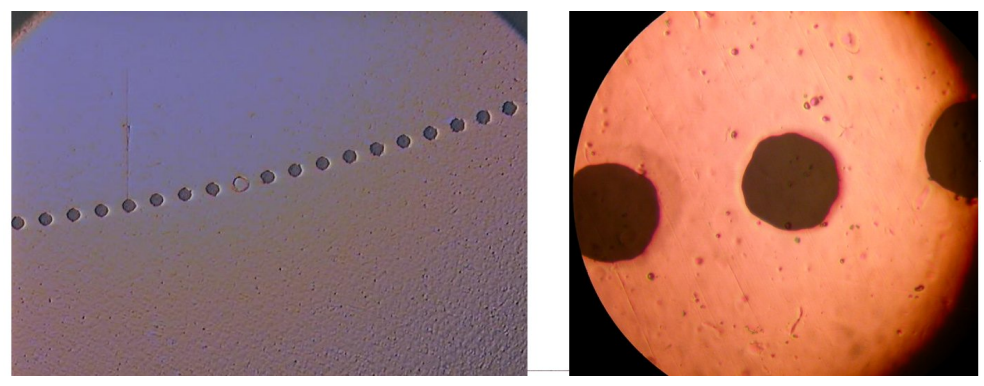

Figure 2.15. Enlarged image of the exposed electrode after lift off. Each dot has a diameter of $76 \mu \mathrm{m}$ (0.003 in) and is spaced approximately $152 \mu \mathrm{m}(0.006 \mathrm{in})$ apart. The substrate is a $12.7 \mu \mathrm{m}(0.5 \mathrm{mil})$ thick Kapton film.

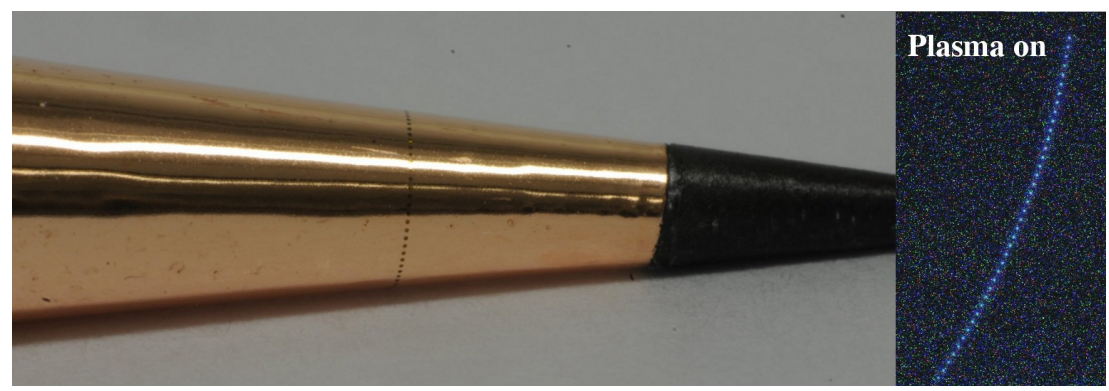

Figure 2.16. $12.7 \mu \mathrm{m}$ (0.5 mil) thick Kapton actuator glued to a Torlon carrier cone. Right hand side picture shows the operating plasma dots at $10.2 \mathrm{kPa}(3 \mathrm{inHg})$ ambient pressure with $2.2 \mathrm{kVpp}$ and $3 \mathrm{kHz}$ sin-wave. 
off of the Kapton.

Although the plasma dots generated plasma as planned, it was extremely difficult to glue the Kapton film actuator onto the surface of the Torlon cone tip without adding steps or other unwanted surface imperfections. It was not possible to wrap the Kapton film around the cone without leaving a small gap where both edges met. The joint that formed at the upstream edge of the Kapton film was a step that was a significant source of surface roughness. This was a critical concern due to its proximity to Branch I of the neutral growth curve.

A photograph of the glued Kapton film is shown in Figure 2.16. The right photograph in Figure 2.16 shows the plasma dots operating. The surface step caused by the Kapton film can be clearly seen in the left photograph. Therefore, the Kapton film approach was not pursued further. Instead, a design that used the cone tip as the dielectric was developed. The description of the process to fabricate this design is given in the following section.

\subsubsection{Dielectric Cone Tip Actuator}

In order to avoid the difficult process of wrapping and gluing the Kapton film onto the cone tip surface, the cone tip structure itself was designed to act as the dielectric. The cross section of the final design is shown in Figure 2.17. The cone tip consisted of two materials, Macor ${ }^{11}$ towards the tip, and aluminum at the base. The tip itself was made in several steps. First a $7^{\circ}$ tapered end mill was used to drill a tapered hole into a $9.53 \mathrm{~mm}(3 / 8 \mathrm{in})$ diameter Macor rod. Next a $7^{\circ}$ taper was cut into the end of an aluminum rod that had a through hole in the center to allow the electrical connection to the covered electrode. Conductive Silver epoxy

\footnotetext{
${ }^{11}$ Corning SAS, 77211 Avon Cedex France
} 
(Loctite $^{12} 3888$ ) was used to fill the tapered hole of the Macor rod to act as the covered electrode. The silver epoxy also made the connection to the high voltage wire $^{13}$. The wire was insulated by an FEP (Fluorinated Ethylene Propylene) coating. It had an outer diameter of $1.27 \mathrm{~mm}(0.05 \mathrm{in})$, and a dielectric strength of $14 \mathrm{kVrms}$. The high voltage wire passed through the hole in the aluminum base of the tip. The tapered aluminum end and the tapered Macor tip were brought together and bonded with a fast curing two-component epoxy (Loctite 608). The joined parts were then machined on a lathe into the final conical shape. The wall thickness at the Macor tip was approximately $0.38-0.51 \mathrm{~mm}(15-20 \mathrm{mil})$ and the nose tip radius was in the order of $0.025 \mathrm{~mm}(0.001 \mathrm{in})$. The entire surface of the cone tip was then smoothed with sandpaper (grit size 280 to 1800), and with lapping compound (equivalent grit size 3200). A wall thickness of about $0.38-0.51 \mathrm{~mm}(15-20 \mathrm{mil})$ resulted in a dielectric strength of $12-15 \mathrm{kVpp}$ [50].

The exposed electrode was made by depositing copper onto the outer surface of the cone tip, on top of the Macor and aluminum parts of the cone tip. In doing so, a continuous copper layer was electrically connected to the metal base of the cone tip, which was in turn connected to the metal cone which was electrically grounded. The electrode pattern was then etched into the exposed copper layer.

The surface curvature of the cone tip was a big challenge for photo-lithography. Neither of the standard techniques, spin coating and a flat mask could be directly applied. A solution was found by using positive MicroSpray spray-on photo-resist from Microchem ${ }^{14}$. It comes in a can, and could be easily sprayed onto the surface of the cone tip. The best result was obtained by spraying one passage every $45^{\circ}$

\footnotetext{
${ }^{12}$ Henkel AG \& Co. KGaA, D-40589 Düsseldorf

${ }^{13}$ ETN-2026-10, Connectronics Corp, Toledo, OH-43607, USA

${ }^{14}$ Microchem Corp., Newton, MA 02464
} 


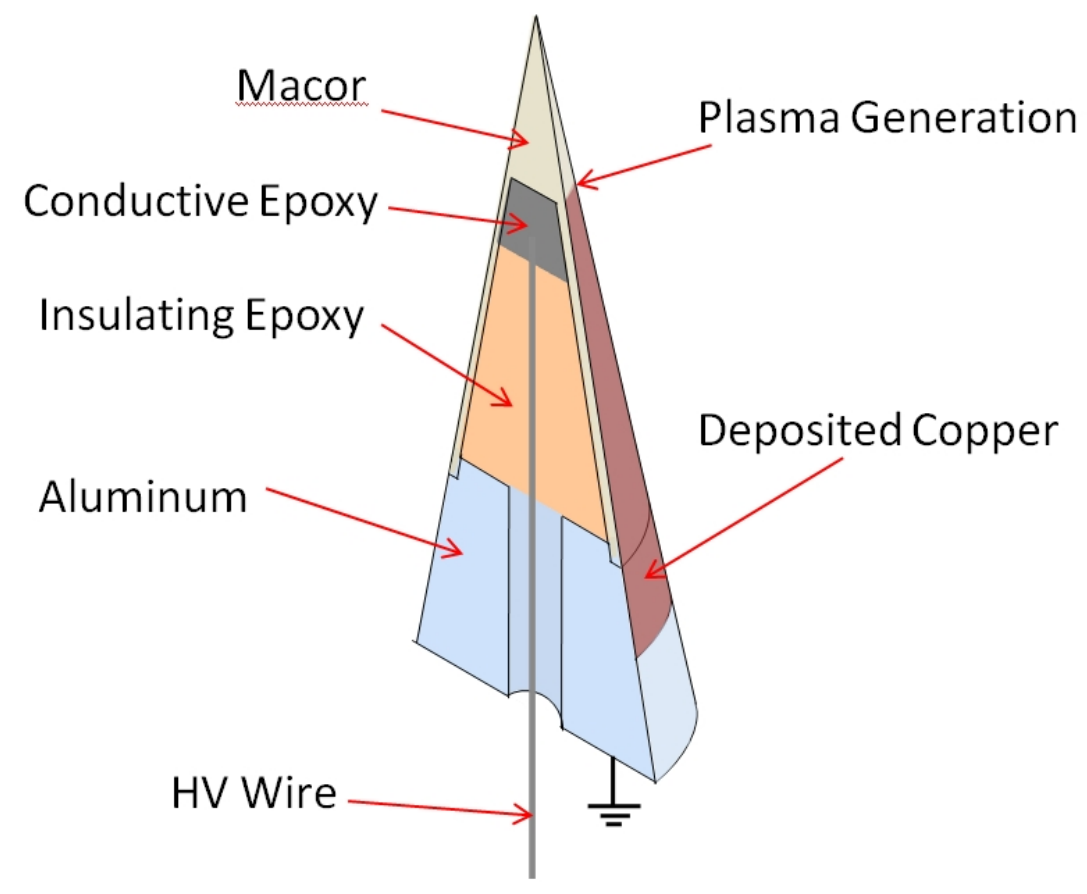

Figure 2.17. Drawing of the Macor/aluminum cone tip showing the cross section. 
over the cone tip at about an $20.3 \mathrm{~cm}$ (8 in) distance. The photo-resist was then baked in an oven for about 10 minutes at $322^{\circ} \mathrm{K}\left(120^{\circ} \mathrm{F}\right)$ to evaporate the solvent in the resist layer. The manufacturer originally recommended a higher baking temperature of about $378^{\circ} \mathrm{K}\left(220^{\circ} \mathrm{F}\right)$ on a hot plate. This however exceeded the Loctite 608 Epoxy glass transition temperature, which made the epoxy expand strong enough to push the bonded parts apart.

A mask pattern as shown on the right hand side of Figure 2.14, was designed in AutoCAD and printed with a line resolution of $10 \mu \mathrm{m}$ on a $0.1 \mathrm{~mm}$ thick polyester based transparent film.

The mask was flexible enough to be bent around the cone surface. In order to push the mask close enough onto the surface for contact masking, two conical rings made of Torlon, were slipped over the mask on the cone tip. A Spectronics ${ }^{15}$ SB-100P UV-A lamp with $4.8 \mathrm{~mW} / \mathrm{cm}^{2}$ (at $38.1 \mathrm{~cm}$ (15in) distance) was aimed at the masked cone tip, which was mounted in a mechanical rotating indexer. Sufficient exposure was achieved when the color of the reflected light from the photoresist changed from purple to an orange tone. This happened usually after 20 - 25 seconds if the light source was about $10.2 \mathrm{~cm}$ (4 in) away from the surface. The cone was then axially rotated by a quarter turn to continue the exposure.

After developing the photo-resist, a positive pattern stayed on the surface of the exposed electrode. $\mathrm{Cu}$ 49-1 copper Etchant ${ }^{16}$ was used to remove the uncoated copper, and only the desired electrode pattern remained on the Macor surface. Figure 2.18 shows the dot pattern corresponding to an azimuthal wavenumber of $m=45$.

The cone tip with the serrated edge comb structure is shown in Figure 2.19.

\footnotetext{
${ }^{15}$ Spectronics Corp., Westbury, NY 11590

${ }^{16}$ Transene Company, Inc., Danvers, MA 01923
} 


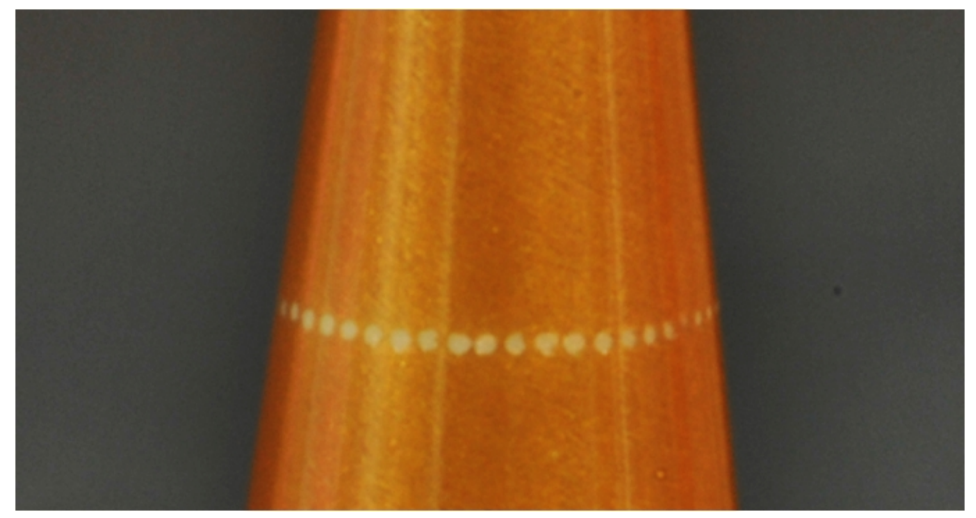

Figure 2.18. $127 \mu \mathrm{m}$ (5 mil) diameter Plasma Dots with $m=45$ spacing on a Macor dielectric cone tip

For this, there are three images: (a) the overall view of the Macor cone tip, (b) a magnified view of the electrode edge and (c) a high resolution image of separate fingers of the comb electrode. The highly magnified image of the exposed electrode pattern was taken after the wind tunnel runs with plasma were finished. It shows that the electrodes were not physically altered by the presence of the plasma.

The $m=45$ and $m=68$ comb actuators with plasma, are shown in Figure 2.20 at a $10.2 \mathrm{kPa}(3 \mathrm{inHg})$ ambient pressure. It is clearly visible that concentrations of plasma are formed at the locations of the sharp tips of the comb electrodes. Additionally there was a 2-D plasma sheet that was generated around the electrode edge. The 2-D sheet was not expected to be of consequence to either the mean flow or the cross-flow instability. The flow field generated by the actuator arrangement is further discussed in Chapter 3 . 


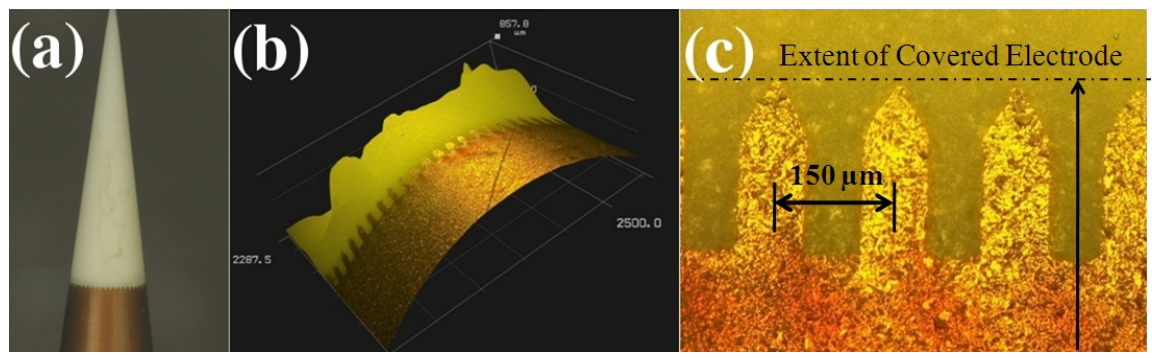

Figure 2.19. (a) shows the $m=45$ serrated edge design on the Macor cone tip. (b) is the magnified view of a $m=68$ plasma roughness tip with a Keyence Model VHX-600 3-D Imaging Microscope, and (c) is a high resolution optical image of four single "fingers" of image (b). Photograph (b) and (c) credit: Stephen Wilkinson (NASA LaRC)

\subsubsection{Surface Roughness Characterization of the Cone Tips}

The surface roughness of the cone tips was measured with a Zygo ${ }^{17}$ NewView 600 optical interferometer. Measurements on the Macor cone tip showed that the rms surface roughness was less than $0.5 \mu \mathrm{m}$. The evaporated electrode thickness was therefore less than $1 / 10^{\text {th }}$ of the rms-surface roughness, which should make it indistinguishable from the surface roughness of the Macor cone tip.

\footnotetext{
${ }^{17}$ Zygo Corporation, Middlefield, CT 06455-1291
} 

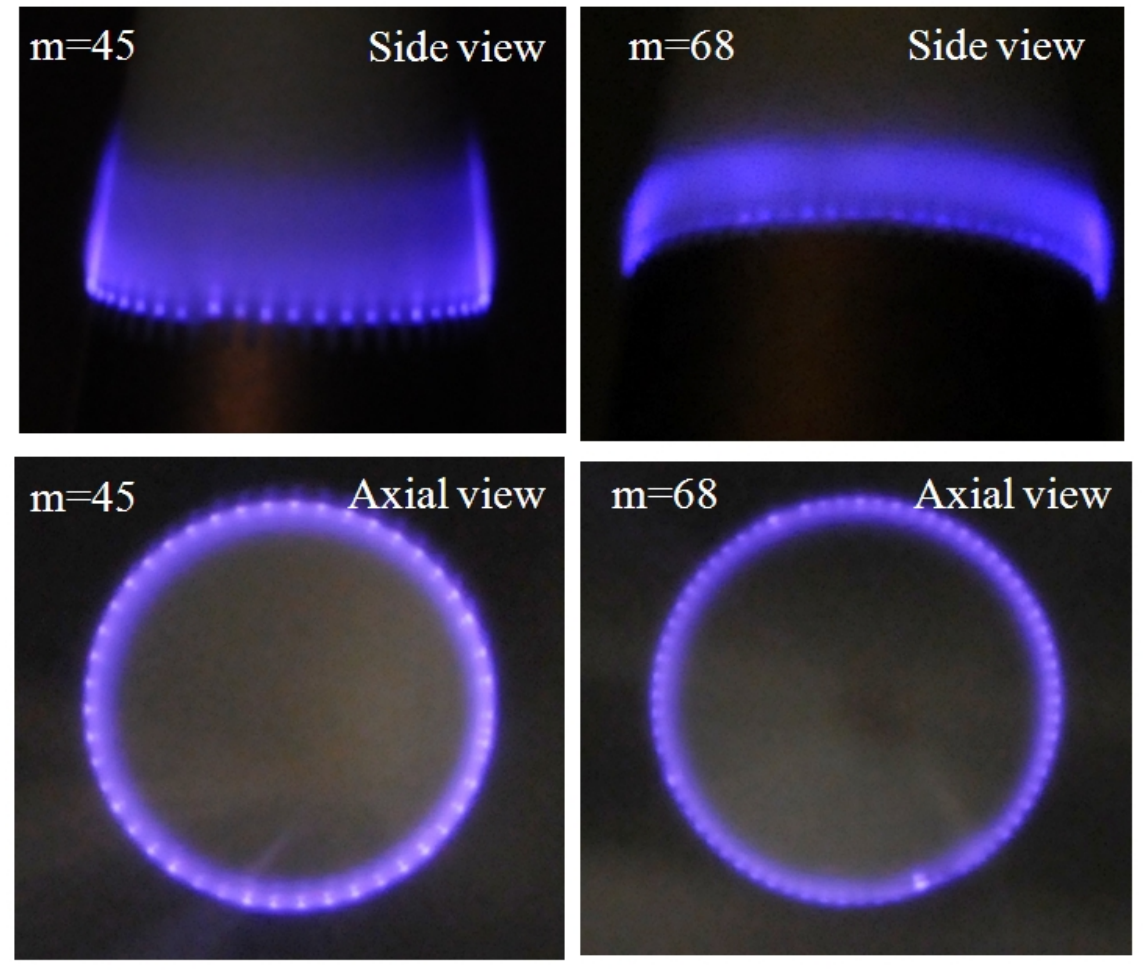

Figure 2.20. Time averaged images show the plasma pattern that forms on the Macor cone tip. $5 \mathrm{kVpp}, 10.2 \mathrm{kPa}(3 \mathrm{inHg})$ ambient pressure and $5 \mathrm{kHz}$ sine waves are applied. Concentrations of plasma are formed around the tips of the "fingers" of the serrated edge. Additionally a two dimensional plasma sheet can be seen. 


\section{CHAPTER 3}

\section{MICRO DBD PLASMA ROUGHNESS ARRAY EVALUATION}

\subsection{Circular Plasma Dots}

The circular plasma dot design on the $12.7 \mu \mathrm{m}$ (0.5 mil) thick Kapton film operated well at sub-atmospheric pressures, around $10.2 \mathrm{kPa}(3 \mathrm{inHg})$. A voltage of less than $2 \mathrm{kVpp}$ was necessary to form plasma inside the circular holes of the exposed electrode. However, the same electrode pattern on the cone tip made of the thicker Macor did not show any plasma forming up to the maximum tested voltage of $9 \mathrm{kVpp}$. It was not possible to further increase the voltage because the internal insulation between the powered and grounded electrodes was not sufficient enough to withstand higher voltages in the low pressure environment. This made it impossible to use the circular pattern for actual wind tunnel testing with the Macor cone tip.

The central difference between the two designs was the thickness of the dielectric. Although the diameter of the circular holes in the exposed electrode was increased from $76.2 \mu \mathrm{m}$ (0.003 in) to $127 \mu \mathrm{m}$ (0.005 in) for the Macor cone tip, the dielectric thickness was increased by a factor of 40 from about $12.7 \mu \mathrm{m}(0.5 \mathrm{mil})$ to $0.38-0.51 \mathrm{~mm}(15-20 \mathrm{mil})$. It is worth noting that the plasma initiation voltage for this thicker Macor, with a straight edge actuator design, was approximately $3 \mathrm{kVpp}$ at the $10.2 \mathrm{kPa}(3 \mathrm{inHg})$ pressure. 

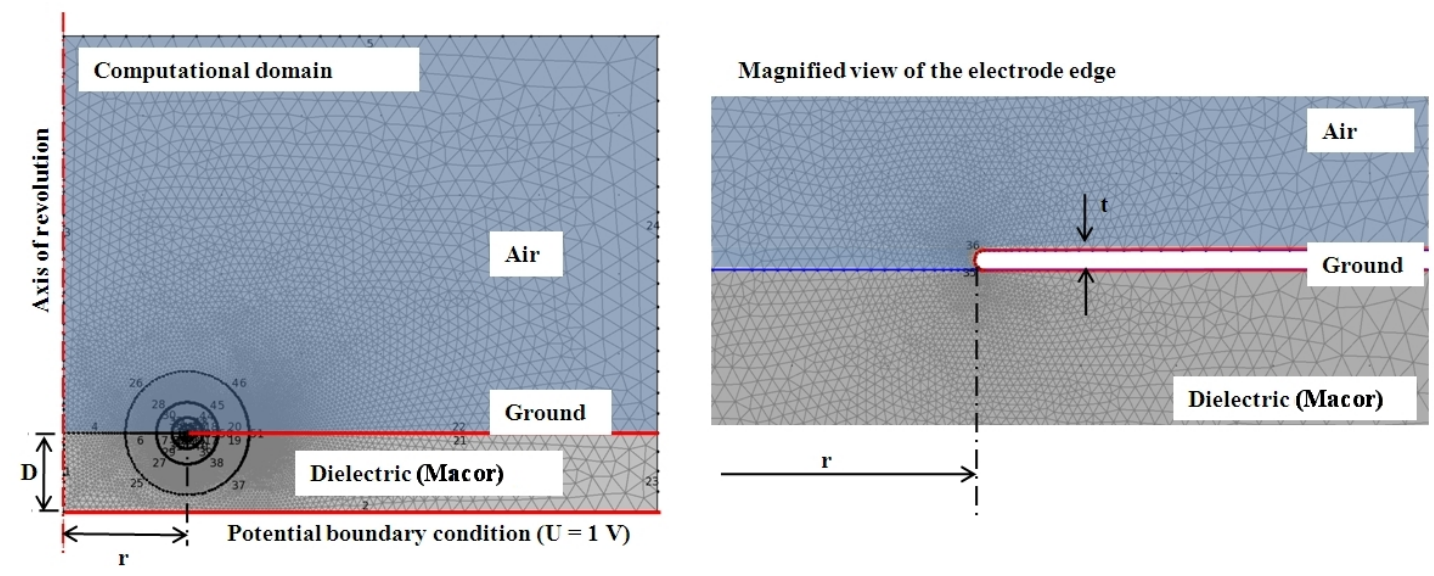

Figure 3.1. Modeling of the circular plasma actuator in Comsol. D = dielectric thickness, $\mathrm{r}=$ radius of the circular dot and $\mathrm{t}=$ thickness of the electrode. $\frac{t}{D}$ stays constant and is $4 \times 10^{-4}$. Dielectric Constant $\mathrm{k}=6$ for Macor.

In order to answer the question of why there was such a discrepancy between the two actuator designs, the electrical field magnitude in the vicinity of the electrode edges was compared. The straight-edge actuator was used as the reference case for the voltage that was necessary to cause the air next to the exposed edge to ionize.

A simulation of the electric field was conducted for a straight edge actuator. The static electrical field was calculated by solving the Poisson equation of electrostatics in $\mathrm{Comsol}^{1}$ 4.0. The geometrical model was a single circular plasma actuator like the plasma dot described in the previous chapter. The 2 D cross-section, including the computational mesh, is shown in Figure 3.1. The computational mesh consisted of 275,000 free triangular elements. This was designated as mesh $\mathrm{A}$. Mesh $\mathrm{A}$ had its highest resolution near the edge of the

\footnotetext{
${ }^{1}$ Comsol, Inc
} 
exposed grounded electrode in order to resolve the electric field gradient. Each of the concentric circles around the electrode edge had 560 nodes. The left hand side boundary of the computational domain was defined with an axisymmetric boundary condition. The bottom side of the domain was defined with an electric potential boundary condition. The remaining boundaries were defined with a charge conservation condition. The edges of the exposed electrode were set to the electric ground potential. To ensure grid independence, the same calculations were done on the same geometry with 370,000 elements and 120 nodes on the edge of the exposed electrode. This was designated as Mesh B. Each concentric circle around the electrode edge held 760 nodes in mesh B.

In order to evaluate the effect of the geometrical changes on the ionization voltage from a traditional straight edge arrangement to the circular dot arrangement, the ratio $r / D$ was introduced where $r$ is the radius of the circular dot, and $D$ is the dielectric thickness for the dot actuator. $r / D$ was about 0.15 for the $0.51 \mathrm{~mm}$ (20 mil) thick Macor dielectric and about 1.5 for the $12.7 \mu \mathrm{m}(0.5 \mathrm{mil})$ thick Kapton dielectric. Figure 3.2 shows the equipotential lines of the voltage for three $r / D$ ratios of $0.56,1.56$, and infinity.

When the radius of the hole of the exposed electrode was small, the largest electric field values were inside the dielectric material, and not in the air where ionization would occur. Only when $r / D$ was larger than 1.5 , would the electric field magnitude extend above the exposed electrode.

The distribution of the normalized electric field as a function of the distance from the edge of the exposed electrode is shown in Figure 3.3. The distance is normalized by the thickness of the dielectric. The normalized electric field is observed to increase with an increasing $r / D$ ratio. Assuming that the electric field 
Potential $U / U_{0}$ for three different circular plasma actuator geometries $\mathbf{r} / \mathbf{D}$

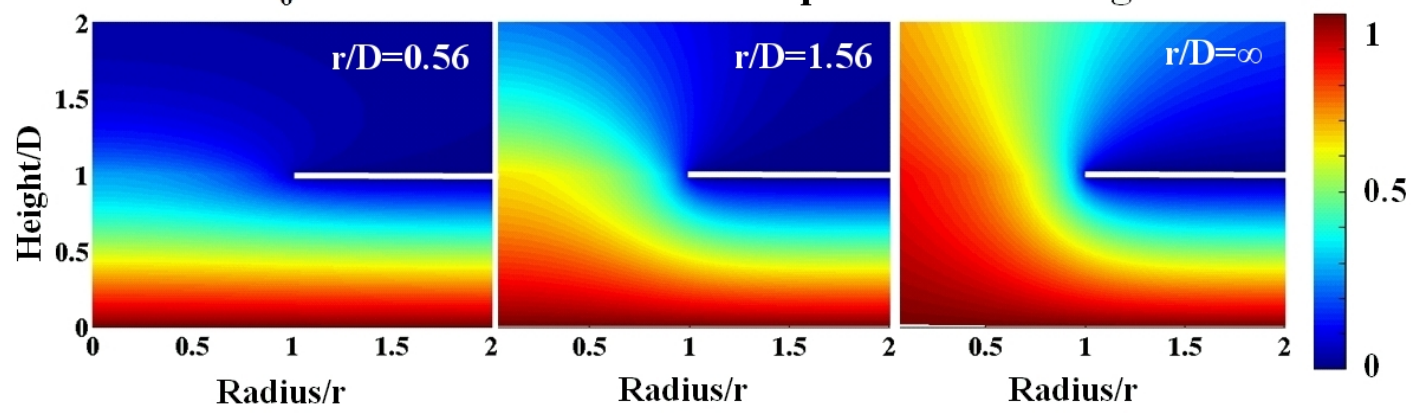

Figure 3.2. Surfaces of the normalized potential $U / U_{0}$ for $\mathrm{r} / \mathrm{D}=0.56$, $\mathrm{r} / \mathrm{D}=1.56$ and the standard straight edge arrangement $(r \rightarrow \infty)$ for mesh A.

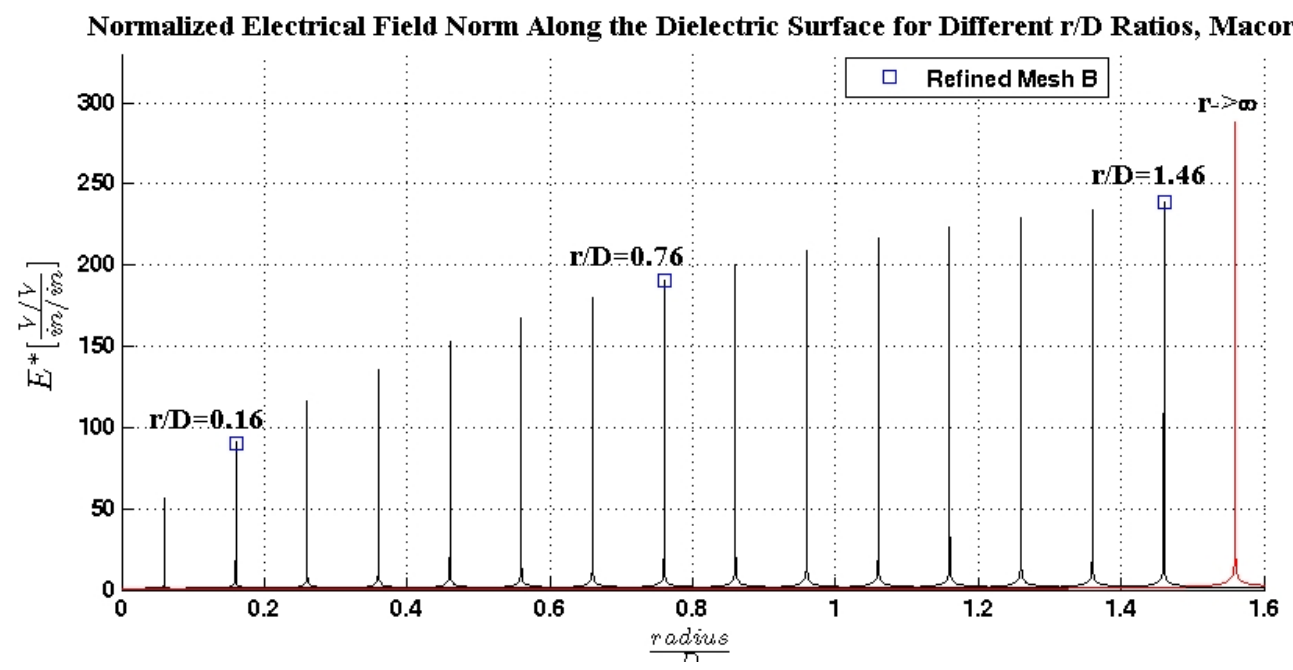

Figure 3.3. Comparison of the electrical field $E^{*}$ with the unit $\frac{V / V}{i n / i n}$ along the dielectric surface for mesh A. 
strength that was needed to ionize air at an ambient pressure of $10.2 \mathrm{kPa}(3 \mathrm{inHg})$ is the value for an infinite $r / D$, then the required normalized electric field is approximately 300. This indicates that for the electrode-to-hole ratio of 0.15 that was examined, the applied voltage needed to be at least three times larger to reach the electric field strength that was necessary to ionize the air.

This simulation provides a scaling approach for minimizing the size of a plasma actuator relative to the dielectric thickness. It indicates that the thickness of the dielectric must be commensurately reduced as the spacing between the electrodes is reduced.

\subsection{Serrated edge actuator}

The serrated edge actuator is a slightly modified miniature version of a standard plasma streamwise vortex generator [12. In order to measure the induced flow field, 2-D PIV measurements were performed in two planes around the serrated edge geometry. A $25.4 \times 25.4 \times 25.4 \mathrm{~cm}^{3}\left(10 \times 10 \times 10 \mathrm{in}^{3}\right)$ large Acrylic box was used to contain the seeding particles ${ }^{2}$ and the plasma actuator. In the first approach, a serrated edge actuator was fabricated on a flat piece of $0.38 \mathrm{~mm}$ (15 mil) thick Macor. The electrodes were $60 \mathrm{~nm}$ thick Copper that was deposited on the Macor surface. The scale of the electrodes was the same as with the $m=45$ cone tip. Unfortunately, there was difficulty in ensuring that there was sufficient particle seeding density for the PIV near the electrode. As a result, the size of the actuator was increased by a factor of eight. The dielectric was still Macor and it had a thickness of $3.25 \mathrm{~mm}$ (0.128 in). The exposed electrode in this case were fabricated from Copper tape. The Copper tape had a thickness of less than

\footnotetext{
${ }^{2} \mathrm{C}_{26} \mathrm{H}_{50} \mathrm{O}_{4}$ Bis(2-ethylhexyl)sebacate, technical grade, $90 \%$ by Sigma-Aldrich Inc., P.O. Box 14508, St. Louis, MO 63178
} 


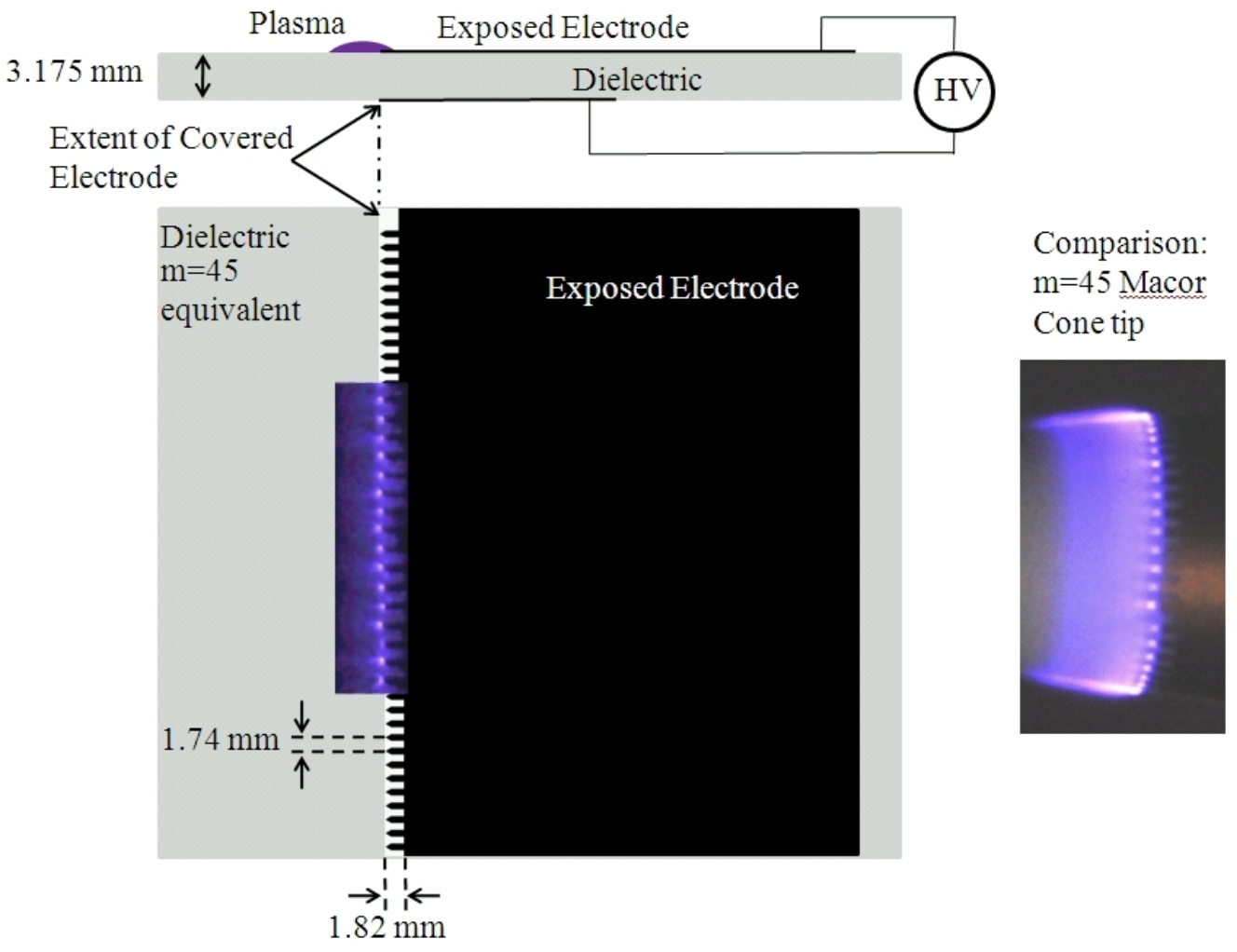

Figure 3.4. Schematic drawing of the $8 \mathrm{x}$ magnified $m=45$ equivalent serrated edge actuator with plasma-on at atmospheric pressure, $24 \mathrm{kVpp}$ and $5 \mathrm{kHz}$ sine waves. The right hand side shows a photograph of the $m=45$ Macor cone tip, $10.2 \mathrm{kPa}(3 \mathrm{inHg})$ ambient pressure, $5 \mathrm{kVpp}$, $5 \mathrm{kHz}$ sine waves 


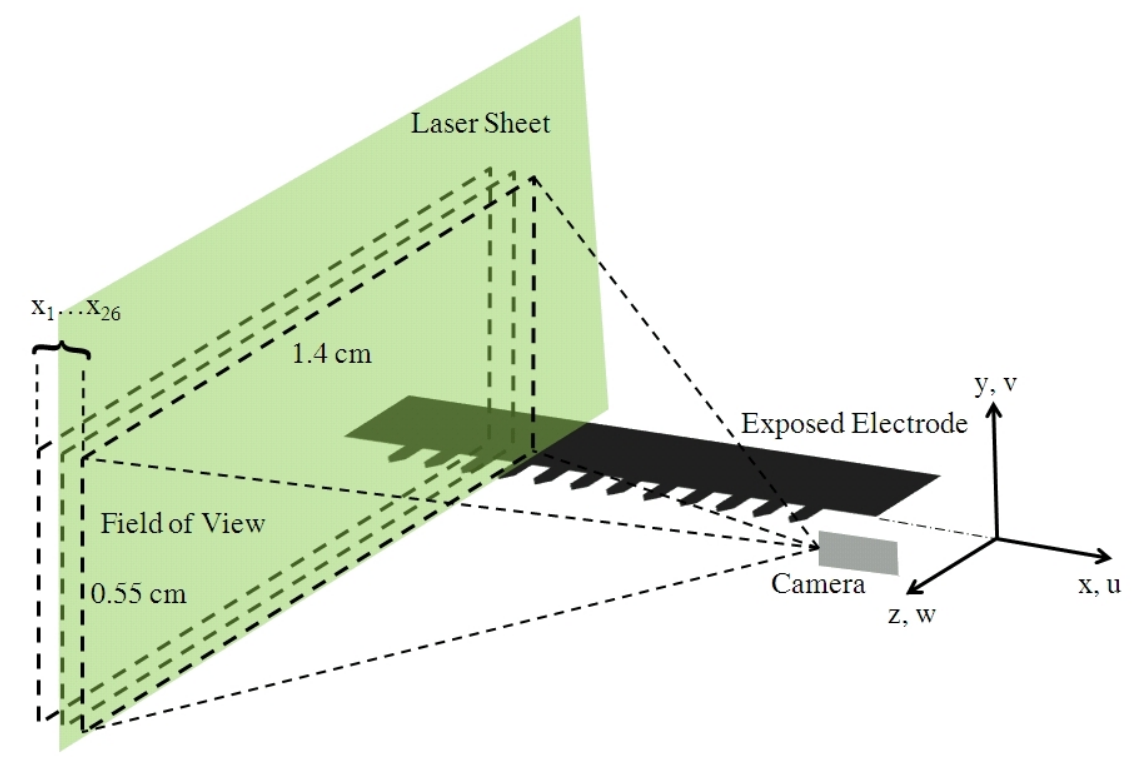

Figure 3.5. Drawing of the PIV light sheet and camera arrangement relative to the exposed serrated edge electrode

$25.4 \mu \mathrm{m}$ (1 mil). It was cut with a razor blade to the same shape as the electrode pattern used on the cone tips. The covered electrode ended at the tips of the exposed serrated electrode. This is again identical to the plasma actuators used on the cone tip.

A dimensioned drawing and photographs of the operating plasma actuator are shown in Figure 3.4. The light emission from the scaled up plasma actuator used for the PIV measurements, and the cone-tip plasma actuator are very similar. This suggests that the behavior of the induced flow field should be qualitatively similar.

The PIV system consisted of a pair of New Wave ${ }^{3}$ Nd:YAG lasers, a PIVCAM 10-30 by TSI $^{4}$ with 1000 x 1000 pixels resolution, and a TSI synchronizer. Cali-

${ }^{3}$ Electro Scientific Industries, Inc., Portland, OR 97229

${ }^{4}$ TSI Incorporated, Shoreview, MN 55126 


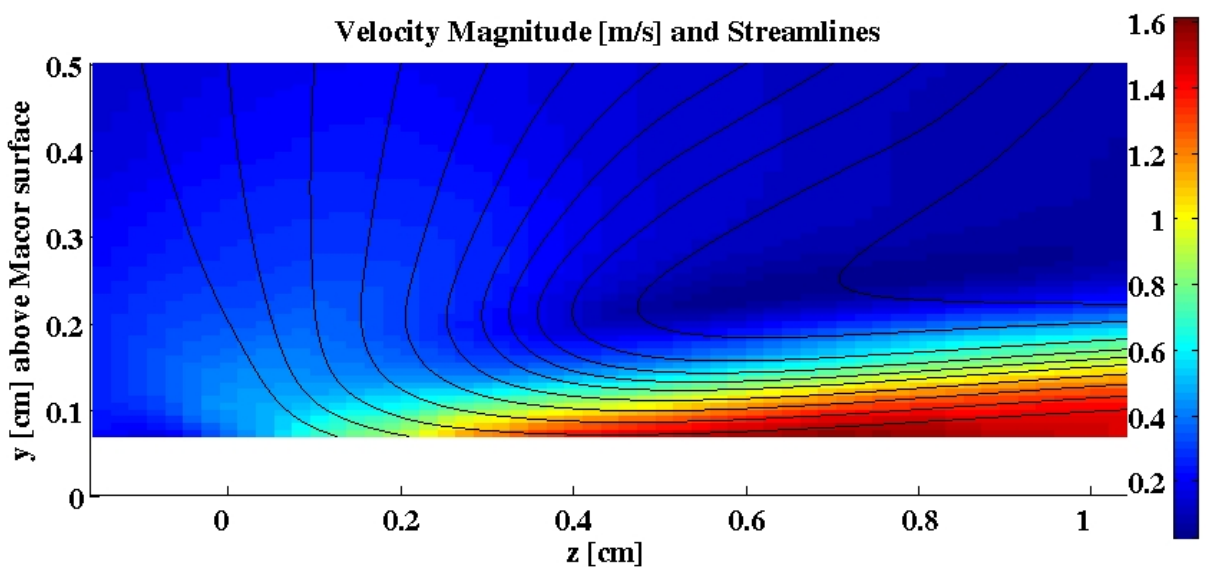

Figure 3.6. Velocity magnitude of the first plane at $\mathrm{x}=0 \mathrm{~cm}$ of $\mathrm{v}$ and $\mathrm{w}$ including streamlines.

bration was done by viewing a ruler placed in the center of the measurement field. The software accounts for any spatial distortion. For each location, 200 image pairs were taken to insure temporal convergence of the mean vector field. The interrogation area for the image correlations was set to $32 \times 32$ pixels with an overlap of 16 pixels for cross correlations. The time, $\Delta t$, between two image pairs was set so that the maximum particle displacement was roughly 10 pixels between two image exposures. Post-processing of the vector fields included a double correlation, and a filling of areas where no valid correlations occurred.

The first set of PIV images were taken in z-y planes at twenty six x-locations, as shown in Figure 3.5. The planes were spaced $0.015 \mathrm{~cm}$ apart. The x-spacing between the electrode tips of the exposed electrode was $0.174 \mathrm{~cm}$. Therefore there were approximately 12 z-y planes per spanwise wavelength of the comb electrode. The coordinate system of the measurements is indicated in Figure 3.5 .

A $5 \mathrm{kHz}$ sine wave with a voltage of $26 \mathrm{kVpp}$ was applied across the electrodes. 


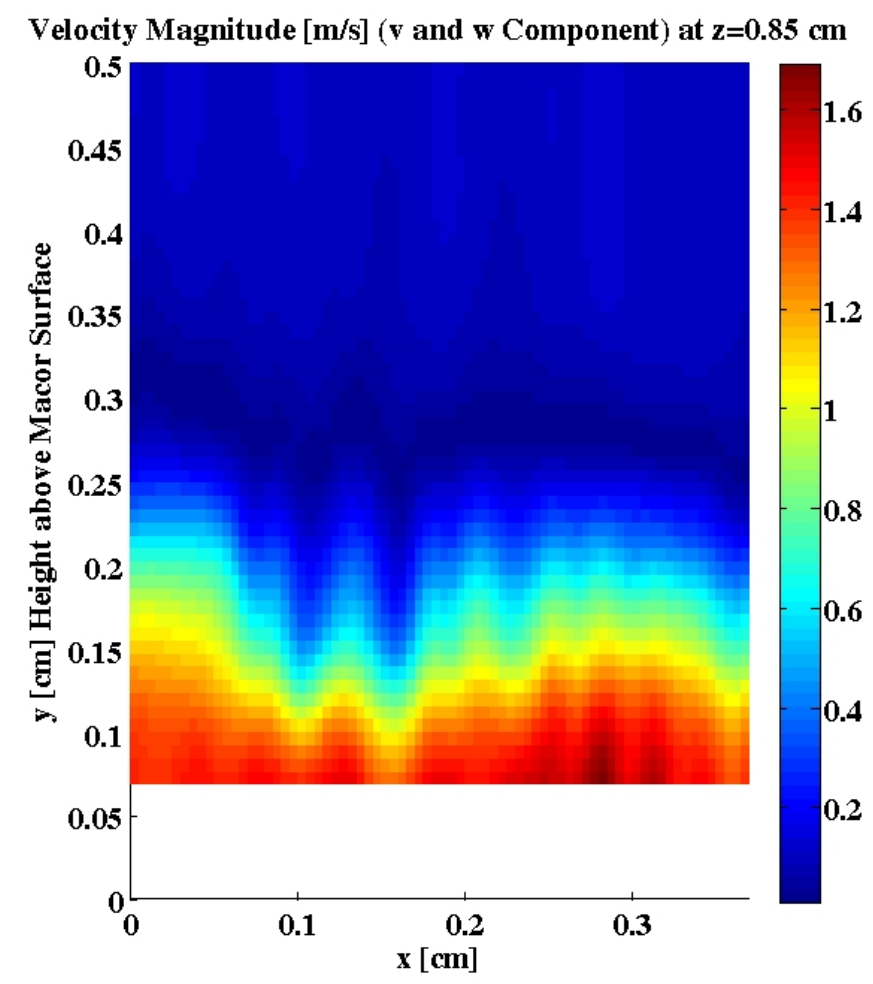

Figure 3.7. Velocity magnitude of $\mathrm{v}$ and $\mathrm{w}$ in the $\mathrm{z}$-plane at $\mathrm{z}=0.85 \mathrm{~cm}$. This plane was interpolated from a series of $26 \mathrm{x}$-planes (see Figure 3.6) starting at $\mathrm{x}=0 \mathrm{~cm}$ and ending at $\mathrm{x}=0.35 \mathrm{~cm}$

An example of the resulting velocity distribution, including stream lines, is shown in Figure 3.6 for the $z-y$ plane at $x=0 \mathrm{~cm}$. Approximately $0.075 \mathrm{~cm}$ of the field of view in the wall-normal direction were removed from the analysis due to light reflections from the copper electrode. For this, a maximum induced velocity of approximately $1.6 \mathrm{~m} / \mathrm{s}$ was measured. It can be seen that air was entrained from above the actuator edge at $\mathrm{z}=0$, and accelerated in the positive $\mathrm{z}$-direction, forming a tangential wall jet. This is what was expected from a standard straight-edge DBD plasma actuator arrangement like this.

The difference to be shown, is the variation of the flow field in the $\mathrm{x}$-direction 


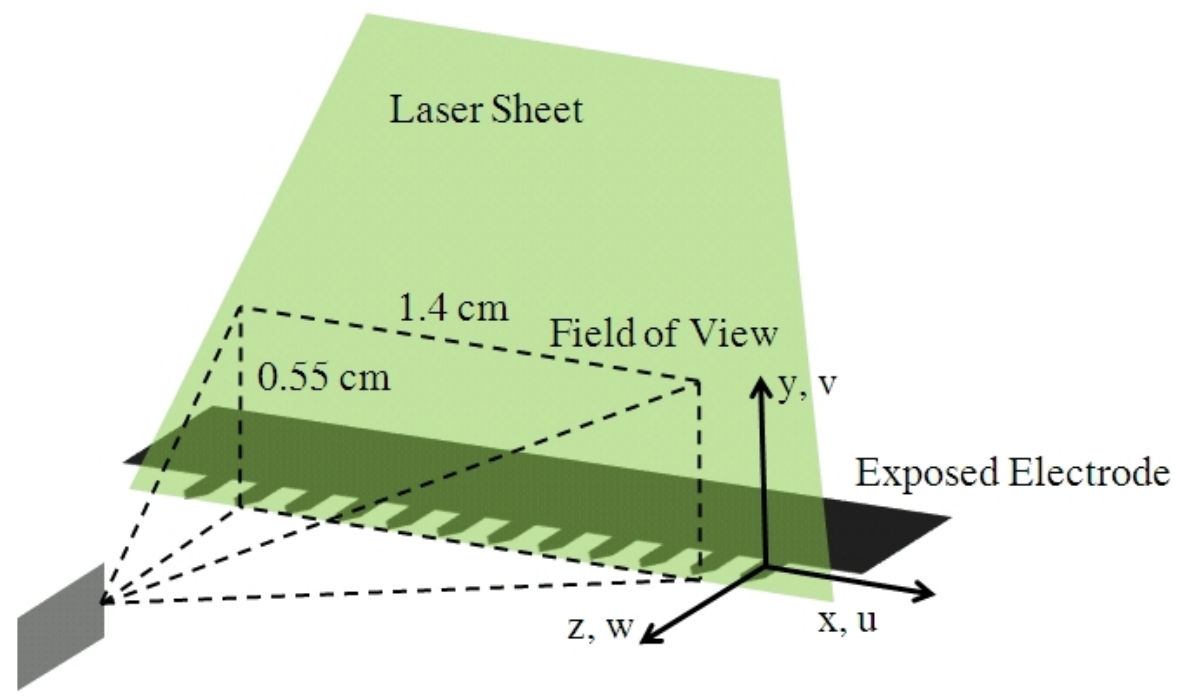

Camera

Figure 3.8. Drawing of the PIV light sheet and camera arrangement relative to the exposed serrated edge electrode

that is produced by the comb electrode arrangement. For this purpose, the 26 $\mathrm{x}$-planes were interpolated in the x-direction at constant z-locations. The result is an $\mathrm{x}-\mathrm{y}$ plane with $\mathrm{v}$ and $\mathrm{w}$ velocity components. The $\mathrm{x}-\mathrm{y}$ planes at $\mathrm{z}=0.85 \mathrm{~cm}$ is shown in Figure 3.7. This shows that the tangential wall jet observed in Figure 3.6 changes with the spanwise $\mathrm{x}$-location. The $\mathrm{x}-\mathrm{y}$ plane shows a periodic velocity distribution. The maxima are about $2 \mathrm{~mm}$ apart, which corresponds to the spanwise wavelength of the exposed comb electrode pattern.

Given this spanwise variation of the tangential velocity, vorticity along the z-direction was then examined. For this, the $\mathrm{u}$ and $\mathrm{v}$ velocity components in the $\mathrm{x}-\mathrm{y}$ plane were measured. The arrangement to measure the $\mathrm{u}$ and v velocity components is shown in Figure 3.8. The $\mathrm{x}-\mathrm{y}$ plane was located at $\mathrm{z}=0.2 \mathrm{~cm}$, which corresponds to a position slightly in front of the tips of the comb electrode. As 


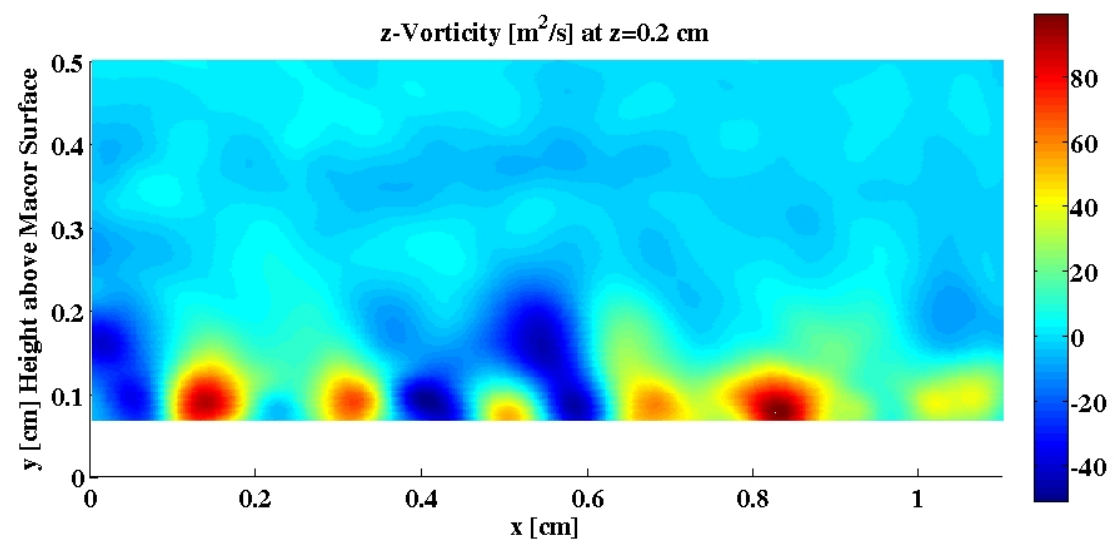

Figure 3.9. z-Vorticity of the serrated edge arrangement. The z-plane is positioned about $0.2 \mathrm{~cm}$ downstream of the actuator edge.

before, approximately $0.07 \mathrm{~cm}$ above the Macor surface was removed from the PIV image because of surface reflections.

Figure 3.9 shows the z-vorticity calculated from the measured $\mathrm{u}-\mathrm{v}$ velocity field. This shows a regular pattern of alternating signed z-vorticity, with an x-spacing of approximately $0.18 \mathrm{~cm}$. This spacing almost exactly matches the spanwise wavelength of the comb electrode.

The PIV results show that the geometry that was used for the plasma actuator patterned roughness generated streamwise vorticity at the spanwise wavelength of the comb electrodes. The mean flow distortion produced by the generated streamwise vorticity would therefore result in a spanwise periodic thickening an thinning of the boundary layer with the same wavelength as the comb electrodes.

As mentioned at the start of this chapter, the PIV measurements were performed on a comb electrode actuator that was eight times larger than the actual version on the cone tip. A question that might be asked is how the flow induced by the actuator, in quiescent air, relates to that with a non-zero free stream velocity. 
The introduction of streamwise vorticity was identified as the desired mechanism for the excitation of the stationary cross-flow vortices. Crocco's theorem with the added effect of an external body force gives some answers.

The formulation of Crocco's theorem including a body force $\vec{f}$, is shown below. Starting with the inviscid stationary Navier Stokes equation

$$
\rho \vec{V} \cdot \nabla \vec{V}=\vec{f}-\nabla p
$$

and using the vector identity

$$
\vec{V} \cdot \nabla \vec{V}=\nabla \frac{V^{2}}{2}-\vec{V} \times(\nabla \times \vec{V})
$$

one obtains the following equation

$$
\vec{V} \times(\nabla \times \vec{V})=\nabla \frac{V^{2}}{2}-\frac{\vec{f}}{\rho}+\frac{1}{\rho} \nabla p .
$$

Now $\nabla \times \vec{V}=\vec{\omega}$ is the vorticity in the flow. The specific enthalpy in the flow is

$$
d h=T d s+\frac{1}{\rho} d p
$$

or in general,

$$
T \nabla s=\nabla h-\frac{1}{\rho} \nabla p
$$

as shown in [66]. The stagnation enthalpy is defined as

$$
H=h+\frac{V^{2}}{2}
$$


and can be substituted together with Equation 3.5 and Equation 3.6 into Equation 3.3 to give the following equation 3.7 .

$$
\vec{V} \times(\nabla \times \vec{V})=-\frac{\vec{f}}{\rho}+\nabla H-T \nabla s
$$

If the right hand side of Equation 3.7 is non-zero, vorticity along the corresponding axis is generated. In our case, we are looking for the generation of streamwise vorticity. This can only be the case if there is at some point a velocity component normal to the surface. If there is a wall normal velocity component, a spanwise azimuthal body force, as well as spanwise or azimuthal gradients in the stagnation enthalpy or entropy, vorticity is added to the flow.

As long as a plasma forms, a wall-normal component of velocity at the actuator edge is undoubtedly generated regardless of the scale of the comb actuator. This is induced by electrodynamic body forces that has components in the wall-normal and tangential directions.

As seen in the photograph of the operating plasma actuator on the cone tip, concentrations of bright plasma forms at the tips of each comb electrode. The DBD process forms a non-equilibrium plasma in which the electron temperature is several orders of magnitude higher than the ion temperature, which is assumed to be equal to the total temperature of the surrounding air [13]. Therefore, an entropy or enthalpy gradient is mainly attributed to the electron temperature difference, which is large. However, due to its limited mass, it has only a small effect on the average gas temperature. The temperature gradient produced by even the most energetic plasma is therefore very small, and is most likely negligible to the generation of vorticity.

The strongest effect on the generation of vorticity for the DBD plasma actuator 
is achieved by the body force $\vec{f}$. The comb electrodes produce body force vectors that are in the azimuthal direction. Based on equation 3.7, this will produce a streamwise vorticity component in the flow. Compressibility effects would appear as a change of density and alter therefore the body force term in the presented notation. Based on Balakumar's baseline calculations [3], the normalized density $\rho / \rho_{\infty}$ is 0.4 on the leeward ray and 0.6 on the windward ray at $\mathrm{x}=0.5$ in

If the discharge became thermal such as through a breakdown of the dielectric material that leads to an arc, the generation of vorticity would be dominated by entropy and enthalpy gradients. Arc discharges have ion temperatures up to several thousand Kelvin and electron temperatures exceeding 20,000 K [6]. The main mechanism in this case is then Joule heating. However in the present experiment the DBD actuators were designed and operated so that only the body force vector field was the mechanism affecting the flow, and in this case, generating vorticity. 


\section{CHAPTER 4}

\section{RESULTS WITH PASSIVE DISTRIBUTED ROUGHNESS}

The first step of the experiments, was to verify that stationary cross-flow vortices were present in the boundary layer over the cone at an angle of attack. Following that, the receptivity of the stationary cross-flow modes to the passive roughness needed to be demonstrated. Finally, the focus was on if subcritical forcing delayed the onset of laminar-turbulent transition in the same way as it does in a subsonic flow. The following sections illustrate the results of surface flow visualization to visualize stationary cross-flow modes, and Pitot probe measurements to quantitatively evaluate the receptivity and laminar-turbulent transition of the boundary layer due to passive roughness.

\subsection{Surface Flow Visualization}

The surface flow visualization was an indirect measure of the boundary layer shear stress at the surface of the cone. The surface flow visualization fluid is removed faster from the surface in regions of locally higher shear stress. In the case of stationary cross-flow vortices, it appears as a pattern of light and dark streaks on the surface of the cone. The pattern corresponds to thinner and thicker accumulations of the surface visualization fluid that are formed by the higher shear stress directly under the stationary cross-flow vortices. 
Half of the cone surface was coated with a fluid mixture consisting of 15 parts of $1000 \mathrm{cs}$ silicon oil, 5 parts of oleic acid, and 1 part $\mathrm{TiO}_{2}$ particles. Different stagnation pressures between $172.4 \mathrm{kPa}(25 \mathrm{psia})$ and $344.7 \mathrm{kPa}(50 \mathrm{psia})$ were investigated for the three different passive roughness cone tips. Videos showing the development of the surface patterns were taken. Frames from the videos are shown in Figure 4.1. The visible part of the cone which shows the surface flow visualization, ranges from $\mathrm{x}=25.4 \mathrm{~cm}$ (10 in) to $\mathrm{x}=35.6 \mathrm{~cm}$ ( $14 \mathrm{in})$. In each video, the initially uniform oil coating developed into the representative stationary pattern. The surface visualization pattern then remained unchanged beyond that point in time. All of the images, except when otherwise stated, were run in the wind tunnel with the bleed valves open. This is the "quiet" operation mode.

In the images, the notable difference with increasing Reynolds number is that the clear pattern of streamwise streaks disappears, and is replaced by a diffuse layer of white visualization oil. The streaks are assumed to be the result of stationary cross-flow vortices. For $\mathrm{P}_{0}=172.4 \mathrm{kPa}(25 \mathrm{psi})$, these are present over the entire visible surface of the cone from an azimuthal angle of $30^{\circ}$ to $180^{\circ}$.

A comparison between the surface flow visualization for the three different roughness cone tips shows that there is a different azimuthal distribution of the cross-flow vortices with each tip. This is a first indication that the boundary layer was receptive to the different patterned roughness distributions. When the streaky pattern that dominated at $\mathrm{P}_{0}=172.4 \mathrm{kPa}(25 \mathrm{psi})$ disappeared at higher stagnation pressures, turbulent transition was assumed to have taken place. The smooth baseline tip at $\mathrm{P}_{0}=241.3 \mathrm{kPa}(35 \mathrm{psi})$ shows almost no sign of stationary cross-flow vortices, except for three or four streaks on the leeward side of the cone. If the bleed valves were closed these streaks disappeared completely. Looking at 


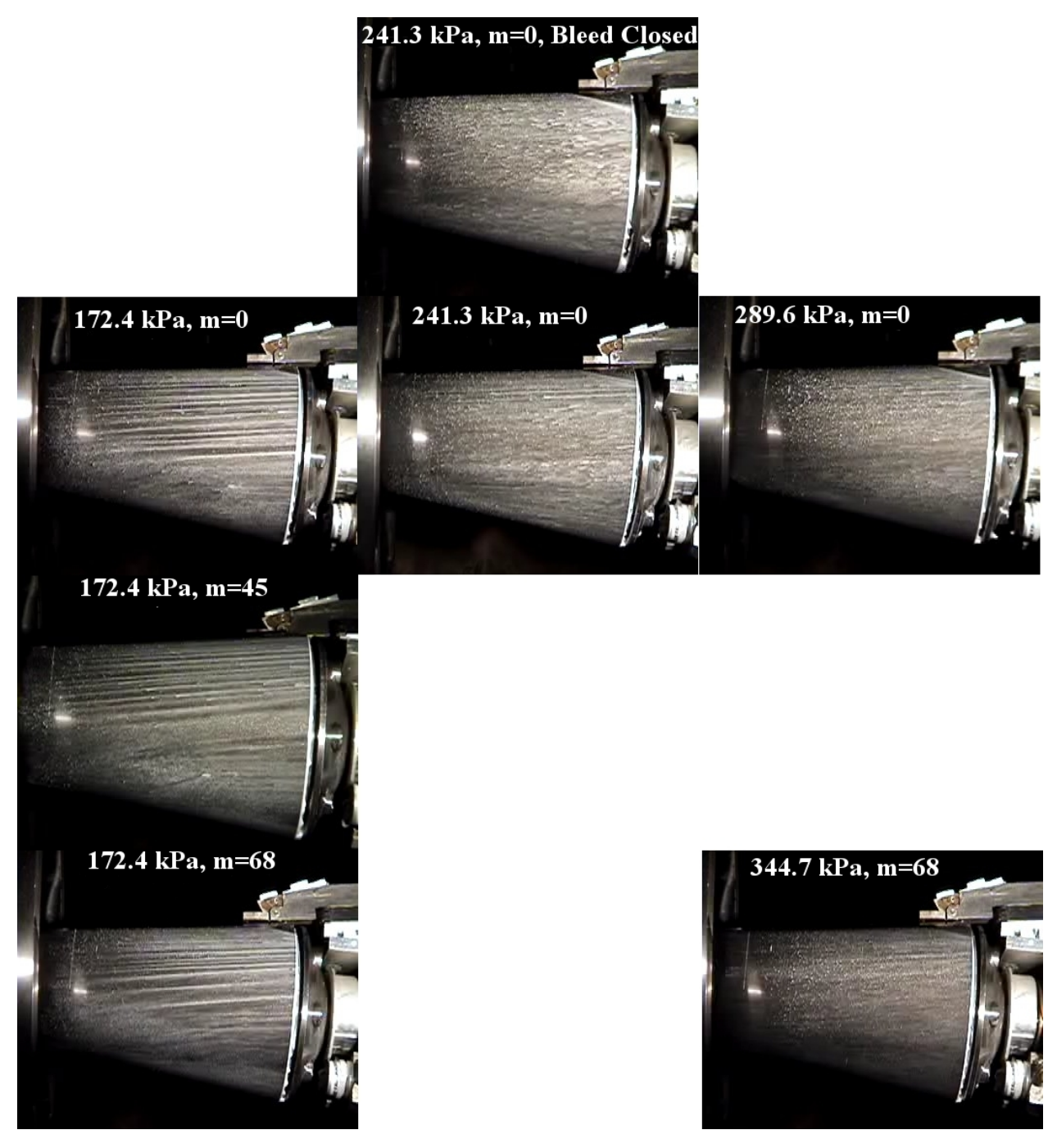

Figure 4.1. Surface flow visualization at different unit Reynolds numbers and flow conditions at Mach 3.5. $\mathrm{P}_{0}=172.4 \mathrm{kPa}(25 \mathrm{psi}) \rightarrow$

$$
\begin{gathered}
R e=9.84 \times 10^{6} \mathrm{~m}^{-1}, \mathrm{P}_{0}=241.3 \mathrm{kPa}(35 \mathrm{psi}) \rightarrow R e=1.37 \times 10^{7} \mathrm{~m}^{-1} \\
\mathrm{P}_{0}=289.6 \mathrm{kPa}(42 \mathrm{psi}) \rightarrow R e=1.65 \times 10^{7} \mathrm{~m}^{-1} \text { and } \mathrm{P}_{0}=344.7 \mathrm{kPa} \\
(50 \mathrm{psi}) \rightarrow R e=1.96 \times 10^{7} \mathrm{~m}^{-1}
\end{gathered}
$$




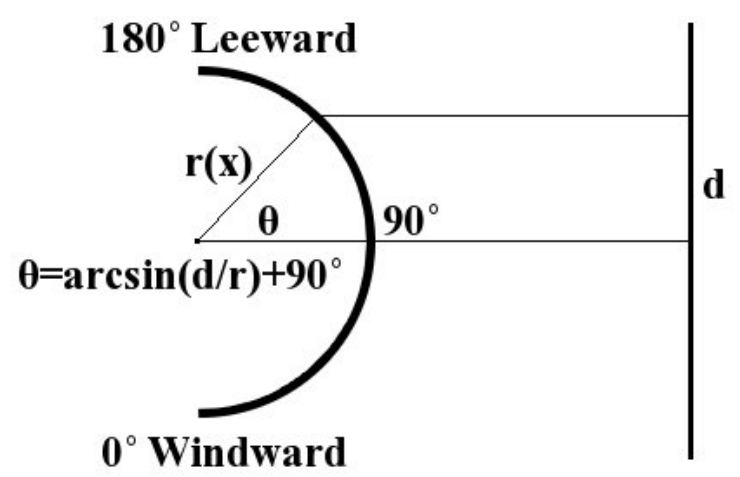

Figure 4.2. Schematic of the unwrapping of the conical surface onto the observer plane of the camera.

the flow visualization at $\mathrm{P}_{0}=289.6 \mathrm{kPa}$ (42 psi) with open bleed valves, some faint streaks are still observed on the leeward side of the cone.

With the bleed valves open, we expect that the freestream disturbances are small. As a result, transition to turbulence should be dominated by stationary cross flow modes. The surface flow visualization supports this conclusion.

Since the flow visualization pattern of parallel streaks corresponds to the presence of cross-flow vortices, their spacing can be used for a wavenumber analysis. A coordinate transformation from the flat $\mathrm{x}-\mathrm{y}$ camera plane onto the conical $x-\theta$ plane was done to obtain the azimuthal spacing from the original video images for a wavenumber analysis. A schematic of the projection method is shown in Figure 4.2. If the axial x-location is known, the radius $r$, and the azimuthal angle $\theta$ can be calculated from the observer plane coordinate $d$. Pixel coordinates are transformed into physical coordinates through known dimensions in the photograph. An example of the "unwrapped" cone surface is presented in Figure 4.3. The top of the figure is the original photograph, taken from the flow visualization video. 


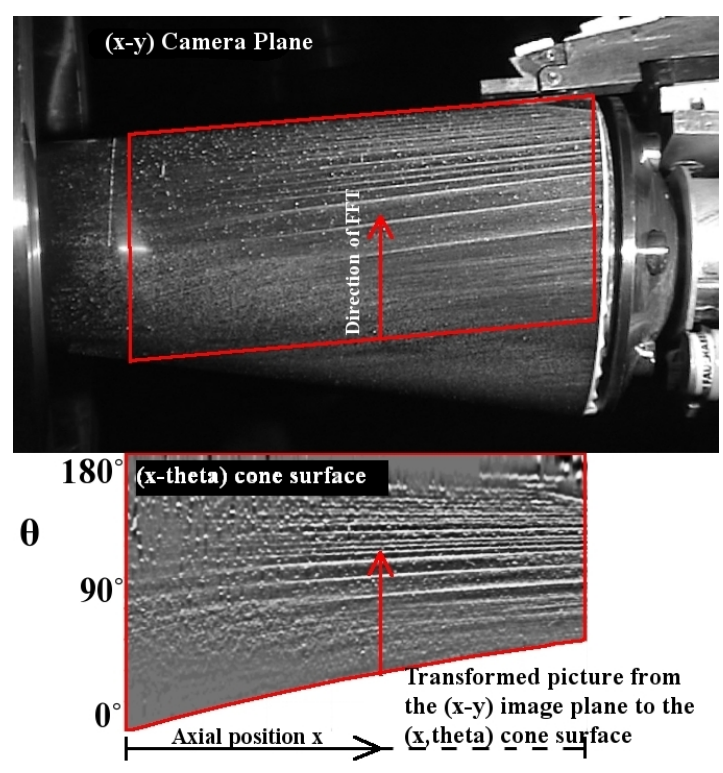

Figure 4.3. Example showing the original and the projected image used for further analysis.

The red box indicates the region that is unwrapped onto a $x-\theta$ coordinate system that is shown in the bottom of the figure.

The unwrapped image was used to calculate a wavenumber spectrum in the $\theta$-direction for each axial position. The result of the spectral analysis is shown in Figure 4.4. The figure shows the flow visualization image and the corresponding wave number spectrum for the smooth baseline case $(m=0)$, the critical baseline case $(m=45)$, and the subcritical case $(m=68)$, at $\mathrm{P}_{0}=172.4 \mathrm{kPa}(25 \mathrm{psi})$. The spectral amplitudes have been normalized by their respective maximum values. The amplitudes range from 0 , corresponding to the blue color to 1 which corresponds to the red color. In the smooth (baseline) tip, most of the energy is at wavenumbers between $m=50$ and 60 . This agrees well with the linear theory predictions. The smooth tip case also shows a second band of energy at higher wavenumbers between $m=100$ and 130 . These might be due to higher harmonics 


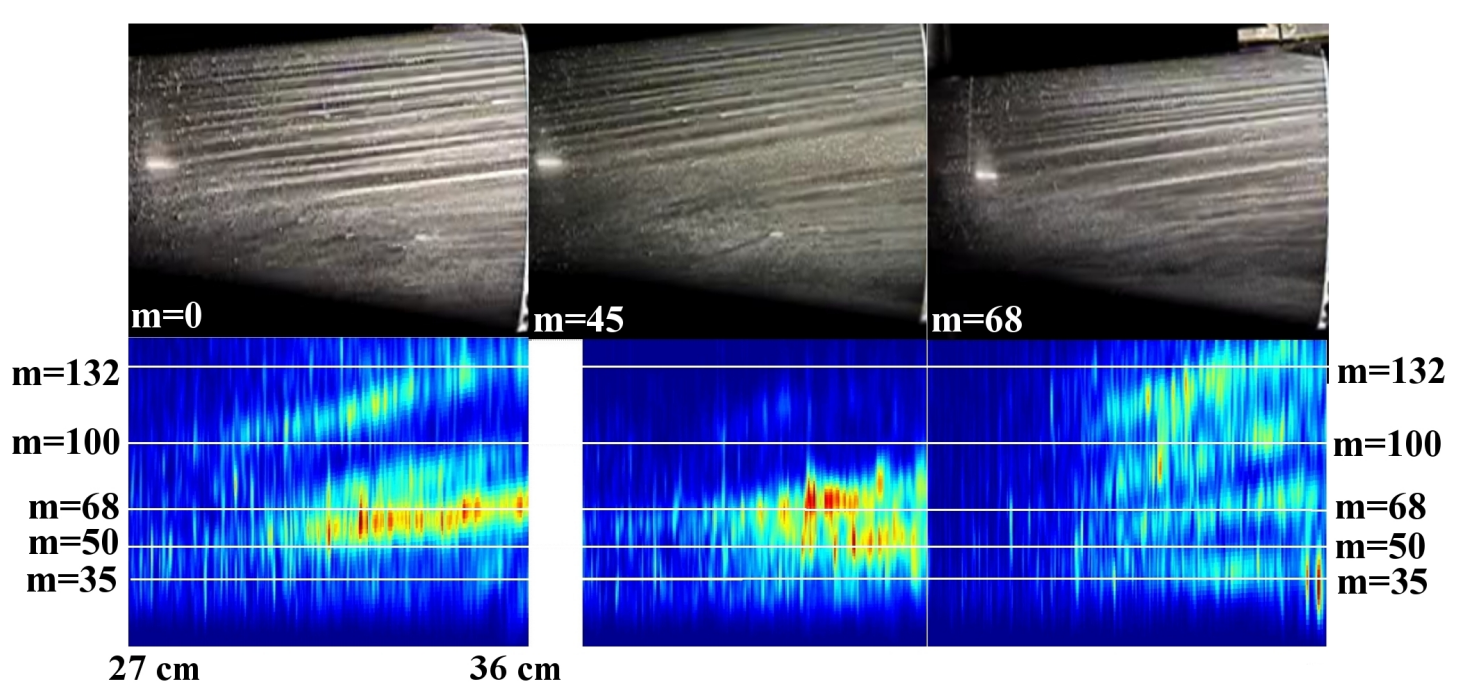

Figure 4.4. Surface flow visualization and wavenumber spectra for the smooth baseline case, the critical wavenumber baseline case, and the subcritical case at different axial locations.

of the stationary cross-flow mode. Similar higher harmonics have been observed by Saric et al. 45 .

The $m=45$ roughness tip represents the controlled critical forcing case. The wave number spectra in this case indicate that the critical forcing at $m=45$ produced energy at that wave number. In addition, it suppressed the energy at the higher harmonic wave number that were present with the smooth tip.

The wave number spectra for the $m=68$ roughness indicates that the subcritical forcing put energy into $m=68$. In contrast to the critical forcing, energy at higher wave numbers was also present, somewhat like with the smooth tip. It is clear that the subcritical forcing reduced the energy in the linearly most amplified band of wave numbers between $m=50-60$. In addition there was some energy at lower wave numbers near $m=40$ that arose from the $m=68$ roughness. 


\subsection{Pitot Tube Surveys}

The Pitot probe surveys made it possible to directly measure the mean flow distortion in the boundary layer and therefore the azimuthal wave numbers of the stationary cross-flow modes. This was measured by taking azimuthal profiles at a constant height above the cone surface, inside the boundary layer. The Pitot probe measured an increase or decrease in the total pressure, that was a direct indication of the thickening and thinning of the boundary layer.

\subsubsection{Receptivity to Forcing Wavenumber}

The azimuthal variation of the total pressure in the boundary layer for the smooth $(m=0)$ tip is shown in Figure 4.5. For this, the axial location of the Pitot probe was at $\mathrm{x}=28.45 \mathrm{~cm}(11.2 \mathrm{in})$, which at $\mathrm{P}_{0}=172.4 \mathrm{kPa}(25 \mathrm{psi})$ is at $R e_{x}=2.79 \times 10^{6}$.

The measured azimuthal total pressure distribution was analyzed in three ways. First, the data series was high-pass filtered at a wavenumber of $m=25$ to remove long wavelength variations. An example of the high-pass filtered data series is shown in the top part of Figure 4.5. A Fast Fourier Transform was performed on the data series to identify the wavenumber content. The result is shown in the middle part of Figure 4.5. It can be seen that the majority of the energy is contained near a wavenumber of $m=55$.

Finally, a wavelet analysis of the filtered data series was performed. For this, Morlet wavelets were used as shape functions. The wavelet analysis identified the azimuthal locations where different wavenumbers were present. This indicated that $m=55$ was present at azimuthal angles from $110^{\circ}$ to $180^{\circ}$.

The filtered azimuthal pressure distribution for the $m=68$ roughness case at 


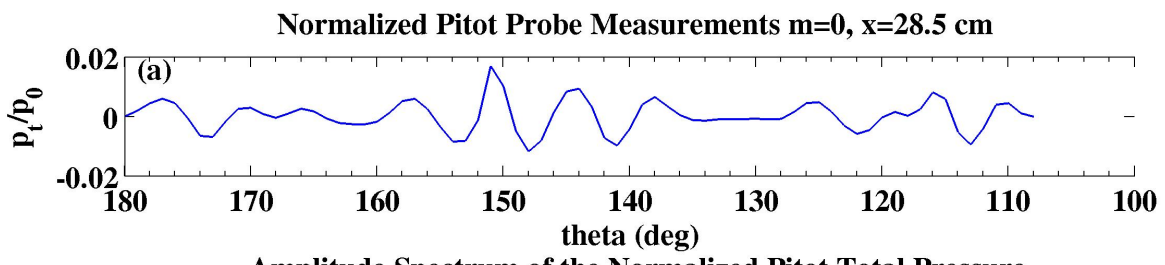

Amplitude Spectrum of the Normalized Pitot Total Pressure
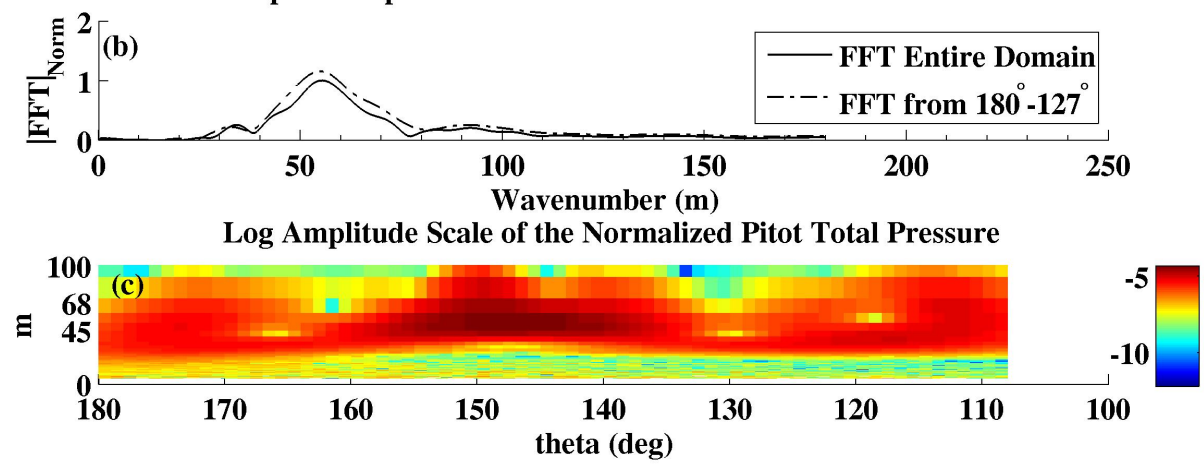

Figure 4.5. Pitot probe azimuthal scans (a), amplitude spectra (b), and wavelets (c) at $38 \mu \mathrm{m}(0.0015 \mathrm{in})$ above the surface and $\mathrm{x}=28.45 \mathrm{~cm}$ (11.2 in), $m=0$ cone tip, $\mathrm{P}_{0}=172.4 \mathrm{kPa}(25 \mathrm{psi})$.

$\mathrm{x}=28.45 \mathrm{~cm}(11.2 \mathrm{in})$ is shown in the top of Figure 4.6. The spectral analysis of the data series is shown in the middle part of the Figure. Although it shows that the azimuthal wave number with the largest energy is at $m=50$, there is also a peak at $m=68$. The wavelet analysis at the bottom part of the figure is quite different from the smooth tip case. In particular, energy at $m=68$ is more dominant between $130^{\circ}<\theta<180^{\circ}$. When the spectral analysis was focused on that azimuthal range, it showed the largest peak at $m=68$.

The filtered azimuthal pressure distribution for the $m=45$ case at $\mathrm{x}=28.45 \mathrm{~cm}$ (11.2 in) is shown in the top part of Figure 4.7. The wave number spectra for this case is shown in the middle part of the figure. Considering the whole azimuthal domain, the dominant peak is near $m=45$. The bottom part of the figure shows 


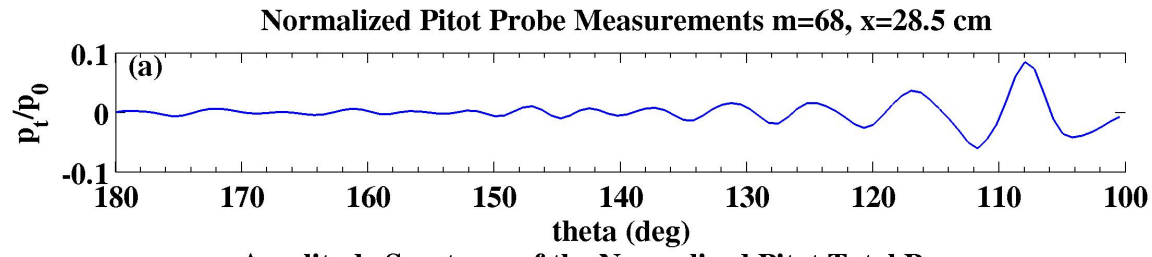

Amplitude Spectrum of the Normalized Pitot Total Pressure

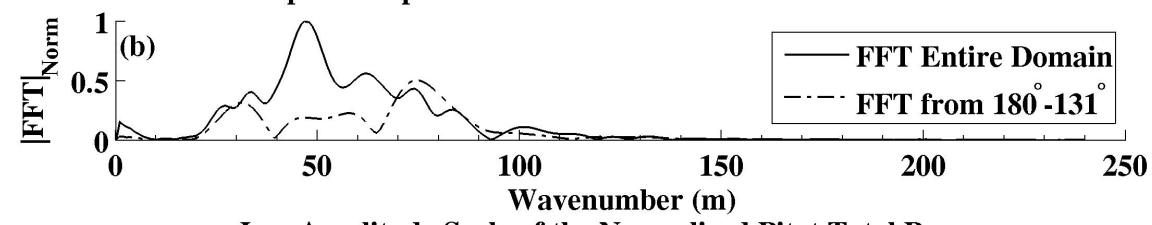

Log Amplitude Scale of the Normalized Pitot Total Pressure

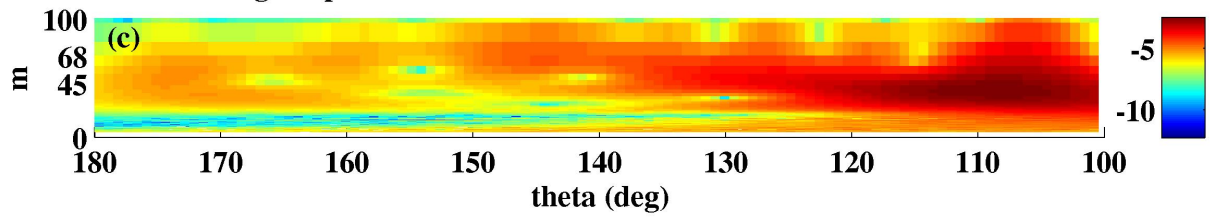

Figure 4.6. Pitot Probe azimuthal scans (a), amplitude spectra (b), and wavelets (c) at $38 \mu \mathrm{m}(0.0015$ in) above the surface and $\mathrm{x}=28.45 \mathrm{~cm}$ (11.2 in) $m=68$ cone tip, $\mathrm{P}_{0}=172.4 \mathrm{kPa}(25 \mathrm{psi})$. 


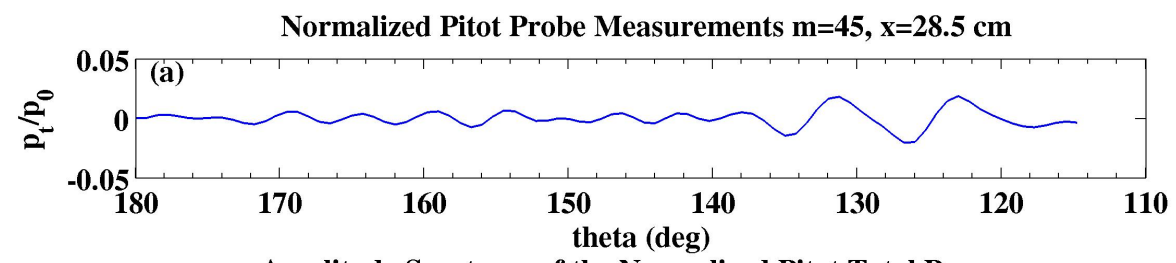

Amplitude Spectrum of the Normalized Pitot Total Pressure

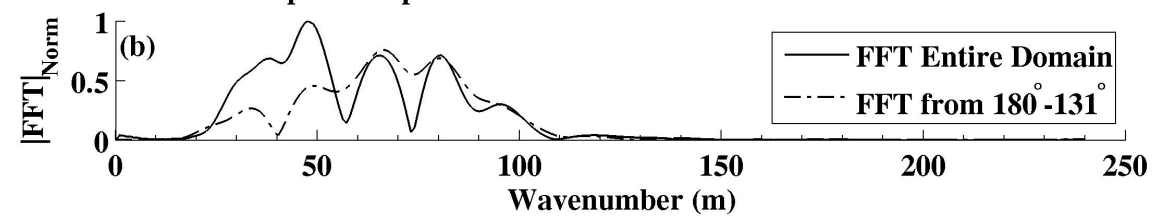

Log Amplitude Scale of the Normalized Pitot Total Pressure

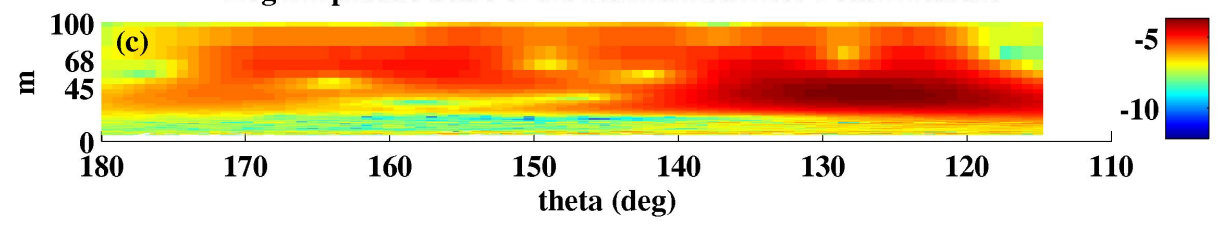

Figure 4.7. Pitot probe azimuthal scans (a), amplitude spectra (b), and wavelets (c) at $38 \mu \mathrm{m}(0.0015$ in) above the surface and $\mathrm{x}=28.45 \mathrm{~cm}$

(11.2 in) with the $m=45$ cone tip, $\mathrm{P}_{0}=172.4 \mathrm{kPa}(25 \mathrm{psi})$.

the wavelet analysis. This appears more similar to that for the smooth tip, and is quite different from the $m=68$ case. In particular is the dominant $m=45$ at all azimuthal angles.

Additional azimuthal pressure series were performed at $\mathrm{x}=25.9 \mathrm{~cm}$ (10.2 in) which at $\mathrm{P}_{0}=172.4 \mathrm{kPa}$ was $R e_{x}=2.55 \times 10^{6}$. Figure 4.8 shows the result for the $m=68$ roughness cone tip. When the whole azimuthal domain was considered, the dominant wave number was at $m=50$. However, using the wavelet results as a guide, when the azimuthal range was between $130^{\circ}<\theta<180^{\circ}$, the dominant wavenumber was at $m=68$ of the the patterned roughness.

The azimuthal pressure distribution for the $m=45$ case at the same location of $\mathrm{x}=25.9 \mathrm{~cm}(10.2 \mathrm{in})$ is shown in Figure 4.9 . In this case, as at $x=11.2 \mathrm{in}$ in 

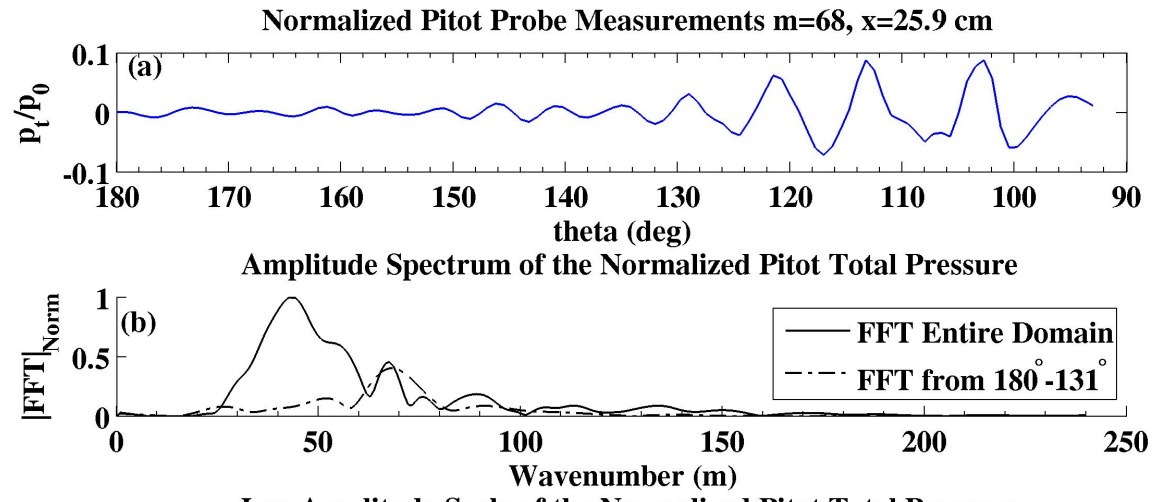

Log Amplitude Scale of the Normalized Pitot Total Pressure

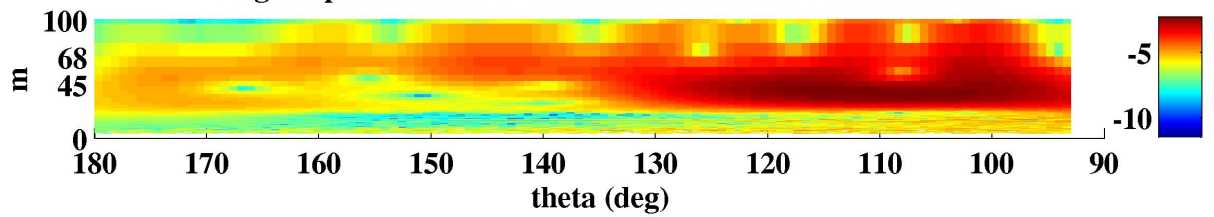

Figure 4.8. Pitot probe azimuthal scans (a), amplitude spectra (b), and wavelets (c) at $38 \mu \mathrm{m}(0.0015 \mathrm{in})$ above the surface and $\mathrm{x}=25.9 \mathrm{~cm}$ (10.2 in), $m=68$ cone tip, $\mathrm{P}_{0}=172.4 \mathrm{kPa}(25 \mathrm{psi})$. 


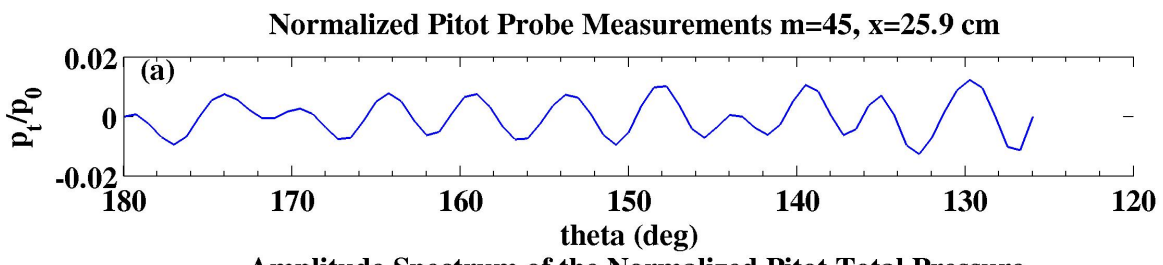

Amplitude Spectrum of the Normalized Pitot Total Pressure

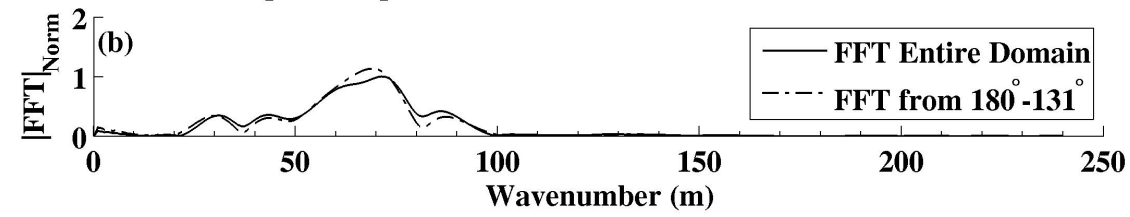

Log Amplitude Scale of the Normalized Pitot Total Pressure

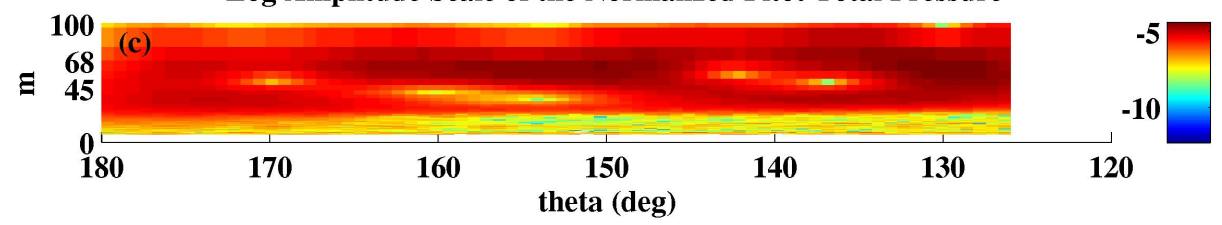

Figure 4.9. Pitot probe azimuthal scans (a), amplitude spectra (b), and wavelets (c) at $38 \mu \mathrm{m}(0.0015 \mathrm{in})$ above the surface and $\mathrm{x}=25.9 \mathrm{~cm}$ (10.2 in), $m=45$ cone tip, $\mathrm{P}_{0}=172.4 \mathrm{kPa}(25 \mathrm{psi})$.

Figure 4.7, the dominant wave number is independent of the azimuthal extent. The dominant wave number is near $m=79$, but a smaller peak occurs at $m=45$.

The results with the three cone tips clearly indicate that the boundary layer was receptive to both the $m=45$ and the $m=68$ patterned roughness. The linear stability analysis predicted that the most amplified stationary cross-flow mode had a wavenumber near $m=45$. This was consistent with the smooth tip as well as the $m=45$ roughness tip which appeared to reduce the range of higher wave numbers that appeared in the spectrum. The azimuthal range over which the $m=68$ appeared was further towards the leeward side of the cone compared to the $m=45$ or the smooth tip. The reason for this will be explained later in the results. 


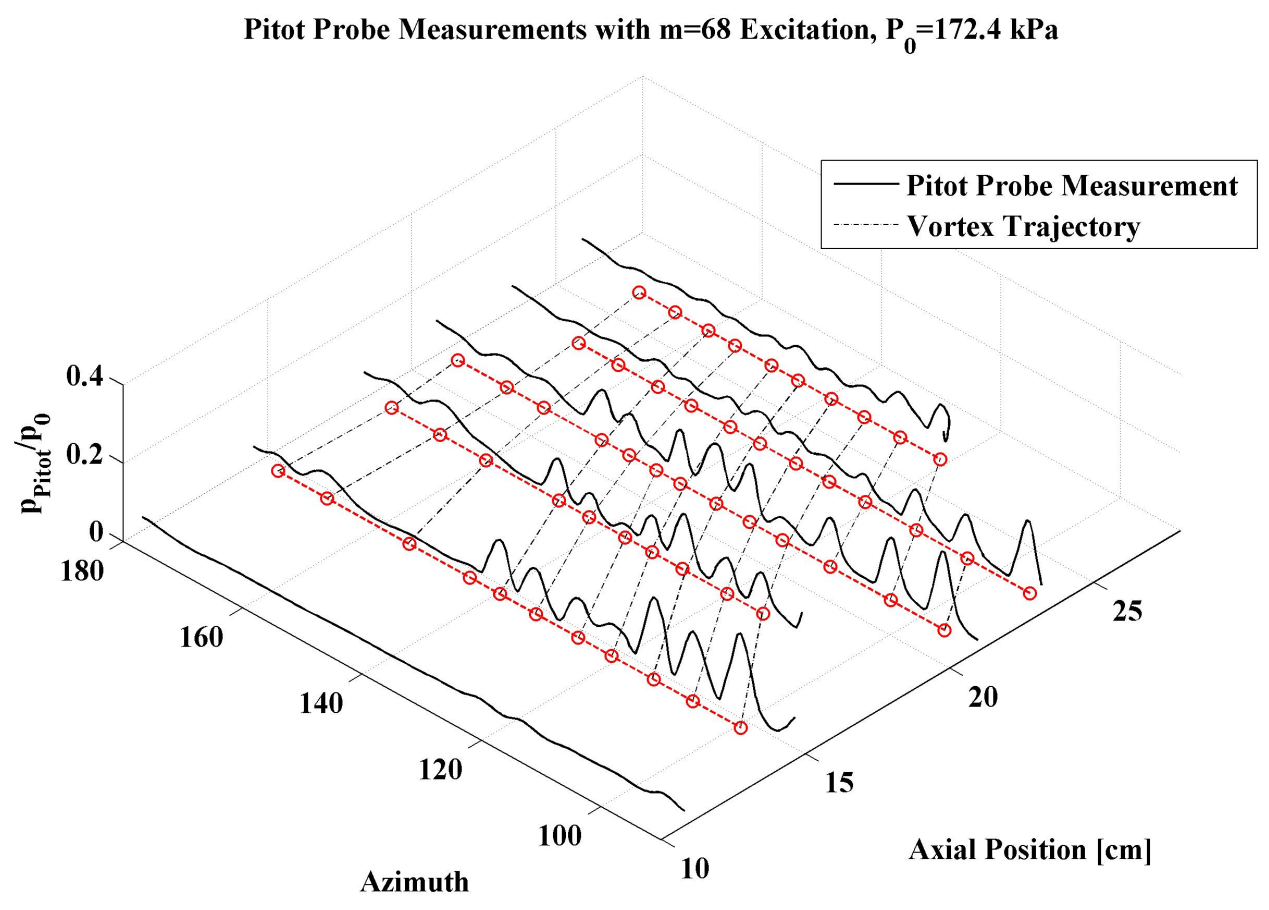

Figure 4.10. Pitot tube scans at different axial and azimuthal locations at $38 \mu \mathrm{m}(0.0015 \mathrm{in})$ above the surface for $m=68$.

\subsubsection{Cross Flow Vortex Path}

The paths of the stationary cross-flow vortices were identified by connecting the pressure maxima of azimuthal pressure data series at different axial locations. The result for the $m=68$ roughness tip is shown in Figure 4.10. The trajectories are observed to curve towards the leeward side of the cone. The amplitudes appear larger towards the wind ward side of the cone, but in that region the boundary layer is thinner so that the mean flow distortion is more pronounced.

The cross-flow mode trajectories in Figure 4.10 were overlaid onto a plot of the mean streamlines of the baseline flow in Figure 4.11. Also included in the figure are the predicted paths of $m=40$ stationary cross-flow vortices based on 
Balakumar's DNS simulation [3]. The top part of the figure represents the data in $\mathrm{x}-\theta$ coordinates wheres the bottom part shows the same data, projected on the physical surface of the cone. We observe that even though they are at different wave numbers, the trajectory of the measured and simulated cross-flow vortex paths agree pretty well. This suggests that the simulated vortex paths could be used to predict the paths of the measured cross-flow vortices into regions where no measurements were taken. This is especially interesting for extrapolating in the upstream direction towards the location of the roughness array at $\mathrm{x}=1.27 \mathrm{~cm}$ (0.5 in). Based on this, we note that most of the paths of cross-flow vortices originated near the windward side of the cone. This is not surprising since the stationary cross-flow modes are expected to follow the mean flow streamlines, and they originate on the windwards side of the cone.

In order to get an estimate of the azimuthal location where the surface roughness could affect the growth of the stationary cross-flow modes, the limiting or dividing vortex path line was estimated. For this the focus was on the $m=68$ roughness case. Recall in the previous section that the $m=68$ was only dominant over a smaller range of azimuthal angles $180^{\circ}<\theta<130^{\circ}$. Therefore the crossflow vortex path where the roughness-induced wavenumber transitioned away from $m=68$ is identified as the azimuthal location of the parting vortex path.

The location of the parting vortex was examined using the wavelet analysis that is shown in the bottom part of Figure 4.12. This was at a far upstream location of $\mathrm{x}=12.7 \mathrm{~cm}(5 \mathrm{in})$. The wavelet transform indicated that the azimuthal region where the energy at $m=68$ disappeared, started around $\theta=115^{\circ}$. Based on this, an azimuthal region of ineffective forcing of roughness at $\mathrm{x}=12.7 \mathrm{~cm}$ ( $5 \mathrm{in}$ ) was applied to Figure 4.11 as shaded blue portion of the plot. This region was 
Comparison of DNS Streamlines and Vortex Trajectories with

Measured Vortex Trajectories

(a) $\mathrm{x}$-theta plane
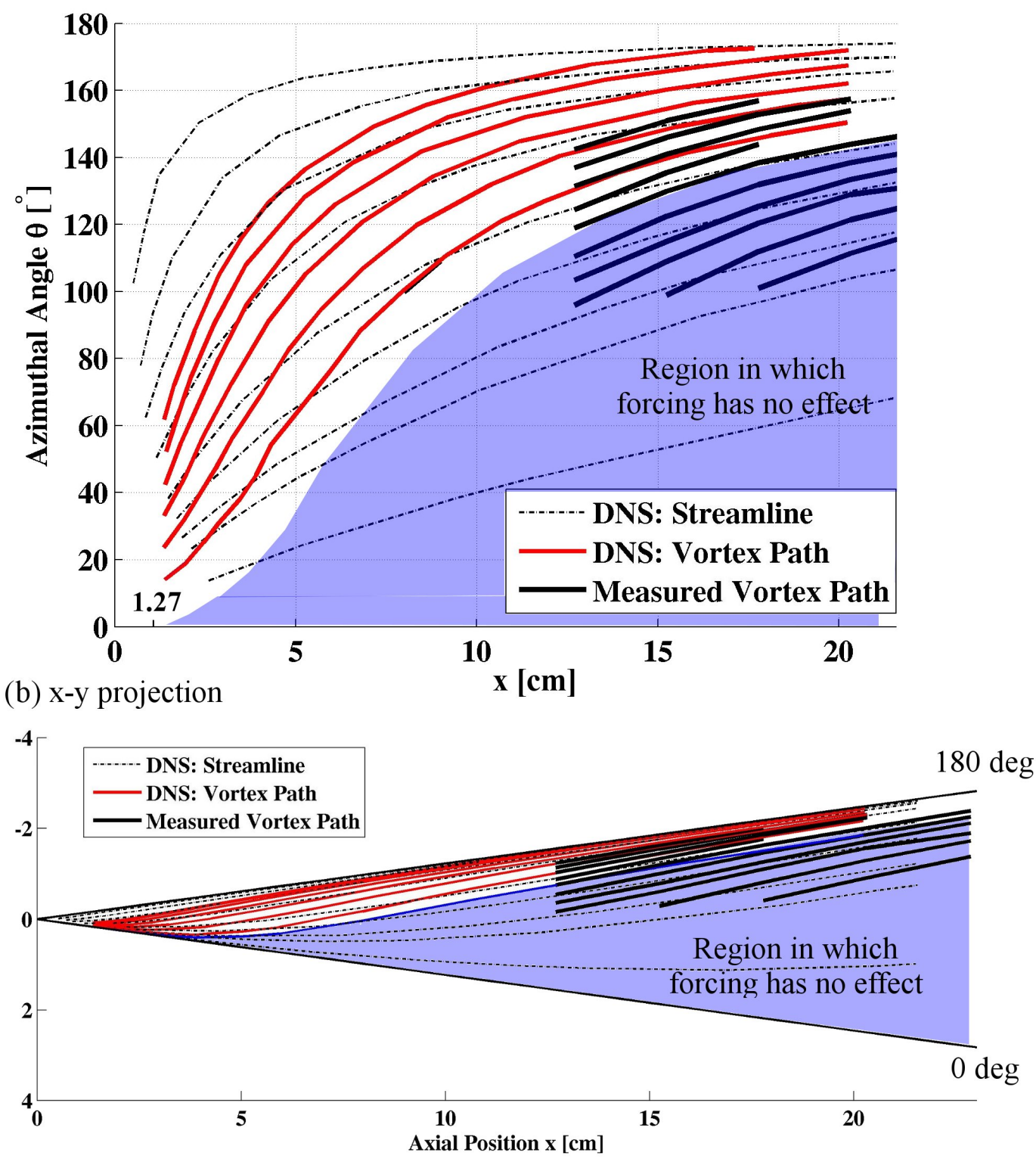

Figure 4.11. Cross-flow vortex paths with the $m=68$ roughness tip in comparison with the surface streamlines of the baseline flow and vortex paths of Balakumar's DNS calculations [3] with a $m=40$ distributed disturbance at $\mathrm{x}=1.27 \mathrm{~cm}(0.5 \mathrm{in})$. (a) shows the $\mathrm{x}-\theta$ plane and (b) corresponds to the projection on the observer $\mathrm{x}-\mathrm{y}$ plane. 

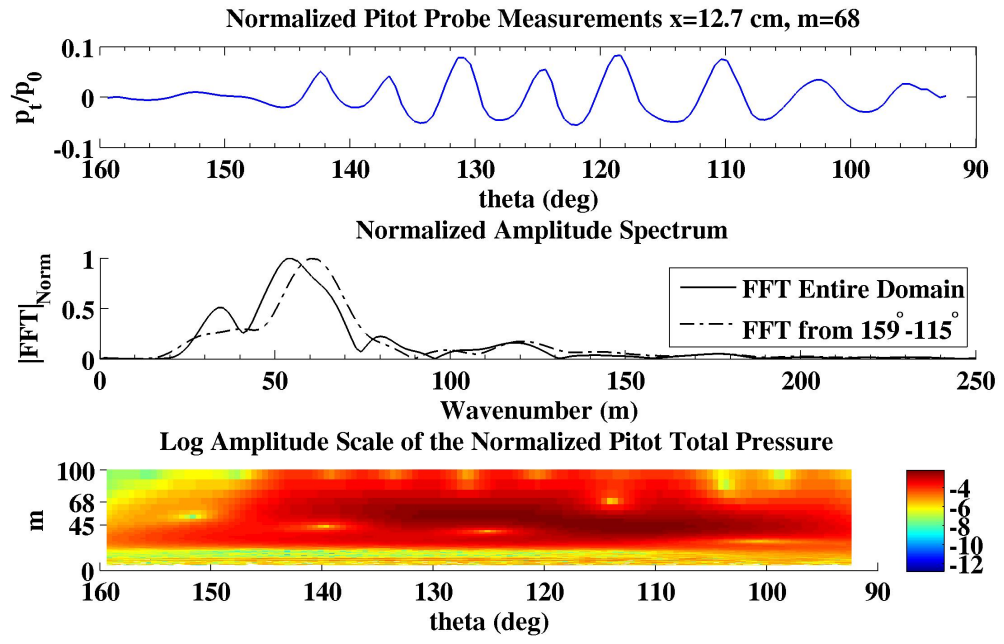

Figure 4.12. $m=68$ roughness tip, azimuthal scan at $\mathrm{x}=12.7 \mathrm{~cm}(5 \mathrm{in})$, $0.05 \mathrm{~mm}$ (0.002 in) Pitot probe height. Wavenumber spectrum (center) and Wavelet analysis (bottom). $\mathrm{P}_{0}=172.4 \mathrm{kPa}(25 \mathrm{psi})$

estimated by adding an azimuthal offset to the most windward stationary crossflow vortex path from the DNS simulation until it overlayed the measured parting vortex path. The addition of the azimuthal offset had to be done since there was no vortex trajectory present for the experimentally identified parting vortex trajectory in Balakumar's DNS simulation. Interestingly, the offset DNS-simulated vortex trajectory points towards an azimuthal angle of $0^{\circ}$ close to the roughness location at $\mathrm{x}=1.27 \mathrm{~cm}(0.5 \mathrm{in})$. This is a very strong evidence that the parting vortex line had its origin at the very windward point of the roughness array $\left(\theta=0^{\circ}\right)$ at $\mathrm{x}=1.27 \mathrm{~cm}(0.5 \mathrm{in})$ on the cone tip. 


\subsubsection{Detailed Wavenumber Analysis}

Figure 4.11 indicates that the paths of the measured stationary cross-flow vortices have a certain inclination angle relative to the rays of constant azimuth. This inclination angle is not constant for each axial location, which means that if the wavenumber of the stationary vortices is estimated by moving around the circumference at a fixed axial location, the wavenumber of the cross-flow mode changes with each axial location. This effect is especially significant closer to the tip of the cone where the vortex paths are highly inclined relative to the constant azimuth rays.

The change in the azimuthal wavenumber with increasing Reynolds number from $1.25 \times 10^{6}$ to $1.75 \times 10^{6}$ is shown in Figure 4.13 . The range of the analyzed azimuthal angles is based on the location of the previously identified parting vortex path. Each azimuthal pressure data series was additionally padded with zeros to increase the frequency resolution of the wavenumber spectra. The spectra of zone (a) in the center column of Figure 4.13 shows that the highest amplitude wavenumber increases from 60 to about 75 when moving $5.08 \mathrm{~cm}$ (2 in) downstream. This effect is based on the previous observation that the measured vortex paths appear under varying inclination angles relative to the constant azimuth rays. The wavenumber of the roughness-excited cross-flow vortices most likely will not increase much more further downstream because the inclination of the vortex paths became less at higher $\mathrm{x}$-Reynolds numbers, and became nearly parallel to the constant azimuth rays of the cone.

The wave number spectra of zone (b) in the right hand column of Figure 4.13 show the dominant wavenumbers in the zone that were not affected by the $m=68$ dimples. The wavenumbers are about $m=16$ smaller than in the (a)-zones, and 

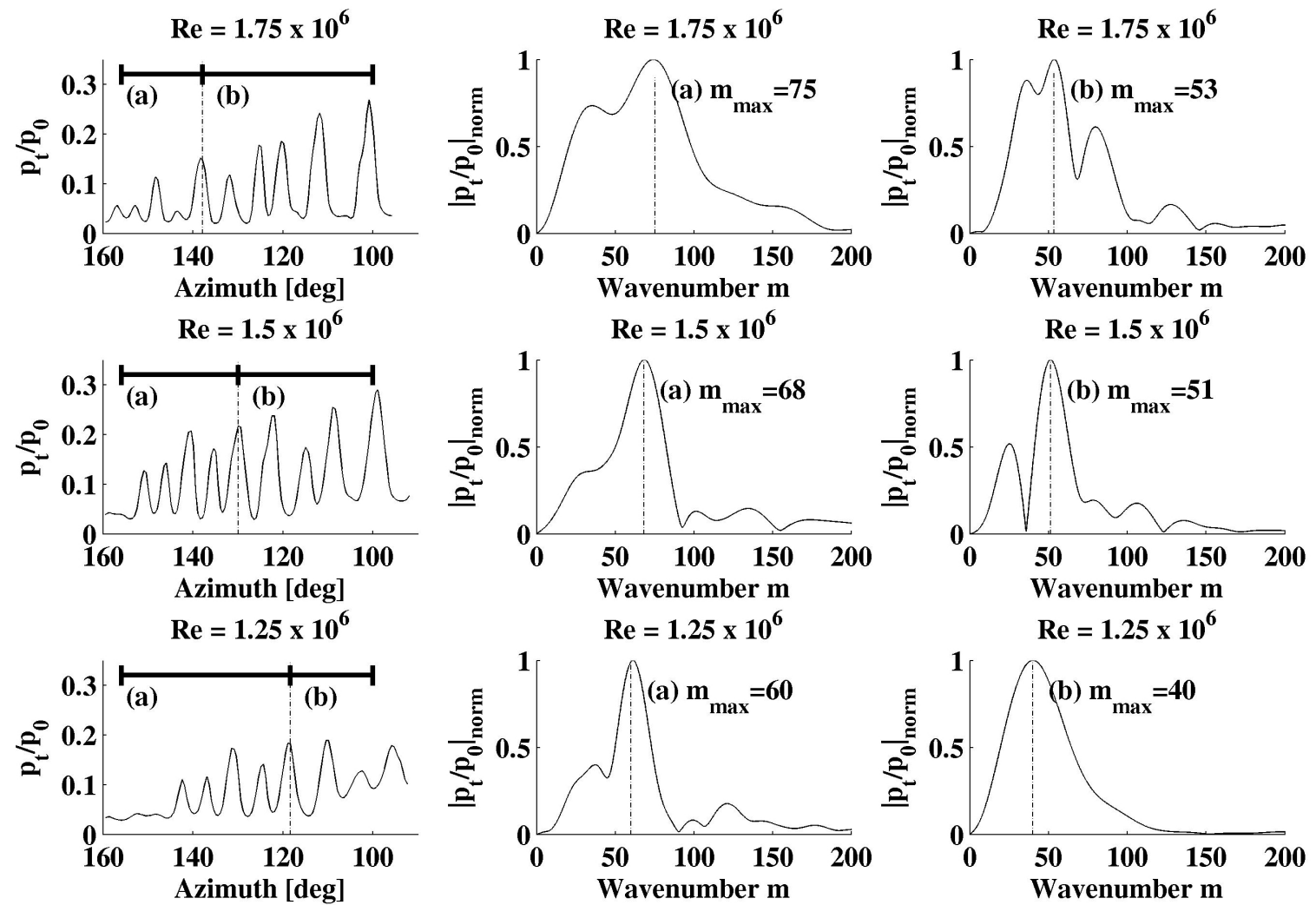

Figure 4.13. Comparison of the dominant wavenumbers with the $m=68$ dimple tip at $172.4 \mathrm{kPa}$ (25 psia) for different Reynolds numbers at $0.05 \mathrm{~mm}$ (0.002 in) height above surface. (a) and (b) correspond to the zones wind- and leeward of the parting vortex trace. 
show the same increasing trend with Reynolds number. This demonstrates that the parting vortex trajectory was correctly identified since there is a distinct difference in wavenumbers between zones (a) and (b).

The increase in wavenumber with increasing downstream axial location was initially suspected from the spectral analysis of the surface flow visualization images. These had indicated the generation of higher wave numbers with increasing $\mathrm{x}$-Reynolds numbers. This was confirmed in the Pitot probe traverses. The same effect was shown in Saric's low speed cross-flow measurements [45], where initially excited stationary cross-flow modes would generate superharmonics with higher wavenumbers. Lower wave numbers, subharmonics, were not observed by Saric or in the present results.

\subsubsection{Repeatability}

Successive measurements of the azimuthal pressure distributions at on e xlocation and stagnation pressure were performed to document the repeatability of the results. Figure 4.14 shows the result of multiple experiments with the baseline (smooth) cone tip, and with the $m=68$ tip.

The basic idea in the repeatability measurements is that the initial amplitudes generated by the patterned roughness is larger than that produced by random surface roughness. Thus, the stationary cross-flow vortices produced by the patterned roughness should be at fixed azimuthal locations on the cone. In contrast, the cross-flow vortices produced by the smooth tip will change in amplitude and location from run to run due to random roughness such as uncontrolled dust. The experiments confirmed this supposition.

The repeatability results with the smooth tip are shown in the left part of 

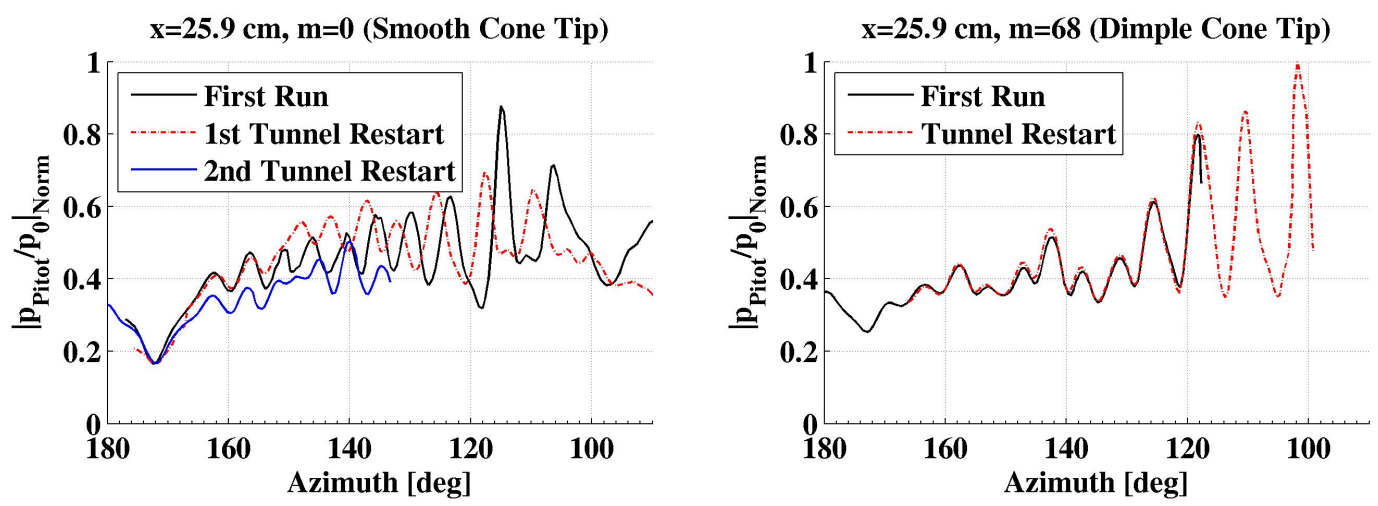

Figure 4.14. Repeated measurements of azimuthal pressure distributions at constant axial position. The left hand side shows the non-repeatability for the smooth tip and a well repeatable pattern for the $m=68$ cone tip. $\mathrm{P}_{0}=172.4 \mathrm{kPa}(25 \mathrm{psi})$

Figure 4.14. The peaks in the azimuthal pressure distribution, which were indicative of the stationary cross-flow vortices, were not aligned from run to run. In contrast, the peaks in the pressure distributions aligned perfectly with the $m=68$ roughness.

A wavenumber analysis of the repeated Pitot probe measurements is shown in Figure 4.15. The original pressure data series are high pass filtered and then normalized with the largest value of all $m=0$ or respectively $m=68$ pressure measurements. This data is shown in the left hand column of the figure. The right hand column represents the wave number spectra of the corresponding pressure data series. The spectral analysis was performed over the entire length of the data set, and over the length of the shortest data set in the group. For the smooth tip, the measurements that cover the entire range from $90^{\circ}<\theta<180^{\circ}$ indicate that dominant wavenumbers were around $m=50$. If the azimuthal range was limited to the length of the shortest data set, the dominant wave number varied from 
$m=50$ to 60 , depending on the data set.

With the $m=68$ cone tip, the dominant spectral peak was that of the patterned roughness. This signifies the repeatability in the wave number receptivity. The parting vortex line was most likely located at approximately $\theta=140^{\circ}$ based on $\mathrm{x}=25.4 \mathrm{~cm}(10 \mathrm{in})$ from the previous data in Figure 4.11 . The repeated azimuthal pressure measurements even show excellent agreement in the region that was on the windward side of the parting vortex line. This might be due to some azimuthal spreading of the cross-flow vortices in the downstream direction. This spreading broadens the effect of the upstream roughness. This was the ultimate test that the boundary layer was receptive to the patterned roughness.

Another test on the receptivity of the stationary cross-flow modes to the patterned roughness is shown in Figure 4.16. This documents traverses of the Pitot probe in the axial direction for a constant azimuthal angle on the cone for the $m=68$ tip. In this case, the unit Reynolds number was increased by increasing the stagnation pressure. The different unit Reynolds numbers will cause different growth rates of the excited cross-flow vortices. Therefore the amplitude of the pressure peaks is not expected to be the same between cases. However, the axial locations of the peaks is expected to be the same. Figure 4.16 shows that this was the case. This further signifies that the boundary layer was receptive to the patterned roughness for the full range of Reynolds numbers that were examined.

\subsection{Transition Front Detection}

Because the axial range of motion for the Pitot probe was limited from about $7.62 \mathrm{~cm}$ (3 in) to about $25.4 \mathrm{~cm}$ (10 in), the turbulent transition location needed to 

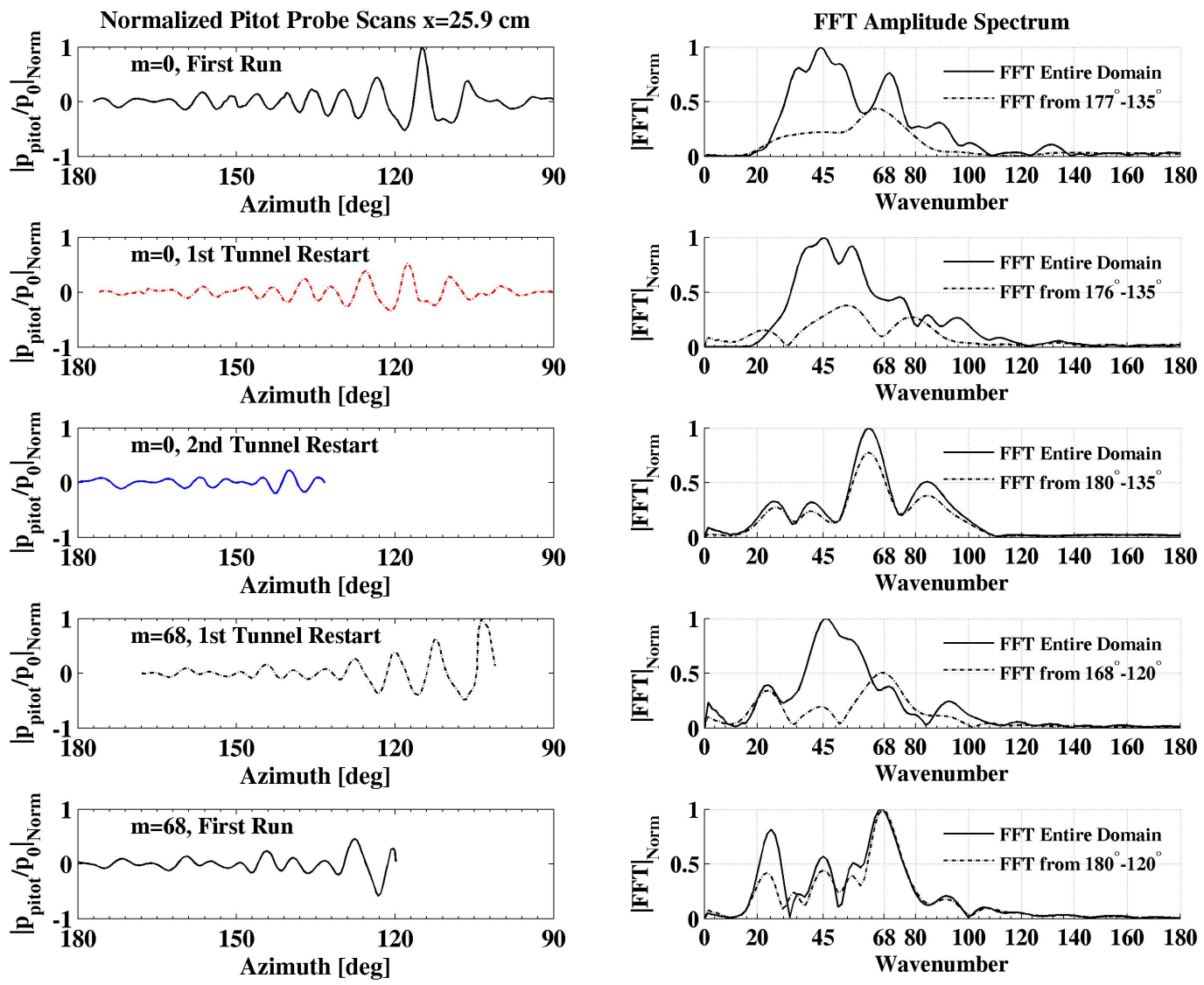

Figure 4.15. Wavenumber analysis of the repeated Pitot probe measurements with the $m=0$ and $m=68$ cone tip at $\mathrm{x}=25.4 \mathrm{~cm}$ (10 in).

The total pressure is $\mathrm{P}_{0}=172.4 \mathrm{kPa}(25 \mathrm{psi})$ 


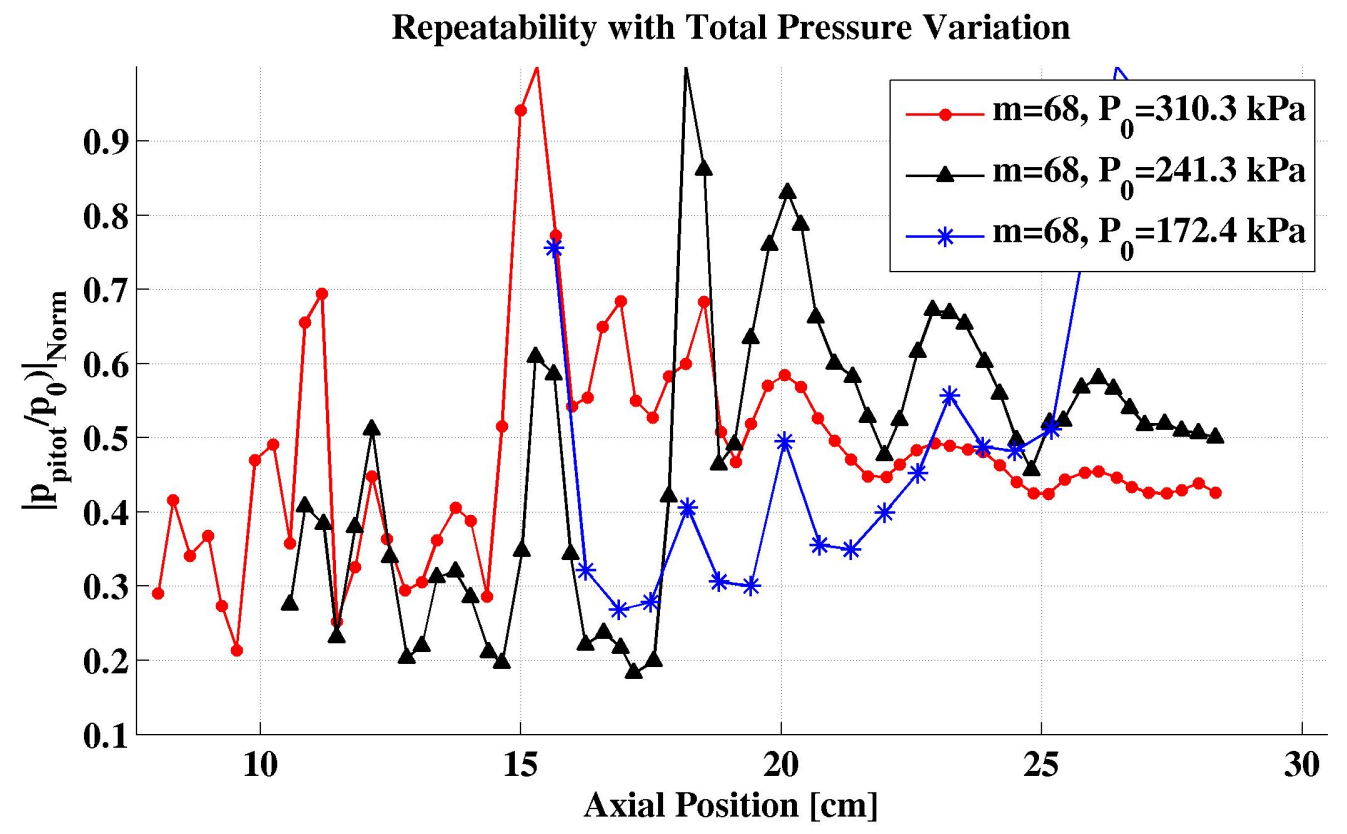

Figure 4.16. Pitot probe measurements at constant azimuthal angle $\left(\theta=120^{\circ}\right)$ and three different free stream unit Reynolds numbers:

$R e_{25 p s i}=0.25 \times 10^{6} \mathrm{in}^{-1}, R e_{35 p s i}=0.35 \times 10^{6} \mathrm{in}^{-1}$ and $R e_{45 p s i}=0.45 \times 10^{6} \mathrm{in}^{-1}$. 
occur within this axial range to be documented. Based on the flow visualization images from Figure 4.1, a tunnel stagnation pressure of $310.3 \mathrm{kPa}$ (45 psi) was chosen because turbulent transition appeared to occur between $27.9 \mathrm{~cm}$ (11 in) to $35.6 \mathrm{~cm}$ (14 in) downstream of the cone tip at that stagnation pressure.

Pitot pressure measurements were taken on the surface of the cone in order to map the development of a few stationary cross-flow vortices. The azimuthal Pitot probe measurements for the transition front detection were done by placing the probe on the surface of the cone. This was different from the receptivity measurements, where the Pitot probe was kept at a constant height above the surface. By placing the probe as close as possible to the surface, the measured pressure could be related to the wall shear stress in a manner that is similar to a Preston probe. Details about Preston probe measurements are given later in this section.

Figure 4.17 shows the Pitot probe data for seven different axial positions of $1.7 \times 10^{6}<R e_{x}<3.5 \times 10^{6}$, and azimuthal angles covering approximately $20^{\circ}$. The top part of the figure shows the Pitot probe data for the $m=45$ case. The bottom part of the figure shows the data for the $m=68$ case. The space between each measured axial position is interpolated to form a continuous sheet of the pressure distribution. Figure 4.18 shows the axial interpolated pressure traces. The height and coloring of the 3-D pressure sheets represents the total pressure, normalized with the stagnation pressure in the wind tunnel. The interpolation involved three steps. Each pressure maximum of the azimuthal scans at each axial position was assigned to a certain cross-flow vortex. Next a linear interpolation was used to transform the location of the maxima of each azimuthal scan to the same location as the starting angles of their respective reference cross-flow vortex. If the maxima of each cross-flow vortex were aligned, a 2-D interpolation in the 


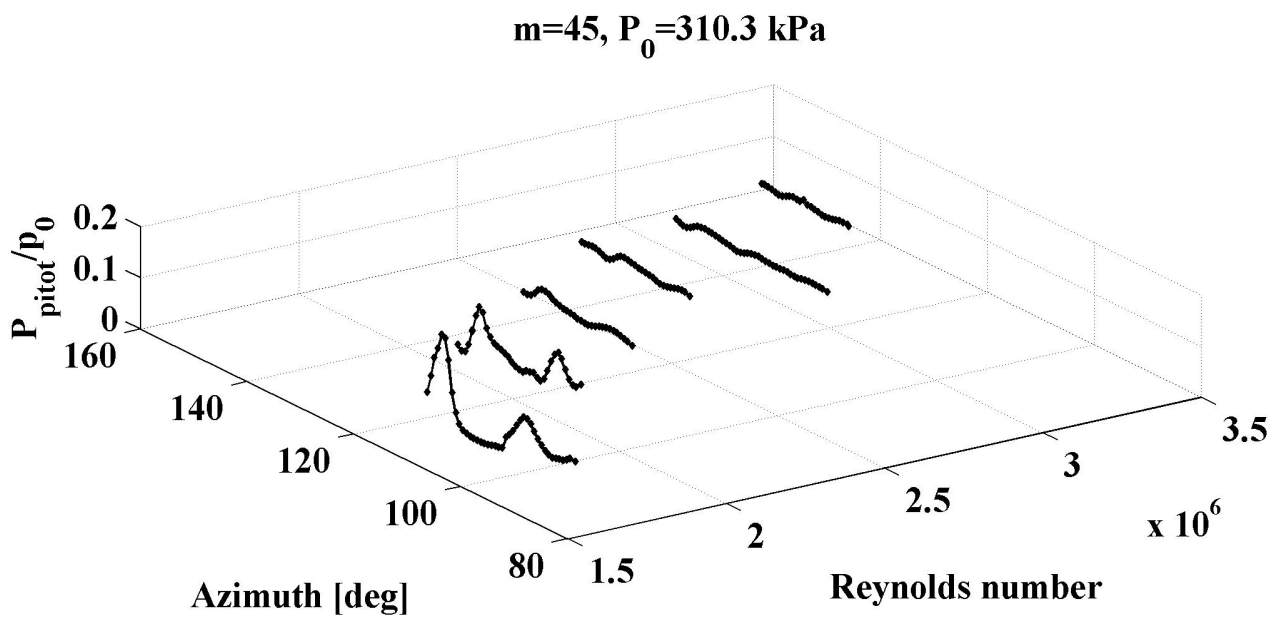

$$
\mathrm{m}=68, \mathrm{P}_{0}=310.3 \mathrm{kPa}
$$

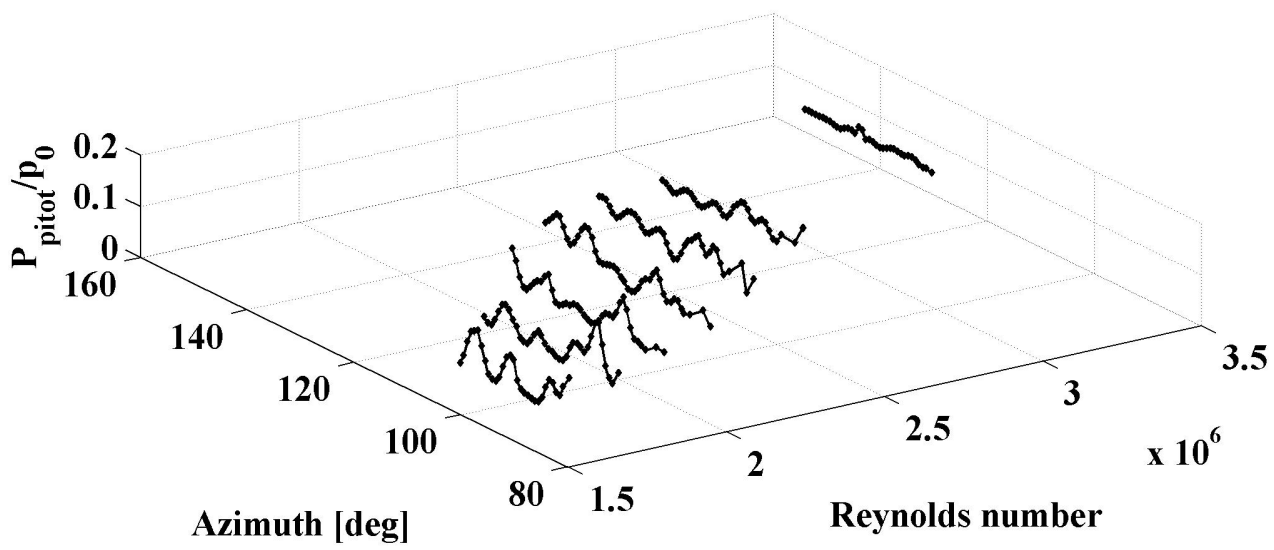

Figure 4.17. Pitot probe measurements for $m=45$ (top) and $m=68$ (bottom) patterned roughness. $\mathrm{P}_{0}=310.3 \mathrm{kPa}$ (45 psi) 


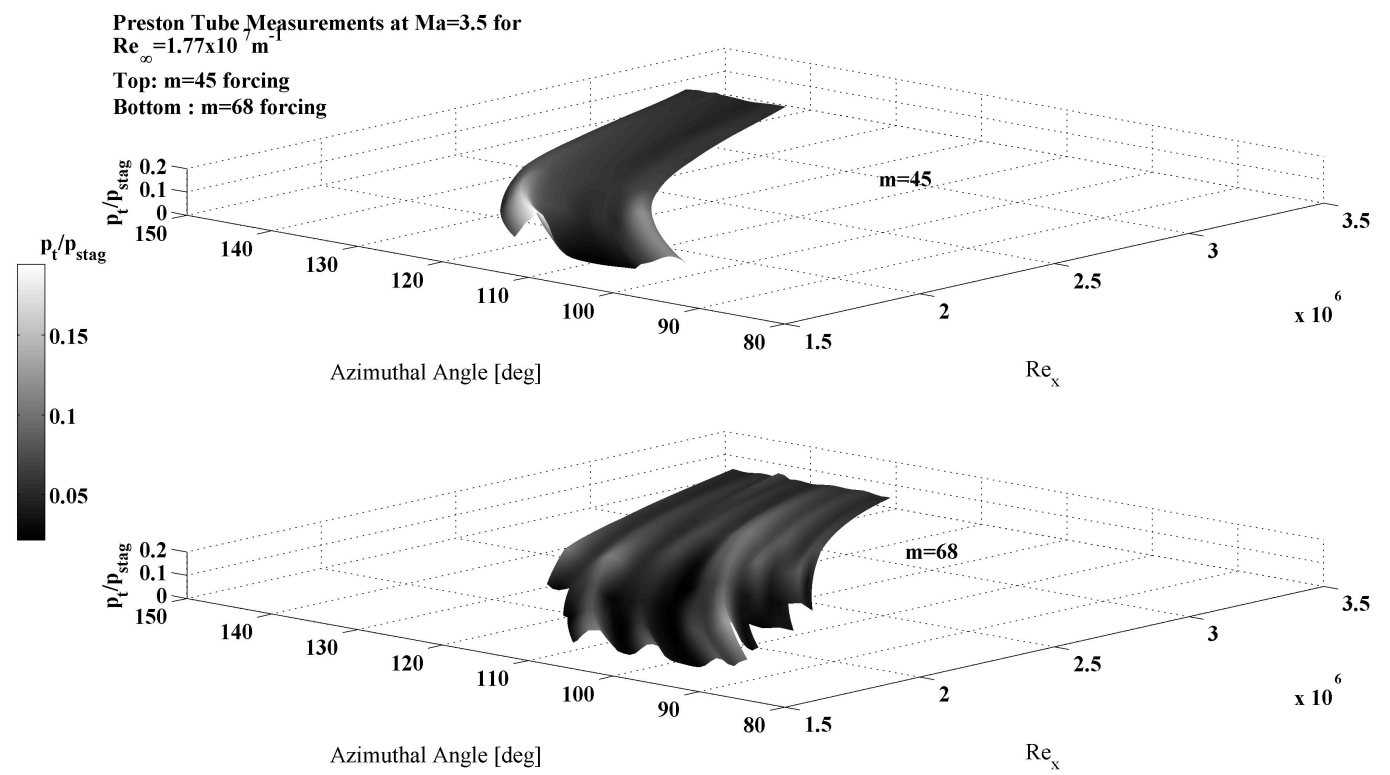

Figure 4.18. Preston probe measurements for $m=45$ (top) and $m=68$ (bottom) forcing at $\mathrm{Ma}=3.5$ and a freestream unit Reynolds number of $1.77 \times 10^{7} \mathrm{~m}^{-1}\left(4.5 \times 10^{5} \mathrm{in}^{-1}\right)$ and $\mathrm{P}_{0}=310.3 \mathrm{kPa}(45 \mathrm{psi})$. The heights correspond to the normalized total pressure at the probe tip.

axial direction was performed. Finally the whole 2-D field was back-transformed so that the original spacing between the maxima was restored. The points from the 2-D interpolation in between each axial location were back-transformed according to a third order polynomial fit to the interpolated cross-flow vortex traces in the axial direction.

There are three major pieces of information that can be extracted from Figure 4.18. These are the approximate wavenumbers of the mapped vortical structures, the amplitudes of the measured stagnation pressures of the cross-flow vortices, and the paths of the cross-flow vortices.

A comparison of the axial development of the amplitudes of the two cases makes it obvious that the $m=45$ case decays faster than the $m=68$ case. In or- 


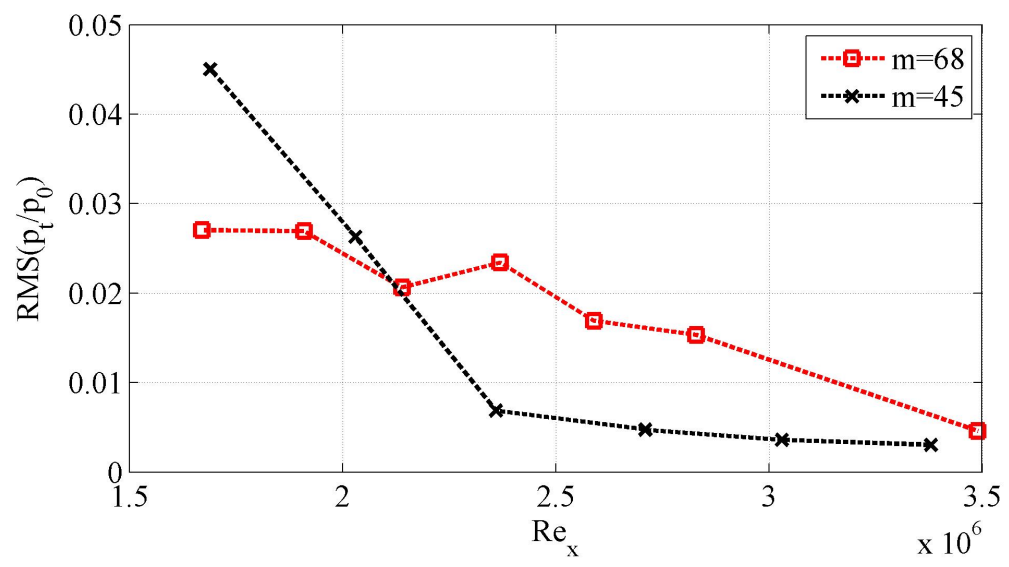

Figure 4.19. Axial development of the RMS of the pressure fluctuations in the azimuthal direction that is associated with the cross-flow vortices.

der to quantify the decay rate, the RMS of the pressure variation in the azimuthal direction that is associated with the mean flow distortion of cross-flow vortices, was calculated at each of the axial locations. The result is shown in Figure 4.19. This shows that the coherent mean flow distortion associated with the cross-flow vortices decays much more quickly in the axial direction with the $m=45$ case. As such, it is an indication that the boundary layer is transitioning to turbulence faster compared to the $m=68$ case. Similar conclusions were drawn from Naphthalene flow visualizations as done by Saric [44] (Figure 1.3). He concluded that if the streaks that represent cross-flow vortices disappear, the boundary layer became turbulent [44, 47]. Based on this assumption, turbulent transition is delayed by the $m=68$ subcritical roughness compared to the controlled baseline critical roughness, $m=45$. Based on this comparison, transition to turbulence was delayed by almost $R e_{x}=1 \times 10^{6}$.

Another method to detect turbulent transition is based on Preston tube measurements as done in Fisher's supersonic free flight experiments [18] or King's 


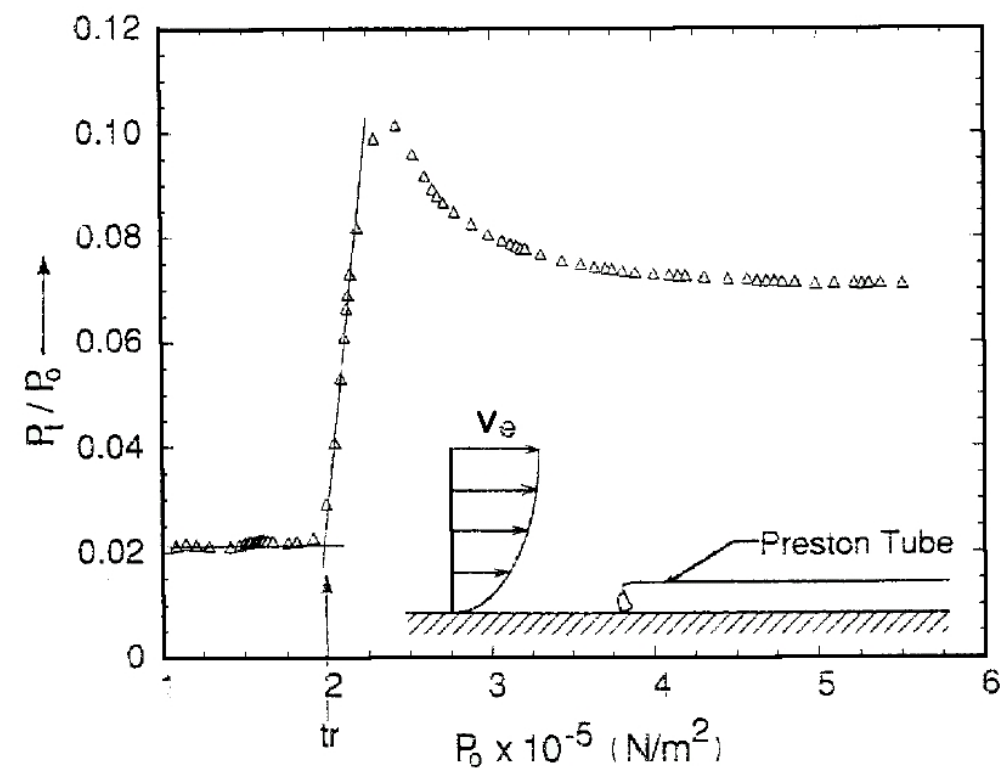

Figure 4.20. Preston probe method showing sharp rise in measured total pressure with increasing free stream Reynolds number at the location of transition onset. Figure was taken from [28].

NASA Mach 3.5 SLDT measurements. King [28] used a Preston probe that was mounted on the surface of a $5^{\circ}$ half-angle cone at a fixed location, and varied the free stream unit Reynolds number of a Mach 3.5 flow. When the turbulent transition front moved across the Preston tube, it registered a sharp rise in the total pressure. An example of this effect is shown in Figure 4.20. Similar data can be extracted from the surface Pitot probe measurements for the $m=45$ and $m=68$ roughness cases. The main challenge is that there is a coherent mean flow distortion that is spatially fixed due to the patterned roughness. It is therefore more difficult to distinguish an increase of the total pressure between the thickening of the boundary layer from the cross-flow vortices and the transition from laminar to turbulent flow.

In order to overcome this issue, the change in the total pressure was performed 


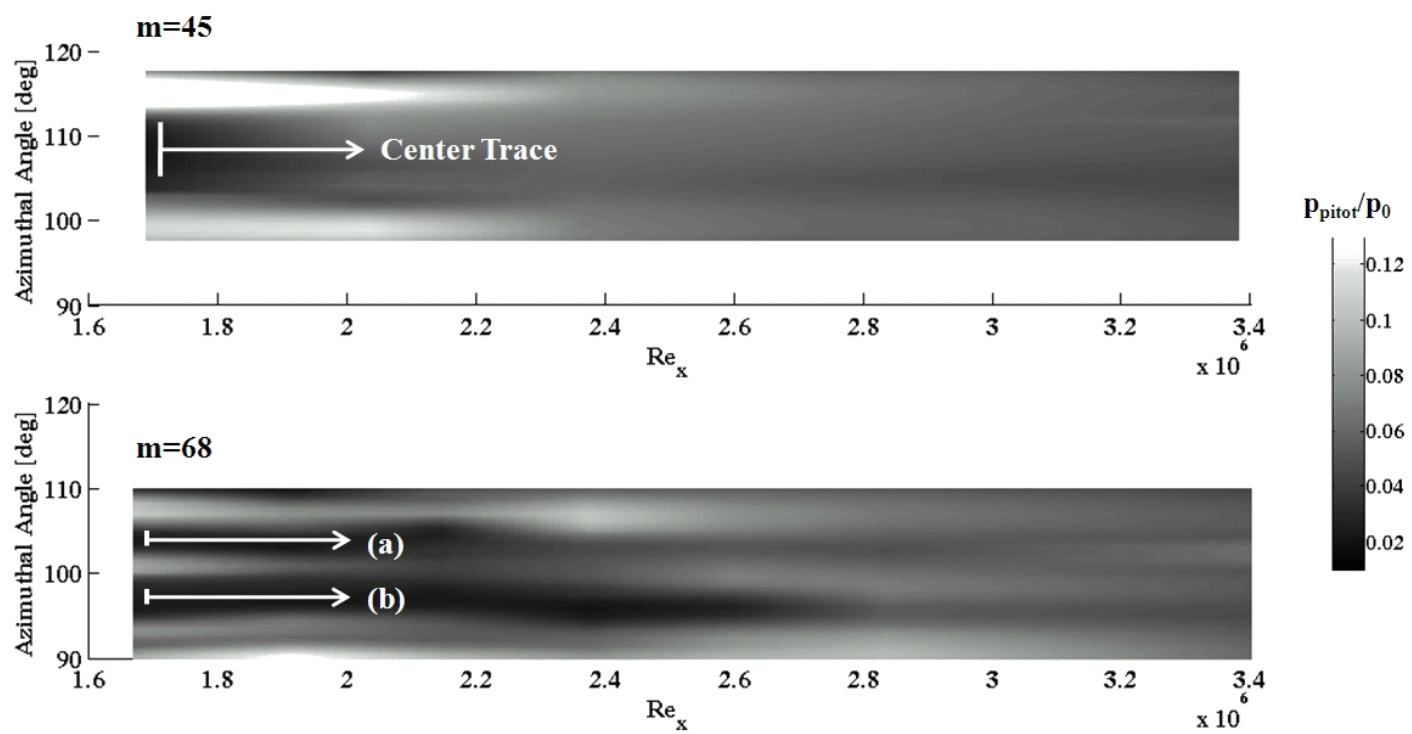

Figure 4.21. Straightened Preston probe scans showing the normalized total pressures and paths of the analyzed pressure traces for the detection of laminar-turbulent transition. $\mathrm{P}_{0}=310.3 \mathrm{kPa}$ (45 psi)

by moving in an axial direction that was parallel to the path of the cross-flow vortices. To aid in doing that, the curvature of the cross-flow vortices was removed. The result is shown in Figure 4.21 .

The change in pressure in the axial direction close to the surface of the cone was then taken in a pressure minimum. The arrows in Figure 4.21 indicate the chosen pressure minima and are labeled with (a) and (b). The axial development of the pressure minima is shown for the $m=45$ case in Figure 4.22 and for the $m=68$ case in Figure 4.23 . For the $m=45$ case, the normalized pressure starts at approximately 0.022 and rises quickly to a peak value that is approximately three times larger than the starting value. After that, the normalized pressure falls off slowly. This is similar to that which was shown in Figure 4.20. A first order polynomial was fit to the fast rising part of the pressure distribution. The 
location where the fitted curve intersected with the laminar transition pressure value was defined as the location of turbulent transition. The laminar pressure value for the $m=68$ case was estimated by averaging all pressure values before the rising pressure. The black dash-dotted lines in Figures 4.22 and 4.23 indicate the interval over which $95 \%$ of the laminar value lies within. The laminar pressure for the $m=45$ case was chosen to be the lowest pressure measured. This was done because the measurement did not extend far enough upstream to capture where the pressure leveled. It was then assumed that the uncertainty in the estimated pressure was the same as for the $m=68$ case.

Figure 4.22 also shows the variation in the intercept of the fitted portion of the rising pressure with the laminar pressure. It ranges from $1.61 \times 10^{6}$ (a) to about $1.71 \times 10^{6}$ (c) with a mean value of $1.67 \times 10^{6}$ (b) for the $m=45$ case.

The azimuthal spacing between each axial pressure trace was $0.5^{\circ}$. The azimuthal spacing between the cross-flow vortices was smaller for the $m=68$ case so that there were less axial pressure distributions available to determine the transition location. The result is shown in Figure 4.23. Again the rise in the pressure was fit and extrapolated to find the intersection with the laminar pressure. The two fitted sets (a) and (b) correspond to the two visible low pressure zones as indicated in Figure 4.21. The transition Reynolds numbers are $2.1 \times 10^{6}$ for trace (a) and $2.5 \times 10^{6}$ for trace (b).

In order to be able to directly compare the transition locations for the $m=45$ and $m=68$ cases, the turbulent transition locations determined from Figures 4.23 and 4.22 were plotted along with the stationary cross-flow vortex trajectories in Figure 4.24. It can be seen that over the range of measured azimuthal angles, the transition Reynolds number increased by $0.4 \times 10^{6}$ to $0.8 \times 10^{6}$ with the $m=68$ 


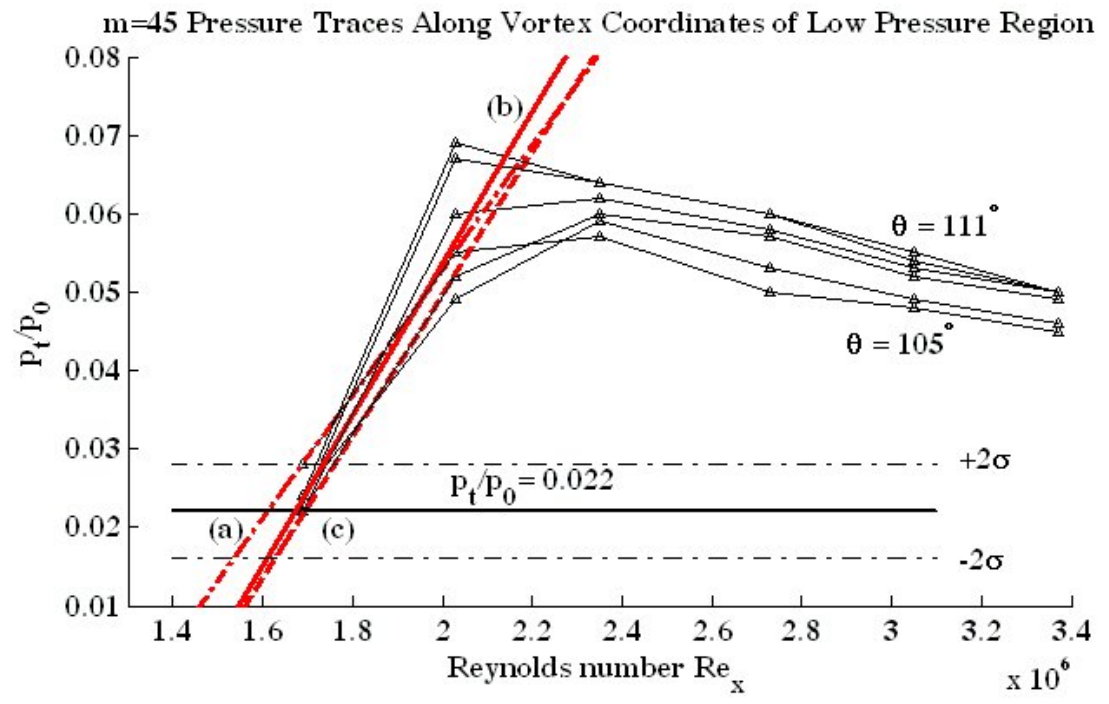

Figure 4.22. $m=45$ pressure traces parallel to the cross-flow vortex structure centered between the two visible pressure maxima. $0.5^{\circ}$ azimuthal spacing between each trace, covering approximately $6^{\circ}$ from $105^{\circ}$ to $111^{\circ}$ in azimuthal direction. The dashed red line is a linear curve fitted to the slope of the pressure signal. The intersection with $p_{t} / p_{0}=0.022$ is defined as laminar turbulent transition onset. 


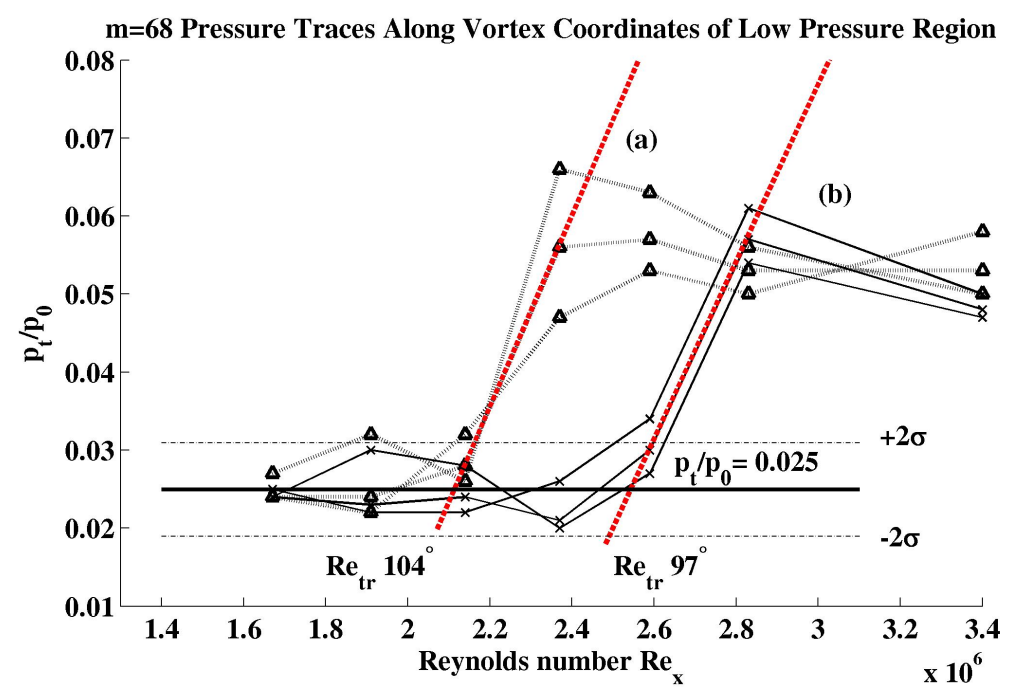

Figure 4.23. $m=68$ normalized pressure traces parallel to the cross-flow vortex structure. There are two low pressure regions (a) and (b) for which the transition Reynolds number $R e_{t r}$ is estimated. The angles correspond to the initial angle where the measurement of the traces was started.

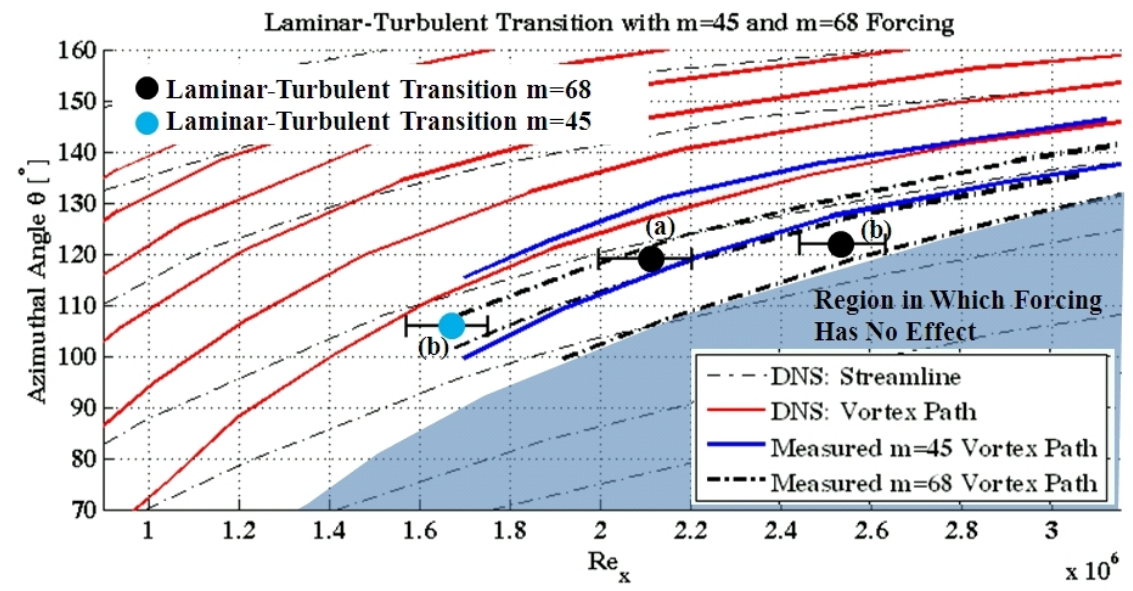

Figure 4.24. Shown are the laminar-turbulent transition locations for the $m=45$ and $m=68$ distributed roughness tips including error bars. The black circular dots (a) and (b) correspond to the traces in Figure 4.21 with $m=68$ forcing. The blue dot corresponds to the location identified in Figure 4.22 with $m=45$ forcing. 
subcritical forcing. The turbulent transition location moves upstream at larger azimuthal angles. The amount of data is not large enough to compile a complete transition front, however the results indicate that the subcritical mode forcing moved the turbulent transition location downstream by $25 \%$ to $50 \%$.

Figure 4.24 includes error bars that are based on the uncertainty in the estimated laminar pressure measurement, as well as on the range of possible extrapolated transition locations. The increase in the transition Reynolds number due to the subcritical mode forcing is well within the error bars.

A more complete picture of the expected laminar turbulent transition front is shown in King's 1991 paper [28]. As described earlier, transition to turbulence occurs further upstream for the leeward side of the cone compared to the windward side. A comparison with Balakumar's N-factor calculations [3] gives a similar picture for the windward side transition front, but not for the leeward side. $\mathrm{N}$-factors of the stationary and travelling cross-flow modes become smaller when moving towards the leeward ray at $180^{\circ}$, suggesting that transition to turbulence starts first around azimuthal angles of $130^{\circ}$. The discrepancy between the actually measured transition fronts by King, and Balakumar's N-factor calculations close to the leeward side at $180^{\circ}$, is possibly due to the effect of uncontrolled surface roughness on the cone.

In order to project the laminar-turbulent transition front of the present experiments to higher azimuthal angles, the N-factor at the measured turbulent transition location was estimated from Balakumar's N-factor calculation, and defined as the transition N-factor. The projected laminar-turbulent transition front for the $m=45$ and $m=68$ cases based on Balakumar's N-factor calculations is then shown in Figure 4.25. This suggests an N-factor of 6 for the $m=45$ critical 

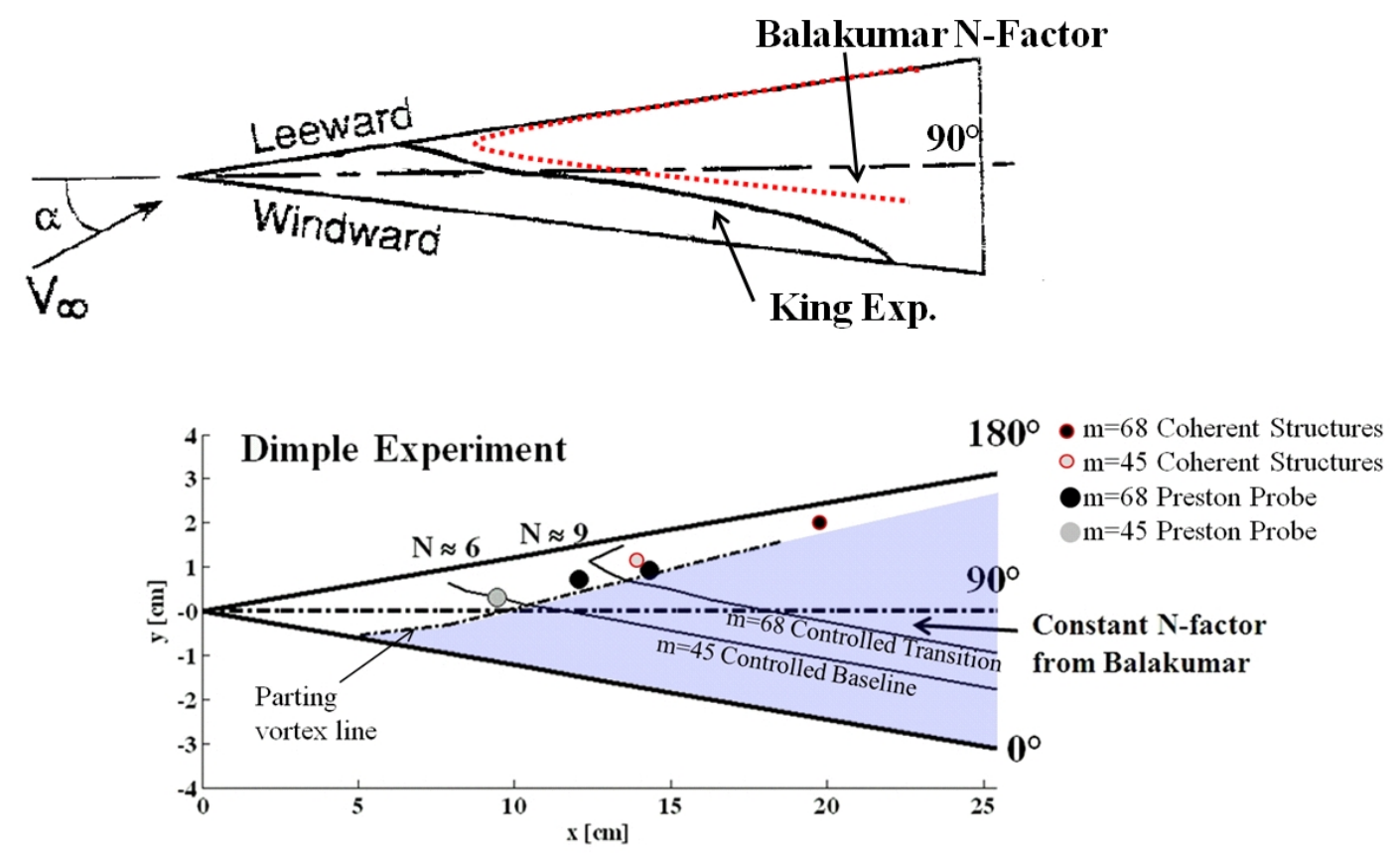

Figure 4.25. The top figure is adapted from King [28] and shows the qualitative shape of the transition front. Added is the shape of the transition front based on Balakumar's linear stability analysis [3]. The bottom figure shows the Preston probe and coherent structure based turbulent transition locations including curves of constant turbulent transition N-factors.

wavenumber case, and a higher N-factor of 9 for the $m=68$ subcritical wave number case.

The last set of information that can be extracted from the azimuthal Preston probe data is its wavenumber content. This is shown in Figure 4.26 for the $m=45$ dimple cone tip and in Figure 4.27 with the $m=68$ dimple cone tip. Each figure contains the development of the wavenumber spectra for multiple axial locations, following the evolution of the measured subset of stationary cross-flow vortices downstream. The amplitudes in the wavenumber spectra are normalized by the largest amplitude occurring for the $m=68$ and $m=45$ case respectively. 


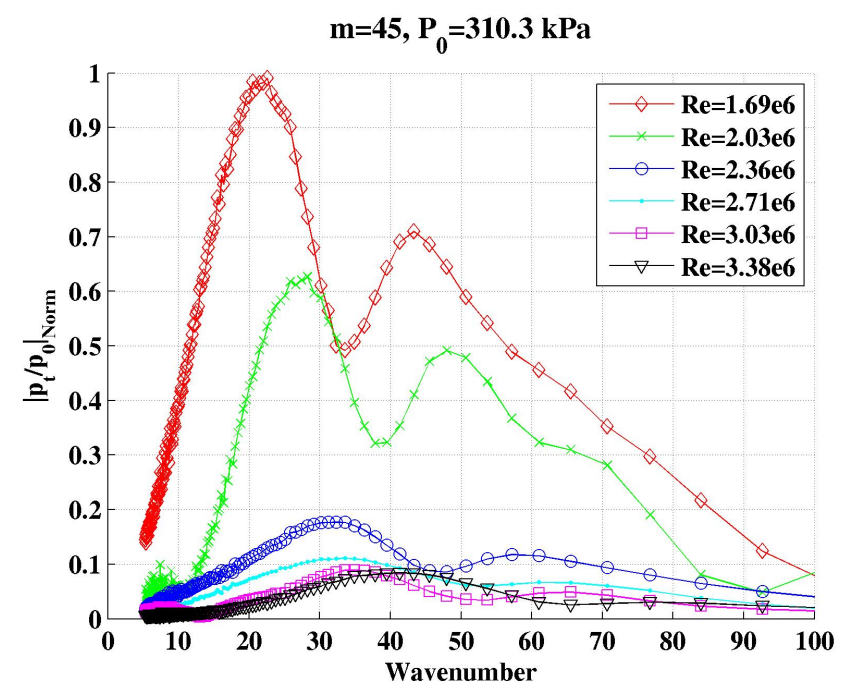

Figure 4.26. Wavenumber amplitude spectrum of the normalized total pressures from Figure 4.17 with the $m=45$ cone tip

The largest amplitudes occur in both cases at the most upstream or smallest Reynolds number location. As demonstrated before, the amplitudes decay then at larger Reynolds numbers. The dominant wavenumbers in case of the $m=45$ dimple cone tip as seen in Figure 4.26 are centered around 20 and 45 for Reynolds numbers of $1.69 \times 10^{6}$ and $2.03 \times 10^{6}$. At a Reynolds number of 2.36 million, the amplitudes decayed to less than $20 \%$ of their initial values at $\operatorname{Re}=1.69 \times 10^{6}$ and show a relatively broad spectral content, centered around a wave number of 40 . Based on the previously identified turbulent transition location measurements, the entire range of analyzed Reynolds numbers corresponds to the region of turbulent transition.

The dominant wavenumbers with the $m=68$ dimple cone tip as seen in Figure 4.27 are centered around a wavenumber of $m=55$ in the range of $1.67 \times 10^{6}<$ $R e<2.37 \times 10^{6}$. The dominant wavenumber then appears at a higher value 


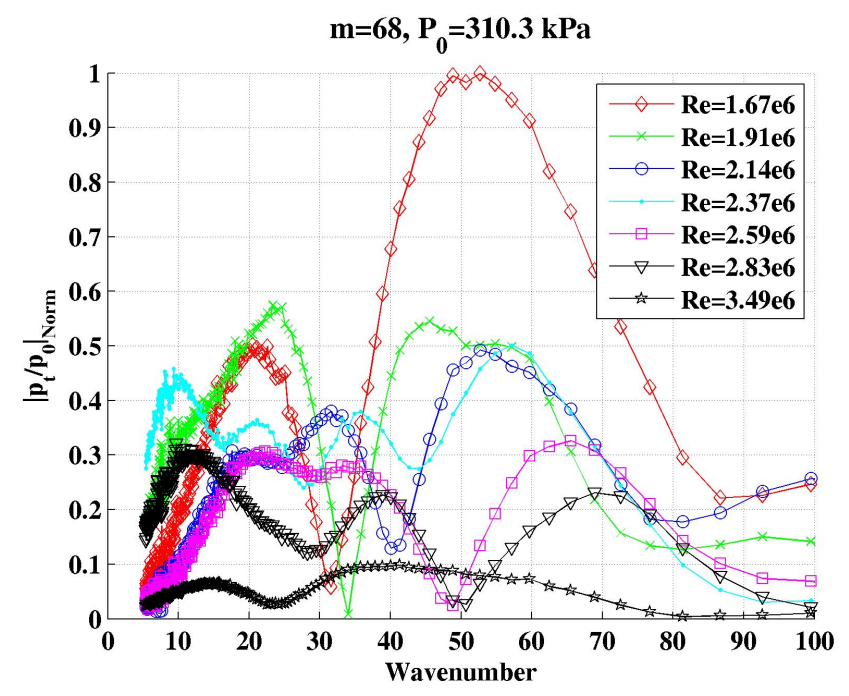

Figure 4.27. Wavenumber amplitude spectrum of the normalized total pressures from Figure 4.17 with the $m=68$ cone tip

centered around $m=70$ for $2.59 \times 10^{6}<R e<2.83 \times 10^{6}$. Finally at the most downstream or largest Reynolds number location, the spectrum is centered at a wavenumber of $m=40$. Additionally some broad band low wavenumber content between $m=10$ and $m=30$ is present.

As observed in flow visualizations by Saric [44, stationary cross-flow modes with wavenumbers lower than the initially dominant wavenumber can be present before the onset of turbulence. This is what happened in case of the $m=45 \mathrm{dim}$ ple case. The largest amplitude wave number is centered around 20, whereas the excited wavelength of $m=45$ has a $30 \%$ smaller amplitude. Since turbulent transition was present in the entire range of Reynolds numbers that was covered by this set of measurements, this corresponds to the evolution of the low wavenumber mode that was observed by Saric before the turbulent boundary layer appeared. Finally, even this mode disappears and the wavenumber spectrum becomes broad. 
A very similar behavior can be observed with the $m=68$ excitation. As a reminder, the onset of transition was estimated to be at a Reynolds number between 2.2 and $2.5 \times 10^{6}$. At the farthest upstream location, the amplitude of the excited mode was about 2-times larger than the amplitudes at the lower wavenumbers, and the $m=45$ mode was completely suppressed. This ratio drops to about 1.25 when approaching the location of turbulent transition at $R e=2.37 \times 10^{6}$. At $R e=2.59 \times 10^{6}$, the band from $m=10$ to $m=30$ and the excited wavenumber at $m=68$ are all of equal amplitude. This is the furthest location where turbulent transition onset was identified. After that, the low wavenumber amplitudes surpass the excited wavenumber amplitude and the spectrum becomes broad at the farthest downstream location. Therefore, the evolution of the wavenumber spectrum during turbulent transition was similar to that observed in Saric's subsonic stationary cross-flow mode dominated transition experiment. 


\section{CHAPTER 5}

\section{RESULTS WITH DBD PLASMA ROUGHNESS}

The two main objectives of the experiments with passive distributed roughness were to show receptivity of the stationary cross-flow instability to the patterned roughness, and that subcritical wavenumber forcing delayed the onset of turbulent transition. In this chapter, the passive distributed roughness was replaced by a DBD plasma actuator array. Again both critical wavenumber excitation at $m=45$, and subcritical wavenumber forcing at $m=68$ were investigated.

\subsection{Pitot Tube Surveys: Receptivity to Excitation Wavenumber}

The most important part of the experiment was to show that the cross-flow instability was receptive to the DBD plasma actuator array. For this purpose, azimuthal scans at various axial locations were performed with a Pitot probe placed $0.04 \mathrm{~mm}$ above the cone surface. Based on Balakumar's DNS simulations of the boundary layer [3], the Pitot probe height was within the boundary layer thickness, which was estimated to be $0.5 \mathrm{~mm}$ (0.02 in) to $0.76 \mathrm{~mm}$ (0.03 in). The azimuthal pressure scans were performed at a tunnel total pressure of $172.4 \mathrm{kPa}$ (25 psia). Five different axial locations from $\mathrm{x}=12.7 \mathrm{~cm}$ ( 5 in) to $\mathrm{x}=22.86 \mathrm{~cm}$ (9 in) in $2.54 \mathrm{~cm}$ ( 1 in) increments were examined. Plasma-on and plasma-off cases were compared. The results are presented for the $m=45$ cone tip in Figure 5.1, 


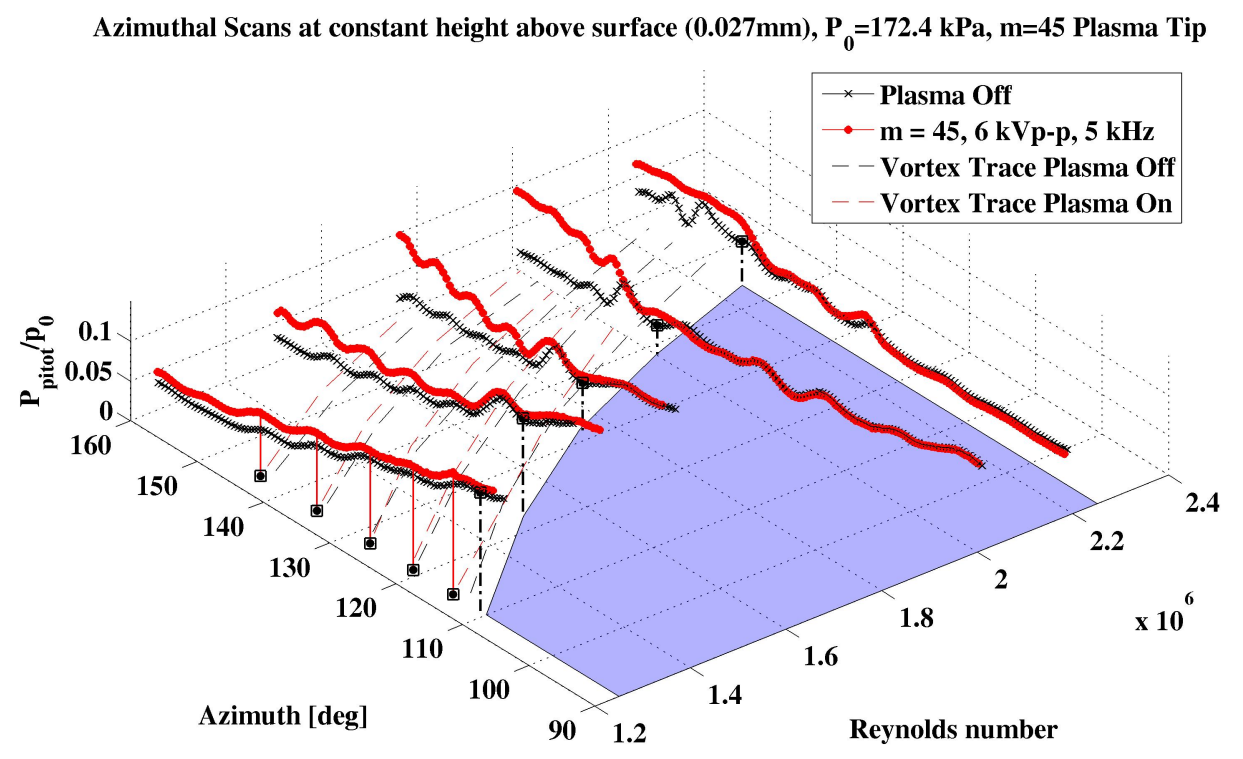

Figure 5.1. Pitot probe traces at $0.027 \mathrm{~mm}(0.0011 \mathrm{in})$ height above the surface, $\mathrm{P}_{0}=172.4 \mathrm{kPa}(25 \mathrm{psia}), T_{0}=311^{\circ} \mathrm{K}\left(100^{\circ} \mathrm{F}\right) \cdot m=45 \mathrm{DBD}$ plasma cone tip. Vortex traces are outlined with dashed lines. The blue area corresponds to the zone where forcing at $1.27 \mathrm{~cm}(0.5 \mathrm{in})$ has no influence (from Figure 4.11.) 
and for the $m=68$ cone tip in Figure 5.2. These show the total pressure in the boundary layer as a function of the azimuthal and axial locations. For each plasma roughness wave number, both plasma-off and plasma-on results are shown. The trajectories of the cross-flow vortices are indicated by drawing a curve through the pressure maxima. Finally the region where the effects of the roughness at $\mathrm{x}=1.27 \mathrm{~cm}(0.5 \mathrm{in})$ were outside of the parting vortex line are indicated by the blue shading.

As mentioned in the previous chapters, the azimuthal fluctuations in the total pressure in the boundary layer is indicative of the mean flow distortion caused by the stationary cross-flow mode. For the $m=45$ case in Figure 5.1, the amplitude with the plasma-on was always larger than with the plasma-off. The two amplitudes converge almost exactly along the parting vortex path. This is further evidence of its azimuthal location.

To address the question of whether the boundary layer was receptive to the DBD plasma actuator array, the azimuthal wave number of the pressure fluctuation for each case, were compared. This is first shown for three different streamwise locations for the $m=45$ case in Figures 5.3 to 5.5 . It is shown for the $m=68$ case in Figures 5.6 to 5.8. These figures show the azimuthal pressure distribution for the baseline (plasma off) and the plasma on conditions. The blue shaded region between $90^{\circ}<\theta<125^{\circ}$ is the azimuthal angles beyond the parting vortex line in which the mean flow path does not intersect the plasma actuator array at the tip. This is the same region that was indicated in Figures 5.1 and 5.2 . The bottom part of each figure are spectra of the pressure distribution. The spectral amplitudes have been normalized by the largest value of the plasma off spectra. This preserves the relative amplitude between the two spectra. The horizontal axis 


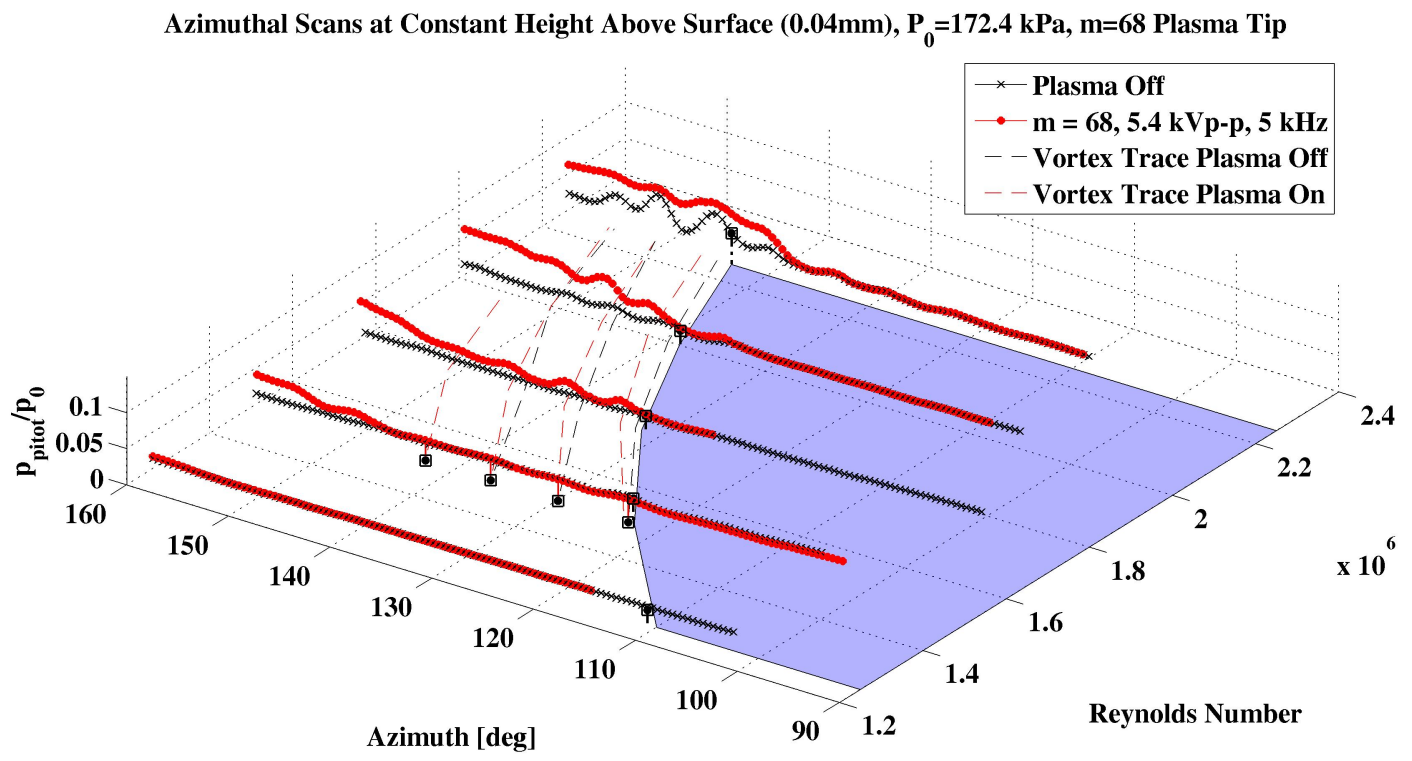

Figure 5.2. Pitot probe traces at $0.04 \mathrm{~mm}$ (0.0016 in) height above the surface, $\mathrm{P}_{0}=172.4 \mathrm{kPa}(25 \mathrm{psia}), T_{0}=100^{\circ} \mathrm{F} . m=68$ DBD plasma cone tip. Vortex traces are outlined with dashed lines. The blue area corresponds to the zone where forcing at $1.27 \mathrm{~cm}(0.5 \mathrm{in})$ has no influence (from figure 4.11.) 

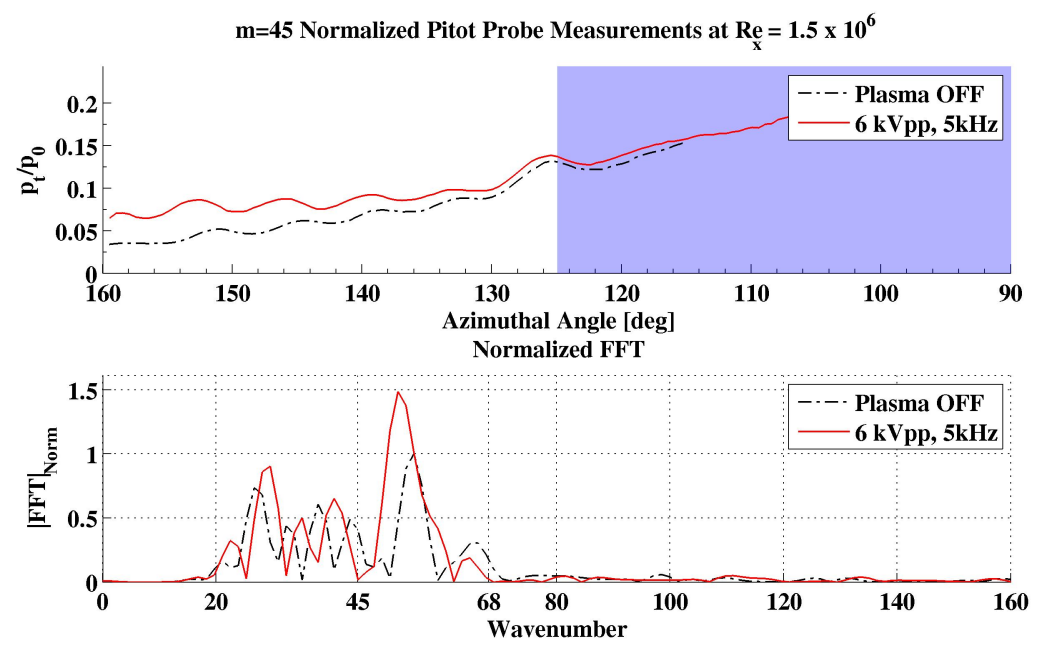

Figure 5.3. Azimuthal Pitot probe scans with the $m=45$ plasma cone tip at $\mathrm{x}=15.2 \mathrm{~cm}(6 \mathrm{in})$ and $R e_{x}=1.5 \times 10^{6}$. The FFT is applied to the white area of the Pitot probe data series. $\mathrm{P}_{0}=172.4 \mathrm{kPa}(25 \mathrm{psi})$

shows the wave number. This is a linear axis although tic marks are shown at $m=45$ and $m=68$. Details about how the spectra were processed are given in Appendix B.

Focusing first on the $m=45$ case, at the most upstream location shown in Figure 5.3. the plasma-on condition increased the amplitude in the most amplified range of azimuthal wave numbers, between 45 and 60, compared to the baseline. However there was no clear spectral peak at $m=45$ at this streamwise location. However, a spectral peak close to $m=45$ emerges at the next streamwise location as shown in Figure 5.4. In addition, the larger peak in the spectrum at the previous upstream location has shifted towards higher wave numbers. Finally at the furthest downstream location, the spectra in Figure 5.5 reveals a strong peak at $m=45$. Comparing the two spectra for plasma-off and plasma-on at this streamwise location, shows them to be similar, except with an additional peak at $m=45$ 

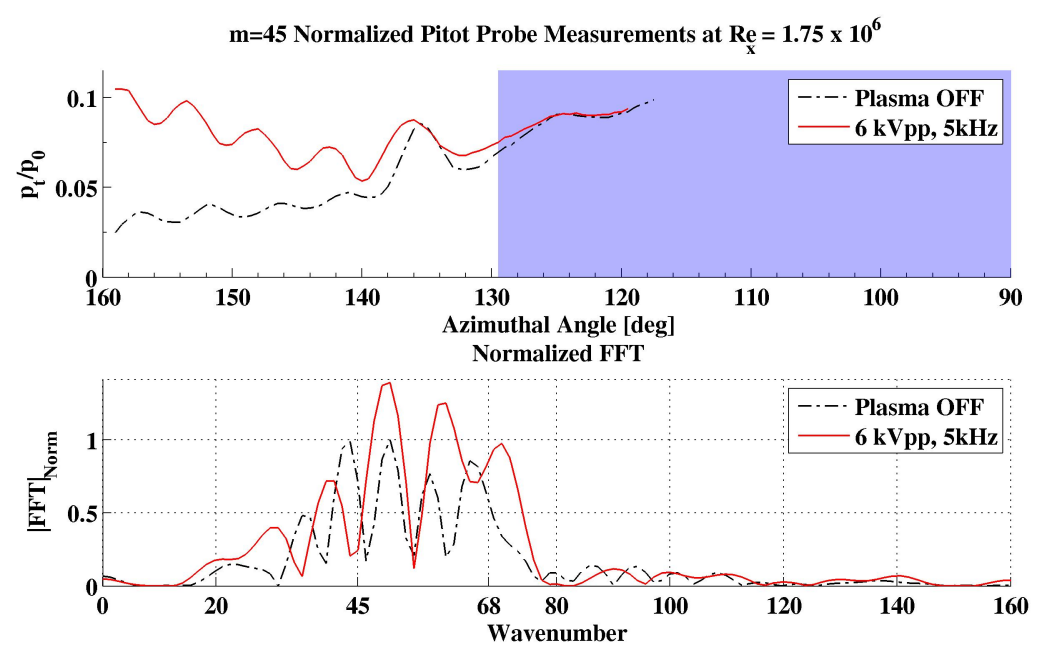

Figure 5.4. Azimuthal Pitot probe scans with the $m=45$ plasma cone tip at $\mathrm{x}=17.8 \mathrm{~cm}(7 \mathrm{in})$ and $R e_{x}=1.75 \times 10^{6}$. The FFT is applied to the white area of the Pitot probe data series. $\mathrm{P}_{0}=172.4 \mathrm{kPa}(25 \mathrm{psi})$

in the latter. Their general similarity is to be expected since the $m=45$ case was meant to be a "controlled baseline", meaning it was intended to excite wave numbers that were in the most amplified range. However in addition to accomplishing that, it also showed a preference to $m=45$, that is a strong indication that the cross-flow instability was receptive to the plasma actuator array wave number.

In contrast to the $m=45$ case, the $m=68$ case was designed to draw energy away from the most amplified wave numbers, into the higher sub-critical wave number. At the most upstream location shown in Figure 5.6, the spectra of the azimuthal pressure distribution for the plasma-on condition shows a small increase in energy at $m=68$. The amplitude is still smaller than the peak near $m=55$ that is in the band of most-amplified wave numbers based on linear theory. However by the next streamwise location shown in Figure 5.7, the most dominant peak in the wave number spectrum is very close to $m=68$. The amplitude of this peak 

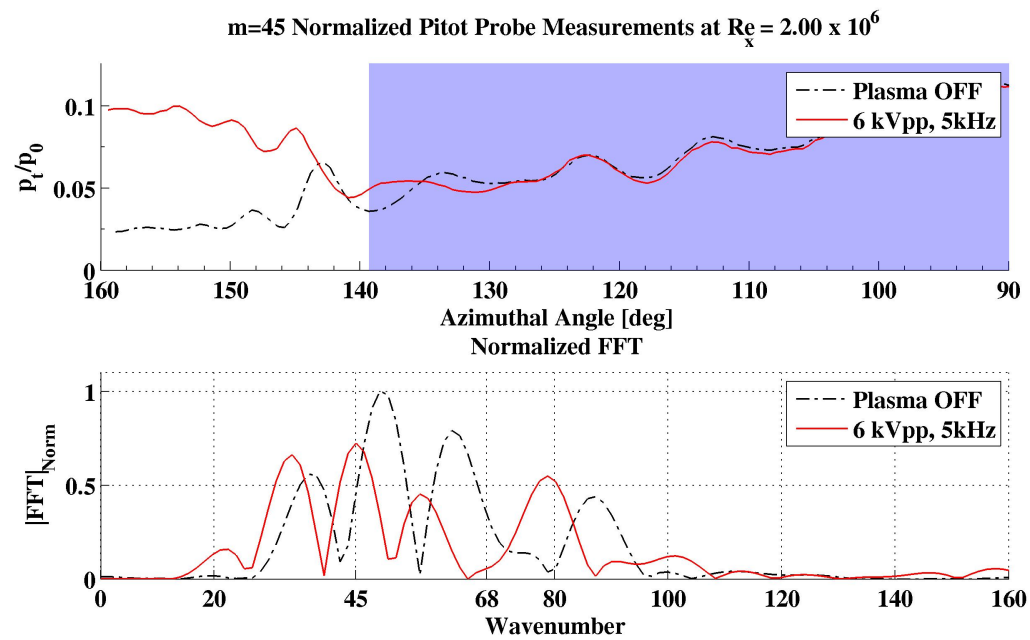

Figure 5.5. Azimuthal Pitot probe scans with the $m=45$ plasma cone tip at $\mathrm{x}=20.3 \mathrm{~cm}(8 \mathrm{in})$ and $R e_{x}=2 \times 10^{6}$. The FFT is applied to the white area of the Pitot probe data series. $\mathrm{P}_{0}=172.4 \mathrm{kPa}(25 \mathrm{psi})$

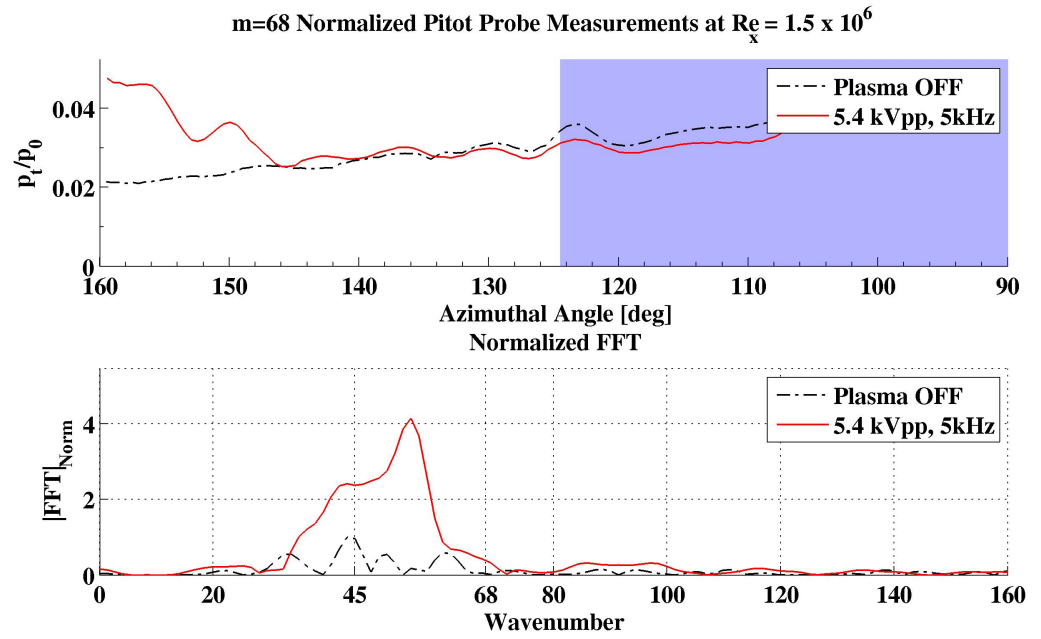

Figure 5.6. Azimuthal Pitot probe scans with the $m=68$ plasma cone tip at $\mathrm{x}=15.2 \mathrm{~cm}(6 \mathrm{in})$ and $R e_{x}=1.5 \times 10^{6}$. The FFT is applied to the white area of the Pitot probe data series. $\mathrm{P}_{0}=172.4 \mathrm{kPa}(25 \mathrm{psi})$ 

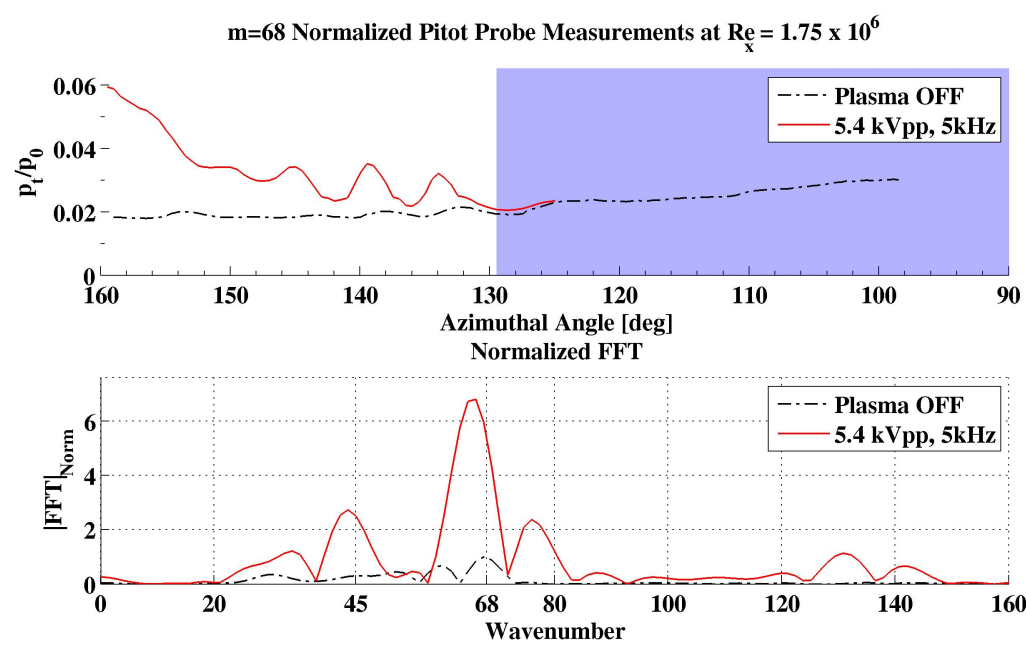

Figure 5.7. Azimuthal Pitot probe scans with the $m=68$ plasma cone tip at $\mathrm{x}=17.8 \mathrm{~cm}(7 \mathrm{in})$ and $R e_{x}=1.75 \times 10^{6}$. The FFT is applied to the white area of the Pitot probe data series. $\mathrm{P}_{0}=172.4 \mathrm{kPa}(25 \mathrm{psi})$

is seven times that of the baseline, plasma-off condition. Finally at the farthest downstream location, shown in Figure 5.8, the wave number spectrum still shows that significant energy is at $m=68$, however the predominant wave numbers have shifted to higher, and lower wave numbers that are outside of the linear most amplified range. A similar spectrum was observed in the analysis of the surface flow visualization and azimuthal pressure scans with the $m=68$ dimples.

The results in Figure 5.3 through 5.8 clearly confirm that the plasma actuator array performed like the passive patterned roughness. Specifically it was able to put energy into a specific azimuthal wave number of stationary cross-flow modes. 

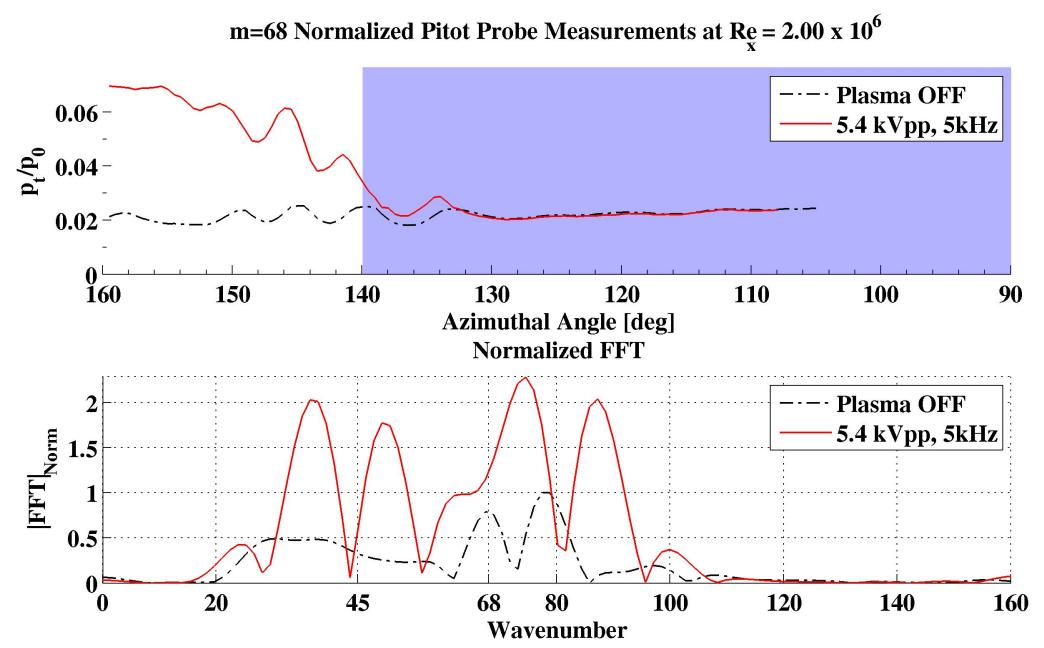

Figure 5.8. Azimuthal Pitot probe scans with the $m=68$ plasma cone tip at $\mathrm{x}=20.3 \mathrm{~cm}(8 \mathrm{in})$ and $R e_{x}=2 \times 10^{6}$. The FFT is applied to the white area of the Pitot probe data series. $\mathrm{P}_{0}=172.4 \mathrm{kPa}(25 \mathrm{psi})$

\subsection{Repeatability}

To demonstrate that the measured azimuthal pressure distributions with the plasma actuators operating were repeatable, numerous azimuthal Pitot probe scans were performed with a complete wind tunnel shutdown in between. The result of these repeatability experiments for the $m=68$ case is shown in Figure 5.9 .

It can be seen from the figure that the spatial fluctuations in the pressure, that is indicative of the mean flow distortion produced by the stationary crossflow modes, is highly repeatable over the leeward azimuthal angles, and different from the baseline case. The azimuthal angles where the plasma-on pressure distribution is not repeatable, is beyond the parting vortex line, in the blue shaded region. Within the affected azimuthal region, the stationary cross-flow modes were repeatable in amplitude, wavelength and azimuthal location. 


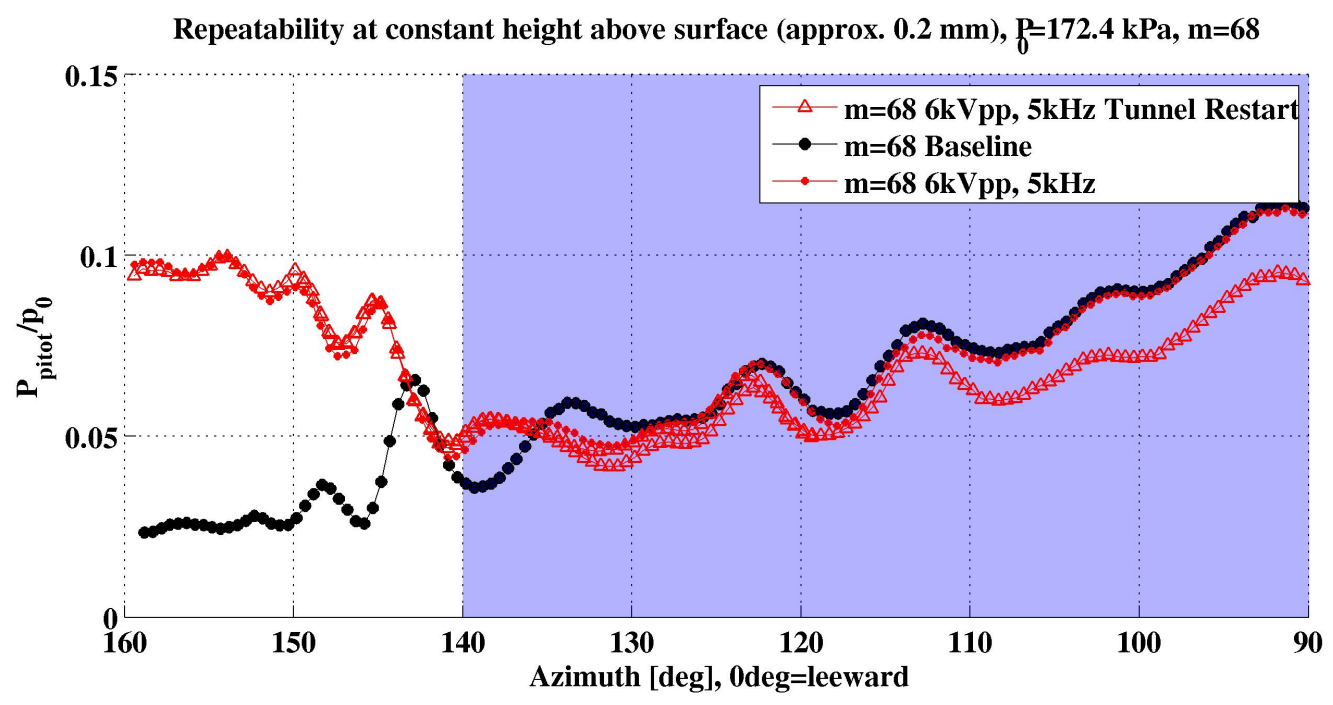

Figure 5.9. Repeated azimuthal pressure scans with plasma on before and after a wind tunnel restart. $m=68, \mathrm{P}_{0}=172.4 \mathrm{kPa}(25 \mathrm{psi})$, $T_{0}=311 \mathrm{~K}\left(100^{\circ} \mathrm{F}\right), R e_{x}=2 \times 10^{6}, \mathrm{x}=20.3 \mathrm{~cm}(8 \mathrm{in})$.

\subsection{Effect of Initial Amplitudes}

The effect of initial amplitude and the AC frequency of the DBD plasma actuator array on the formation of the stationary cross-flow vortices was investigated. The results are shown in Figure 5.10. This corresponds to $\mathrm{P}_{0}=172.4 \mathrm{kPa}$ (25 psia) and a streamwise location of $\mathrm{x}=20.3 \mathrm{~cm}$ ( 8 in) where $R e_{x}=2 \times 10^{6}$.

In one case, the voltage was increased for a constant AC frequency. In another case, the voltage was kept approximately constant, near $5 \mathrm{kVp}-\mathrm{p}$, and the $\mathrm{AC}$ frequency was increased from $1 \mathrm{kHz}$ to $6 \mathrm{kHz}$. We expect that the amplitude effect of the DBD plasma actuator array will increase linearly with AC frequency. We note that the amplitudes of the pressures in the azimuthal distributions increased in uniform increments between $\mathrm{AC}$ frequencies from $1 \mathrm{kHz}$ to $3 \mathrm{kHz}$. From $4 \mathrm{kHz}$ to $6 \mathrm{kHz}$, the amplitudes of the azimuthal pressure distributions stopped increasing, 
but rather saturated. These results indicate that at this streamwise locations, the initial amplitudes at the three lower AC frequencies were producing a linear response. The forcing levels at the higher AC frequencies were clearly at nonlinear levels.

Figure 5.10 also shows the effect of using two different $\mathrm{AC}$ voltages with the same $\mathrm{AC}$ frequency. In this case, the $\mathrm{AC}$ frequency was $5 \mathrm{kHz}$, and the voltages were $2.8 \mathrm{kVpp}$ and $4.3 \mathrm{kVpp}$. In contrast to a change in the $\mathrm{AC}$ frequency where the DBD actuator body force scales linearly, with a change in the AC amplitude, the body force scales as amplitude raised to a power. At the 0.1 bar static pressure in the experiment, the body force is proportional to $\mathrm{V}_{A C}^{2.4}$ [59]. Based on this, a change in voltage from $2.8 \mathrm{kVpp}$ to $5.1 \mathrm{kVpp}$ caused an increase in the plasma actuator body force by a factor of approximately four. This is approximately 2.5 times the change in the body force caused by changing the $\mathrm{AC}$ frequency from $1 \mathrm{kHz}$ to $6 \mathrm{kHz}$ in the previous discussion.

Examining the result of changing the $\mathrm{AC}$ amplitude in Figure 5.10 reveals that at the lower amplitudes, the pressure distribution fell nearly perfectly on the data at $5.1 \mathrm{kVpp}$ and a $2 \mathrm{kHz}$ frequency. At the higher $\mathrm{AC}$ voltage, the pressure distribution falls on the data at the higher $\mathrm{AC}$ frequencies, where the response was nonlinearly saturated. 


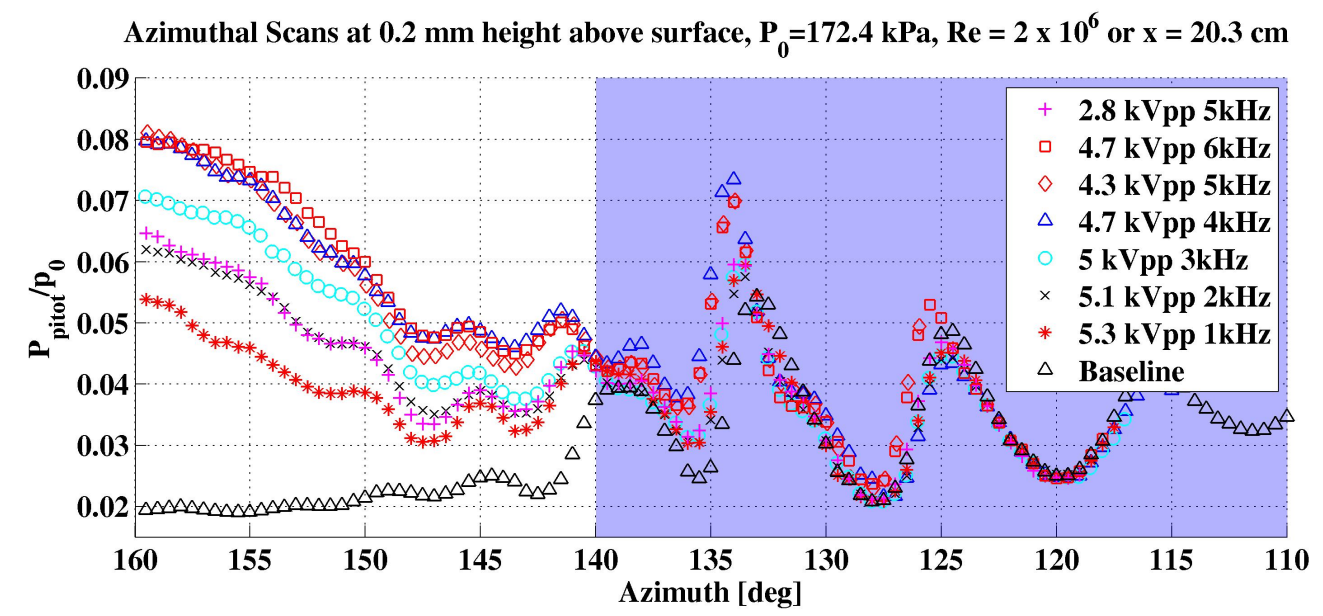

Figure 5.10. Repeated azimuthal pressure scans with AC driving frequencies from $1 \mathrm{kHz}$ to $6 \mathrm{kHz}$ and different voltages. $m=68$, $R e_{x}=2 \times 10^{6}, \mathrm{x}=20.3 \mathrm{~cm}(8 \mathrm{in}), \mathrm{P}_{0}=172.4 \mathrm{kPa}(25 \mathrm{psi}), T_{0}=311 \mathrm{~K}$ $\left(100^{\circ} \mathrm{F}\right)$ 


\section{CHAPTER 6}

\section{DISCUSSIONS AND CONCLUSIONS}

The main objective of this research was to demonstrate the delay of laminarturbulent transition of a boundary layer with cross-flow in a Mach 3.5 supersonic freestream flow by using patterned roughness. For this purpose, a right circular, $7^{\circ}$ half angle cone at a $4.2^{\circ}$ angle of attack was used in the NASA Mach 3.5 Supersonic Low Disturbance Tunnel (SLDT). In low disturbance environments, such as in free-flight or a low disturbance wind tunnel, cross-flow boundary layer transition is dominated by stationary cross-flow modes rather than the otherwise more amplified travelling cross-flow modes. The stationary cross-flow modes are highly receptive to three dimensional surface roughness at the Branch I neutral stability location. This characteristic can be used to excite specific wavelength stationary cross-flow modes with micron-sized patterned roughness. Saric et al. [45] demonstrated that the excitation of a weakly amplified stationary cross-flow mode inhibited the growth of the naturally more amplified stationary cross-flow modes. The excited mode suppressed the growth of the more amplified modes, and in so doing delayed turbulent transition. This subcritical wave number excitation has been demonstrated extensively in subsonic flow over swept wings. The object of this work was to demonstrate its effect in a supersonic flow.

The approach that was taken was to first document the effect of subcritical forcing using traditional micron-sized passive roughness arrays. The next approach 
was to replace the passive roughness with an array of DBD plasma actuators that could be actively controlled. The plasma actuator array was designed to produce streamwise vorticity that was similar to that produced by isolated roughness.

\subsection{Patterned Roughness}

Two cone tips were designed to excite the stationary cross-flow modes at critical and subcritical wavenumbers. Each cone tip surface had an azimuthal array of equidistant "dimples" that were approximately $30 \mu \mathrm{m}$ deep. The dimples were located slightly upstream of the Branch I linear stability neutral curve. The exact location was $\mathrm{x}=1.27 \mathrm{~cm}(0.5 \mathrm{in})$ from the cone tip. The dimple diameter and their spacing depended on the azimuthal mode number to be excited. In both cases, the ratio of the diameter to azimuthal wave length, $\mathrm{d} / \lambda$, was greater than 0.5 , following the recommendations of Saric [42].

Two other cone tips were designed for DBD plasma actuator arrays. The plasma actuator arrays consisted of two electrodes that were separated by the Macor wall of the cone tip. The Macor acted as the dielectric for the plasma actuator. The electrodes were vapor deposited onto the Macor to minimize any passive roughness effects caused by the presence of the exposed electrode on the surface of the Macor. With vapor deposition, the electrode thickness was only $60 \mathrm{~nm}$. This was far less than the rms surface roughness of the Macor which was $0.5 \mu \mathrm{m}$, and made it indistinguishable from the natural Macor surface. The exposed electrode edge was at the same axial location of the passive roughness. It was shaped like a comb, with the number of comb extensions ("fingers") corresponding to the azimuthal wavenumbers of $m=45$ and $m=68$ that represented the critical and subcritical forcing, respectively. The comb electrodes were verified in scaled-up 
PIV measurements, to produce pairs of counter rotating streamwise vortices. This was expected to be similar to the horseshoe vortex that results from flow over an isolated roughness element, including the roughness dimples.

\subsection{Receptivity to the Patterned Roughness}

\subsubsection{Flow Visualization}

The existence of stationary cross-flow vortices was initially shown with surface flow visualization. The cross-flow vortices were revealed as light and dark bands in the surface visualization coating. Spectral analysis of the bands was used to determine the wavenumber content of the cross-flow modes. The results for the smooth (baseline) cone tip confirmed the linear theory analysis of the predominant wave numbers. The results with the critical, $m=45$, forcing concentrated the energy in the most amplified band, and limited the range of higher wave numbers compared to the baseline case. The results with the subcritical, $m=68$, forcing focused energy at $m=68$, and suppressed energy in the most amplified band. In contrast to the critical forcing, the subcritical forcing also generated higher harmonic wave numbers, as well as a low wave number mode. Overall the flow visualization provided the first confirmation of the receptivity to the passive roughness.

\subsubsection{Pitot Probe Measurements}

Receptivity was further confirmed by directly measuring the mean flow distortion in the boundary layer that was produced by the stationary cross-flow modes. This was observed as a periodic increase and decrease in the total pressure near the wall that was the result of the periodic thickening and thinning of the bound- 
ary layer. The wave length of the pressure variations defined the azimuthal wave number.

A comparison between the measured cross-flow vortex wave numbers for the smooth (baseline) cone tip, and the critical, $m=45$, and subcritical, $m=68$, roughness tip was shown in Figures 4.5 to 4.9 . The spectral analysis of the azimuthal data series revealed two distinct results. If the complete azimuthal extent was used, the dominant wave numbers were more like the baseline case. However if the azimuthal portion on the leeward side of the cone was only considered, the wave number of the roughness was clearly visible in the spectra. This realization resulted in the defining of a "parting vortex line". The parting vortex line was found by comparing the experimental cross-flow vortex trajectories with those from simulations, which also followed relatively well the inviscid streamlines. By tracing these towards the tip of the cone, it was apparent that only the leeward azimuthal portions of the roughness array intersected with the cross-flow vortex trajectories. The demarcation line was what was defined as the parting vortex line. The portion of the flow over the cone that was on the windward side of the parting vortex line was not affected by the roughness array.

Receptivity to the plasma roughness was demonstrated by analyzing the azimuthal wave number spectrum on the leeward portion of the parting vortex trajectory as shown in Figures 5.3 to 5.8 . The existence of the parting vortex line was further validated by comparing the plasma-on and plasma-off cases in Figure 5.1 and 5.2. In these cases, the amplitude, azimuthal location and azimuthal wave number of the stationary cross-flow modes were completely repeatable on the leeward side of the parting vortex line. This was not the case on the windward side of the parting vortex line. 
The receptivity of the stationary cross-flow modes was equally good for both the passive (dimple) roughness and the plasma roughness. This is evident by comparing the results for the $m=68$ subcritical roughness that were shown in Figure 4.14 and 5.9 . In contrast to the smooth cone tip for the passive roughness, or the plasma-off case with the plasma roughness, the $m=68$ wavenumber was clearly repeatable from one wind tunnel run to the next, for the passive roughness, and with the plasma actuator on.

A comparison of the wave number spectrum between the $m=68$ passive roughness and plasma roughness showed them to be very similar. However it appeared that the spectral peak at $m=68$ was larger compared to the baseline with the plasma roughness than with the passive roughness compared to its baseline. The plasma actuator amplitude for this comparison was shown to be large enough so that at the downstream locations, the mean flow distortion was saturated. We had no control on the passive roughness amplitude, so that in a relative sense, its initial amplitude effect might have been smaller than that of the plasma roughness. If so, that might explain the relatively larger response of the $m=68$ cross-flow mode to the plasma roughness. An alternate explanation is that the surface of the cone tip for the plasma actuator was smoother than that of the cone tip used with the passive roughness. The passive roughness cone tip was made of Torlon which visibly had a rougher surface than the Macor tips used for the plasma actuator. The roughness surface would undoubtedly put energy in a broad range of wave numbers, making the patterned dimples less effective. 


\subsection{Laminar-Turbulent Transition Effect}

The onset of transition was estimated from the mean flow properties of the boundary layer. For that purpose, the Pitot probe was placed close to the cone surface to simulate a Preston tube measurement. The challenge with the spatially fixed stationary cross-flow vortices was that they introduced a strong mean flow distortion that made it difficult to distinguish an increase in shear stress caused by turbulent transition, to that of the mean flow distortion caused by stationary cross-flow modes. To overcome this problem, the Preston tube measurements were performed while moving along the path of a stationary cross-flow vortex. In that case, a sudden increase in the Preston tube pressure indicated an increase in the wall shear that signaled the onset of laminar-turbulent transition. This method showed that the transition Reynolds number with subcritical passive roughness increased by 25 to $50 \%$. A second method based on the disappearance of the coherent mean flow distortion caused by the stationary cross-flow modes, indicated a $50 \%$ increase in the transition Reynolds number.

A wavenumber spectrum analysis of the azimuthal Preston probe measurements indicated that the turbulent transition process was comparable to that observed in Saric's subsonic stationary cross-flow mode dominated transition experiments [4]. The measurements with the plasma roughness were performed at the lower stagnation pressure where natural transition did not occur on the cone. As a result, we were unable to measure the effect of the plasma roughness on the transition location. However, all of the measurements indicated that the stationary cross-flow modes were as, or more, receptive to the subcritical plasma roughness than to the passive roughness. Therefore we expect that the transition Reynolds number would have been increased by an amount similar to that of the 
passive roughness.

Although the fundamental effect of the active and passive excitation of the stationary cross-flow instability was found to be similar, there were two differences in the overall boundary layer response that were observed. One major difference was an increase in the local total pressure towards the leeward side of the cone when the plasma roughness was turned on. It appeared that the local high pressure region originated on the leeward side and then spread towards the windward side further downstream. If the increase in the local total pressure was interpreted as the onset of turbulent transition, it is unlikely this was due to excitation of stationary cross-flow modes since the $\mathrm{N}$-factors on the extreme leeward side, $\theta=180^{\circ}$, were quite small.

A second explanation is that the $\mathrm{AC}$ component of the plasma actuator might have excited a travelling instability that would amplify in the thicker boundary layer that developed on the leeward side of the cone, at $\theta=180^{\circ}$. The most logical instability to exist there is a Tollmien-Schlichting (TS) instability.

As indicated by Matlis [34], the physical frequency of the most amplified TS mode on the same cone at zero incidence, with the same flow conditions of $\mathrm{P}_{0}=172.4 \mathrm{kPa}(25 \mathrm{psi})$ and $\mathrm{T}_{0}=311 \mathrm{~K}\left(100^{\circ} \mathrm{F}\right)$, are from 20 to $30 \mathrm{kHz}$. Since the most unstable TS frequencies scale with the momentum thickness of the boundary layer, there is a possibility that the unstable frequencies were in fact in the range of the $\mathrm{AC}$ driving frequency, which was $5 \mathrm{kHz}$. As an approximation for the frequency scaling, the boundary layer thickness of Matlis' cone was compared to the boundary layer thickness of the cone at a $4.2^{\circ}$ angle of attack. The boundary layer thickness at a zero angle of attack was approximately $0.76 \mathrm{~mm}(0.03 \mathrm{in})$ at an axial location of $20 \mathrm{~cm}$ (7.9 in). Based on Balakumar's simulations [3] the bound- 
ary layer thickness on the cone at an angle of attack at the same axial location, was between $1.78 \mathrm{~mm}(0.07 \mathrm{in})$ and $3.04 \mathrm{~mm}$ (0.12 in) for azimuthal angles between $\theta=170^{\circ}$ and $\theta=180^{\circ}$. This gives a boundary layer thicknesses ratio of between 2.3 and 4. Based on this scaling, the most amplified TS frequencies towards the leeward ray would be between $5 \mathrm{kHz}$ and $8 \mathrm{kHz}$. Therefore it is possible that the plasma actuator excited TS waves on the leeward ray of the cone, and that resulted in the measured increase in the local total pressure.

The second main difference between the passive and active roughness was the magnitude of the azimuthal mean flow distortion associated with stationary cross-flow modes on the windward side of the parting vortex line. As discussed, cross-flow modes on the windward side of the parting vortex line were not influenced by the roughness array because the path of the mean streamlines did not intersect the location of the roughness. As a result, any stationary cross-flow modes that appeared on the windward side of the parting vortex line were the result of random roughness. One source of random roughness that was different between the passive and plasma roughness cases was the cone tip. The cone tip was 1.7 in long which meant that the downstream part of the tip would influence a larger windward azimuthal region. The surface of the Torlon cone tip was noticeable rougher than that of the Macor tip. Therefore that rougher surface could have been the nucleus of the stationary cross-flow modes with larger amplitudes that occurred with the Torlon tip. The smoother Macor tip minimized this effect, and as a result, it was much easier to denote the parting vortex line with the plasma roughness than with the passive roughness. Future experiments will more carefully take note of the passive surface roughness. 


\section{CHAPTER 7}

\section{RECOMMENDATIONS FOR FUTURE WORK}

\subsection{Experimental Work}

One of the main challenges in the design of the roughness arrays was the size and shape of the cone tip surface. It was extremely difficult to manufacture the plasma actuator array on such a confined space. Standard MEMS techniques were not directly applicable because of the surface curvature. It would have been very helpful to move the location of Branch I of the stationary cross-flow instability further downstream by either operating at lower unit Reynolds numbers, or by decreasing the cone angle of attack. Moving Branch I downstream would have allowed more space on which build the actuator array. This would have made it possible to design the plasma roughness as circular plasma dots or as streamwise plasma vortex generators, but without the additional azimuthal plasma edge that occurred in the present design. However, a reduced angle of incidence would have reduced the amplification rate of the cross-flow mode, and increased the amplification rate of the TS mode. This would need to be analyzed in a stability analysis of the boundary layer flow to possibly find a more optimum angle of attack.

Another possibility of improving the accessibility of the model for active plasma roughness control would be to consider of a different geometry. One possible model is a finite width wedge with conical sides [62], such as NASA's HyBolt model. An 
oblique attached shock forms at the leading edge and blends into outboard regions of conical flow centered on each corner apex. The center 2-D region of the wedge has a higher surface pressure than the adjoining conical flow regions, so that a mean cross-flow develops. Branch I of the stationary cross-flow instability occurs on the flat slope of the wedge. The most amplified wavelengths are in the range of millimeters instead of micrometers as was the case of the previously used cone. The advantage is that the cross-flow instability mainly occurs on a flat surface, which makes the development of the plasma roughness considerably easier.

As mentioned in the Introduction, the future application of the demonstrated control of stationary cross-flow modes is intended for supersonic aircraft with highly swept wings. Therefore, a swept wing model would be a highly relevant geometry to study the delay of turbulent transition with subcritically spaced plasma roughness elements. The geometry of the swept wing should be designed so that the location of Branch I is not located in a region with high surface curvature. In addition, the wavelength of the stationary cross-flow mode should be large enough to be easily excited with an array of millimeter-sized plasma roughness elements. These design recommendations would make the fabrication process considerably easier. Besides insuring the existence of a stationary cross-flow instability, other instability modes have to be avoided. These include an attachment line instability at the leading edge, a streamwise pressure gradient driven TS-wave, or a Görtler instability if there are concave surfaces. The viscous attachment line instability might be receptive to the non-stationary effects such as produced by the $\mathrm{AC}$ plasma driving frequency. It is therefore even more important to place the DBD plasma actuator array downstream of the leading edge region. If the model is intended to be run at adiabatic conditions, a thin, thermally conductive wall should 
be used. If laminar-turbulent transition should be detected with thermal techniques such as thermal paint or thermocouples, a model skin with high thermal conductivity will most likely make it more difficult to obtain sharp temperature gradients.

The spatial limitations that were experienced in the present experiment are of no concern for the application for full-scale swept wings. The wavelengths of the most amplified stationary cross-flow modes will be in the range of millimeters, which does not pose any problem for the DBD plasma roughness. The dielectric strength of the insulating material is also not a concern since the voltages needed to excite the disturbances are quite low. Therefore, thin dielectrics are sufficient. The question might arise if corrosion of the exposed electrode is a problem for longer operation in an outdoor environment. However, non-corrosive metals can be used. There is also the possibility of applying a thin protective coating on the actuator surface. This would shield the electrodes from environmental effects without overly weakening the electric field.

To control the roughness wave number, and forcing location, separately addressable plasma groups would need to be designed. This would allow the plasma roughness to adapt to different flight conditions.

Separately addressable arrays of plasma actuators might also be interesting for academic research, especially if the interaction of stationary and travelling crossflow modes needs to be observed. Again, the stationary cross-flow instability is highly sensitive to surface roughness. If deployable dimples, bumps or vibrating ribbons are used to excite travelling cross-flow modes, the stationary cross-flow modes are also excited at the same time. A plasma actuator of the kind that was used in the present experiments is invisible to the stationary cross-flow instability 
when turned off. This makes it possible to excite a travelling mode separately.

\subsection{Numerical Work}

Besides the linear stability analysis on the base flow for the present cone experiment, Balakumar performed DNS simulations on the development of stationary cross-flow modes excited by an array of Gaussian shaped bumps. It would be very interesting to replace the physical roughness elements in the DNS simulation with the body force generated by the DBD plasma. The plasma body force model needs to be able to account for the effect of ambient pressure and temperature as well as the three dimensional shape of the electrode. It would be interesting to see if there is a non-stationary response of the flow to the $\mathrm{AC}$ driving frequency of the plasma actuator, and what the effect would be, especially in terms of the development of stationary cross-flow modes.

A numerically less expensive method might be chosen by using nonlinear parabolized stability equations (NPSE) similar to simulations that have been performed by Li et al. [31, 32]. 
APPENDIX A

UNCERTAINTY CALCULATIONS

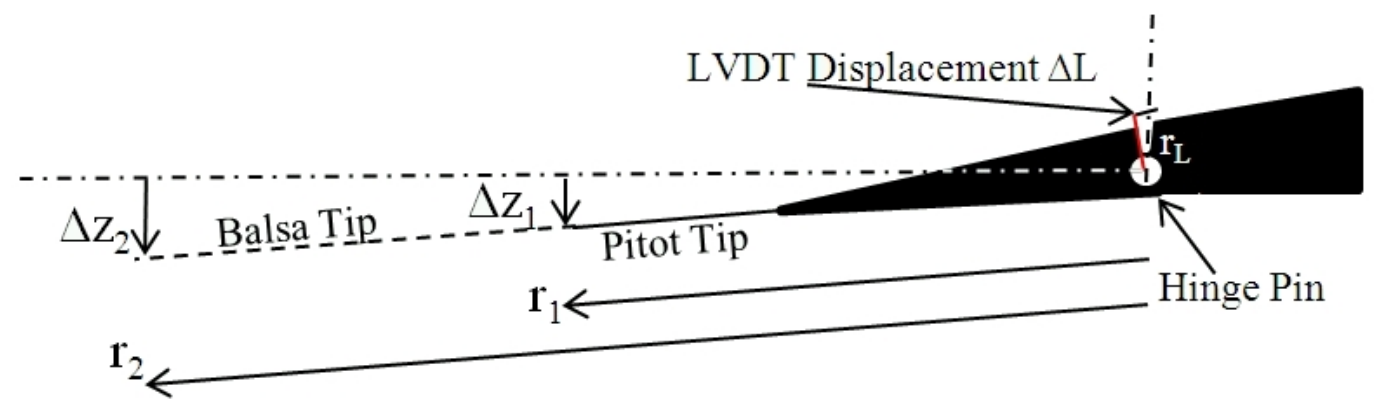

Figure A.1. Drawing of the Pitot tube, Balsa wood calibration piece and mounting wedge.

\section{A.1 LVDT Wall Normal Motion}

The displacement of the Balsa wood calibration arm $\Delta z_{2}$ can be calculated with

$$
\Delta z_{2}=\frac{r_{2}}{r_{L}} \Delta L
$$


and $r_{L}=10.55 \mathrm{~mm}$ and $r_{2}=249 \mathrm{~mm}$. The uncertainty in the measurements of the dimensions is 0.001 in or $0.025 \mathrm{~mm}$. The combined uncertainty in the LVDT displacement measurement based on the manufacturer's data is $2.8 \mu \mathrm{m}$. The range of displacement at the LVDT sensor is $\Delta L=1 \mathrm{~mm}$. In order to consider the influence of the measurement uncertainty of the geometric dimensions on the combined uncertainty $\mathrm{u}$ of the displacement of the Balsa wood tip , the change of $\Delta z_{2}$ with respect to $\Delta L, r_{L}$ and $r_{2}$ needs to be calculated for the maximum values of the range of motion. The combined uncertainty for the displacement $\Delta z_{2}$ of the tip of the Balsa calibration arm is then

$$
u\left(\Delta z_{2}\right)=\sqrt{\left(\frac{\partial \Delta z_{2}}{\partial \Delta L} \delta \Delta L\right)^{2}+\left(\frac{\partial \Delta z_{2}}{\partial r_{L}} \delta r_{L}\right)^{2}+\left(\frac{\partial \Delta z_{2}}{\partial r_{2}} \delta r_{2}\right)^{2}}
$$

- Finally the uncertainty in displacement at the Pitot tube tip can be calculated in the same manner as just done by knowing the relationship between the displacement of the Balsa wood tip and the Pitot tube tip.

$$
\Delta z_{1}=\frac{r_{1}}{r_{2}} z_{2}
$$

with $r_{1}=65 \mathrm{~mm}$ and $r_{2}=249 \mathrm{~mm}$. The measurement uncertainties of the lengths is again $0.001 \mathrm{in}$. The overall range of motion $z_{2}$ is $15 \mathrm{~mm}$. The final uncertainty for the Pitot tube tip displacement is then $23 \mu \mathrm{m}$ or $1.8 \%$ of the wall-normal motion range. 


\section{APPENDIX B}

\section{PRESSURE TRACE WAVENUMBER ANALYSIS}

The wavenumber analysis of the azimuthal pressure scans comparing the plasma on and off cases was done by applying a Fast Fourier Transform (FFT) to the high-pass filtered raw signal. The main challenge for the accurate identification of individual wavenumbers was the very short length of the data series. A typical data sample length ranged from 20 to 35 degrees, yielding a maximum of approximately two periods of a $m=45$ wave number. The data series length affects the wavenumber resolution and the ability to separate two neighboring wavenumbers based on their spectral magnitude. The sampling wavelength of the test data series was set to be $0.5^{\circ}$, which is the same sampling wave length as for the Pitot probe measurements. In order to increase the amount of displayed wave numbers in the computed spectrum, the raw signals were zero-padded to a total length of 512 samples.

A demonstration of the spectral analysis of a test data series is shown in the following figures. The test series, $y(x)$, as shown in Equation B.1 consists of the superposition of three sine-waves with $m=45, m=68$, and $m=6$ wavenumbers. These wavenumbers are representative of the expected wavenumbers in the Pitot probe measurements, namely the critical wavenumber, the subcritical wavenumber, and a slow variation of the overall pressure level. The amplitudes were chosen 
so that the dominant wavenumber was $m=68$.

$$
y(x)=A_{1} \sin \left(\frac{\pi}{180} m_{1}\right)+A_{2} \sin \left(\frac{\pi}{180} m_{2}\right)+A_{3} \sin \left(\frac{\pi}{180} m_{3}\right)
$$

with $m_{1}=68, m_{2}=45, m_{3}=6$, and $A_{1}=0.5, A_{2}=0.2$, and $A_{3}=2$. The raw test data series was then high-pass filtered at $m=25$ before the Fast Fourier Transform was applied. Figure B.1 illustrates the raw test series, and the high pass filtered test series on the left hand side, and the spectrum of the filtered series with and without a Hanning window on the right hand side. In all cases, $m=68$ is mapped into the frequency domain with the correct amplitude and wavenumber, whereas the $m=45$ wavenumber disappears in the side lobes of the $m=68$ wavenumber. This is an effect of the amplitude ambiguity which happens because the total sample period is not an integer multiple of the data series' contributory periods [17]. If the original data series is as short as in our case, it leads to a significant appearance of spilled frequencies close to the principal frequency. Windowing minimizes this effect, but the frequency resolution is still limited due to the very short original data series length.

One possibility to artificially increase the amount of data points would be to mirror the data series and apply the Fourier transform to the mirrored series. The result of the two-times mirrored original data series is shown in Figure B.2. An increase of the spectral resolution can be seen based on the sharper and narrower amplitude peaks of the spectra. Nonetheless, the problem of amplitude ambiguity is still present since the amplitude of the $m=45$ wavenumber does still not appear in the spectrum as distinct peak.

The amplitude ambiguity can be weakened by phase-shifting the mirrored data series so that the mirrored data overlaps with its unchanged counterpart over one 
Test Signal FFT
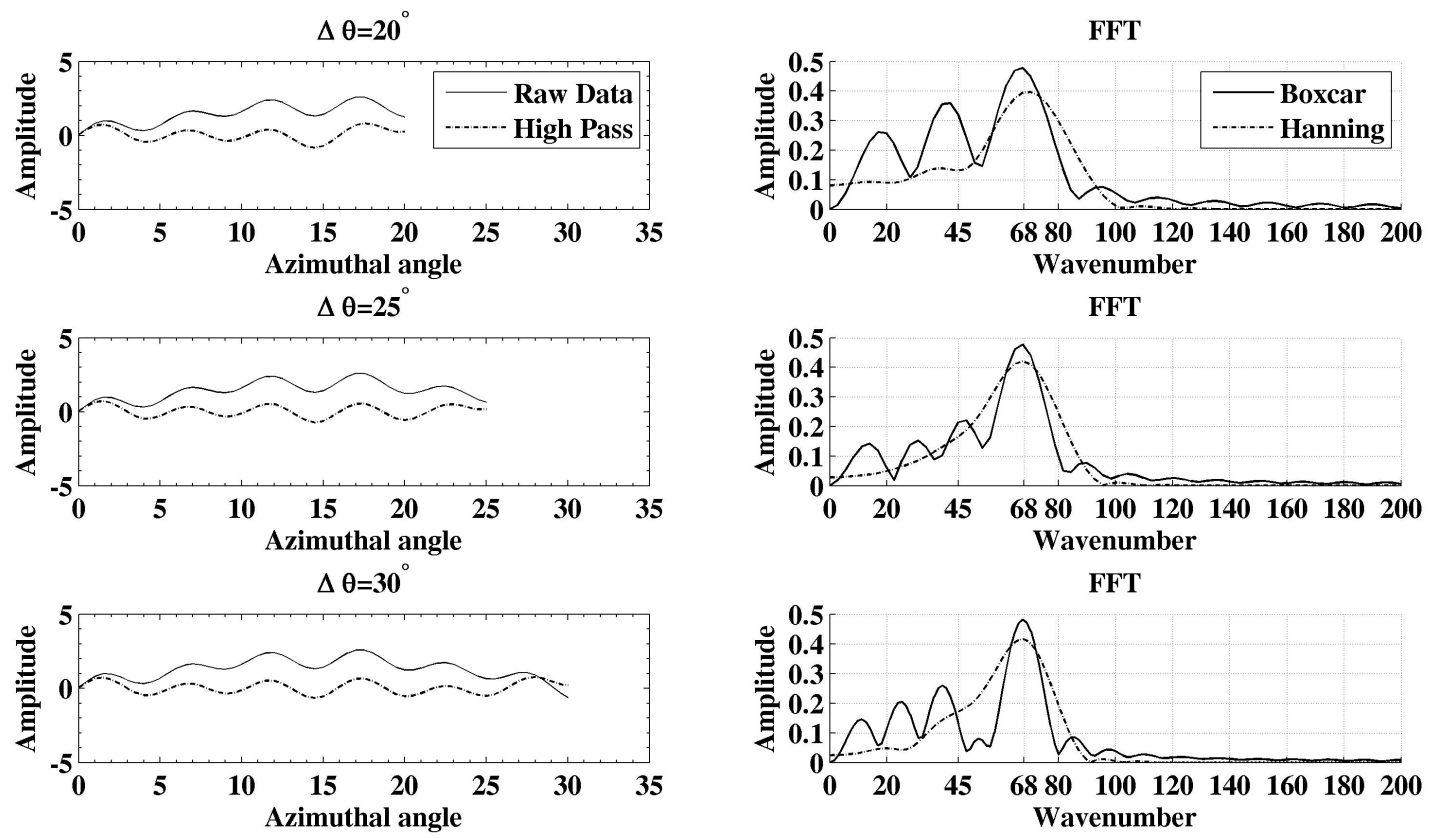

$\Delta \theta=35^{\circ}$
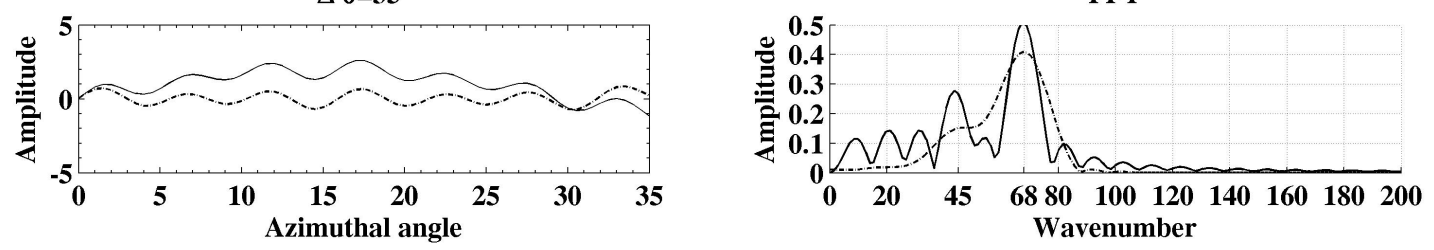

Figure B.1. FFTs of a representative sinusoidal test signal with varying sample lengths. 
Mirrored Test Signal FFT
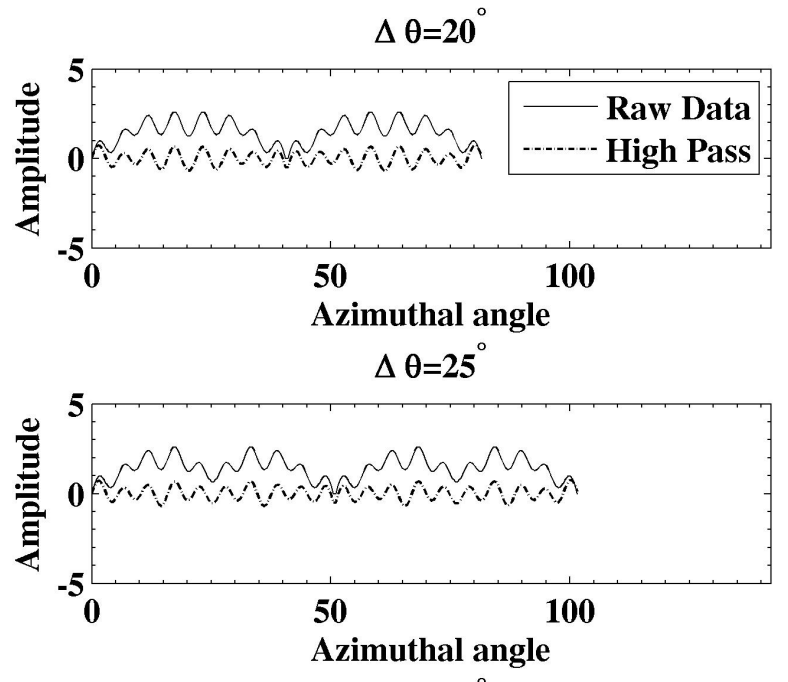

$\Delta \theta=30$

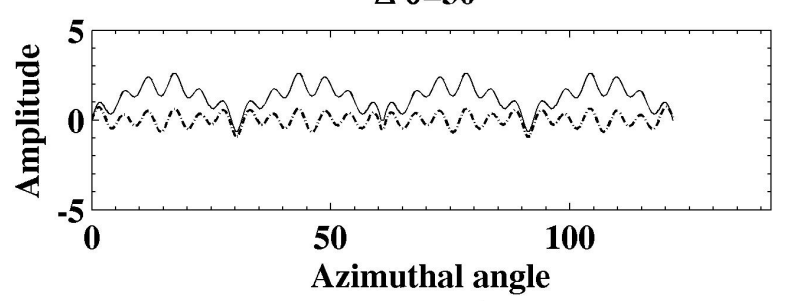

$\Delta \theta=35^{\circ}$

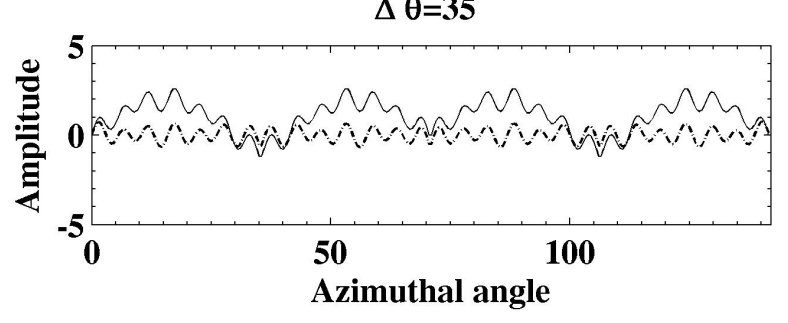

FFT

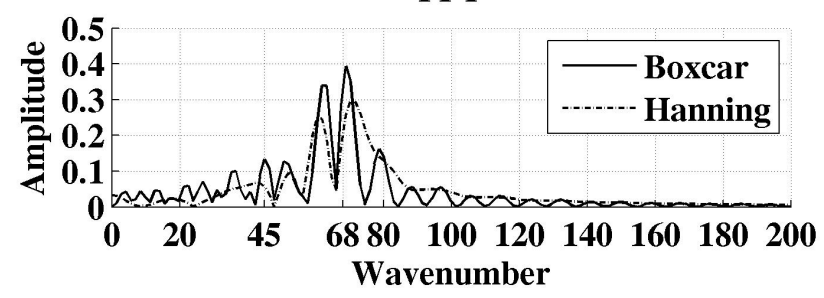

FFT

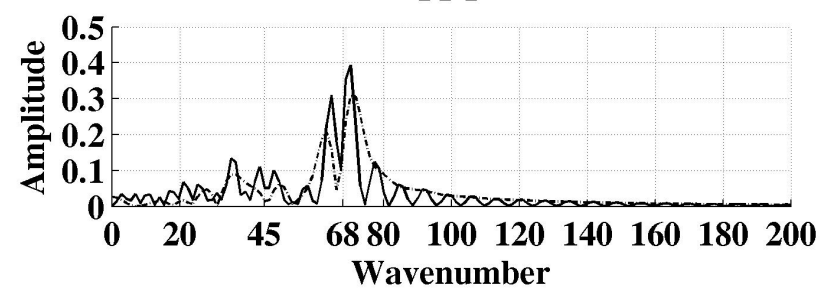

FFT

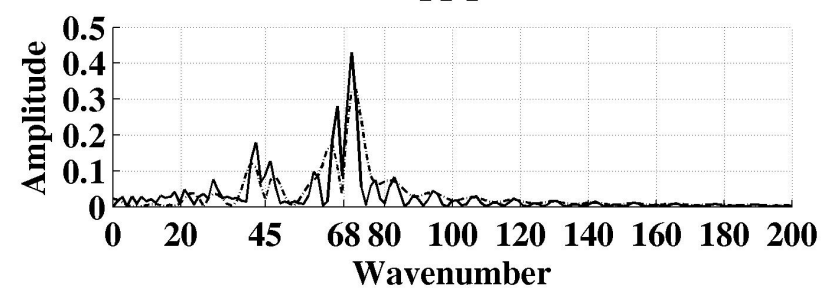

FFT

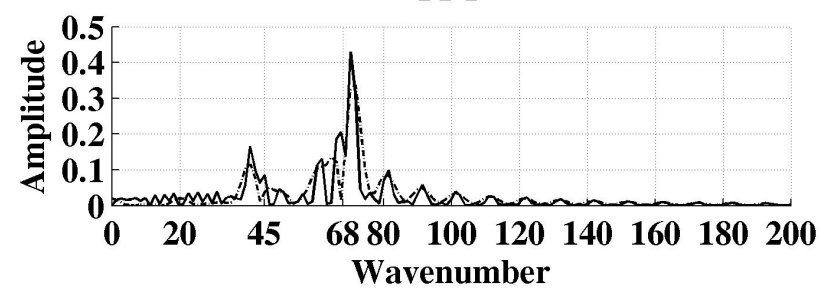

Figure B.2. The original data series from Figure B.1 was mirrored two times and then analyzed with a FFT. 
period. This removes most of the frequency spillage associated with step-like changes at the beginning and the end of the data series. Figure B.3 shows the effect of the mirrored and phase shifted signals. The $m=45$ wavenumber can now be clearly identified with a distinct peak for base sample lengths between $\Delta \theta=25^{\circ}$ and $\Delta \theta=35^{\circ}$.

The experimental Pitot probe pressure traces were processed in the same way as just shown for the test data series. At first, the raw data series was high-pass filtered at $m=25$. Then the data was mirrored and phase shifted in order to avoid steps in between the mirrored and original data series. The raw data, the filtered and mirrored data, as well as the amplitude spectra, for the plasma cone tip are shown in Figures B.4, B.5, B.6, B.7, B.8, and B.9. 
Mirrored Test Signal FFT
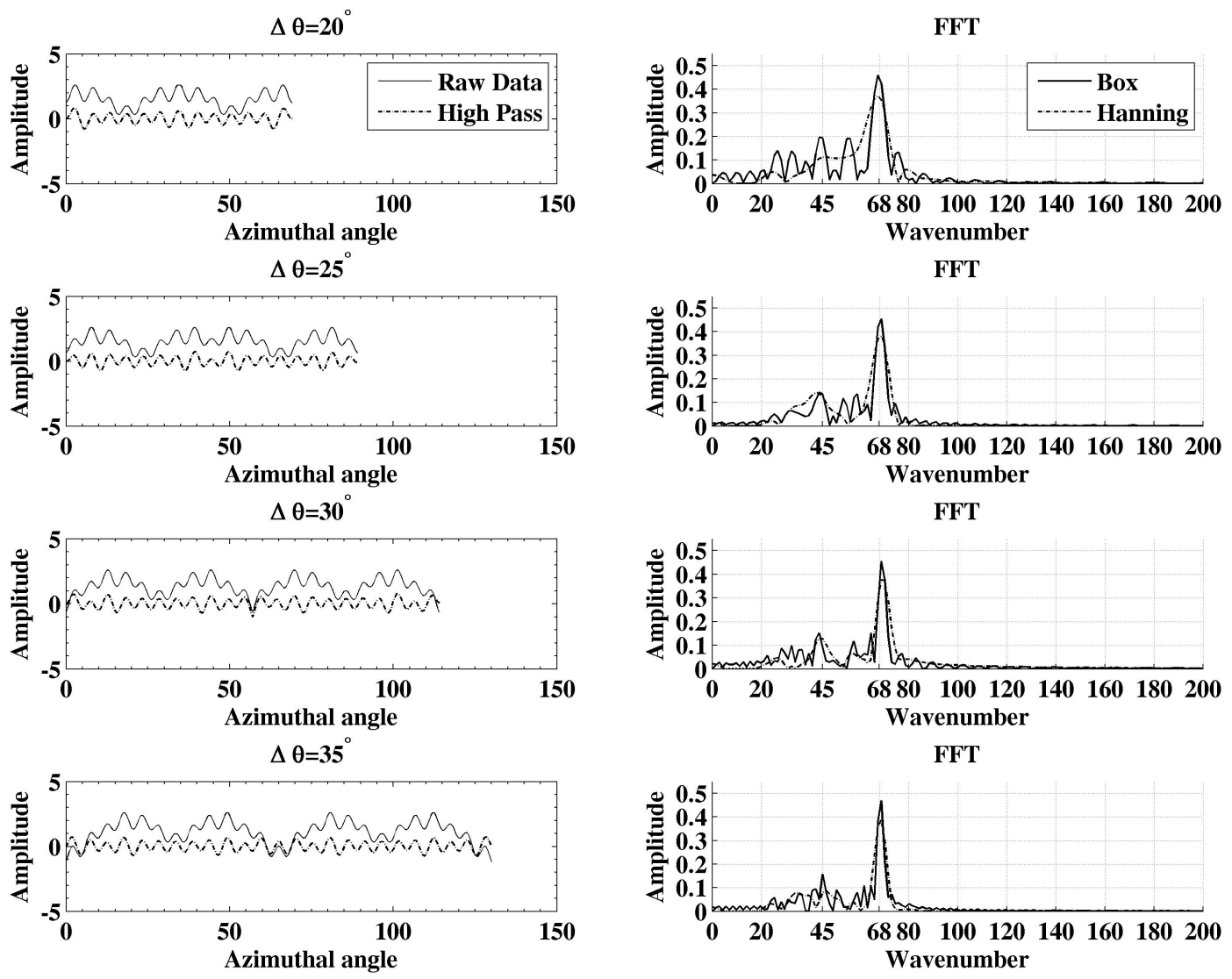

Figure B.3. Each mirrored data series was phase shifted in order to remove step changes at the end of the original signal. 

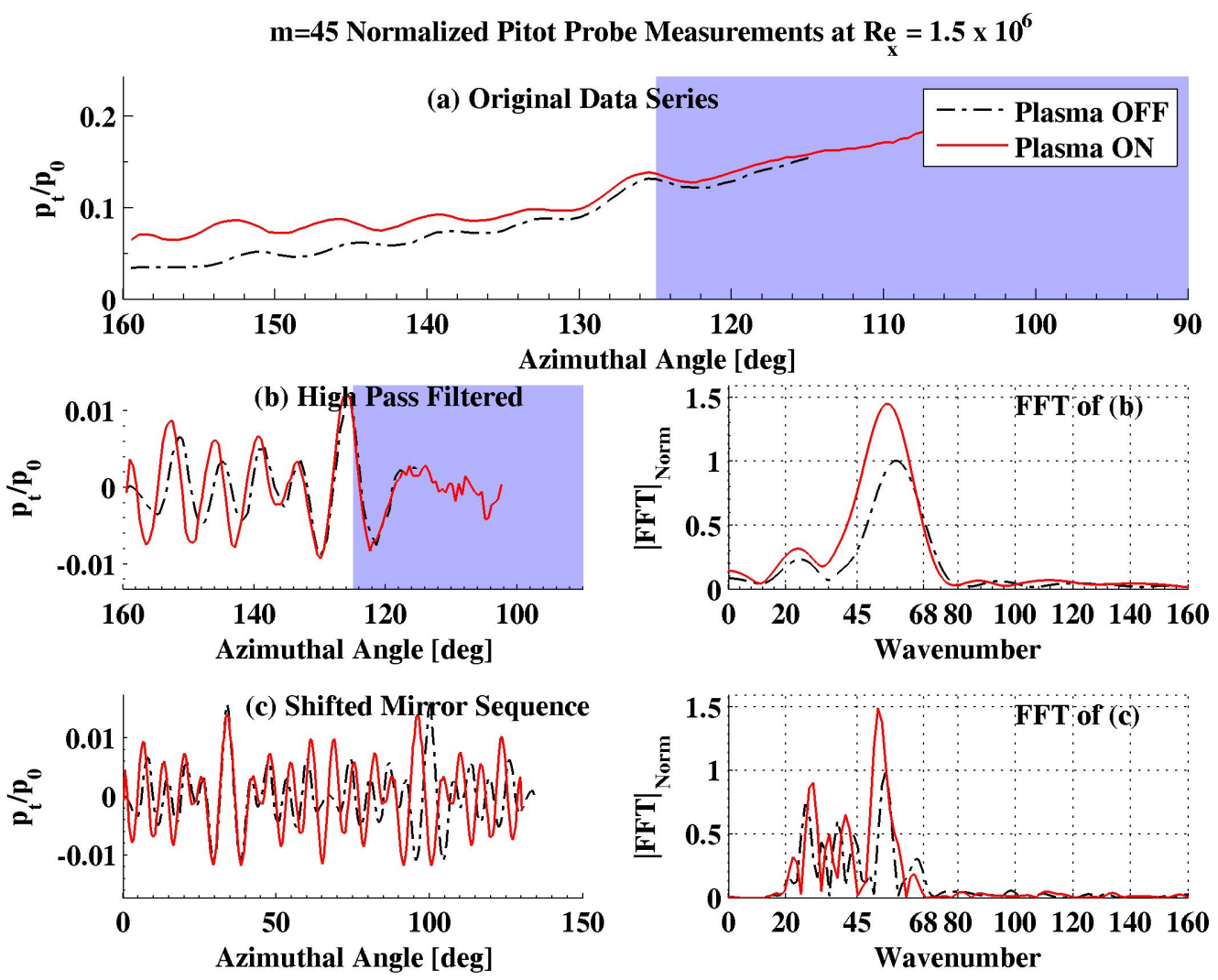

Figure B.4. $m=45, R e_{x}=1.5 \times 10^{6}, \mathrm{x}=12.7 \mathrm{~cm}$ (6 in). Summary of the original data series (a), the high pass filtered signal (b), the extended data series (b), and the respective FFTs. The blue regions mark the areas windward of the parting vortex trace. 

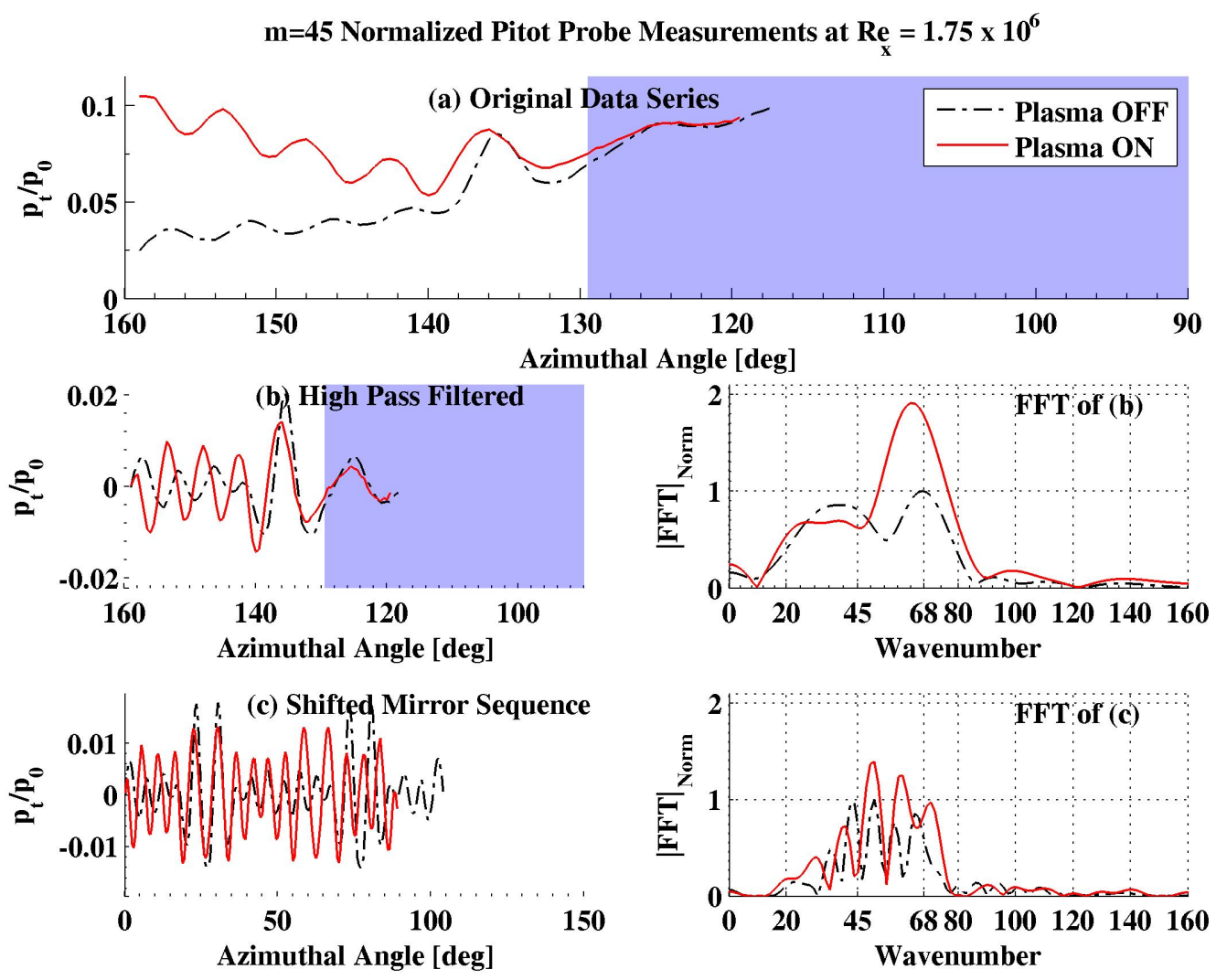

Figure B.5. $m=45, R e_{x}=1.75 \times 10^{6}, \mathrm{x}=17.8 \mathrm{~cm}(7 \mathrm{in}), \mathrm{P}_{0}=172.4 \mathrm{kPa}$ (25 psi). Summary of the original data series (a), the high pass filtered signal (b), the extended data series (b), and the respective FFTs. The

blue regions mark the areas windward of the parting vortex trace. 

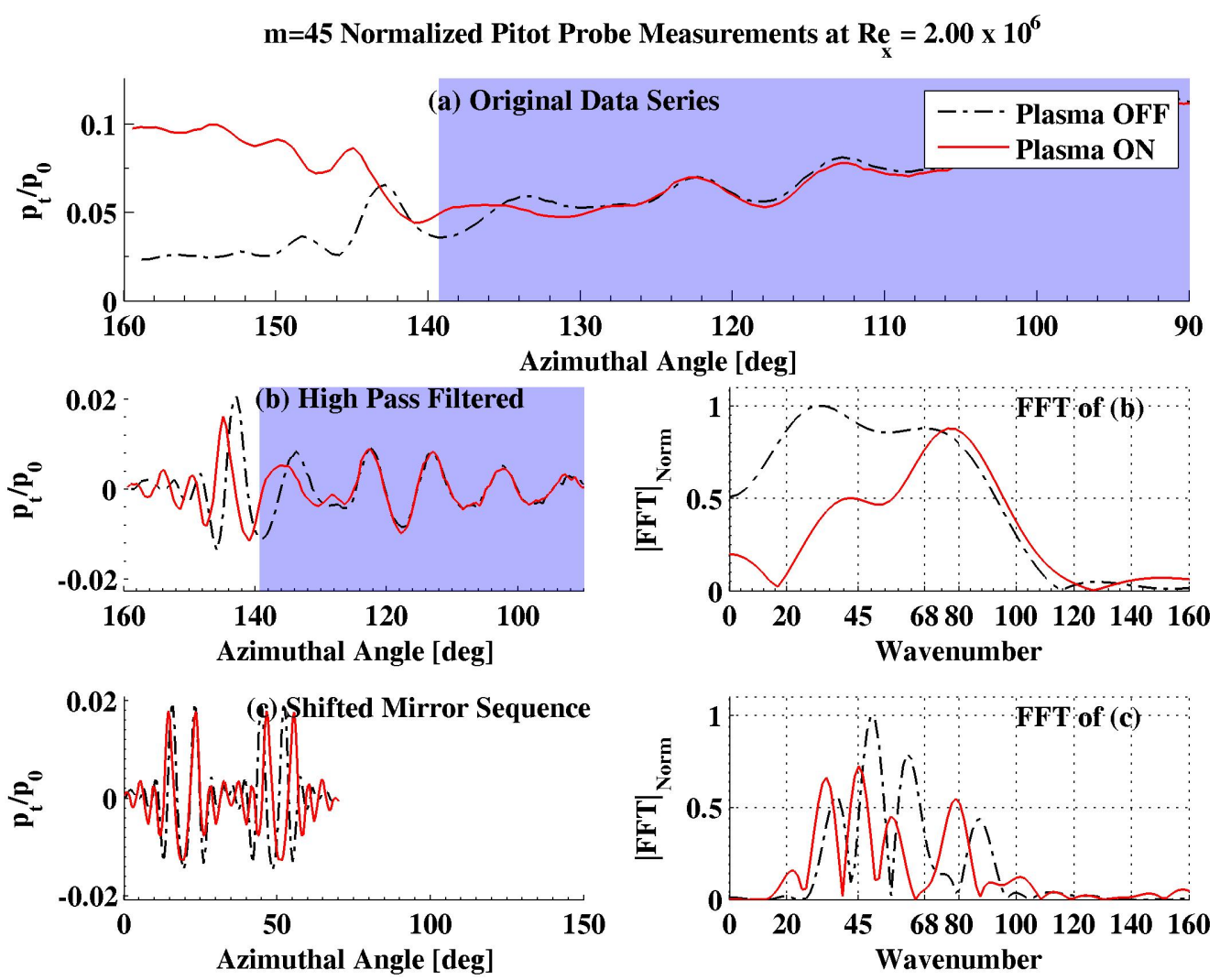

Figure B.6. $m=45, R e_{x}=2 \times 10^{6}, \mathrm{x}=20.3 \mathrm{~cm}(8 \mathrm{in}), \mathrm{P}_{0}=172.4 \mathrm{kPa}$ (25 psi). Summary of the original data series (a), the high pass filtered signal (b), the extended data series (b), and the respective FFTs. The

blue regions mark the areas windward of the parting vortex trace. 

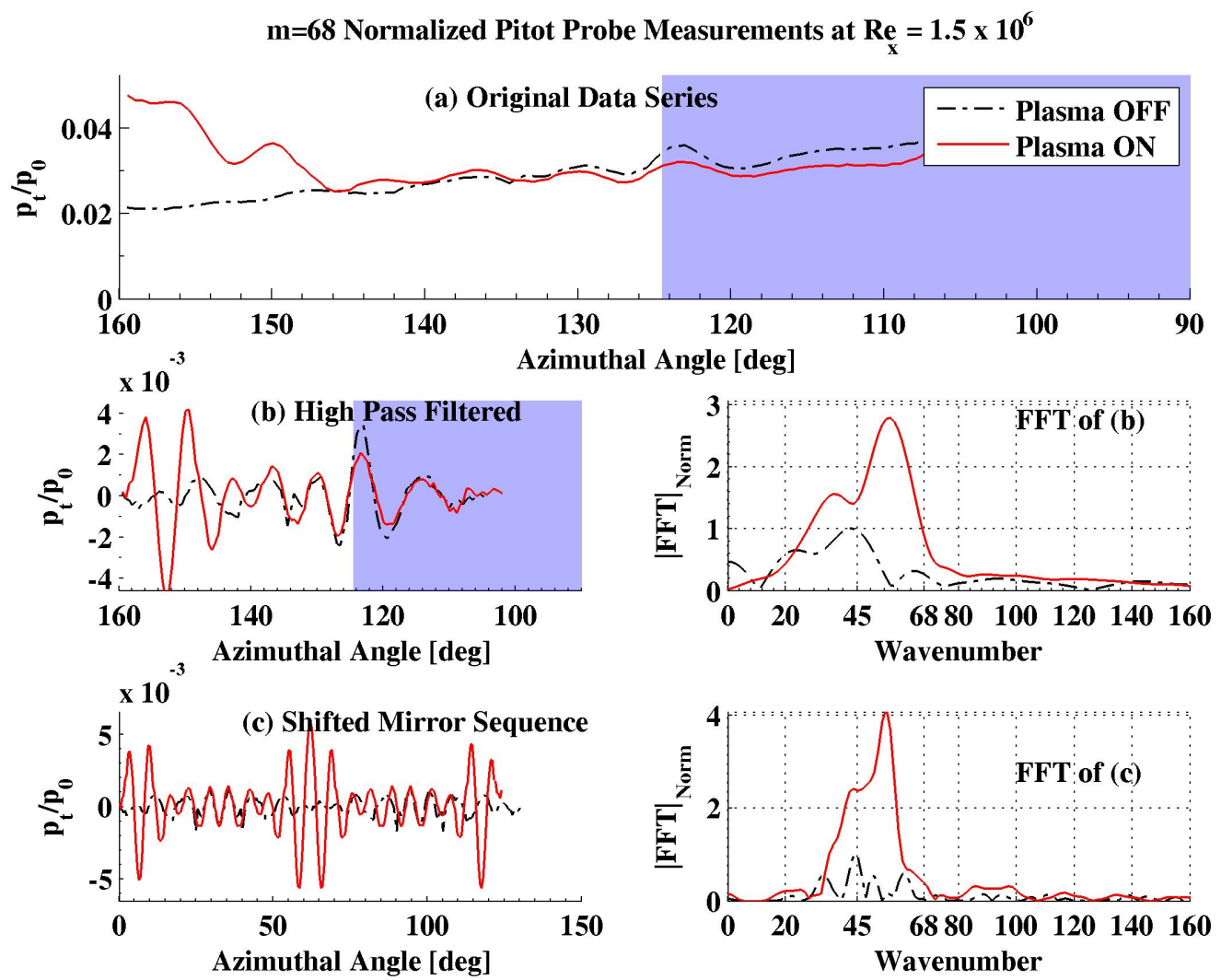

Figure B.7. $m=68, R e_{x}=1.5 \times 10^{6}, \mathrm{x}=12.7 \mathrm{~cm}(6 \mathrm{in}), \mathrm{P}_{0}=172.4 \mathrm{kPa}$ (25 psi). Summary of the original data series (a), the high pass filtered signal (b), the extended data series (b), and the respective FFTs. The

blue regions mark the areas windward of the parting vortex trace. 

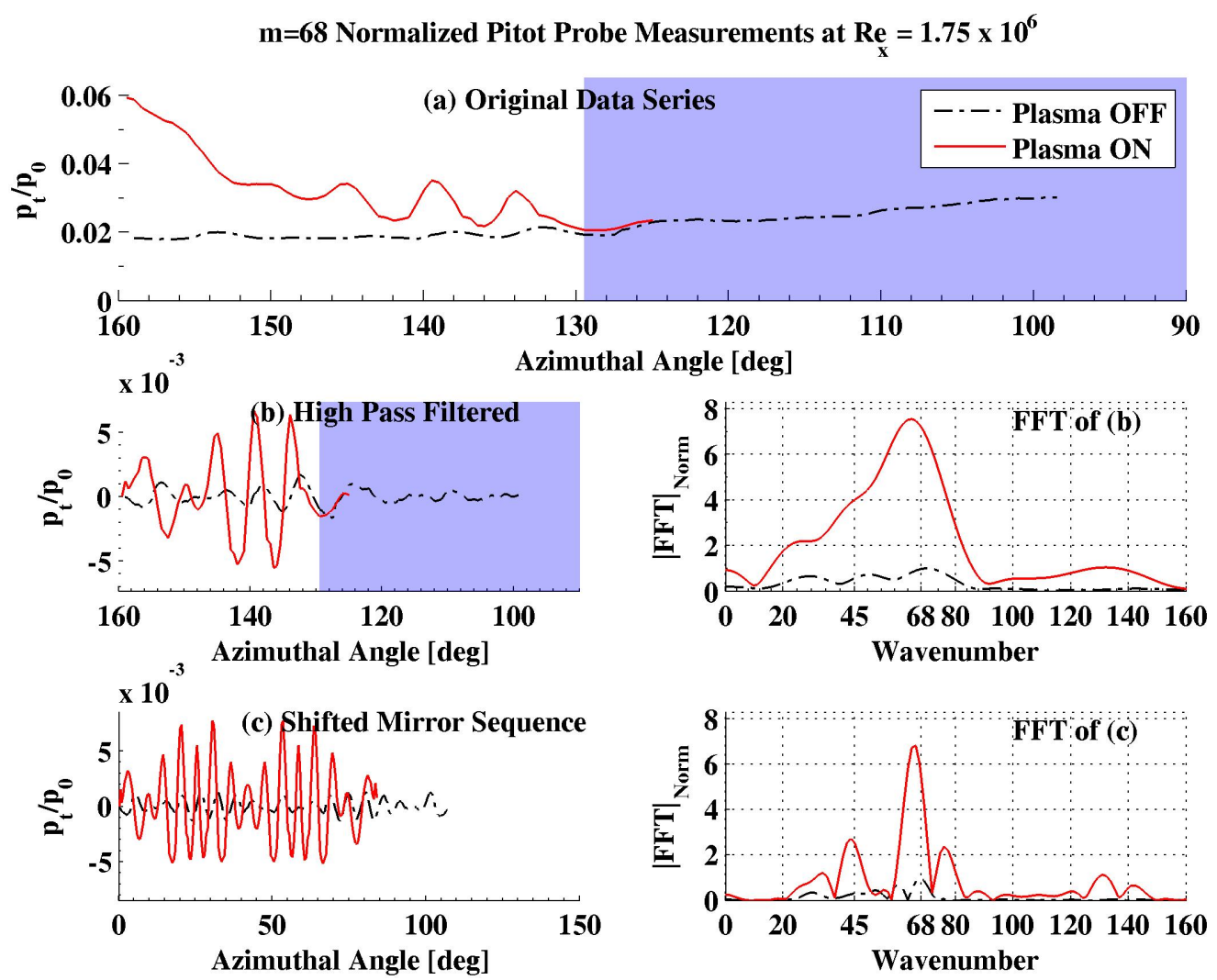

Figure B.8. $m=68, R e_{x}=1.75 \times 10^{6}, \mathrm{x}=17.8 \mathrm{~cm}(7 \mathrm{in}), \mathrm{P}_{0}=172.4 \mathrm{kPa}$ (25 psi). Summary of the original data series (a), the high pass filtered signal (b), the extended data series (b), and the respective FFTs. The

blue regions mark the areas windward of the parting vortex trace. 

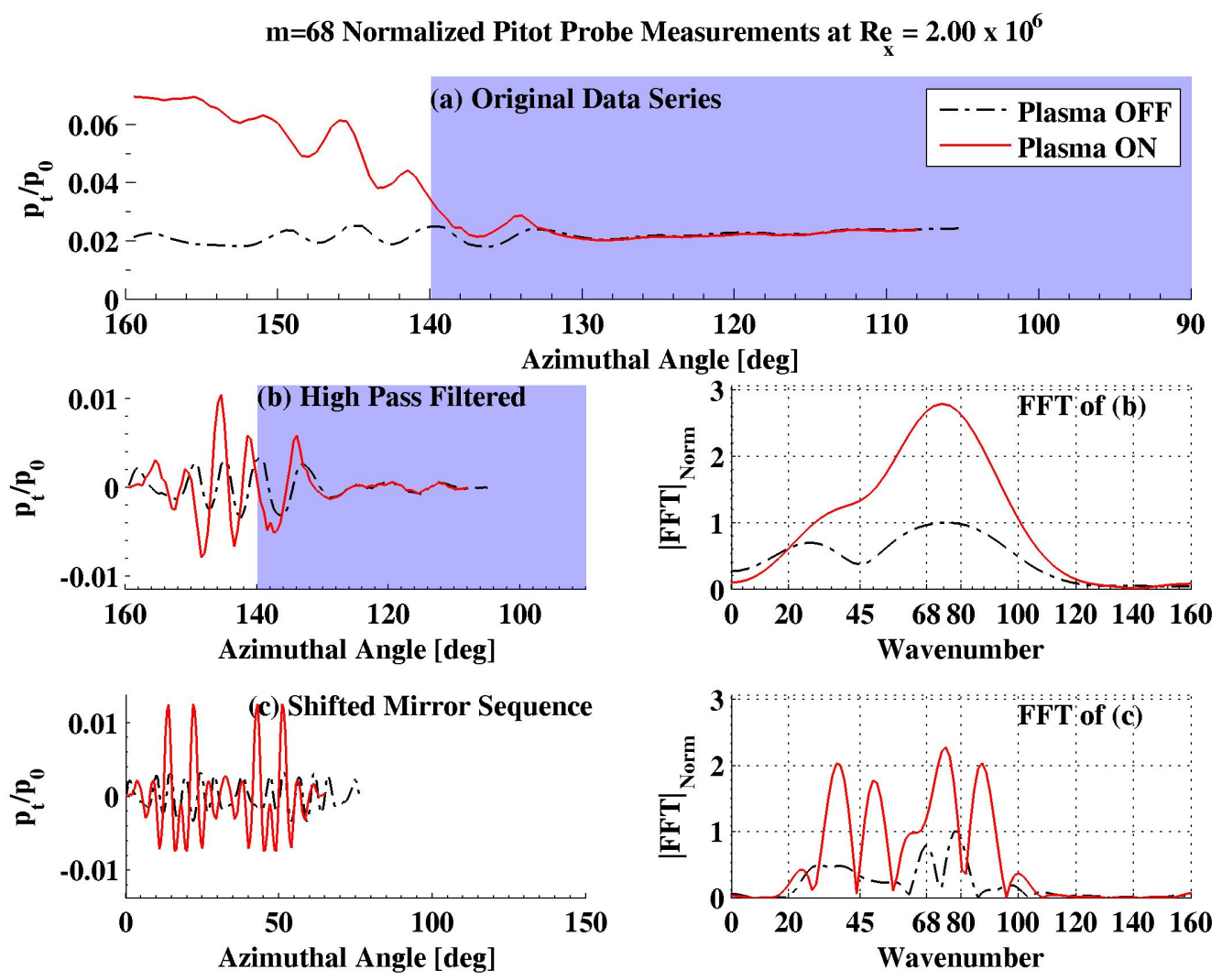

Figure B.9. $m=68, R e_{x}=2 \times 10^{6}, \mathrm{x}=20.3 \mathrm{~cm}(8 \mathrm{in}), \mathrm{P}_{0}=172.4 \mathrm{kPa}$ (25 psi). Summary of the original data series (a), the high pass filtered signal (b), the extended data series (b), and the respective FFTs. The

blue regions mark the areas windward of the parting vortex trace. 


\section{APPENDIX C}

\section{FAST RESPONSE PITOT PROBE AND HOT WIRE MEASUREMENTS}

\section{C.1 Pitot Probe Analysis}

For all of the boundary layer measurements, a Pitot tube with pressure lag times in the order of several seconds was used. The long pressure lag times were the result of the small Pitot probe diameter, the diameter of the Tygon ${ }^{1}$ tubing that connects the Pitot tube and the pressure transducer and the dead volume of the pressure transducer. The chosen Pitot probe diameter and length was already a compromise between the introduced Mach number error due to probe interference, spacial resolution, and lag time. It would be therefore desirable to use a Pitot probe with small diameter (smaller than $0.2 \times 0.1 \mathrm{~mm}$ ID x OD [34]) to maximize the spatial resolution and minimize flow interference while having a small pressure lag time of the measurement system. If the pressure lag time is small enough, even temporal data could be taken.

\section{C.1.1 Sinclair and Robins}

Sinclair and Robins [54] proposed a formula for the pressure lag time $t$ in dependence of the length $l_{e}$, diameter $d$, total measuring system volume $V$, the

\footnotetext{
${ }^{1}$ Saint-Gobain Performance Plastics, Akron, $\mathrm{OH} 44305,0.05$ in ID, $2 \mathrm{ft}$ length
} 
volume displacement caused by a displacement of the pressure transducer membrane $V_{d}$, the pressure inside the pressure transducer $p_{0}$, and the pressure at the orifice $p_{1}$. Their formula is based on fully developed Hagen-Poiseuille flow through a cylinder with no-slip wall conditions.

$$
t=\frac{128 \mu l_{e}}{\pi d^{4}}\left[\frac{V_{1}}{p_{1}} \ln \frac{\left(p_{0}-p_{1}\right)\left(p+p_{1}\right)}{\left(p-p_{1}\right)\left(p_{0}+p_{1}\right)}+\frac{3 V_{d}}{p_{0}-p_{1}} \ln \frac{p_{0}+p_{1}}{p+p_{1}}+\frac{V_{d}}{p_{0}-p_{1}} \ln \frac{p_{0}-p_{1}}{p-p_{1}}\right]
$$

The equation describes the time until the pressure $\mathrm{p}$ is reached with an applied pressure step from $p_{0}$ to $p_{1}$. If $\mathrm{p}$ is defined as $p=\frac{1}{\sqrt{2}} p_{1}$, the time $\mathrm{t}$ corresponds to the time constant $\tau$ of the system which defines the bandwidth at $f=1 / 2 \pi \tau$. One of the initial assumptions is the no-slip condition at the inner tube walls. In order to evaluate if this holds true for the expected tube diameters and ambient pressures, the Knudsen number needs to be calculated. The Knudsen number $K n$ is defined as the ratio between the molecular mean free path $l[27$ and a reference length $D$. The reference length is in our case chosen as the diameter of the Pitot tube which is the smallest geometrical dimension. $k_{B}=1.38065 \times 10^{-23}$ is the Boltzmann constant and $\sigma=3.711 \times 10^{-10}$ is the hard shell diameter of an air molecule [61]. $T_{0}$ and $p_{0}$ are the temperature and pressure at total conditions.

$$
K n=l / D=\frac{k_{B} T}{\sqrt{2} \sigma^{2} p_{0} d}
$$

The range of measured total pressures in the conducted experiments are in between $3 \mathrm{kPa}$ and $30 \mathrm{kPa}$. The corresponding Knudsen numbers for different Pitot tube diameters are shown in figure C.1. It can be seen that for smaller Pitot probe diameters and the lower range of pressures, the Knudsen number approaches 0.1 which means that a correction for wall slip needs to be applied. Therefore Sinclair 


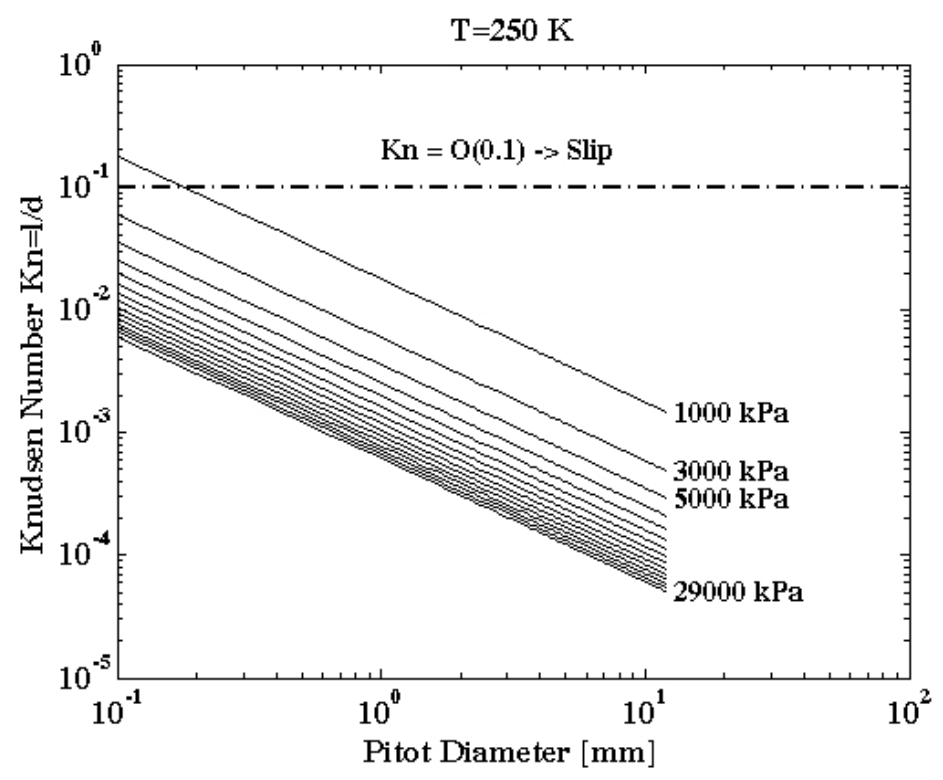

Figure C.1. Knudsen number for different total pressures and Pitot probe diameters

and Robin's equation is to be re-formulated considering wall slip. The derivation including wall slip in dependence of the mean free path of air molecules $l$ is done as shown in the corresponding derivation with no-slip conditions by Kennard [27] and shown in the following steps.

$$
u=-\frac{d p / d x}{4 \mu}\left(r_{0}^{2}-r^{2}+2 l r_{0}\right)
$$

Equation C.3 describes the fully developed velocity in a cylindrical pipe with the radius $r_{0}$ exposed to an axial pressure gradient $d p / d x$. The dynamic term for the mass inertia is set to zero which means that start-up effects are not considered. This is a simplification which means that the obtained lag times are most likely shorter than the real lag times. Therefore the results which will be obtained can be seen as the upper limit in terms of frequency response. Anyhow, a fully developed 
laminar flow will form in relatively short times [61], especially with small Pitot probe diameters which means that the steady flow approximation is valid for small probe diameters. If equation C.3 is multiplied by the density and then integrated over the cross section of the pipe, an expression for the mass flow $Q_{m}$ can be derived.

$$
Q_{m}=\frac{p}{R T} \int_{0}^{r_{0}} 2 \pi r u d r=-\frac{\pi}{8} \frac{r_{0}^{4} p}{\mu R T}\left(1+4 \frac{l}{r_{0}}\right) \frac{d p}{d x}
$$

The massflow is assumed to be the same over the length of the pipe $l_{e}$ which means that the mass flow can be integrated along $\mathrm{x}$ from 0 to $l_{e}$ and can be expressed as

$$
\left.Q_{m}=\frac{\pi}{256} \frac{d^{4} l_{e}}{\mu R T}\left(p(0)^{2}-p\left(l_{e}\right)^{2}\right)\right)\left(1+16 \frac{l}{d}\right)
$$

Now, the mass flow can be expressed as $R T \frac{d m}{d t}=p \frac{d V}{d t}+V \frac{d p}{d t}$. If the volume $V$ does not change within the Pitot probe and the attached transducer, the mass flow can be written as $Q_{m}=\frac{V}{R T} \frac{d p}{d t}$. This equation can then be combined with equation C.5 to get an expression for the dynamic change of the pressure at the transducer end of the Pitot probe in dependence of the pressure at the open Pitot probe end.

$$
\left.\frac{V}{R T} \frac{d p}{d t}=\frac{\pi}{256} \frac{d^{4} l_{e}}{\mu R T}\left(p(0)^{2}-p\left(l_{e}\right)^{2}\right)\right)\left(1+16 \frac{l}{d}\right)
$$

Similar to equation C.1, the variables are renamed according to their meanings. The pressure $p\left(l_{e}\right)=p_{1}$ is the pressure at the opening of the Pitot tube where the pressure is applied, whereas $p(0)=p$ is the pressure which is sensed at the transducer. The volume $V=V 1$ is the constant system volume. The variables in equation C.6 are then separated as shown in equation C.9 and integrated.

$$
\left.\frac{V_{1}}{R T} \frac{d p}{d t}=\frac{\pi}{256} \frac{d^{4} l_{e}}{\mu R T}\left(p^{2}-p_{1}^{2}\right)(1+16) \frac{l}{d}\right)
$$




$$
d t=\frac{256 \mu l_{e}}{\left.\pi d^{4}(1+16) \frac{l}{d}\right)}\left(\frac{V_{1}}{2 p_{1}} \ln \frac{p-p 1}{p+p 1}\right)
$$

The time t to reach the pressure $\mathrm{p}$ is then estimated by evaluating the definite integral at $t=0$ and $p=p_{0}$. The final equation for the time $\mathrm{t}$ is then

$$
t=\frac{128 \mu l_{e}}{\pi d^{4}(1+16 K n)}\left(\frac{V_{1}}{p_{1}} \ln \frac{(p 0-p 1)\left(p+p_{1}\right)}{(p-p 1)\left(p_{0}+p_{1}\right)}\right)
$$

The resulting equation with slip is exactly the same as with no-slip except for a correction factor based on the Knudsen number.

The bandwidth is shown in dependence of $l_{e} / d$ and for different ambient pressures under the assumption that $V_{d} /\left(p_{0}-p_{1}\right)$ is negligible. This means practically that the deformation of the membrane at the pressure transducer is negligible. This assumption is true e.g. for Piezo resistive pressure transducers. The values in figure C.2 are therefore the upper limit for the expected bandwidth of a Pitot

probe based high frequency response measurement system. At the lowest measured total pressures around $2000 \mathrm{kPa}$, a theoretical band width around $300 \mathrm{~Hz}$ could be expected with a Pitot probe of $0.1 \mathrm{~mm}$ diameter and $12 \mathrm{~mm}$ length. At atmospheric ambient pressure, that value goes up to $500 \mathrm{~Hz}$. If a Pitot probe with the same dimensions as in the current experiments is used, the ratio of $l_{e} / d=12 / 0.25=48$ which translates into a theoretical bandwidth between $2 \mathrm{kHz}$ at $1000 \mathrm{kPa}$ total pressure and approximately $4 \mathrm{kHz}$ at atmospheric pressure.

\section{C.1.2 Helmhotz Resonator}

Sinclair and Robin's approach describes the Pitot tube as first order system. Another approach can be taken by considering the inertia of the displaced air in the 


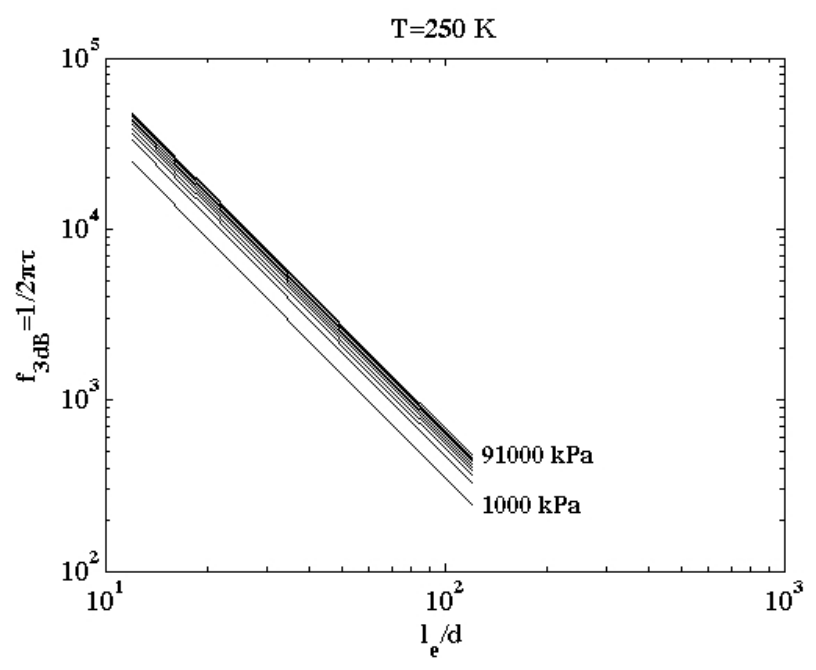

Figure C.2. Bandwidth of the Pitot tube in dependence of the length to diameter ratio and for different ambient total pressures. Data is corrected with Knudsen number as shown in equation C.9. Each line shows the bandwidth for different ambient pressures in $10000 \mathrm{kPa}$ steps

Pitot tube. This results in a second order system which is similar to the description of a Helmholtz resonator. The measurement system consists in this case of a cavity which holds the diaphragm of the pressure transducer and a tube that connects the cavity to the atmosphere. It is assumed that the air in the tube with length $\mathrm{L}$ and radius $\mathrm{r}$ is incompressible and displaces as one rigid column moving with an average velocity of $u$. This assumption is valid if the wavelength of the disturbance is larger than the length of the tube. The cavity acts as compressible spring, obeying the ideal gas law, $p=\rho R T$. If an external change in pressure $\Delta p_{i n}$ pushes air into the constant volume cavity, the pressure changes by an amount $\Delta p_{0}$. This change can be described as $\Delta p_{0}=\frac{\Delta m R T}{V}=\frac{\rho_{1} \pi r^{2} \Delta x R T}{V}=\frac{p_{1}}{V} \pi r^{2} \Delta x$. V is the constant volume of the cavity, $p_{1}$ is the total pressure at the Pitot probe and $\Delta x$ is the displacement of the air column with density $\rho_{1}$ inside the tube. The 
equation of motion is then formulated as follows

$$
\pi r^{2} L \rho_{1} \frac{\partial^{2} \Delta x}{\partial t^{2}}=\pi r^{2}\left(p_{1}+\Delta p_{i n}\right)-\frac{8 \pi L \mu}{1+8 K n} \frac{\partial \Delta x}{\partial t}-\pi r^{2}\left(p_{1}+\Delta p_{0}\right)
$$

$\mu$ is the dynamic viscosity of air. $\frac{8 \pi L \mu}{1+8 K n} \frac{\partial \Delta x}{\partial t}$ corresponds to the viscous damping of the system which is based on the wall friction with slip of the fully developed laminar Hagen-Poiseuille flow. The displacement $\Delta x$ of the air column is then substituted by the functional relationship between $\Delta p_{0}$ and $\Delta x$. It finally yields

$$
\Delta p_{i n}=\frac{L V \rho_{1}}{p_{1} \pi r^{2}} \frac{\partial^{2} \Delta p_{0}}{\partial t^{2}}+\frac{8 L V \mu}{(1+8 K n) r^{4} \pi p_{1}} \frac{\partial \Delta p_{0}}{\partial t}+\Delta p_{0}
$$

The transfer function is therefore

$$
H(s)=\frac{\Delta p_{0}(s)}{\Delta p_{i n}(s)}=\frac{\frac{p_{1} \pi r^{2}}{L V \rho_{1}}}{s^{2}+\frac{8 \mu}{(1+8 K n) r^{2} \rho_{1}} s+\frac{p_{1} \pi r^{2}}{L V \rho_{1}}}=\frac{\omega_{n}^{2}}{s^{2}+2 \zeta \omega_{n} s+\omega_{n}^{2}}
$$

with the natural frequency $\omega_{n}=r \sqrt{\frac{p_{1} \pi}{V L \rho_{1}}}=r \sqrt{\frac{R T \pi}{L V}}$ and a damping factor of $\zeta=\frac{4 \mu}{(1+8 K n) r^{3}} \sqrt{\frac{L V \rho_{1}}{\pi p_{1}}}=\frac{4 \mu}{1+8 K n) r^{3}} \sqrt{\frac{L V}{\pi R T}}$. The Amplitude of the transfer function in dependence of the frequency input is

$$
|H(s)|=\frac{\left|\Delta p_{0}(s)\right|}{\left|\Delta p_{i n}(s)\right|}=\frac{1}{\sqrt{\left(1-a^{2}\right)^{2}+(2 \zeta a)^{2}}}
$$

with $a=\frac{\omega}{\omega_{n}}$. The natural frequency of the system can be calculated by assuming again a length of the Pitot tube of $0.012 \mathrm{~m}$. The diameter is varied from $0.0001 \mathrm{~m}$ to $0.0002 \mathrm{~m}$ (which would correspond to half the diameter of the $1.7 \mathrm{~mm}$ diameter Kulite) as well as the temperature T. The cavity volume is assumed to be based on a cylindrical volume with $1.7 \mathrm{~mm}$ diameter and $2 \mathrm{~mm}$ length. The cavity can be made smaller but in order to assume that an incompressible column of air moves 


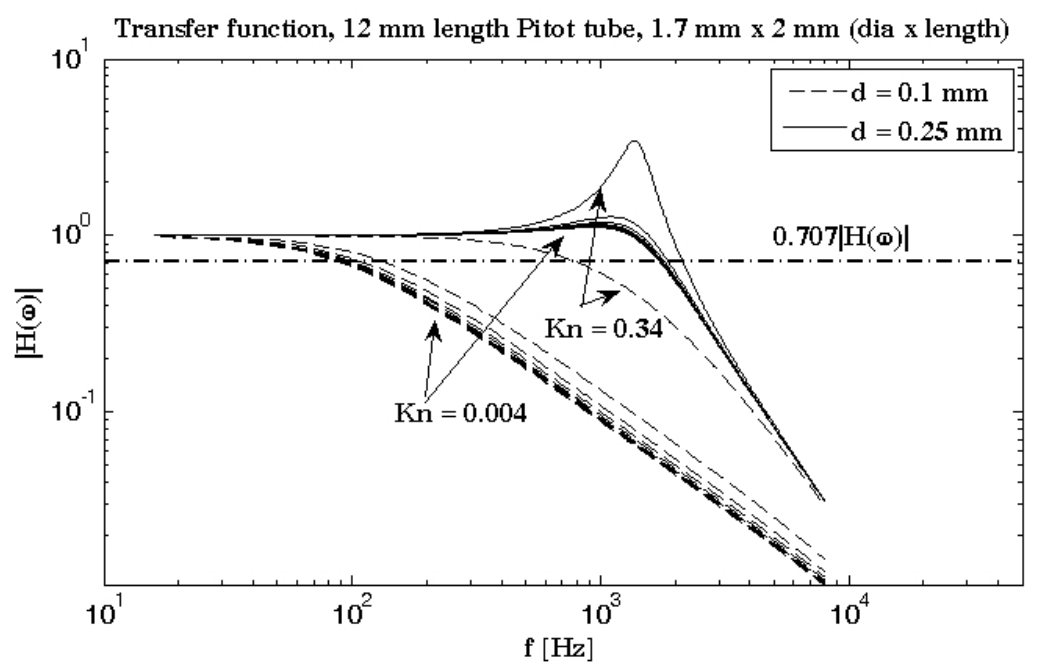

Figure C.3. Transfer function for $0.1 \mathrm{~mm}$ and $0.25 \mathrm{~mm}$ diameter Pitot tubes at different Knudsen numbers for $T_{0}=300 \mathrm{~K}$

inside the tube, the cavity volume has to be much larger than the volume of air in the Pitot tube itself. The transfer function for Knudsen numbers corresponding to pressures between $1 \mathrm{kPa}$ to $100 \mathrm{kPa}$ and a temperature of $300 \mathrm{~K}$ is shown in figure C.3. The system behaves for the smaller Pitot tube diameter like an overdamped system. The bandwidth is in this case lower than the corresponding case with the analysis not including inertia. If the Pitot tube diameter is doubled, the system responds like an underdamped system with the largest amplitude response at the natural frequency. Viscous damping is highly dependent on the radius of the Pitot tube whereas the eigenfrequency of the system is defined by the size of the cavity. The basic assumptions made in this approach are that the air column inside the Pitot probe behaves like an incompressible fluid which is true if the wavelength of the measured disturbance is much larger than the Pitot tube length, that the processes are isothermal, and that the volume of the air contained in the Pitot tube is small compared to the transducer cavity. Finally a fully developed laminar 
Hagen-Poiseuille flow is asserted when the fluid column moves inside the Pitot tube including a correction factor for large Knudsen numbers.

\section{C.1.3 Damped Wave Equation}

One more idea to get a more accurate calculation of the frequency response of a Pitot tube system is solving the damped wave equation. In this approach the fluid column inside the Pitot tube is assumed to displace with its radially averaged speed. Different from the shown models, the pressure gradient inside the tube is not assumed to be constant but depends on its axial location. No assumptions need to be made about the wavelength of the disturbance and an arbitrary cavity volume can be chosen. The derivations of the wave equation are briefly shown and discussed whereas the concrete solution of the problem is left to a future date.

At first the force balance on a cylindrical volume with the initial length $d x$ and volume $d V=\pi r^{2} d x$ is done. There are two pressures, $p(x)$ and $p(x+d x)$ which assert a force normally to the corresponding surfaces $\pi r^{2}$. Additionally there is the viscous drag which acts on the surface of the infinitesimal fluid column at its radial surface $F_{w}=\tau_{w} 2 \pi r d x$. The force balance yields

$$
\begin{aligned}
\rho_{0} \pi r_{0}^{2} d x \frac{\partial^{2} u}{\partial t} & =-\frac{\partial p}{\partial x} d x \pi r_{0}^{2}-\tau_{w} 2 \pi r_{0} d x \\
\rho_{0} \frac{\partial^{2} u}{\partial t^{2}} & =-\frac{\partial p}{\partial x}-\tau_{w} \frac{2}{r_{0}}
\end{aligned}
$$

whereas $u(x)$ is the displacement of the fluid disc at the location $x$ and $\rho_{0}$ is the density at the initial state. The mass $d m=\rho d V=\rho_{0} d V$ is constant because the boundaries of the force balance stay with the fluid particles and no flow of mass across them occurs. $r_{0}$ is the radius of the Pitot tube. The pressure $p(x)$ and the displacement $u(x)$ can be linked through the ideal gas law $p=\frac{m}{V} R T$. 


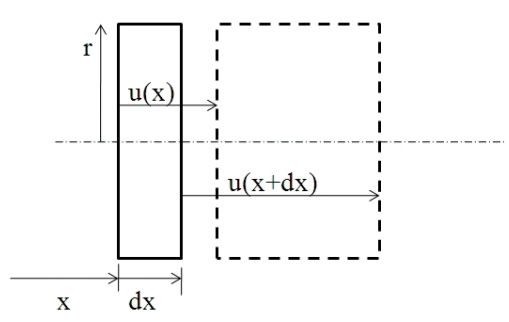

Figure C.4. Displacement of an infinitesimally thin disc of fluid inside the Pitot tube.

The pressure in the fluid disc is based on the volume and its mass. The mass is constant and is defined by its initial value whereas the new volume is based on the displacements $u(x)$ and $u(x+d x)$.

$$
p=\frac{\rho_{0} \pi r_{0}^{2} d x}{\pi r_{0}^{2}(u(x+d x)+d x-u(x))} R T=\frac{\rho_{0} d x}{\left(d x+\frac{\partial u}{\partial x} d x\right)} R T=\frac{\rho_{0} R T}{1+\epsilon}=\frac{p_{0}}{1+\epsilon}
$$

$\epsilon=\frac{\partial u}{\partial x}$ and $p_{0}$ is the total pressure at the initial state of the Pitot tube. In the next step equation C.15 is derived with respect to $x$ to obtain the equation of motion in terms of strain $\epsilon$.

$$
\rho_{0} \frac{\partial^{3} u}{\partial t^{2} \partial x}=\rho_{0} \frac{\partial^{2} \epsilon}{\partial t^{2}}=-\frac{\partial^{2} p}{\partial x^{2}}-\frac{\partial \tau_{w}}{\partial x} \frac{2}{r_{0}}
$$

The wall shear stress can be expressed as $\tau_{w}=\frac{4 \mu \frac{\partial u}{\partial t}}{r_{0}}$ as found for the fully developed laminar Hagen-Poiseuille flow in a circular tube. Since the process is also assumed to be isothermal, the temperature stays constant which means that the viscosity $\mu$ stays constant.

$$
\rho_{0} \frac{\partial^{2} \epsilon}{\partial t^{2}}=-\frac{\partial^{2} p}{\partial x^{2}}-\frac{\partial^{2} u}{\partial x \partial t} \frac{8 \mu}{r_{0}^{2}}=-\frac{\partial^{2} p}{\partial x^{2}}-\frac{\partial \epsilon}{\partial t} \frac{8 \mu}{r_{0}^{2}}
$$


The material law from equation C.16 is then inserted into equation C.27 to replace the expression for the pressure $p$. The derivatives of equation C.16 are

$$
\begin{aligned}
\frac{\partial p}{\partial x} & =-\frac{p_{0}}{(1+\epsilon)^{2}} \frac{\partial \epsilon}{\partial x} \\
\frac{\partial^{2} p}{\partial x^{2}} & =\frac{p_{0}}{(1+\epsilon)^{3}}\left(\frac{\partial \epsilon}{\partial x}\right)^{2}-\frac{p_{0}}{(1+\epsilon)^{2}} \frac{\partial^{2} \epsilon}{\partial x^{2}}
\end{aligned}
$$

The strain is assumed to be small $(\epsilon<<1)$ meaning that terms of $O\left(\epsilon^{2}\right)$ are neglected. The Taylor series of the fractions containing $\frac{1}{1+\epsilon}$ are

$$
\begin{aligned}
\frac{1}{1+\epsilon} & \approx f(\epsilon=0)+\frac{\partial f}{\partial x} \epsilon+O\left(\epsilon^{2}\right)=1-\epsilon+O\left(\epsilon^{2}\right) \\
\frac{1}{(1+\epsilon)^{2}} & \approx 1-2 \epsilon+O\left(\epsilon^{2}\right) \\
\frac{1}{(1+\epsilon)^{3}} & \approx 1-3 \epsilon+O\left(\epsilon^{2}\right)
\end{aligned}
$$

With these Taylor series approximations and the assumption that $\frac{\partial \epsilon}{\partial x}$ is at least of order $O(\epsilon)$, the material law and its derivatives connecting strain and pressure is

$$
\begin{aligned}
\frac{\partial p}{\partial x} & \approx-p_{0} \frac{\partial \epsilon}{\partial x} \\
\frac{\partial^{2} p}{\partial x^{2}} & \approx-p_{0} \frac{\partial^{2} \epsilon}{\partial x^{2}}
\end{aligned}
$$

In combination with equation C.27 the damped wave equation of the following form is obtained

$$
\frac{\partial^{2} \epsilon}{\partial t^{2}}+\frac{8 \mu}{\rho_{0} r_{0}^{2}} \frac{\partial \epsilon}{\partial t}=R T_{0} \frac{\partial^{2} \epsilon}{\partial x^{2}}
$$

The boundary conditions of this problem can be chosen as $\epsilon(0, t)=0$ and $\left.\frac{\partial \epsilon}{\partial x}\right|_{x=l}=$ 0 which corresponds to a closed end at $x=0$ and an open end at $x=l$. Eventually 
a source term of the form $\epsilon(x, t)=1-\frac{1}{p_{0}+p f \sin \left(\omega_{f} t\right)}$ for $x=l$ and $\epsilon(x, t)=0$ for $0 \leq x<l$ has to be added to equation C.27 to calculate the transfer function of the strain. With the source term, the solution of the homogeneous wave equation is superpositioned with the specific solution of the wave equation.

\section{C.1.4 Pitot Probe Conclusions}

Although the initially discussed models needed a lot of assumptions and simplifications, they show the upper limit of the expected bandwidth for an optimized high frequency Pitot tube measurement system. The actual frequency response and transfer function needs to be experimentally measured. Nonetheless, the conclusions which can be drawn from the two initially shown models are as follows. The diameter of the Pitot probe needs to be as large as possible, whereas the volume of the transducer cavity and especially length of the Pitot probe need to be minimized in order to increase the bandwidth. A very important factor to minimize the loss of bandwidth at smaller pressure ratios is the usage of a pressure transducer with negligible displacement of the internal volume when exposed to the pressure change. One method of achieving this is the integration of a pressure transducer inside the mounting wedge of the Pitot tube. In ideal case, the Pitot tube is directly attached to the pressure transducer. Small pressure transducers with frequency responses of several hundred $\mathrm{kHz}$ are manufactured e.g. by Kulite ${ }^{2}$. They are available in cylindrical shape down to a diameter of $1.7 \mathrm{~mm}$ and would fit inside the Pitot tube wedge. It seems that a bandwidth of several $\mathrm{kHz}$ can be achieved with the recommended Pitot probe dimensions for minimized probe interference. This could give some additional information

\footnotetext{
${ }^{2}$ Kulite Semiconductor Products, Inc., Leonia, NJ
} 
about the growth of disturbances in the boundary layer. Especially in case of the unexpected increase of overall pressure on the leeward side of the cone with plasma actuation, dynamic pressure measurements in the range of several $\mathrm{kHz}$ could have helped to understand better what happened to the boundary layer. Also some information could be obtained about the onset of transition in terms of spectrum broadening. Nonetheless, the very optimistically calculated response times of a dynamically optimized Pitot probe are still too low to either measure T-S waves which are expected to occur with tens of $\mathrm{kHz}$ or travelling cross flow modes which are most unstable in the range of 50 to $60 \mathrm{kHz}$ for our experiment. If secondary instabilities of the stationary cross flow instability could be detected, another distinct criterion for the location of the onset of turbulence can be found. These secondary instabilities occur at even higher frequencies which are about a factor of ten larger than the travelling cross flow modes. It is therefore important to improve the frequency response of the Pitot probe to shorten the waiting time in between two mean pressure measurements but most likely not for the purpose of taking meaningful dynamic data in supersonic flows. Anyhow, a dynamic Pitot tube sensor might be useful for low speed applications where the unstable frequencies are within the bandwidth of the Pitot probe. If high frequency dynamic data as in supersonic flows has to be taken with a pressure sensor, the diaphragm of the transducer itself has to be directly exposed to the flow for sufficient frequency response. This might be feasible e.g. in applications as static surface pressure sensor or for total pressure measurements in very thick boundary layers or free stream disturbance measurements. 


\section{C.2 Hot Wire Measurements}

It is thus necessary to use hot wire measurements if meaningful dynamic data in supersonic flow has to be taken. Hot wire measurements in the NASA Mach 3.5 SLDT were done by Matlis [34] and Kegerise [26]. Matlis designed a hot wire consisting of two 0.01 in stainless steel needles with $12 \mathrm{~mm}$ length and $1.5 \mathrm{~mm}$ spacing. The needles were glued with epoxy into a groove under the same steel wedge as used for the Pitot probe in the current work. The hot wire itself is Platinum plated Tungsten wire with $3.81 \mu \mathrm{m}$ diameter and manually soldered onto the tips of the needles. Special care was taken to ensure that the wire is not taut between the needle tips but instead with a bow so that vibrations of the needles during tunnel startup and shutdown do not break the hot wire. Matlis developed a method of in-situ calibration of the hot wire making it possible to calibrate the hot wire while running boundary layer measurements. He also used a hot wire to measure the uncalibrated mass flow fluctuations the Mach 3.5 cone boundary layer in combination with oblique 1st mode forcing with a cavity glow discharge actuator array. The ionized air of the plasma actuator caused the constant current anemometry circuit to overheat the wire which burned it. Therefore the hot wire electronics was designed with a battery driven power source to be able to run with floating ground. Frequency responses up to $500 \mathrm{kHz}$ could be achieved with Kegerise's constant temperature hot wire arrangement. Hot wire measurements, calibrated or uncalibrated, might be interesting for future work because information about eventually occurring secondary modes as well as intermittency or spectrum broadening can give additional information about the onset of laminar-turbulent transition. 


\section{APPENDIX D}

\section{DBD THRUST MEASUREMENTS}

\section{D.1 Experimental DBD Plasma Body Force Study in Sub-Atmospheric Pressure}

Plasma actuators as active flow control devices in the context of laboratory experiments are usually used at atmospheric pressure in air. However, a lot of environments in which the target application of a plasma actuator lies, will be at non-atmospheric pressures. One example is the usage of plasma actuators in compressor stages of a turbine on the high pressure side or the usage of a plasma actuator in low pressure environments like high altitude flight. The experiments described in this thesis are of course also part of the low pressure experimentation. In order to empirically investigate the effects of sub-atmospheric pressure on the performance of the plasma actuator, thrust measurements in a vacuum vessel at

different pressures are done. The results contribute to the understanding of plasma actuators and deliver further input for the numerical modeling of DBD plasma actuators.

The independent variable of the experiments is body force or measured thrust force. Dependent variables are ambient pressure, AC voltage and the amplitude of the input voltage. 


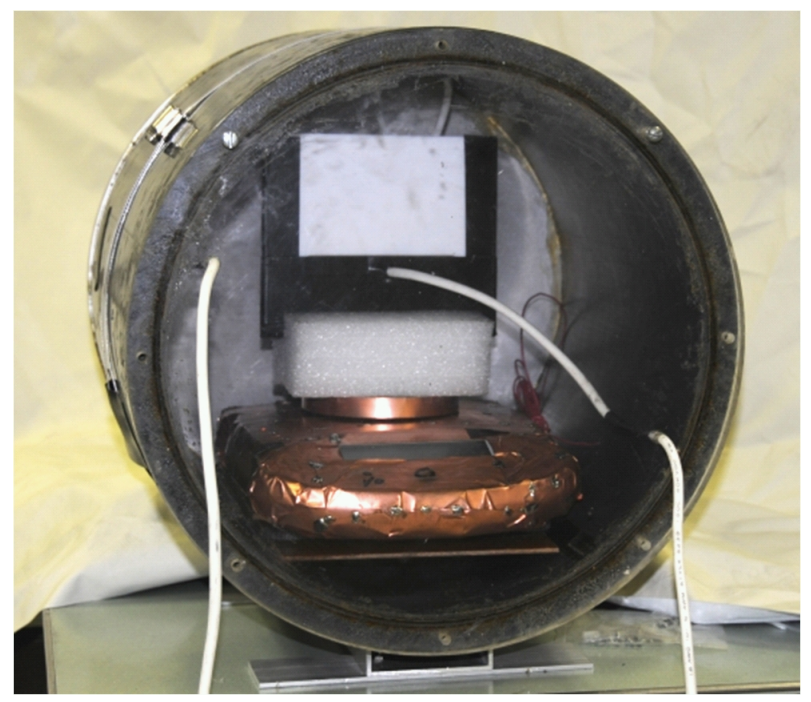

Figure D.1. Teflon plasma actuator on the shielded force balance inside the vacuum vessel.

\section{D.1.1 Experimental Setup}

A standard straight edge DBD plasma actuator was mounted vertically on the weighing pan of a Acculab ALC 320.3 scale with a total range of $320 \mathrm{~g}$ and a repeatability of $1 \mathrm{mg}$. The generated tangential wall jet points away from the scale which means that the plasma actuator pushes on the surface of the force balance. The scale itself was covered with copper tape and electrically grounded to minimize electromagnetic interference between the high voltage wire of the plasma actuator and the scale. The entire setup was situated in a vacuum vessel made of a PVC tube and two endplates. A picture of the entire experimental setup is shown in figure D.1. 


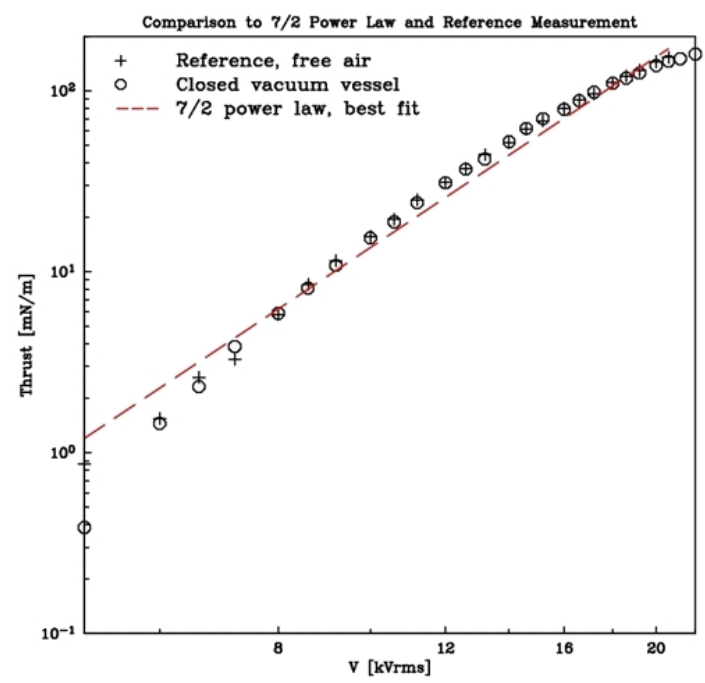

Figure D.2. Comparison of thrust measurements inside and outside the vacuum vessel.

\section{D.1.2 Actuator Measurements}

Several dielectrics with different thicknesses and electrode widths and spans were used for this study. The waveforms used were ramps and all thrust values are normalized with the width of the electrodes. The first actuator consists of $1 / 4$ in thick Quartz glass with an overlap of $1 / 8$ in and a span of only $2.5 \mathrm{in}$. The width of the covered electrode is $1 \mathrm{in}$. The first measurement was a comparison between the measured thrust inside the vacuum vessel and outside the vacuum vessel. Figure D.2 shows the obtained curves including a fit to the $7 / 2$ power law. It can be seen that there is no difference in between the measurements and that the data points match the power law relatively well. In the next step, the $\mathrm{AC}$ driving frequency was kept constant at $2 \mathrm{kHz}$ ramps and the influence of ambient pressure on the thrust was obtained. The ambient pressure inside the vacuum vessel was reduced in several steps down to about $8 \mathrm{inHg}$. For each pressure, the 


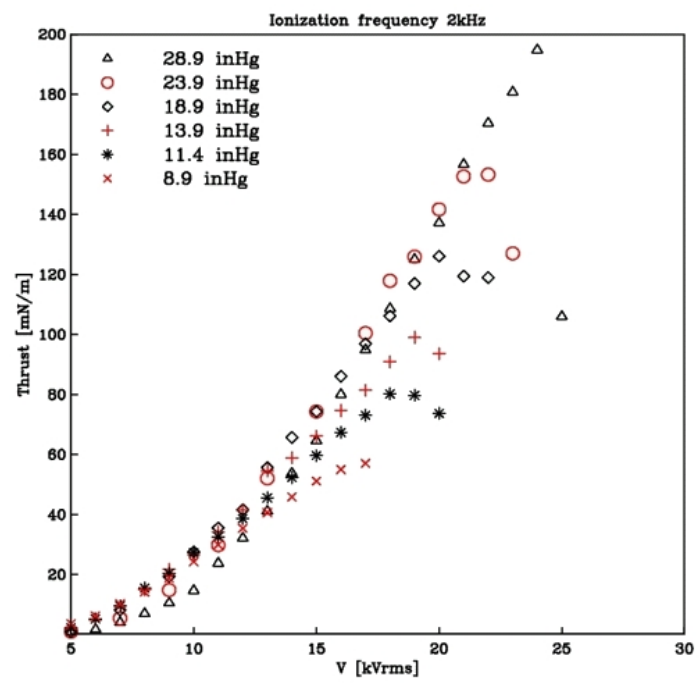

Figure D.3. Influence of different ambient pressures on the voltage vs. thrust curves. $2 \mathrm{kHz}$ ramps.

input voltage versus thrust curve was recorded as shown in figure D.3. The voltages at which the thrust reaches its maximum is coincidental with the formation of filamentary discharges.

A very similar behavior can be seen when the ambient pressure is kept constant at atmospheric pressure and the $\mathrm{AC}$ driving frequency of the plasma actuator is increased. The thrust increases up to a certain point and falls off quickly. The maximum thrust is reached whenever the plasma discharge shows strong filamentary discharges over the region of the covered electrode. Figure D.4 shows the measured curves.

Based on repeated measurements with different $\mathrm{AC}$ driving frequencies and different pressures, a figure of maximum thrust in dependence of ambient pressure and frequency is compiled. The result can be seen in figure D.5. The maximum pressure is obtained with the lowest $\mathrm{AC}$ driving frequency for all tested ambient 


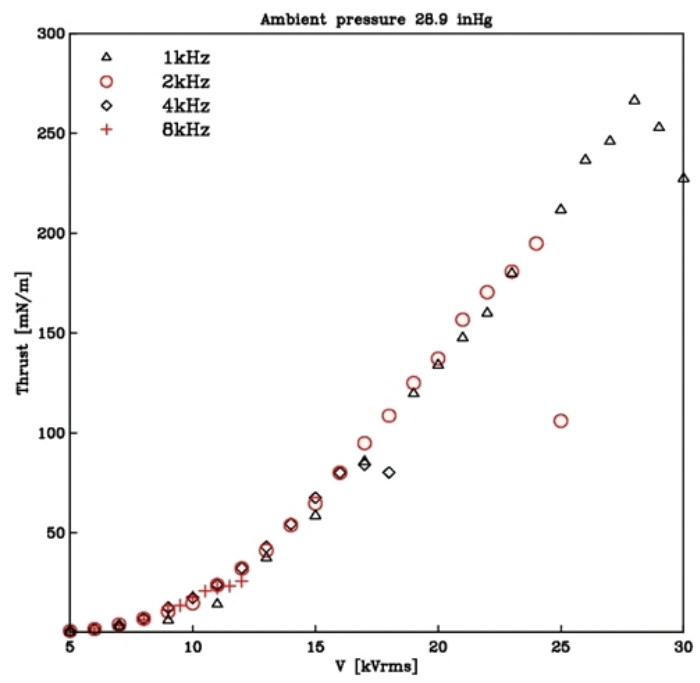

Figure D.4. Influence of different ambient pressures on the voltage vs. thrust curves. $2 \mathrm{kHz}$ ramps.

pressures and decreases with decreasing ambient pressure. Because the thrust is limited by the maximum voltage at which the filamentary discharge occurs, filamentary discharges seem to appear earlier at lower voltages with higher frequencies and lower ambient pressures.

Another interesting question is, how the thrust develops for the remaining part of the voltages, far below the voltage at which filamentary discharges occur. For that purpose, figure D.6 shows the development of thrust in dependence of the ambient pressure when the amplitude of the applied voltage is kept constant. The interesting observation is, that the thrust increases at when the ambient pressure is reduced and the applied voltage is kept constant. The values shown are just for lower voltages since the data points will reach the filamentary regime earlier towards the lower pressures. This will diminish the just shown effect.

The same effect can be shown when the voltage needed to sustain a certain 


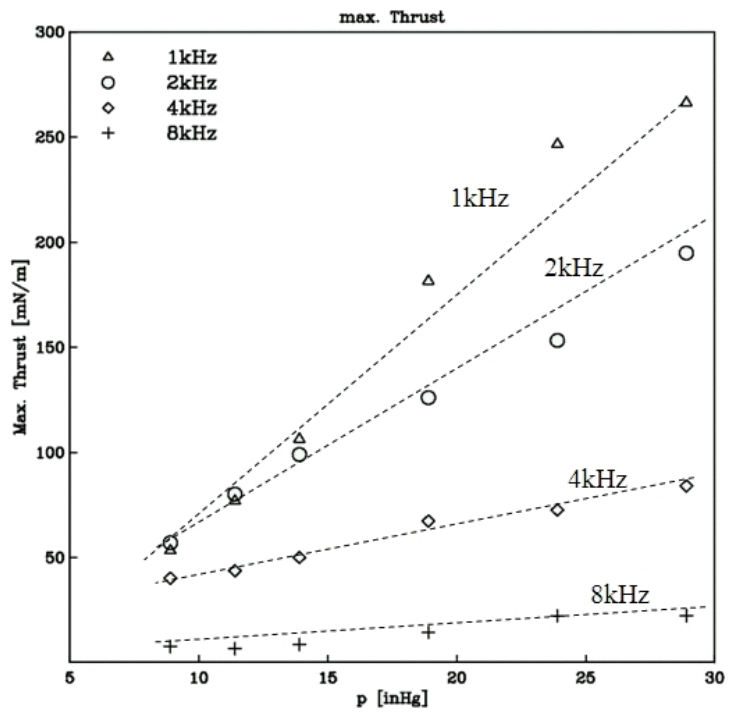

Figure D.5. Maximum thrust values in dependence of ambient pressure for different $\mathrm{AC}$ driving frequencies.

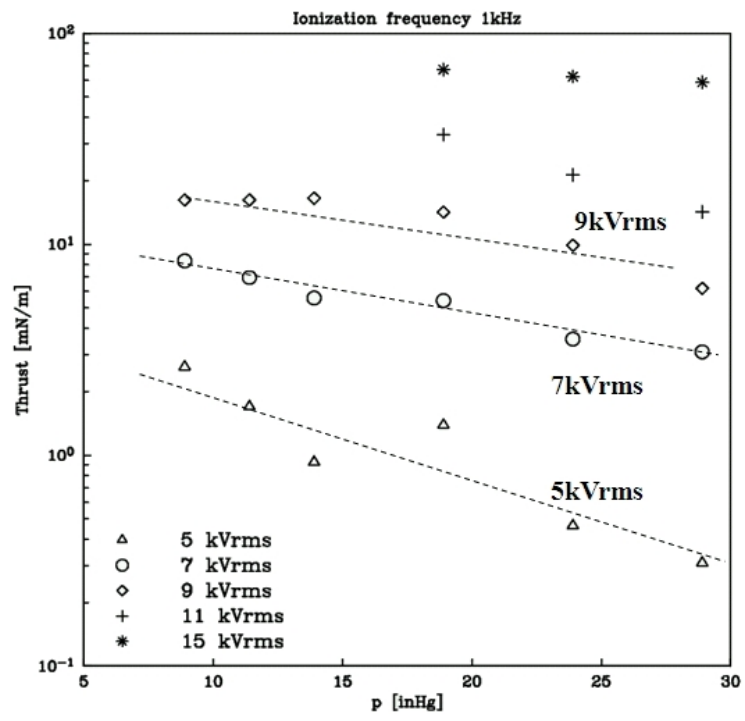

Figure D.6. Dependence of thrust at constant applied voltage on ambient pressure. 


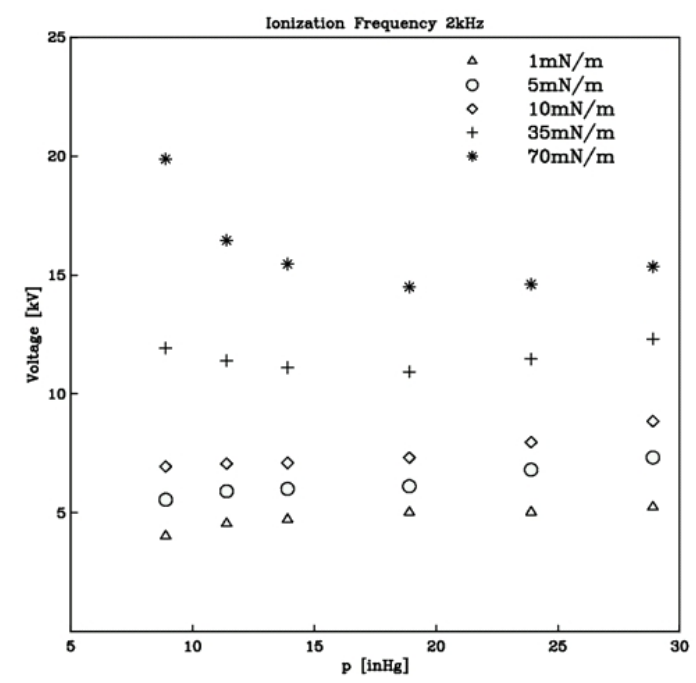

Figure D.7. Necessary applied voltage to maintain constant body force for different ambient pressures.

amount of thrust is plotted versus the ambient pressure. For low values of thrust, the voltage needed to maintain those values goes down with decreasing pressure. If a larger thrust value is chosen, the discharge gets closer to the filamentary regime with decreasing pressure and the voltage necessary to maintain that level of thrust increases.

The final question to be answered is how the AC driving frequency affects the amount of thrust produced with decreasing ambient pressure. For that purpose, figure $\mathrm{D} .8$ shows again the amount of thrust which is produced by a constant applied voltage at different ambient pressures. This time, the data for 1,2,4, and $8 \mathrm{kHz} \mathrm{AC}$ driving frequency is shown. As expected, the thrust increases when increasing the $\mathrm{AC}$ driving frequency for all shown applied voltage amplitudes in the far pre-filamentary regime. The most notable increase in thrust happens from 1 to $2 \mathrm{kHz}$. The thrust values for higher frequencies are less distinguishable which 


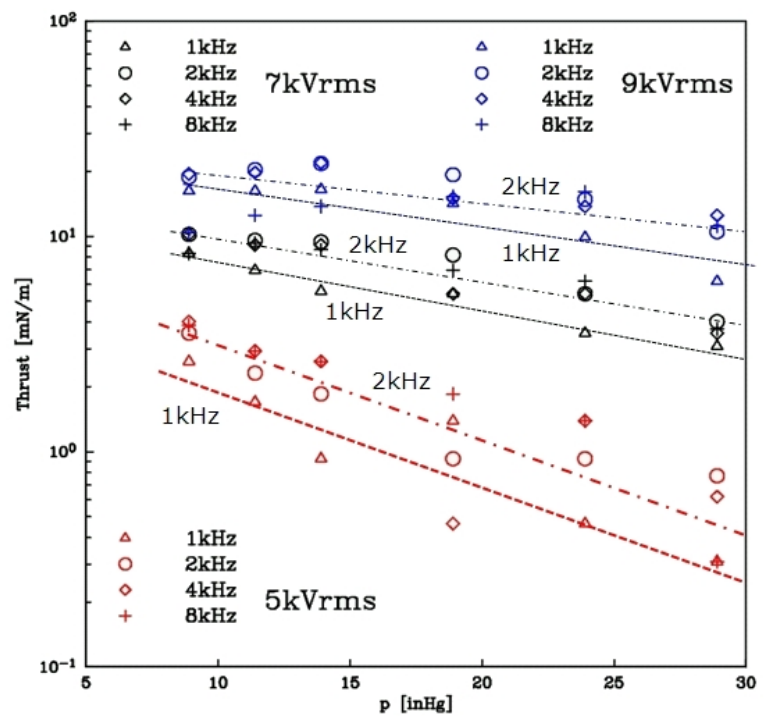

Figure D.8. Necessary applied voltage to maintain constant body force for different ambient pressures.

might also be a consequence of the logarithmic representation of the data.

There are several trends which can be derived from the data.

1. The maximum thrust is limited by the formation of filamentary discharges.

2. Filamentary discharges happen at lower applied voltage amplitudes for higher $\mathrm{AC}$ driving frequencies and lower ambient pressures.

3. The same constant applied voltage amplitude generates more thrust when the ambient pressure is reduced, as long as the voltage amplitudes are in the pre filamentary regime.

4. Increasing the $\mathrm{AC}$ driving frequency increases the body force over the entire range of tested pressures.

Finally, the effect of the width of the covered electrode for different AC driving frequencies and ambient pressures is investigated. Two different actuators were 

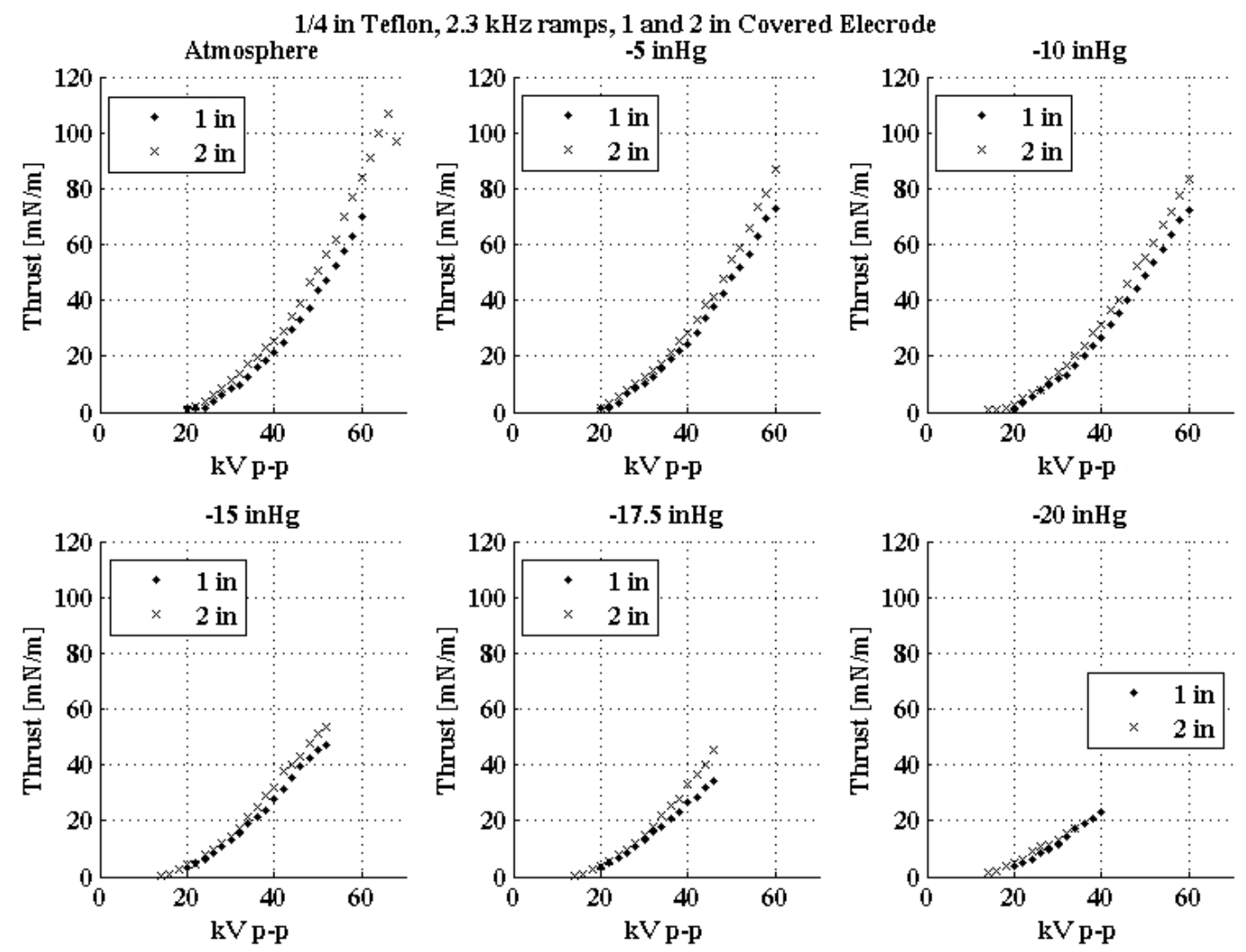

Figure D.9. Thrust measurements for a $1 / 4$ in thick Teflon dielectric with 1 in and 2 in wide covered electrodes at different ambient pressures.

used for this purpose. The actuator to investigate the difference between two different covered electrode widths at different ambient pressures consists of a $1 / 4$ in thick Teflon dielectric with 2.75 in span and 1 in and 2 in wide covered electrodes. The results are shown in figure $\mathrm{D} .9$ for $2.3 \mathrm{kHz}$ ramps. It can be seen that the thrust for all ranges of pressure is smaller with the 1 in wide covered electrode compared to the 2 in wide covered electrode. The relative difference between the two electrode widths stays almost the same over the entire tested pressure range.

The corresponding thrust measurements at constant atmospheric pressure but changing AC driving frequency are shown in figure D.10. A very similar effect can 

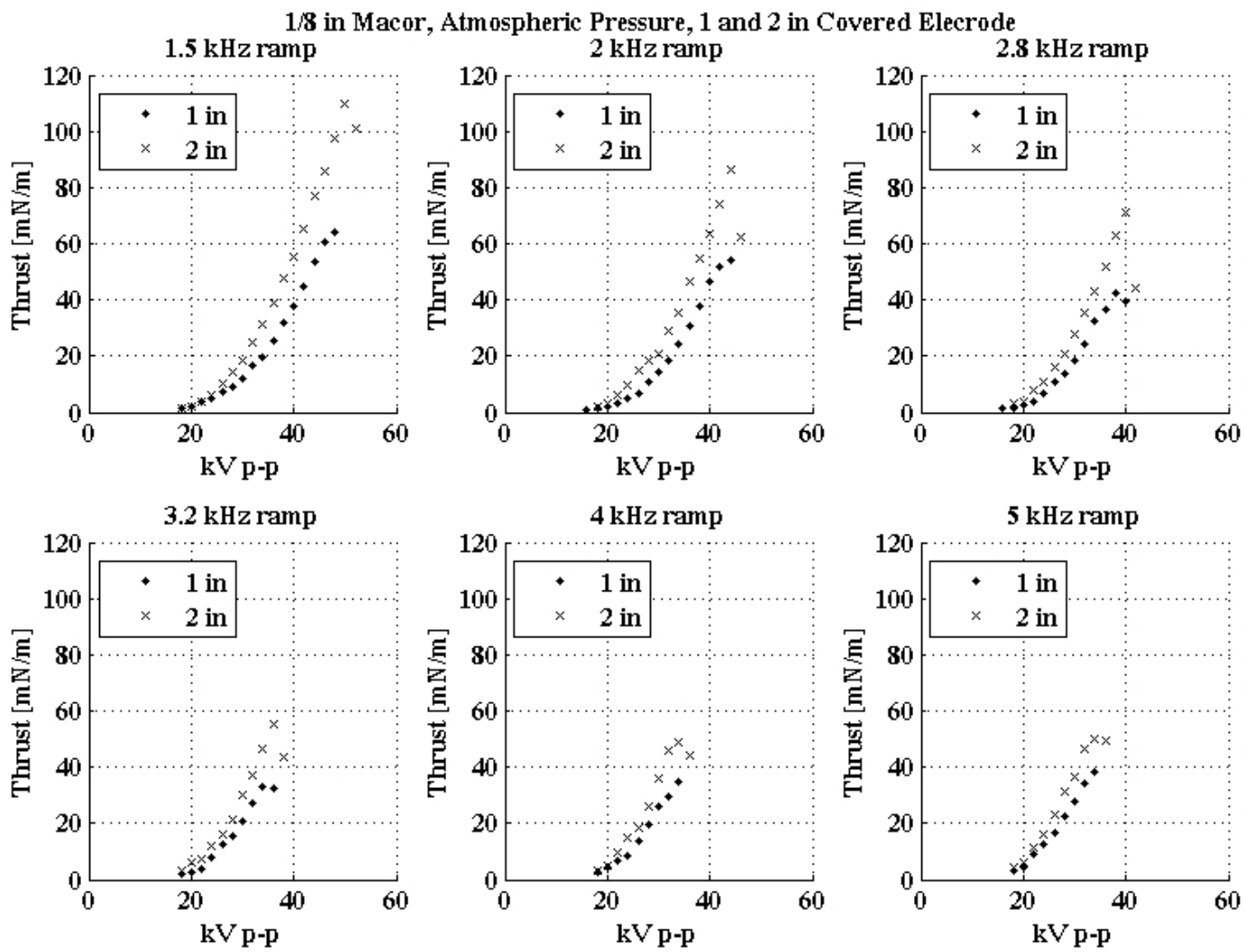

Figure D.10. Thrust measurements for a $1 / 8$ in thick Macor dielectric with 1 in and 2 in wide covered electrodes at constant pressures and different $\mathrm{AC}$ driving frequencies.

be seen which shows that the smaller electrode width leads to lower thrust values relative to the 2 in width covered electrode. 


\section{BIBLIOGRAPHY}

[1] C. Airiau, S. Walther, and A. Bottaro. Boundary Layer Sensitivity and Receptivity. C.R. Meccanique, 330:259-265, 2002.

[2] J.D. Anderson Jr. Fundamentals of Aerodynamics. McGraw-Hill, Inc., 1991.

[3] P. Balakumar. Stability of Supersonic Boundary Layers on a Cone at an Angle of Attack. 39th Fluid Dynamics Conference and Exhibit, June 22-25,2009, San Antonio, Texas AIAA-2009-3555, 2009.

[4] Ivan E. Beckwith and Mitchel H. Bertram. A Survey of NASA Langley Studies on High-Speed Transition and the Quiet Tunnel. NASA-TM X-2566, 1972.

[5] H. Bippes. Basic Experiments on Transition in Three-dimensional Bboundary Layers dominated by crossflow instability. Progress in Aerospace Sciences, 35:363-412, 1999.

[6] P. Bletzinger, B. N. Ganguly, D. Van Wie, and A. Garscadden. PLasmas in High Speed Aerodynamics. Journal of Physics D: Applied Physics, 38:R33R57, 2005.

[7] A. Busemann. Aerodynamischer Auftrieb bei Ueberschallgeschwindigkeit. Luftfahrtforschung, 12:210-215, 1935. 
[8] D. Bushnell. Research Status/Requirements and NASA Applications for Wall Layer Transition. Proceedings of the Workshop on Transition, Turbulence and Combustion, Volume I, Transition, 1993.

[9] D. Cavalieri. On the Experimental Design for Instability Analysis on a Cone at Mach 3.5 and 6 using Corona Discharge Pertubation Method. M.S. Thesis, Illinois Institute of Technology, Chicago, IL, 1995.

[10] Valery G. Chernoray, Alexander V. Dovgal, Victor V. Kozlov, and Lennart Loefdahl. Experiments on Secondary Instability of Streamwise Vortices in a Swept-Wing Boundary Layer. Journal of FLuid Mechanics, 534:295-325, 2005.

[11] P. Coen, M.J. Long-Davis, and L. Provinelli. Fundamental Aeronautics Program Supersonic Project Reference Document. Website, http://www. aeronautics.nasa.gov/nra_pdf/sup_proposal_c1.pdf.

[12] T. Corke, F. Thomas, D. Schatzman, and T. Wood. United States Patent Application US 20110120980A1, May 26, 2011.

[13] T. C. Corke, C. L. Enloe, and S. P. Wilkinson. Dielectric Barrier Discharge Plasma Actuators for Flow Control. Annual Review of Fluid Mechanics, 42:505-529, 2010.

[14] J.R. Dagenhart, W.S. Saric, M.C. Mousseaux, and J.P. Stack. Crossflow Vortex Instability and Transition on a 45-Degree Swept Wing. AIAA 891892, 1989.

[15] H. Deyhle and H. Bippes. Disturbance Growth in an Unstable Three- 
Dimensional Boundary Layer and its Dependence on Environmental Conditions. Journal of Fluid Mechanics, 316:73-113, 1996.

[16] V. DiCrisitina. Three-Dimensional Laminar Boundary-Layer Transition on a Sharp $8^{\circ}$ Cone at Mach 10. AIAA Journal, 8(5):852-856, 1970.

[17] P. F. Dunn. Measurement and Data Analysis for Engineers and Science. McGraw-Hill series in mechanical engineering, 2005.

[18] David F. Fisher and N.Sam Dougherty Jr. In-Flight Transition on a $10^{\circ}$ Cone at Mach Numbers from 0.5 to 2. NASA-TP-1971, 1982.

[19] Northrop Grumman. Quiet Supersonic Platform (QSP), Shaped Sonic Boom Demonstrator (SSBD) Program. Website, 2003. http://www.faa.gov/about/office_org/headquarters_offices/AEP/ supersonic_noise/media/3-Panel1-Henne_Gulfstream.pdf

[20] S. Grundmann and C. Tropea. Experimental Damping of Boundary-Layer Oscillations using DBD Plasma Actuators. International Journal of Heat and Fluid Flow, 30:394-402, 2009.

[21] R.A.W.M. Henkes and J.L. Ingen, editors. Transitional Boundary Layers in Aeronautics. Royal Netherlands Academy of Arts and Sciences, December 1996. T. Lerche and H. Bippes: Experimental Investigation of Cross-Flow Instability Under the Influence of Controlled Disturbance Excitation, ISBN 0-444-85812-1.

[22] Preston A. Henne. A Gulfstream Perspective on the DARPA QSP Program and Future Civil Supersonic Initiatives. Website, 
2003. http://www.faa.gov/about/office_org/headquarters_offices/ AEP/supersonic_noise/media/2-Panel1-Graham-Northrop.pdf.

[23] Donald C. Howe and Kenrick A. Waithe. Quiet Spike Near Field Flight Test Pressure Measurements with Computational Fluids Dynamics Comparisons. 46th AIAA Aerospace Sciences Meeting and Exhibit AIAA 2008-128, January 2008 .

[24] L.N. Cattafesta III, V. Iyer, J.A. Masad, R.A. King, and J.R. Dagenhart. Three-Dimensional Boundary-Layer Transition on a Swept Wing at Mach 3.5. AIAA Journal, 33(11):2032-2037, 1995.

[25] Yu.S. Kachanov. Three-Dimensional Receptivity of Boundary Layers. Eur. J. Mech. B-Fluids, 19:723-744, 1999.

[26] M. A. Kegerise, L. R. Owens, and R. A. King. High-Speed Boundary-Layer Transition induced by an Isolated Roughness Element. 40th Fluid Dynamics Conference and Exhibit, 28 June - 1 July, Chicago, Illinois.

[27] E. H. Kennard. Kinetic Theory of Gases. McGraw-Hill Book Company, Inc., 1938.

[28] R.A. King. Three-Dimensional Boundary-Layer Transition on a Cone at Mach 3.5. Experiments in Fluids, 13:305-314, 1992.

[29] R. Kobayashi, Y. Kohama, and M. Kurosawa. Boundary-layer Transition on a Rotating Cone in Axial Flow. Journal of Fluid Mechanics, 127:341-352, 1983.

[30] S. B. Leonov and D. A. Yarantsev. Near-Surface Electrical Discharge in 
Supersonic Airflow: Poperties and Flow Control. Journal of Propulsion and Power, 24:1168-1181, 2008.

[31] Fei Li, Meelan Choudhari, Mark Carpenter, Mujeeb Malik, Chau-Lyan Chang, and Craig Streett. Roughness Based Crossflow Transition Control for a Swept Airfoil Design Relevant to Subsonic Transports . 28th AIAA Applied Aerodynamics Conference 28 June - 1 July 2010, Chicago, Illinois, 2010.

[32] Fei Li, Meelan Choudhari, Chau-Lyan Chang, Craig Streett, and Mark Carpenter. Roughness Based Crossflow Transition Control: A Computational Assessment. 27th AIAA Applied Aerodynamics Conference 22 - 25 June 2009, San Antonio, Texas, 2009.

[33] G. G. Mateer. The Effect of Angle of Attack on Boundary-Layer Transition on Cones. AIAA Journal, 10(8):1127-1128, 1972.

[34] Eric H. Matlis. Controlled Experiments on Instabilities and Transition to Turbulence on a Sharp Cone at Mach 3.5. PhD Thesis, University of Notre Dame, Notre Dame, IN, 2003.

[35] E. Moreau. Airflow Control by Non-Thermal Plasma Actuators. Journal of Physics D: Applied Physics, 40:605-636, 2007.

[36] M.N. Nishihara, K. Takashima, J.W. Rich, and I.V. Adamovich. Mach 5 Bow Shock Control by a Nanosecond Pulse Surface Dielectric Barrier Discharge. Physics of Fluids, 23, 2011.

[37] I. Poll. Some Observations of the Transition Process on the Windward Face of a Long Yawed Cylinder. Journal of Fluid Mechanics, 150:329, 1985. 
[38] R.H. Radeztsky Jr., M.S. Reibert, and W.S. Saric. Effect of Isolated Micron-Sized Roughness on Transition in Swept-Wing Flows. AIAA Journal, 37(11):1370-1377, 1999.

[39] H. L. Reed and W. S. Saric. Stability of Three-Dimensional Boundary Layers. Annual Review of Fluid Mechanics, 21:235-284, 1989.

[40] D.V. Roupassov, A.A. Nikipelov, M.M. Nudnova, and A. Yu. Starikovskii. Flow Seprataion Control by Plasma Actuator with Nanosecond PulsePeriodic Discharge. AIAA Journal, 47:168-185, 2009.

[41] A. Santhanakrishnan and J. D. Jacob. Flow Control Using Plasma Actuators and Linear/Annular Plasma Synthetic Jet Actuators. 3rd AIAA Flow Control Conference, San Francisco, CA, June 5-8, San Francisco, CA.

[42] W. S. Saric. Private Communication, 2008.

[43] W. S. Saric and H. L. Reed. Supersonic Laminar Flow Control on Swept Wings Using Distributed Roughness. AIAA Paper AIAA 2002-0147, 2002.

[44] William S. Saric, Carillo Ruben C, and Mark S. Reibert. Leading-Edge Roughness as a Transition Control Mechanism. AIAA Paper AIAA-98-0781, 1998.

[45] W.S. Saric, R.B. Carrillo, and M.S. Reibert. Nonlinear Stability and Transition in 3-D Boundary Layers. Meccanica, 33:469-187, 1998.

[46] W.S. Saric and H. Reed. Control of Stability and Transition in SweptWing Boundary Layers. Website. http://flight.tamu.edu/pubs/ presentations/swept-wing.pdf. 
[47] W.S. Saric and H.L. Reed. Crossflow Instabilities-Theory \& Technology. AIAA Paper 2003-0771.

[48] W.S. Saric, H.L. Reed, and D.W. Banks. Flight Testing of Laminar Flow Control in High-Speed Boundary Layers. Paper presented at the RTO AVT Specialists' Meeting on 'Enhancement of NATO Military Flight Vehicle Performance by Managemenet of Interacting Boundary Layer Transition and Separation', held in Prague, Czech Republic, 4-7 October 2004, Published in RTO-MP-AVT-111, 2004.

[49] W.S. Saric, H.L. Reed, and E.B. White. Stability and Transition of ThreeDimensional Boundary Layers. Annual Review of Fluid Mechanics, 35:413440, 2003.

[50] Corning SAS. Macor. Website. http://www.corning.com/WorkArea/ showcontent . aspx?id=40321.

[51] H. Schlichting. Boundary Layer Theory. McGraw-Hill Book Company, Inc., 1960.

[52] N.V. Semionov, Yu.G. Ermolaev, and A.D. Kosinov. Evolution of Disturbances in a Laminarized Supersonic Boundary Layer on a Swept Wing. Journal of Applied and Technical Physics, 49(2):188-193, 2008.

[53] N.V. Semionov, A.D. Kosinov, and V.Ya. Levchenko. Experimental Study of Turbulence Beginning and Transition Control in a Supersonic Boundary Layer on Swept Wing. R. Govindarajan (ed.), Sixth IUTAM Symposium on Laminar Turbulent Transition, 2006. 
[54] A. R. Sinclair and A. W. Robins. A Method for the Determination of the Time Lag in Pressure Measuring Systems Incorporating Capillaries. NASA TM 2793, 1952.

[55] P. Calvin Stainback. Effect of Uint Reynolds Number, Nose Bluntness, Angle of Attack, and Roughness on Transition on a $5^{\circ}$ Half-Angle Cone at Mach 8. NASA-TN D-4961, 1969.

[56] K. F. Stetson. Hypersonic Boundary Layer Transition Experiments. AFWALTR-80-3062, 1980.

[57] Kenneth F. Stetson and George H. Rushton. Shock Tunnel Investigation of Boundary-Layer Transition at M=5.5. AIAA Journal, 5:899-906, 1967.

[58] K. F. Knasiak T. C. Corke. Stationary Travelling Cross-Flow Mode Interactions on a Rotating Disk. Journal of Fluid Mechanics, 355:285-315, 1998.

[59] J. Valerioti. Pressure Dependence of Plasma Actuated Flow Control. MS Thesis, University of Notre Dame, Notre Dame, IN, 2010.

[60] E. B. White and W. S. Saric. Secondary Instability of Crossflow Vortices. Journal of Fluid Mechanics, 525:275-308, 2005.

[61] F. M. White. Viscous Fluid Flow. McGraw-Hill, 1221 Avenue of the Americas, New York, NY 10020, 2006.

[62] S. P. Wilkinson. Private Communication, 2011.

[63] S. P. Wilkinson and M. R. Malik. Stability Experiments in the FLow over a Rotating Disk. AIAA Journal, 23(4):588-595, 1998. 
[64] S.P. Wilkinson, S.G. Anders, F.J. Chen, and I.E. Beckwith. Supersonic and Hypersonic Quiet Tunnel Technology at NASA Langley. AIAA 17 Aerospace Ground Testing Conference, Nashville, TN, July 6-8, 1992.

[65] Stephen W.D. Wolf and James A. Laub. NASA Ames Laminar Flow Supersonic WInd Tunnel (LFSWT) Tests of a $10^{\circ}$ COne at Mach 1.6. NASA-TM 110438, 1997.

[66] M. J. Zucrow and J. D. Hoffman. Gas Dynamics, volume 1. John Wiley and Sons, 1976. 
This document was prepared 85 typeset with $\mathrm{pdfIAT} \mathrm{E}$, and formatted with $\operatorname{NDdiss}_{\varepsilon} \varepsilon$ classfile (v3.0[2005/07/27]) provided by Sameer Vijay. 